\title{
Interventional Oncology in the Management of Metastatic Colorectal Cancer
}

Citation for published version (APA):

Kurilova, I. (2020). Interventional Oncology in the Management of Metastatic Colorectal Cancer. [Doctoral Thesis, Maastricht University]. Gildeprint en Universitaire Pers Maastricht. https://doi.org/10.26481/dis.20200707ik

Document status and date:

Published: 01/01/2020

DOI:

10.26481/dis.20200707ik

Document Version:

Publisher's PDF, also known as Version of record

\section{Please check the document version of this publication:}

- A submitted manuscript is the version of the article upon submission and before peer-review. There can be important differences between the submitted version and the official published version of record.

People interested in the research are advised to contact the author for the final version of the publication, or visit the DOI to the publisher's website.

- The final author version and the galley proof are versions of the publication after peer review.

- The final published version features the final layout of the paper including the volume, issue and page numbers.

Link to publication

\footnotetext{
General rights rights.

- You may freely distribute the URL identifying the publication in the public portal. please follow below link for the End User Agreement:

www.umlib.nl/taverne-license

Take down policy

If you believe that this document breaches copyright please contact us at:

repository@maastrichtuniversity.nl

providing details and we will investigate your claim.
}

Copyright and moral rights for the publications made accessible in the public portal are retained by the authors and/or other copyright owners and it is a condition of accessing publications that users recognise and abide by the legal requirements associated with these

- Users may download and print one copy of any publication from the public portal for the purpose of private study or research.

- You may not further distribute the material or use it for any profit-making activity or commercial gain

If the publication is distributed under the terms of Article $25 \mathrm{fa}$ of the Dutch Copyright Act, indicated by the "Taverne" license above, 


\section{Interventional Oncology in the Management of Metastatic Colorectal Cancer}

Ieva Kurilova 


\title{
Interventional Oncology in the Management of Metastatic Colorectal Cancer
}

\author{
PROEFSCHRIFT
}

Ter verkrijging van de graad van doctor aan de Universiteit Maastricht, Op gezag van de Rector Magnificus, Prof. Dr. Rianne M. Letschert

Volgens het besluit van het College van Decanen,

In het openbaar te verdedigen

Op dinsdag 7 juli 2020 om 14.00 uur

ISBN:

Cover image:

Cover Lay-out:

Lay-out:

Printing:

978-94-6402-340-4

(C)2015, Memorial Sloan Kettering Cancer Center

lse Modder | www.ilsemodder.nl

Ilse Modder | www.ilsemodder.nl

Gildeprint Enschede | www.gildeprint.nl door

Ieva Kurilova

The publication of this thesis was financially supported by the Netherlands Cancer Institute and by the Maastricht University.

Copyright $\left({ }^{2} 2020\right.$ I. Kurilova.

All rights reserved. No part of this thesis may be reproduced, stored or transmitted in any form or by any means without the permission of the author. The copyright articles of the articles that have been published had been transferred to the respective publishers. 


\section{Supervisors:}

Prof. Dr. Regina G.H. Beets-Tan

Prof. Dr. Constantinos T. Sofocleous, Memorial Sloan Kettering Cancer Center, New York, USA

\section{Assessment committee:}

Prof. Dr. C.H.C. Dejong (chairman)

Dr. G.H. Koek

Prof. Dr. M.R. Meijerink, Amsterdam University Medical Centers (Amsterdam UMC),

Amsterdam, NL

Prof. Dr. F. M. Mottaghy

Prof. Dr. C. Verhoef, Erasmus MC Cancer Institute, Rotterdam, NL

\section{CONTENTS}

Chapter 1. General Introduction and Outline of the Thesis

PART 1.

Chapter 2.

Chapter 3 .

Chapter 4 .

Chapter 5 .

Chapter 6.

Chapter 7 .

Chapter 8.

PART 3.

Chapter 9 .

Chapter 10.

Chapter 11.

Chapter 12.

Chapter 13

Chapter 14
Management of colorectal liver metastases in

\section{interventional oncology setting}

Liver-Directed and Systemic Therapies for Colorectal Cancer Liver Metastases

Factors Associated with Local Tumor Control and

Complications after Thermal Ablation for Colorectal Cancer

Liver Metastases: a 15-years Retrospective Cohort Study

Factors Affecting Oncologic Outcomes of ${ }^{\circ} \mathrm{Y}$

Radioembolization of Heavily Pre-Treated Patients with

Colon Cancer Liver Metastases

${ }^{\circ} \mathrm{Y}$ Resin Microspheres Radioembolization for Colon Cancer Liver Metastases Using Full-Strength Contrast Material

9oY Radiation Segmentectomy of Hepatic Metastases:

Technical Considerations, Correlations to Dosimetry and

Preliminary Results

\section{Management of colorectal lung metastases in}

interventional oncology setting

Image-Guided Thermal Ablation for the Treatment of

Pulmonary Malignancies

Microwave Ablation in the Management of Colorectal

Cancer Pulmonary Metastases

General discussion, summary and valorization

General Discussion, Future Perspectives and Conclusion

Summary

Valorization

List of Publications

Acknowledgements

Curriculum Vitae
205

207

217

225

231

235 
General introduction and outline for the thesis 


\section{INTRODUCTION}

It is estimated that at least 1 in 25 people will be diagnosed with colorectal cancer (CRC) at some point during their lifetime [1]. In the past decades minimally invasive approaches have increasingly been adopted as part of the spectrum of treatment options for metastatic CRC. These therapies aim to achieve complete tumor eradication or local control of the metastatic lesions with limited morbidity.

Interventional oncology (IO) has established itself as a pillar in the armamentarium of minimal invasive therapies alongside with surgery and radiation therapy [2]. As a result, there is an international consensus, that interventional radiologists should be standing members of institutional CRC tumor boards and of dedicated guideline bodies such as the National Comprehensive Cancer Network [3]. There is evidence that the utilization of interventional therapies can increase the overall survival of CRC patients [4, 5].

IO has multiple advantages compared to other local and loco-regional therapies for metastatic CRC. Compared to surgical resection, IO procedures carry lower morbidity and mortality rates, less direct economic costs and shorter hospitalization stay [6-8]. Compared to radiation oncology, IO procedures are generally less expensive; do not require multiple treatment sessions or treatment effective dose modification to spare healthy tissues at risk in case of unfavorable tumor location [9]. While systemic therapies remain the mainstay in treatment of metastatic CRC, they very rarely result in complete tumor eradication or cure. IO techniques have potential to prolong patient's survival by eradicating total tumor load without systemic toxicity. IO treatments can be used as a single treatment or combined with systemic therapies to further improve disease-free and overall survival.

In CRC patients with oligometastatic disease IO treatment is specifically useful in those patients, who not amenable to resection and those at risk for subsequent recurrences and the need for additional local treatments. Oligometastatic disease is defined as small volume disease, localized to a few sites - usually up to 2-3 organs with up to 5 distinct tumors [10]. While small volume oligometastatic CRC is potentially curable and local control can be obtained with potentially curative interventional treatments such as tumor ablation [11], patients with more advanced metastatic disease and substantial liver-confined tumor load may also benefit better from liver directed, IO intra-arterial therapies.

Image-guided percutaneous procedures are available for different stages of metastatic disease (early, intermediate or advanced/chemorefractory) as well as in different treatment settings (monotherapy, neoadjuvant, adjuvant and salvage setting) [12-20].

Thermal ablation is the most common percutaneous image guided ablative technique for oligometastatic CRC [4,5,21-25]. Thermal ablation utilizes extreme hyperthermia ( $>60$ $\left.{ }^{\circ} \mathrm{C}\right)$ or hypothermia $\left(<-40^{\circ} \mathrm{C}\right)$ delivered via needles which directly target the tumor aiming to achieve complete tumor cell death [26]. There are several techniques available, radiofrequency (RFA) [27-32], microwave (MWA) [33-36], laser ablations [37-39], high intensity focused ultrasound (HIFU) [40] and cryoablation [41], with each technique having its specific indications. RFA and MWA are the most widely adopted techniques in metastatic CRC [42].

Radioembolization is a form of intra-arterial brachytherapy where tiny microspheres (20-6o $\mu \mathrm{m}$ in size) impregnated with radioactive isotope (most commonly yttrium-9o, ${ }^{\circ} \mathrm{Y}$ ) are permanently injected in the tumor-feeding blood vessels emitting high doses of radiation for the following two weeks. Radioembolization is mostly used in the advanced and chemorefractory stage of CRC metastatic disease. Several studies showed safety and efficiency of this therapy in controlling the liver disease with response rates usually over $40 \%$ (ranging from 10 to $55 \%$ ) [43], that are at the higher end of spectrum of response amongst therapeutic options for the patients with advanced and chemorefractory stage of disease.

Despite multiple advantages in the field of local and locoregional therapies the relatively high local tumor progression rates in general remain a significant problem for interventional therapies too. In routine practice the proper sequence of locoregional therapies pose a daily challenge in terms of maximizing oncologic benefits and minimizing toxicity. In the era of precision medicine and more minimal invasive treatment strategies the use of IO therapies in CRC subtypes require further investigation.

\section{The gap of knowledge}

The selection of the appropriate patients that can benefit from minimally invasive therapies is critical in order to maximize the oncologic benefits and minimize risks. Several factors have been identified as predictors of outcome, including tumor size and genetic markers. Although most of these factors are non-modifiable, some can guide technique modification. For example in KRAS-mutant CRC patients with liver metastases the minimal ablation margin needs be larger in order to achieve reasonable local control than what is needed in KRAS wild-type tumors [44-46].

Major complication rates following liver thermal ablation are generally lower than those 
encountered after liver resection, with reported ranges of 4-33\% for radiofrequency and o-19\% for microwave ablations [37,47]. However there is only limited data available on the risk factors that may predispose to complications. Such data might help to personalize treatment.

Radioembolization in chemorefractory patients, performed in salvage setting, has the goal to prolong patients' life. Historical survival at this stage of disease is 5 months, with systemic treatment offering 6 weeks survival benefit $[48,49]$. Median survival after ${ }^{9} \mathrm{Y}$ radiomebolization ranges around 9-12 months [50]. Despite the fact, that such overal survival may seem modest, it still far exceeds overal survival following best supportive care or that achieved with FDA-approved systemic treatments (lonsurf and regorafenib) [51-53]. It is unknown whether combining the FDA-approved agents with ${ }^{9} Y$ radioembolization could further improve survival in this advanced disease stage and this is an area of much needed future investigations.

One of the severe complications of radioembolization is gastrointestinal ulceration. Although this is extremely rare with incidence less than $2 \%$ in most series that have applied proper preparatory arteriography and ${ }^{99 m}$ Tc macroaggregated albumin mapping [54], non-target delivery has been reported in $0-20 \%$ of procedures with a median incidence of $8 \%$ when using advanced antireflux protective techniques [55]. Clearly, there is room to improve safety and patient selection in order to identify the patients who will truly benefit from this salvage treatment the most avoiding unnecessary morbidity.

In addition, there is limited data available about treatment of the patients with metastatic disease and limited curative treatment options, such as those who are not amenable for resection or thermal ablation. ${ }^{9} \mathrm{Y}$ radiation segmentectomy is an emerging radioembolization approach, intended for selective delivery of an ablative radiation dose to achieve complete tumor eradication. However, to date the data about radiation segmentectomy outcomes in the patients with metastatic disease in very scarce and requires further investigation.

The aim of this thesis is to identify predictive factors in order to improve safety and outcomes of image guided, thermal ablative and radioembolization therapies in patients with liver and lung metastases from CRC.

The two main study aims are:

a. Define the factors that can predict local tumor control while minimizing the incidence of complications in patients with hepatic and pulmonary CRC metastases managed with thermal ablation; b. Create a predictive nomogram to improve selection of the patients with refractory CRC liver metastases for ${ }^{\circ} \mathrm{Y}$ radioembolization and to assess ${ }^{\circ} \mathrm{Y}$ radiation segmentectomy outcomes.

\section{OUTLINE OF THIS THESIS}

Chapter 2. Liver-directed and Systemic Therapies for Colorectal Cancer Liver Metastases This literature review provided an overview on the role of different local and systemic therapies to treat metastatic CRC in the liver, including IO therapies. It also overviewed new treatment modalities on the pipeline and key driving mutations which are important for better patient selection.

Chapter 3. Factors Associated with Local Tumor Control and Complications after Thermal Ablation for Colorectal Cancer Liver Metastases: a 15-year Retrospective Cohort Study

This study was conducted to analyze the incidence of local tumor progression as well as complications following thermal ablation of colorectal liver metastases and to identify potential predictive factors.

Chapter 4. Factors Affecting Oncologic Outcomes of ${ }^{90} Y$ Radioembolization of Heavily PreTreated Patients with Colon Cancer Liver Metastases

The aim of this study was to analyze metabolic, anatomic, laboratory, pathologic, primary disease-related and genetic biomarkers, procedure-related factors as well as preand post-radioembolization therapies, that can be associated with liver progression-free and overall survivals. In addition, our goal was to create and internally validate a 1-year overall survival prognostic nomogram using pre-treatment patient characteristics.

Chapter $5 \cdot{ }^{9 \circ} Y$ Resin Microspheres Radioembolization for Colon Cancer Liver Metastases Using Full-Strength Contrast Material

This work was undertaken to specifically address the safety and efficacy of radioembolization with ${ }^{\circ} \mathrm{Y}$ resin microspheres for $\mathrm{CRC}$ liver metastases using undiluted non-ionic contrast material in both "B" and " $\mathrm{D}$ " infusion lines for real-time microspheres infusion monitoring.

Since neither the ${ }^{90} \mathrm{Y}$ microspheres, nor the vehicle used during this infusion are radiopaque, the standard sandwich infusion technique does not allow continuous 
monitoring of the microsphere flow toward the target tumor. This limits the ability to detect microspheres' stasis or reflux, which can result in non-target delivery with unnecessary toxicity, as well as diminished radioactivity reaching the target tumor. This modified infusion technique can potentially decrease incidence of stasis, reflux and non-target delivery.

Chapter 6. Radiation Segmentectomy of Hepatic Metastases with ${ }^{\circ} Y$ Glass-Based Microspheres: Technical Considerations, Correlations to Dosimetry and Preliminary Results

The aim of this study was to evaluate safety and efficacy of ablative-dose radiation segmentectomy with ${ }^{\circ} Y$ glass-based microspheres in patients with limited metastatic liver disease not amenable to surgery or percutaneous ablation.

Chapter 7. Image-Guided Thermal Ablation for the Treatment of Pulmonary Malignancies

This literature review focused on reviewing a variety of image-guided thermal ablation techniques for patients with oligometastatic lung disease and non-small cell lung carcinoma.

Chapter 8. Microwave Ablation in the Management of Colorectal Cancer Pulmonary Metastases Thermal ablation has shown safety and efficacy in the treatment of secondary lung malignancies in non-surgical candidates. However, local progression remains a limitation for these therapies.

This study assessed oncologic outcomes and complications of MW ablation in the management of patients with colorectal pulmonary metastases as well as factors affecting these outcomes.

\section{REFERENCES}

1. Siegel, R. L., Miller, K. D. \& Jemal, A. Cancer statistics, 2018. CA: a cancer journal for clinicians $68,7-30$, doi:10.3322/caac.21442 (2018).

2. Schoenberg, S. O., Attenberger, U. I., Solomon, S. B. \& Weissleder, R. Developing a Roadmap for Interventional Oncology. The oncologist 23, 11621170, doi:10.1634/theoncologist.2017-0654 (2018).

3. Gillams, A. et al. Thermal ablation of colorectal live metastases: a position paper by an international panel of ablation experts, The Interventional Oncology Sans Frontieres meeting 2013. European radiology 25, 3438-3454, doi: 10.1007/soo330-0153779-z (2015).

Ruers, T. et al. Local Treatment of Unresectable Colorectal Liver Metastases: Results of a Randomized Phase II Trial. Journal of the National Cancer Institute 109, doi:10.1093/jnci/djxo15 (2017).

5. Ruers, T. et al. Radiofrequency ablation combined with systemic treatment versus systemic treatmen alone in patients with non-resectable colorectal liver metastases: a randomized EORTC Intergroup phase II study (EORTC 40004). Ann Oncol 23, 26192626, doi:10.1093/annonc/mds053 (2012).

6. Hammill, C. W. et al. Outcome after laparoscopic radiofrequency ablation of technically resectable colorectal liver metastases. Annals of surgical oncology 18, 1947-1954, doi: 10.1245/10434-010-15359 (2011).

7. Otto, G. et al. Radiofrequency ablation as first-line treatment in patients with early colorectal liver metastases amenable to surgery. Annals of surgery 251, 796-803, doi:10.1097/SLA.obo13e3181bcgfae (2010).

8. Puijk, R. S. et al. Colorectal liver metastases: surgery versus thermal ablation (COLLISION) - a phase II single-blind prospective randomized controlled trial. BMC cancer 18, 821, doi: 10.1186/s12885-018-47168 (2018).

9. Petrelli, F. et al. Stereotactic body radiotherapy for colorectal cancer liver metastases: A systematic review. Radiotherapy and oncology: journal of the European Society for Therapeutic Radiology and Oncology 129, 427-434, doi:10.1016/j. radonc.2018.06.035 (2018).

10. Van Cutsem, E. et al. ESMO consensus guidelines for the management of patients with metastatic colorectal cancer. Annals of oncology: official journal of the European Society for Medical Oncology 27, 1386-1422, doi:10.1093/annonc/mdw235 (2016).

11. Yoshino, T. et al. Pan-Asian adapted ESMO consensus guidelines for the management of patients with metastatic colorectal cancer: a JSMOESMO initiative endorsed by CSCO, KACO, MOS, SSO and TOS. Annals of oncology: official journal of the European Society for Medical Oncology 29, 447o, doi:10.1093/annonc/mdx738 (2018).

12. Jones, R. P. et al. Neoadjuvant treatment of colorectal liver metastases (CRLM) with drug eluting beads trans-arterial chemoembolization (DEBIRI-TACE): A multi-institute phase II study in resectable metastases. Journal of Clinical Oncology 30, 3613-3613, doi:10.120o/jco.2012.30.15_suppl.3613 (2012)

13. Livraghi, T. et al. Percutaneous radiofrequency ablation of liver metastases in potential candidates for resection: the "test-of-time approach". Cancer 97, 3027-3035, doi:10.1002/cncr.11426 (2003).

14. Wasan, H. S. et al. First-line selective internal radiotherapy plus chemotherapy versus chemotherapy alone in patients with liver metastases from colorectal cancer (FOXFIRE, 
SIRFLOX, and FOXFIRE-Global): a combined analysis of three multicentre, randomised, phase 3 trials. The Lancet. Oncology 18, 1159-1171, doi: 10.1016/ s1470-2045(17)30457-6 (2017).

15. Yamakado, K. et al. Radiofrequency Ablation Combined with Hepatic Arterial Chemoembolization Using Degradable Starch Microsphere Mixed with Mitomycin C for the Treatment of Liver Metastasis from Colorectal Cancer: A Prospective Multicenter Study. Cardiovascular and interventional radiology 40 , 560-567, doi: 10.1007/soo270-016-1547-3 (2017).

16. Wu, Z. B. et al. Percutaneous microwave ablation combined with synchronous transcatheter arterial chemoembolization for the treatment of colorectal liver metastases: results from a follow-up cohort OncoTargets and therapy 9, 3783-3789, doi:10.2147| ott.S105192 (2016).

17. Vogl, T. J. et al. Survival of patients with nonresectable, chemotherapy-resistant colorectal cancer liver metastases undergoing conventional lipiodol-based transarterial chemoembolization (CTACE) palliatively versus neoadjuvantly prior to percutaneous thermal ablation. European journal of radiology 102, 138-145, doi:10.1016/j. ejrad.2018.03.015 (2018).

18. Ishikawa, T. et al. [Multiple liver metastases due to sigmoid colon cancer successfully treated by degradable starch microspheres (DSM)-TAE, radiofrequency ablation therapy, and Uzel/UFT]. Gan to kagaku ryoho. Cancer \& chemotherapy 37, 335-338 (2010)

19. Fong, Z. V. et al. Combined hepatic arterial embolization and hepatic ablation for unresectable colorectal metastases to the liver. The American surgeon 78, 1243-1248 (2012).

20. Wang, D. S., Louie, J. D. \& Sze, D. Y. Intra-arterial therapies for metastatic colorectal cancer
Seminars in interventional radiology 30, 12-20, doi: 10.1055/s-0033-1333649 (2013).

21. Weng, M. et al. Radiofrequency ablation versus resection for colorectal cancer liver metastases: a meta-analysis. PloS one 7, e45493, doi:10.1371 journal.pone.0045493 (2012).

22. Mulier, S. et al. Radiofrequency ablation versus resection for resectable colorectal liver metastases: time for a randomized trial? An update. Digestive surgery 25, 445-46o, doi: 10.1159/0oo184736 (2008).

23. Pathak, S. et al. Ablative therapies for colorectal liver metastases: a systematic review. Colorectal disease: the official journal of the Association of Coloproctology of Great Britain and Ireland 13, e252-265, doi:10.1111|j.1463-1318.2011.02695.X (2011).

24. Petre, E. N. et al. Treatment of pulmonary colorectal metastases by radiofrequency ablation. Clinical colorectal cancer 12, 37-44, doi:10.1016/j. clcc.2012.07.003 (2013).

25. Mouli, S. K., Kurilova, I., Sofocleous, C. T. \& Lewandowski, R. J. The Role of Percutaneous ImageGuided Thermal Ablation for the Treatment of Pulmonary Malignancies. AJR. American journal of roentgenology 209, 740-751, doi:10.2214/aj.17.18368 (2017).

26. Nikfarjam, M., Muralidharan, V. \& Christophi, C. Mechanisms of focal heat destruction of liver tumors. The Journal of surgical research 127, 208223, doi:10.1016/j.jss.2005.02.009 (2005).

27. Gillams, A. R. \& Lees, W. R. Radio-frequency ablation of colorectal liver metastases in 167 patients. European radiology 14, 2261-2267, doi: 10.1007/so0330-004-2416-z (2004).

28. Siperstein, A. E., Berber, E., Ballem, N. \& Parikh, R. T. Survival after radiofrequency ablation of colorectal liver metastases: 10-year experience. Annals of surgery 246, 559-565; discussion 565-557, doi:10.1097| SLA.obo13e318155äb6 (2007).
29. Veltri, A. et al. Radiofrequency ablation of colorectal liver metastases: small size favorably predicts technique effectiveness and survival. Cardiovascular and interventional radiology 31 , 948-956, doi: 10.1007/soo270-008-9362-o (2008).

30. Gillams, A. R. \& Lees, W. R. Five-year survival in 309 patients with colorectal liver metastases treated with radiofrequency ablation. European radiology 19, 1206-1213, doi: 10.1007/s00330-008-1258-5 (2009).

31. Sofocleous, C. T. et al. CT-guided radiofrequency ablation as a salvage treatment of colorectal cancer hepatic metastases developing after hepatectomy Journal of vascular and interventional radiology: JVIR 22, 755-761, doi:10.1016/j.jvir.2011.01.451 (2011).

32. Solbiati, L. et al. Small liver colorectal metastases treated with percutaneous radiofrequency ablation: local response rate and long-term survival with up to 10-year follow-up. Radiology 265, 958968, doi:10.1148/radiol.12111851 (2012).

33. Shibata, T., Niinobu, T., Ogata, N. \& Takami, M. Microwave coagulation therapy for multiple hepatic metastases from colorectal carcinoma. Cancer 89, 276-284 (2000).

34. Tanaka, K. et al. Outcome after hepatic resection versus combined resection and microwave ablation for multiple bilobar colorectal metastases to the liver. Surgery 139, 263-273, doi:10.1016/j. surg.2005.07.036 (2006).

35. Shady, W. et al. Percutaneous Microwave versus Radiofrequency Ablation of Colorectal Liver Metastases: Ablation with Clear Margins (Ao) Provides the Best Local Tumor Control. Journal of vascular and interventional radiology: JVIR 29, 268275.e261, doi:10.1016/j.jvir.2017.08.021 (2018).

36. Liang, P. et al. Prognostic factors for percutaneous microwave coagulation therapy of hepatic metastases.AJR.American journal of roentgenology 181, 1319-1325, doi:10.2214/ajr.181.5.1811319 (2003).
37. Vogl, T. J . et al. Thermal ablation of liver metastases from colorectal cancer: radiofrequency, microwave and laser ablation therapies. Radiol Med 119, 451461, doi: 10.1007/ s11547-014-0415-y (2014).

38. Sartori, S., Di Vece, F., Ermili, F. \& Tombesi, P. Laser ablation of liver tumors: An ancillary technique, or an alternative to radiofrequency and microwave? World journal of radiology 9, 91-96, doi:10.4329/wjr. vg.i3.91 (2017)

39. Sartori, S., Tombesi, P. \& Di Vece, F. Thermal ablation in colorectal liver metastases: Lack of evidence or lack of capability to prove the evidence? World journal of gastroenterology 22,3511-3515, doi:10.3748| wjg.v22.i13.3511 (2016).

40. Park, M. Y. et al. Preliminary experience using high intensity focused ultrasound for treating liver metastasis from colon and stomach cancer. International journal of hyperthermia : the official journal of European Society for Hyperthermic Oncology, North American Hyperthermia Group 25, 180-188, doi:10.1080/02656730802641949 (2009).

41. Shyn, P. B. et al. Percutaneous imaging-guided cryoablation of liver tumors: predicting local progression on 24-hour MRI. AJR. American journal of roentgenology 203, W181-191, doi:10.2214/ ajr.13.10747 (2014).

42. Petre, E. N. \& Sofocleous, C. Thermal Ablation in the Management of Colorectal Cancer Patients with Oligometastatic Liver Disease. Visceral medicine 33, 62-68, doi: 10.1159/000454697 (2017).

43. Kurilova, I. et al. Factors Affecting Oncologic Outcomes of goY Radioembolization of Heavily Pre-Treated Patients With Colon Cancer Liver Metastases. Clinical colorectal cancer 18, 8-18, doi:10.1016/j.clcc.2018.08.004 (2019).

44. Shady, W. et al. Kras mutation is a marker of worse oncologic outcomes after percutaneous radiofrequency ablation of colorectal liver 
metastases. Oncotarget 8, 66117-66127, doi:10.18632| oncotarget.19806 (2017).

45. Odisio, B. C. et al. Local tumour progression after percutaneous ablation of colorectal liver metastases according to RAS mutation status. The British journal of surgery 104, 760--768, doi:10.1002/ bjs.1049o (2017).

46. Calandri, M. et al. Ablation of colorectal liver metastasis: Interaction of ablation margins and RAS mutation profiling on local tumour progression-free survival. European radiology 28 2727-2734, doi: 10.1007/soo330-017-5273-2 (2018).

47. Sorensen, S. M., Mortensen, F. V. \& Nielsen, D. T. Radiofrequency ablation of colorectal liver metastases: long-term survival. Acta radiologica (Stockholm, Sweden: 1987) 48, 253-258, doi: 10.1080/02841850601161539 (2007)

48. Mayer, R. J. et al. Randomized Trial of TAS-102 for Refractory Metastatic Colorectal Cancer. 372, 19091919, doi: 10.1056/NEJMoa1414325 (2015).

49. Grothey, A. et al. Regorafenib monotherapy for previously treated metastatic colorectal cancer (CORRECT): an international, multicentre, randomised, placebo-controlled, phase 3 trial. Lancet (London, England) 381, 303-312, doi: 10.1016 so140-6736(12)61900-x (2013).

50. Sofocleous, C. T. et al. Phase I trial of selective internal radiation therapy for chemorefractory colorectal cancer liver metastases progressing after hepatic arterial pump and systemic chemotherapy. Clinical colorectal cancer 13, 27-36, doi:10.1016/j. clcc.2013.11.010 (2014).

51. Lewandowski, R. J. et al. Twelve-year experience of radioembolization for colorectal hepatic metastases in 214 patients: survival by era and chemotherapy. European journal of nuclear medicine and molecular imaging 41, 1861-1869, doi: $10.1007 / 500259-014-2799-2$ (2014)
52. Kennedy, A. S. et al. Multicenter evaluation of the safety and efficacy of radioembolization in patients with unresectable colorectal liver metastases selected as candidates for $(90) Y$ resin microspheres. Journal of gastrointestinal oncology 6, 134-142, doi:10.3978/j.issn.2078-6891.2014.109 (2015).

53. Saxena, A. et al. Is yttrium-go radioembolization a viable treatment option for unresectable, chemorefractory colorectal cancer liver metastases? A large single-center experience of 302 patients. Annals of surgical oncology 22, 794-802, doi: 10.1245/1510434-014-4164-x (2015).

54. van Hazel, G. A. et al. SIRFLOX: Randomized Phase III Trial Comparing First-Line mFOLFOX6 (Plus or Minus Bevacizumab) Versus mFolfoX6 (Plus or Minus Bevacizumab) Plus Selective Internal Radiation Therapy in Patients With Metastatic Colorectal Cancer. Journal of clinical oncology: official journal of the American Society of Clinical Oncology 34, 1723-1731, doi:10.120o/jco.2015.66.1181 (2016)

55. Murthy, R. et al. Gastrointestinal complications associated with hepatic arterial Yttrium-go microsphere therapy. Journal of vascular and interventional radiology : JVIR 18, 553-561; quiz 562, doi:10.1016/j.jvir.2007.02.002 (2007). 


\section{PART 1}

Management of Colorectal Liver Metastases In Interventional Oncology Setting 
Liver-directed and Systemic Therapies for Colorectal Cancer Liver Metastases

Nancy Kemeny*, Ieva Kurilova*, Jia Li*, Juan Camacho, Constantinos T. Sofocleous *Joint co-authors due to equal contribution to this work 


\section{EPIDEMIOLOGY, PREDISPOSING FACTOR AND PATHOLOGY}

Colorectal cancer (CRC) is the third most common cancer worldwide, resulting in an estimated 140,250 new cases and 50,630 deaths in 2018 [1,2]. Liver is the most common visceral metastatic site due to the portal venous drainage from the colon. About $15 \%$ of patients present with synchronous liver metastases at initial diagnosis, which is an independent poor prognostic factor [3]. Approximately 50\% of patients ultimately develop liver metastasis during the course of the disease.

The risk factors for CRC are classified to non-modifiable (age, family history, hereditary predisposition, inflammatory bowel disease and adenomatous polyp) and modifiable (diets, smoking, obesity and high alcohol consumption) [4].

Left-sided and right-sided CRCs harbor different clinical and biologic characteristics with different exposures to potential carcinogenic toxins and microbiota, which can potentially impact the prognosis. Right-sided primary CRCs are more likely to have genome-wide hypermethylation via the $\mathrm{CPG}$ island methylator phenotype (CIMP), hypermutated state via microsatellite instability, BRAF mutation [5], greater proportions of the "microsatellite unstable/immune" CMS1 and the "metabolic" CMS 3 consensus molecular subtypes. Molecular tumor subtypes (different from primary tumor) have been defined for colorectal liver metastases (CLM) [6] and impact prognosis.

KRAS mutation is detected in $35-45 \%$ of CRCs and it is a strong negative prognostic biomarker (associated with more infiltrating/migratory characteristics of cancer cells [7]) and a negative predictive biomarker in terms of resistance to anti-EGFR treatment [8], higher incidence of positive and narrow margins at surgery [9] and worse oncologic outcomes after ablation of CLM [10,11]. BRAF-mutant cancers, comprising 10\% of all CRCs, represent a distinct subset of CRC with its own clinical implications with regard to prognosis, treatments and emerging therapeutic strategies. BRAF-mutant CRCs tend to be microsatellite instable (MSI-high), mucinous histology, poorly differentiated, less likely to have metastatic disease amenable for surgical resection as well as poorer overall survival (OS) [12]. Approximately, 15-20 \% of colorectal cancers display MSI with prognostic and therapeutic implications as these tumors are highly immunogenic and can be targeted with immunotherapy [13].

Main limitation of the studies, analyzing outcomes of CLM include heterogeneity in terms of prior therapies (treatment-naïve, patients after first-, second-, or subsequent treatment lines) and tumor subtypes.
Summary of the most important facts about liver-directed and systemic therapies for CLM is presented in Tables 1A-B.

Table 1A Liver-directed therapies for colorectal cancer liver metastases

\section{Colorectal cancer (CRC) is the third most common cancer worldwide. Liver is the} with synchronous metastasis being an independent poor prognostic factor [3]

Genetic molecular profles notonly have impact on prognosis but also provide therapeutic values KRAS met is detect cancers comprise $10 \%$ of all CRCs and associated with poorer overall survival

3. Systemic chemotherapies are proven to have survival benefit in all clinical settings from postliver metastatectomy,

4. Cytotoxic chemotherapies remain the mainstream therapy for metastatic CRC, even in patients with resectab or amenable to ablation disease. Chemotherapy combined with
significantly improve outcomes in this patient population [14]

Treaplo

-Treatment options for liver-only or dominant metastasis include surgical resection/ablation, loco-regional as well a i inmunotherapy, with immune checkpoint inhibitors benefiting small percentage of patients whore tumor is MMR deficient

6. When liver metastases are completely resected and/or ablated, long-term survival and potential cure could be achieved. Clinical Risk Score (CRS) and margins impact surgical outcomes and potential for cure $[15,16]$

7. Despite a relevantly high recurrence rate following resection and or ablation of liver metastases, ablation (with outcomes of ablation similarly to surgery. Ablation with margins over $10 \mathrm{~mm}$ offers the best local tumor control $[17,19]$

8. Intra-arterial therapies and in particular Y-go radioembolization is recommended for CLM treatment in the salvage setting with liver disease progression while on or after second line chemotherapy with encouraging oncologic outcomes $[20]$

9. Liver-directed chemotherapy through hepatic arterial pump demonstrated survival benefit in multiple clinical settings including adjuvant, neoadjuvant and metastatic

. Loco-regional interventions are also being combined with different systemic therapies to enhance anti-tumo effect and to facilitate drug delivery

Table 1B Liver-directed therapies for colorectal cancer liver metastases

1. There are estimated 140,250 new cases of CRC, and 50,630 patients will die from CRC in 2018 in the USA 15\% patients present with synchronous liver metastases, $50 \%$ patients with CRC will ultimately develop liver metastasis

Only around 15-20\% of the patients with CLM are eligible for liver resection

4. The recurrence rate following resection of liver metastases from CRC is in the order of $60-70 \%$

5. Up to $38 \%$ patients with initially unresectable liver metastases could have disease converted to surgically resectable after receiving proper therapy

3 major pivotal studies for the last 5 years Trial. Journal of the National Cancer Institute 109, doi:10.1093/jnci/djxo15 (2017) Groot Koerkamp B, Sadot E, Kemeny NE, et al. Perioperative hepatic arteri 2,368 consecutive patients. J Clin Oncol. 2017335:1938-1944 


\section{Table $1 B$ continued.} Wasan HS, Gibbs P, Sharma NK, Taieb J, Heinemann V, Ricke J, et al. First-line selective internal radiotherapy
plus chemotherapy versus chemotherapy alone in patients with liver metastases from colorectal cancer (FOXFIRE, SIRFLOX, and FOXFIRE-Global): a combined analysis of three multicentre, randomised, phase 3 trials. The Lancet Oncology. 2017;18(9):1159-71

1. $\quad$ All metastatic colorectal cancer with liver metastasis should be managed by multidisciplinary teams including medical oncologist, surgical oncologist, interventional radiologist as well as diagnostic radiologist

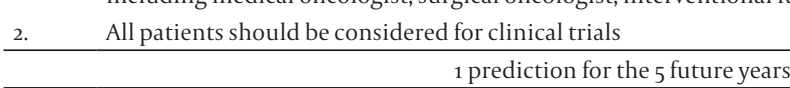

Combination of loco-regional plus systemic therapies might be the leading approach in this patient population in an effort to prolong overall survival

\section{DIAGNOSIS AND INITIAL WORK-UP}

Most primary CRCs are diagnosed through colonoscopy either through regular screening or due to symptoms such as change in bowel habits, GI bleeding (hematochezia, melena, occult blood loss and iron deficiency anemia) or abdominal pain. Some cases are diagnosed due to signs or symptoms caused by metastatic disease.

CRCs can spread by lymphatic and hematogenous dissemination, as well as contiguous and transperitoneal spread. The most common metastatic sites are regional lymph nodes, liver, lung and bones.

This article reviews management of CLM. The only potentially curative treatment option for CLM is surgical resection and/or complete ablation [15]. Recent advances in chemotherapy, surgical and interventional techniques allow a subset of initially unresectable CLMs to be downsized to resection or ablation. Thus precise assessment of the extent of disease is critical to determine the resectability. Several imaging modalities are used to identify CLM. Commonly used imaging modalities are ultrasound, computed tomography (CT), magnetic resonance imaging (MRI), ${ }^{18} \mathrm{~F}$-FDG PET/CT and more recently fully integrated ${ }^{18} \mathrm{~F}$-FDG PET/MR. The utility of different imaging modalities is described in Table 2.

Our recommendation of working up CLMs is to start with CT chest/abdomen/pelvis with triphasic liver protocol. If the patient is potentially resectable, liver MRI should be the next step to further define the extent of disease to ensure a proper selection for surgery. ${ }^{18} \mathrm{~F}-\mathrm{FDG}$ PET/CT is also advisable especially prior to resection or ablation of metastatic disease [26].
Table 2 Imaging modalities used for colorectal liver metastases (CLM) detection and follow-up

Imaging modality

CT of the chest/ abdomen/ pelvis with triphasic liver protocol

MRI with liver-specific contrast agents and diffusion-weighted imagin

s8F-FDG PET/CT imaging

sF FDG PET/MRI imaging

Traditional ultrasound (US), contrastTraditional thasound (US), contrastoperative US

\section{Description}

It is the most commonly used imaging modality in diagnosis of both intrahepatic and extrahepatic metastases as well as post-treatment followup [21-23]; however, CT is not sensitive enough to detect lesions smaller tha

It is more sensitive than CT for detecting smaller CLM, it is more often chosen by surgeons when planning metastatectomy [21-23]

It is not routinely used in CRC work-up, however, if CT or MRI detects suspicious but inconclusive abnormalities, "if-FDG PET/CT may be considered. It is recommended for restaging prior to resection or ablation
of metastatic disease It is gaining some populatist

It is gaining some popularity for its sensitivity and specificity in diagnosing

They all have a role in detecting CLM [25], especially useful for image-guided needle biopsy of CLM

\section{APPROVED/RECOMMENDED TREATMENTS}

Liver resection is considered to be a "gold standard" for CLM treatment, with median OS in liver resected patients of $28-46$ months [27]. However, only $15 \%-20 \%$ of the patients are considered to be eligible for liver surgery $[28,29]$. Patients with oligometastatic disease (OMD), located in anatomically favorable territory and low tumor burden may be considered to have upfront liver metastatectomy, followed by 6-months adjuvant therapy. National Comprehensive Cancer Network (NCCN) guidelines recommend surgery or ablation alone or in combination as long as all visible metastases can be eradicated [26].

When liver metastases are completely resected and/or ablated, long-term survival and potential cure could be achieved. In patients who can undergo liver resection followed by adjuvant systemic therapy plus HAI, 5-year OS as high as $78 \%$ can be achieved [30], which is significantly better than 5-year OS of the patients treated with chemotherapyalone, which is around 10\%. Clinical Risk Score (CRS) and margins impact surgical outcomes and potential for cure $[15,16]$.

Cytotoxic chemotherapies remain the mainstream therapy for metastatic CRC, even in patients with resectable or amenable to ablation disease. Chemotherapy combined with resection or ablation of all metastatic sites significantly improves outcomes in this patient population [14]. 
Adjuvant therapies after CLM resection

Adjuvant therapy options following liver resection include systemic chemotherapy with FOLFOX or 5-FU/LV [31] and hepatic arterial infusion chemotherapy (HAI) with floxuridine (FUDR) alone or in combination with systemic chemotherapy in certain institutions.

HAI FUDR demonstrated liver progression-free survival (PFS) and OS benefit, based on four randomized trials in the adjuvant setting [32-35] (please see Supplement 1 for detailed HAI data). NCCN guidelines conclude that HAI infusion with or without systemic chemotherapy is an option as adjuvant or peri-operative therapy in the setting of liver metastatectomy. In addition to FUDR, several other chemotherapeutic agents can be administered through HAI, including 5-FU, mitomycin, oxaliplatin and irinotecan [36-38]. However, due to the complexity of hepatic pump placement, management of HAI pump and administration of chemotherapy via HAI, this approach has only been utilized in certain institutions with relevant expertise.

\section{Neoadjuvant conversion therapy}

Among patients with CLM, up to $30 \%$ of initially unresectable patients could have disease converted to surgically resectable after receiving systemic therapy. The options of conversion therapy are as follows:

Systemic conversion therapies include the following:

- FOLFOX or CAPOX +/- bevacizumab: for the patients without prior oxaliplatin exposure [39-41]. Bevacizumab has modest benefit when in combination of FOLFOX or XELOX in the peri-operative setting but with potential complications such as arterial thromboembolic events, hemorrhage, delayed wound healing, gastrointestinal perforation, biliary complications, or severe hypertension. Its use in the peri-operative setting requires caution; in general, it is recommended avoiding bevacizumab 4 weeks before and after invasive operations [41].

- FOLFIRI +/- cetuximab or panitumumab: for the patients with prior oxaliplatin as adjuvant therapy, left sided primary cancer and wild-type KRAS [42-44];

- FOLFOXIRI +/- bevacizumab. This regimen is generally used in younger, healthier patients; thus the patients with high tumor burden, risk of chemotherapy-related toxicity should be under consideration when choosing this regimen. This regimen was associated with conversion rate of up to $40 \%$ with significantly prolonged OS [45-48].
Locoregional therapies include the following:

- HAI therapy alone or with systemic chemotherapy. HAI with FUDR plus systemic chemotherapy (FOLFOX, FOLFIRI) as conversion therapy resulted in CLM resectability in nearly $50 \%$ of the patients $[49,50]$.

- $\quad$ Neoadjuvant ${ }^{90} Y$ radiation lobectomy [51] or transarterial chemoembolization with drug-eluting beads (DEBIRI-TACE) [52].

\section{Unresectable CLMs}

If complete resection /ablation of CLM is not feasible or patients are not fit surgical candidates, other locoregional therapies, such as HAI, percutaneous ablation, chemoembolization, radioembolization (RE), and radiation therapy (RT), including stereotactic body RT (SBRT) can all be considered for the patients with OMD. Also, in selected patients percutaneous ablation within the "test of time" concept maybe preferable to surgery as the initial local cure while observing the disease biology in potentially resectable patients. In the event of local failure in the ablation site, in patients without multifocal progression during the follow - up period surgery remains a subsequent option. "Test of time" allows expression of disease biology and can spare patients with aggressive tumor biology the morbidity of a non-beneficial surgery [53]. Percutaneous ablation can also be useful for disease control without systemic chemotherapy administration in selected patients, allowing for "chemotherapy holiday"

Systemic palliative therapies

For this category, goals of therapy focus on palliative and non-curative measures with expectation of maintaining quality of life and potentially prolonging OS. Many combinations are available, consisting of cytotoxic chemotherapeutic agents including irinotecan, oxaliplatin, capecitabine; monoclonal antibodies targeting VEGF and EGFR pathways such as bevacizumab, ramucirumab, cetuximab, panitumumab; tyrosine kinase inhibitor regorafenib, recombinant fusion protein aflibercept, and trifluridinetipiracil, as well as immunotherapy. Overall principle is to expose patients to all active agents sequentially if they can tolerate. Enrollment in a clinical trial is always recommended in this setting.

First-line and beyond first line palliative locoregional and systemic treatment options for the patients with CLM are described in Table 3. 
Table 3 Palliative treatment options for the patients with colorectal liver metastases, adopted from several current guidelines. TACE- transarterial chemoembolization, TARE- transarterial radioembolization

\begin{tabular}{ll}
\hline Treatment regimen & Description \\
\hline $\begin{array}{ll}\text { FOLFOX and FOLFIRI +|- bevacizumab line systemic palliative chemotherapy options } \\
\text { or cetuximab }[54,55]\end{array}$ & $\begin{array}{l}\text { Left-sided wild-type KRAS colorectal cancer (CRC) patients have significant } \\
\text { survival benefit from cetuximab, while right-sided CRC patients have survival } \\
\text { benefit from bevacizumab [56] } \\
\text { Combining both anti-VEGF and anti-EGFR agents is not recommended due to }\end{array}$
\end{tabular}

poorer outcome [57,58].

For the patients with RAS wild-type tumor [59, 6o]

S-1 is only used in selected countries, such as Japan and Korea [61, 62]

FOLFIRINOX or FOLFOXIRI This regimen is used only in selected younger patients with high tumor burden and RAS or BRAF mutation

HAI with FUDR or in combination with For patients with unresectable liver metastasis [49,50] FU/LV, FOLFOX or FOLFIRI \begin{tabular}{ll}
\hline Irinotecan-based chemotherapy & $\begin{array}{l}\text { For patients received oxaliplatin based initial therapy, irinotecan-based } \\
\text { chemotherapy is considered to be the next treatment choice. } \\
\text { The following regimens are recommended: }\end{array}$
\end{tabular} FOLFIRI

FOLFIRI + bevacizumab (for patients with tumor bearing RAS mutation) FOLFIRI + cetuximab/ panitumumab (for patients with RAS wild-type tumor) FOLFIRI with intravenous aflibercept $[63,64]$ FOLFIRI with ramucirumab [65]

Oxaliplatin-based chemotherapy $\quad$ For patients received irinotecan-based initial therapy, changing to oxaliplatinbased chemotherapy with or without biological agents depending on RAS

mutational status would be reasonable
For RAS wild-type, anti-EGFR therapy would be appropriate, anti-VEGF agent can be also be considered, although only anti-VEGF agent would be indicated if RAS mutations present

Regorafenib or trifluridine-tipiracil For the patients who have received and failed both oxaliplatin-and irinotecanbased be offered for additional therapy based on performance status and organ functions, we sagest HAI therapy rening Mismatch repair protein (MMR) deficient or MSI-high tumors may benefit

HAl therapy from check point inhibitors

Patients with MMR-deficient metastatic CRC had a 50\% objective response rate (ORR) and a $89 \%$ disease control rate (DCR) when treated with pembrolizuma while in contrast, the ORR and DCR were o and 16\% respectively in MMRproficient mCRC [69, 70]

Similar results were also seen in CheckMate-142, in which patients received nivolumab with or without ipilimumab. Significantly higher ORR and PFS were demonstrated in MMR-deficient MCRC [71]

Reutilizing the regimen initially used in During the often lengthy phase of sequential therapy, tumors may regain

\begin{tabular}{cc} 
the treatment sequence & sensitivity to the previously used agents. \\
\hline Locoregional treatment options
\end{tabular}

HAI therapy $\quad$ For liver-dominant metastatic CRC (early stage multifocal liver-only disease)

TACE, TARE $\quad$ For liver-dominant metastatic CRC ( late stages multifocal liver-only disease)

\section{ROLE OF INTERVENTIONAL ONCOLOGY /RADIOLOGY (IO/IR)}

The main focus of IO/IR in metastatic CRC ( $\mathrm{mCRC}$ ) is OMD. It is characterized by disease localization to a few sites and tumors allowing the option to use local and ablative treatments (LAT) aiming to improve disease control and therefore clinical outcome in these patients [72]. Although the mortality of CRC patients has dramatically decreased in the last 20 years following the introduction of new systemic treatments, the management of mCRC remains a major challenge with surgical resection and ablation being the only potentially curative options.

IO/IR plays an important role in the management of mCRC from disease diagnosis through tissue sampling, treatment of unresectable OMD, bridging potentially resectable patients or treating chemorefractory metastatic disease in the salvage setting [73] to palliation. Therefore, there is a strong international consensus that the interventional oncologist/radiologist should be a standing member of the institutional tumor board [74].

Most frequent clinical scenarios for LAT include the following:

Neoadjuvant setting. In this setting LAT can be used as:

- Potentially curative treatment for unresectable patients with limited metastatic tumor burden;

- Induction therapy in order to downsize tumor in potentially resectable patients [52];

- Percutaneous ablation can be applied within the "test of time" concept, when it is used instead of surgery in order to observe the disease biology in potentially resectable patients [53].

Adjuvant setting. In this setting LAT can be used for:

- Combination with first-line chemotherapy in carefully selected patients [52, 75];

- $\quad$ Treatment of CLM by means of combined percutaneous and endovascular therapies [76-8o];

- Induction of distant tumor response by combining LAT with immunotherapy;

- Disease control without systemic chemotherapy administration in selected patients, allowing for "chemotherapy holiday".

Salvage setting: for chemorefractory patients with a goal to improve disease control and OS with minimal impact on the quality of life [81]. 
Percutaneous ablative techniques

Percutaneous ablative techniques include a wide range of modalities, which are divided into two groups:

- Thermal modalities (heat- and cold-based). They include radiofrequency (RFA) [8287], microwave (MWA) [17, 88-90], cryoablation [91], laser ablation [92-94] and high intensity focused ultrasound [95];

- Non-thermal modality, which include irreversible electroporation [96-98].

RFA is the most extensively studied ablation modality with multiple larger retrospective case matched comparisons as well as meta-analyses available, comparing RFA to surgical resection or using RFA in addition to systemic chemotherapy [14, 99-104].

For treatment of CLM, hepatocellular carcinoma algorithm of the West and Japan is often applied, which recommends RFA for $<3 \mathrm{~cm}$ liver metastases in unresectable patients with $\leq 3$ tumors [105-107]. However, for CLM the lesion number is not an absolute limiting factor for RFA, if successful treatment of all metastases can be accomplished, with most centers preferentially treating patients with $\leq 5$ lesions. Several studies demonstrated highest rates of complete tumor destruction with RFA for lesions of $\leq 3 \mathrm{~cm}$ [108-110], with oncological outcomes similar to surgical resection $[18,108,111-114]$.

Recent thermal ablation literature focuses on improving the relatively high local tumor recurrence rates $(2 \%-60 \%)[17,86,115-117]$. This can be achieved by creating sufficient (ideally >10 mm) minimal ablation margins ("Ao ablation" concept) [17, 118], incorporating metabolic image-guidance for tumor ablation by means of ${ }^{18} \mathrm{~F}$ FDG-PET [119] as well as stratifying the patients based on modified clinical risk scores and genetic mutation profile, such as KRAS mutation $[11,18,19,86,118,120]$. Essentially ablation with margins of $>10 \mathrm{~mm}$ are associated with few if any local failures $[17,18]$. Similarly ablation margins of $>5 \mathrm{~mm}$ with immediate post-ablation zone center and margin biopsy confirming complete tumor necrosis offered over 97\% local progression-free survival 30 months post-RFA [19].

The CLOCC trial is extremely important as it the only randomized-controlled trial (RCT), providing the evidence of OS advantage when using LAT (by means of RFA) in combination with systemic chemotherapy vs. systemic chemotherapy alone, with OS at 8 years of $36 \%$ and $8 \%$, respectively $[14,102]$. However, due to trial's limitations, new multicenter prospective COLLISION trial has been initiated, comparing thermal ablation (TA) and liver resection outcomes for CLM [121]. The main hypothesis is that TA might enable to achieve similar local tumor control and OS rates to surgical resection while reducing morbidity, mortality, direct economic costs, hospitalization days and improving quality of life [121]. Achieving similar local tumor control and OS with TA compared to surgery, will further establish the terms "resectable" and "ablatable" as synonyms.

The outcomes following RFA and MWA in term of safety and toxicity are comparable. Although there is data on MWA superiority than RFA for CLM [122], when stratified by margin size there was no difference in local tumor progression-free survival [17] with no local tumor progression for tumors ablated with margins $>10 \mathrm{~mm}$. The latest generation MWA systems offer technical advantages such as greater intra-tumoral temperature, deeper penetration of energy, propagation across the poorly conductive tissues, less sensitivity to the heat-sink effect, and larger ablation volume, enabling to treat larger tumors with adequate safety margin when compared to RFA [123-125].

Endovascular approaches

While ablation and resection are the only potentially curative options for mCRC, only around 20\% of CLM patients are eligible for these treatments. Endovascular approaches, such as transarterial chemoembolization (TACE) [126-132] and 9 ${ }^{\circ} Y$ radioembolization (RE) $[26,75,133-141]$ demonstrated improvement in OS and quality of life in chemorefractory CLM.

$R E$

${ }^{90} \mathrm{Y} R E$ is an FDA approved liver brachytherapy, recommended through the NCCN and the ESMO guidelines for the treatment of CLM in the salvage setting with liver disease progression while on or after second line chemotherapy with encouraging oncologic outcomes $[26,72,75,133,136,137,142-153]$. It is also used to treat unresectable noncolorectal liver metastases [154-156]. However, when the patients present to the RE in salvage setting with advanced tumor load, unfavorable biological tumor characteristics and comorbidities, the range of outcomes post-RE is highly variable, with objective response rates varying between $10 \%-48 \%$ when RE is applied in the third and subsequent chemotherapy regiment setting $[146,152,157,158]$.

The main effect of RE is attributed to radiation as it has a minimal embolic effect. Three types of particles are currently being used for RE: ${ }^{\circ} \mathrm{Y}$ microspheres (resin or glass-based) and ${ }^{166} \mathrm{Ho}$ microspheres.

${ }^{\circ} \mathrm{Y}$ microspheres are the most commonly used microspheres for RE. ${ }^{\circ} \mathrm{Y}$ is $\beta$-emitter, with $96 \%$ of radiation delivered within 12 days in the tumor, with up to $1-\mathrm{cm}$ penetration depth 
around each microsphere, enabling to achieve treatment margins around the tumor, similarly to surgical resection or thermal ablation [159].

Physical properties of glass-based and resin microspheres are different due to the size and number of the particles required to deliver the same radiation dose. RE with resin microspheres enables to deliver higher number of microspheres with potentially more homogeneous tumor coverage with a risk of embolic effect and stasis prior to total dose delivery $[133,160,161] .{ }^{\circ} \mathrm{Y}$ microspheres are not radiopaque, making realtime infusion monitoring challenging. Modified infusion methods using diluted or undiluted contrast medium have been explored with resin microspheres and resulted in decrease incidence of stasis as well as fluoroscopy time [153, 162]. No substantial difference between the oncological outcomes when using resin or glass microspheres for CLM have been shown [20].

${ }^{166} \mathrm{Ho}$ microspheres emit $\gamma$-radiation and are paramagnetic, thus they are getting increased attention due to facilitated imaging with MRI, enabling real-time infusion and tumor coverage monitoring [163]. In addition, ${ }^{166} \mathrm{Ho}$ microspheres scout dose is used for treatment planning, enabling more accurate prediction of intra- and extrahepatic distribution of radiation activity. The differences between the RE particles are summarized in the Table 4 .

Although recent RCT failed to demonstrate the OS benefit of combination of RE with systemic chemotherapy compared with systemic chemotherapy alone in the firstline CLM treatment setting [164], subset of the patients with right-sided CRC showed OS advantage from this combinational treatment [165]. This is very important as the patients with right-sided CRC have less treatment options available than the left-sided CRC. Further investigation is of course needed to further assess this preliminary finding prior to its acceptance as a standard of care recommended in guidelines.

TACE

The objective of TACE is to generate a hypoxic/ischemic environment as well as to synergistically induce chemotherapeutic tumor destruction. TACE showed promising results for patients progressing on irinotecan-based systemic chemotherapy. TACE is usually applied to treat unresectable CLM in chemorefractory setting [72]. The data on $2^{\text {nd }}$ and $3^{\text {rd }}$ line TACE is collected from RCT [130] as well as from observational studies demonstrating wide variation of response (35-85\%) and median OS of 13.3-37 months [166], which compares favorably to other standard of care therapies for CLM.
Table 4 Comparison of ${ }^{9} \mathrm{Y}$ (resin and glass-based) and ${ }^{166} \mathrm{Ho}$ microspheres for radioembolization

\begin{tabular}{|c|c|c|c|}
\hline & SIR-Spheres $^{\mathrm{TM}}$ & Therasphere $^{\mathrm{TM}}$ & QuiremSpheres ${ }^{\mathrm{TM}}$ \\
\hline Matrix & Resin & Glass & PLLA \\
\hline Diameter & 20-6oum (mean $32 \mu \mathrm{m}$ ) & $25 \mu \mathrm{m}$ & зо нm \\
\hline Isotope & \multicolumn{2}{|c|}{ Yttrium-90 } & Holmium-166 \\
\hline $\mathrm{T}_{1 / 2}$ & \multicolumn{2}{|c|}{64.5 hours } & 26.8 hours \\
\hline Density & $1.6 \mathrm{~g} / \mathrm{cm}^{3}$ & $3.4 \mathrm{~g} / \mathrm{cm}^{3}$ & $1.4 \mathrm{~g} / \mathrm{cm}^{3}$ \\
\hline Number of spheres & $33-50 \mathrm{mln}$ & $4 \mathrm{mln}$ & $33 \mathrm{mln}$ \\
\hline Amount per dose & 900-1370 mg & $110 \mathrm{mg}$ & $600 \mathrm{mg}$ \\
\hline Activity per sphere & $50 \mathrm{~Bq}$ & $1250-2500 \mathrm{~Bq}$ & $200-400 \mathrm{~Bq}$ \\
\hline Activity per dose & 2-3 GBq & 5-15 GBq & 6-12 GBq \\
\hline Imaging & \multirow{2}{*}{\multicolumn{2}{|c|}{$\begin{array}{l}\text { Y-go PET or Bremsstrahlung SPECT/CT } \\
\text { gqm TC-MAA }\end{array}$}} & SPECT/MRI/CT \\
\hline Test dose & & & Ho-spheres/Tc-MAA \\
\hline Company & $\begin{array}{l}\text { Sirtex Medical Limited, NSW, } \\
\text { Australia }\end{array}$ & BTG, London, UK & Terumo, Nijmegen, NL \\
\hline Year of creation & 1974 & 1989 & 1994 \\
\hline CE trade mark & 2002 & 1999 & 2014 \\
\hline FDA indications & $\begin{array}{l}\text { Unresectable colorectal liver } \\
\text { metastases in combination } \\
\text { with intrahepatic floxuridine }\end{array}$ & $\begin{array}{l}\text { Humanitarian device } \\
\text { exemption for unresectable } \\
\text { HCC }\end{array}$ & $\begin{array}{l}\text { Unresectable colorectal liver } \\
\text { metastases }\end{array}$ \\
\hline Activity calculation & BSA, partition model & MIRD-based approach & $\begin{array}{l}\text { Two-compartment-based } \\
\text { dosimetry }\end{array}$ \\
\hline
\end{tabular}

DEBIRI-TACE [52] have been imposed as a novel drug-delivery vehicle allowing for higher concentrations of drugs (irinotecan) within the target tumor and lower systemic concentrations compared with conventional TACE (CTACE) [167]. cTACE is usually administered selectively/sub-selectively, whereas DEBIRI-TACE is commonly administered in the lobar fashion due to the fact, that irinotecan is a prodrug, activated by normal liver parenchyma, enabling to treat potentially occult liver lesions.

DEBIRI-TACE has been also explored for endovascular induction (neoadjuvant therapy) to target resectable CLM in PARAGON II study, with demonstrated low morbidity and 77\% major response rate on pathology [168]. Radiopaque DC Bead LUMI ${ }^{\mathrm{TM}}$ beads (BTG plc, United States), loaded with irinotecan, are the first radiopaque beads, which have been recently approved for CLM treatment, enabling to achieve much more precision for targeting, visualization of tumor coverage and defining the endpoints [169, 170]. To date, there is very limited evidence for bland embolization in CLM. 


\section{NEW IR TREATMENTS ON THE PIPELINE AND POSSIBLE MOLECULAR DRIVERS}

New IR treatments on the pipeline, including treatment of earlier stage metastatic disease, combinational LAT therapies, ablative dose RE as well as combination of immunotherapy and LAT are described in detail in Supplement 2.

Shift of the research scope in $m C R C$

At the time of OMD term introduction (2016), the main focus of IO/IR has been integration of new LATs, such as RFA, MWA, irreversible electroporation, RE and (chemo-) embolization, into mCRC treatment guidelines, improvement of techniques efficacy, identification of prognostic factors and multidisciplinarity [72]. LAT were administered for OMD patients with curative or palliative intents [72].

However, since the year 2018, main research focus has been shifted to mCRC molecular determination, identification of the patients with curable OMD based on RNA analysis, exploration of tumor-stromal interaction as well as systemic effects following LAT and immunomodulation to induce abscopal effect (non-targeted tumor response) [171]. Also, mCRC OMD treatment concept has been modified, with recommendation to administer LAT with cytoreduction and curative intent to allow for "chance for cure" [171].

Identification of curable $m C R C$ for better patient selection

It is crucial to be able to distinguish patients with potentially curable OMD from patients whose OMD is a part of a large cascade of widespread disease. This is very important as patients with new lesions following LAT of mCRC have been shown to have a poor prognosis. Prognostication strategies of CLM patients, based on RNA analysis, have been developed, indentifying three distinct CLM similarity network fusion subtypes (SNFs) with unique patterns of mRNA and miRNA expression as well as with distinct histological and genetic features and prognosis, which could help to identify OMD patients with potentially curable disease [6]. Further investigation is needed to define to what extent these concepts apply to patients undergoing LAT and, more generally, to patients with more widespread disease.

Key mutations in LAT for $m C R C$

Most explored prognostic genetic signatures include in LAT for mCRC include KRAS and $\mathrm{PI}_{3} \mathrm{~K}$ mutations. KRAS mutation was found to be an independent prognostic factor of poor outcomes following ${ }^{\circ} Y \mathrm{YE}[172,173]$ and thermal ablation of CLM [10, 11, 174]. Concordant data indicate that a minimum ablation margin of $>10 \mathrm{~mm}$ is recommended when ablating KRAS mutant CLM [174]. A minimal margin under $5 \mathrm{~mm}$ in KRAS mutant CLM carries 16.8 times the risk of local failure when compared to wild-type CLM ablated with $\mathrm{MM}$ over $10 \mathrm{~mm}$. $\mathrm{PI}_{3} \mathrm{~K}$ mutation was found to be associated with longer liver progression-free survival following ${ }^{90} Y$ RE of CLM [138].

Artificial intelligence (AI) in IR

AI has the potential to improve mCRC patient outcomes following LAT by improving patient selection and response assessment via the identification of imaging features associated with oncological outcomes. Texture analysis has demonstrated its utility in the assessment of response to RE for CLM, allowing for the detection of disease progression, on average, 3.5 months before it was visible on RECIST 1.1 [175].

Also, AI has a potential to improve catheter navigation, ablation probe placement, imaging registration and estimation of ablation margins [176]

Conclusion

In conclusion, there is expanding evidence regarding the value of LAT to improve liver and overall progression-free survival in mCRC. Evidence regarding the effect of LAT on patient OS is lacking $[14,75]$. At this time, prospective studies comparing different locoregional modalities or ablative modalities to surgery are limited and decision-making relies on limited data and a multidisciplinary decision making process. As IO techniques strive to take additional roles in the management of CLM, progress will ultimately be dependent on the ability to produce substantial prospective trial-based evidence.

\section{SUGGESTED READINGS}

Cancer Societies:

Society of Interventional Oncology (SIO): http://www.sio-central.org/

Society of Interventional Radiology (SIR): https:/|www.sirweb.org/

Society of Cardiovascular and Interventional Radiology of Europe (CIRSE): https:|| www.cirse.org/

- $\quad$ European Society of Oncologic Imaging (ESOI): http://www.esoi-society.org/

- European Organization for Research and Treatment of Cancer (EORTC): http://www. eortc.org

European Society for Medical Oncology (ESMO): https:|/www.esmo.org/ 
Guidelines:

European Society for Medical Oncology (ESMO) consensus guidelines for the management of patients with metastatic colorectal cancer [72];

NCCN Clinical Practice Guidelines in Oncology for Colon Cancer [26];

- Image-guided tumor ablation: standardization of terminology and reporting criteria--a 10-year update [177];

- Quality Improvement Guidelines for Transarterial Chemoembolization and Embolization of Hepatic Malignancy [178];

- Radioembolization of Hepatic Malignancies: Background, Quality Improvement Guidelines, and Future Directions [179];

- Transcatheter Therapy for Hepatic Malignancy: Standardization of Terminology and Reporting Criteria Reporting Standards [180].

Publications:

Ruers, T. et al. Local Treatment of Unresectable Colorectal Liver Metastases: Results of a Randomized Phase II Trial. Journal of the National Cancer Institute 109, doi:10.1093/jnci/ djxo15 (2017).

Wasan, H. S. et al. First-line selective internal radiotherapy plus chemotherapy versus chemotherapy alone in patients with liver metastases from colorectal cancer (FOXFIRE, SIRFLOX, and FOXFIRE-Global): a combined analysis of three multicentre, randomised, phase 3 trials. The Lancet. Oncology 18, 1159-1171, doi:10.1016/s1470-2045(17)30457-6 (2017).

Pitroda, S. P. et al. Integrated molecular subtyping defines a curable oligometastatic state in colorectal liver metastasis. Nature communications 9, 1793, doi:10.1038/s41467-018-042786(2018).

Shady, W., et al., Percutaneous Microwave versus Radiofrequency Ablation of Colorectal Liver Metastases: Ablation with Clear Margins (Ao) Provides the Best Local Tumor Control.J Vasc Interv Radiol, 2018. 29(2): p. 268-275.e1.

Kurilova, I., et al., Factors Affecting Oncologic Outcomes of goY Radioembolization of Heavily Pre-Treated Patients With Colon Cancer Liver Metastases. Clin Colorectal Cancer, 2019. 18(1): p. 8-18.

\section{REFERENCES}

1. National Cancer Institute Surveillance Epidemiology and End Results Program. Cancer stat facts: colon and rectum cancer. https:||seer. cancer.gov/statfacts/html/colorect.html. Accessed May 17, 2018.

2. Siegel RL, Miller KD, Jemal A. Cancer statistics, 2018. CA Cancer J Clin. 2018;68(1):7-30.

3. Ghiringhelli F, et al. Epidemiology and prognosis of synchronous and metachronous colon cancer metastases: a French population-based study. Dig Liver Dis. 2014;46(9):854-8.

4. Tarraga Lopez PJ, Albero JS, Rodriguez-Montes JA. Primary and secondary prevention of colorectal cancer. Clin Med Insights Gastroenterol. 2014:7:33-

5. Lee MS, Menter DG, Kopetz S. Right versus left colon cancer biology: integrating the consensus molecular subtypes. J Natl Compr Canc Netw. 2017;15(3):411-9.

6. Pitroda SP, et al. Integrated molecular subtyping defines a curable oligometastatic state in colorectal liver metastasis. Nat Commun. 2018;9(1):1793.

7. Pollock CB, et al. Oncogenic K-RAS is required to maintain changes in cytoskeletal organization, adhesion, and motility in colon cancer cells. Cancer Res. 2005;65(4):1244-50.

8. Tan C, Du X. KRAS mutation testing in metastatic colorectal cancer. World J Gastroenterol. 2012;18(37):5171-80.

9. Brudvik KW, et al. RAS mutation predicts positive resection margins and narrower resection margins in patients undergoing resection of colorectal liver metastases. Ann Surg Oncol. 2016;23 (8):2635-43.

10. Odisio BC, et al. Local tumour progression after percutaneous ablation of colorectal liver metastases according to RAS mutation status. Br ]
Surg. 2017;104(6):760-8.

11. Shady W, et al. Kras mutation is a marker of worse oncologic outcomes after percutaneous radiofrequency ablation of colorectal liver metastases. Oncotarget. 2017;8(39):66117-27.

12. Clarke CN, Kopetz ES. BRAF mutant colorectal cancer as a distinct subset of colorectal cancer: clinical characteristics, clinical behavior, and response to targeted therapies. J Gastrointest Oncol. 2015;6(6):660-7.

13. Nojadeh JN, Behrouz Sharif S, Sakhinia E. Microsatellite instability in colorectal cancer EXCLI J. 2018;17:159-68.

14. Ruers T, et al. Local treatment of unresectable colorectal liver metastases: results of a randomized phase II trial. J Natl Cancer Inst. 2017;109(9):djxo15.

15. Creasy JM, et al. Actual 10-year survival after hepatic resection of colorectal liver metastases: what factors preclude cure? Surgery. 2018;163(6):1238-44.

16. Fong $\mathrm{Y}$,et al. Clinical score for predicting recurrence after hepatic resection for metastatic colorectal cancer: analysis of 1001 consecutive cases. Ann Surg. 1999;230(3):309-18 discussion 318-21.

17. Shady W, et al. Percutaneous microwave versus radiofrequency ablation of colorectal liver metastases: ablation with clear margins (Ao) provides the best local tumor control. J Vasc Interv Radiol. 2018;29(2):268-275.e1.

18. Shady W, et al. Percutaneous radiofrequency ablation of colorectal cancer liver metastases: factors affecting outcomes-a 10-year experience at a single center. Radiology. 2016;278(2):601-11.

19. Sotirchos VS, et al. Colorectal cancer liver metastases: biopsy of the ablation zone and margins can be used to predict oncologic outcome. Radiology. 2016;280(3):949-59. 
20. Kurilova I, et al. Factors affecting oncologic outcomes of goY radioembolization of heavily pretreated patients with colon cancer liver metastases. Clin Colorectal Cancer. 2019;18(1):8-18.

21. Bipat S, et al. Colorectal liver metastases: CT, MR imaging, and PET for diagnosis-meta-analysis. Radiology. 2005;237(1):123-31.

22. Floriani I, et al. Performance of imaging modalities in diagnosis of liver metastases from colorectal cancer: a systematic review and meta-analysis. J Magn Reson Imaging. 2010;31(1):19-31.

23. Kulemann V, et al. Preoperative detection of colorectal liver metastases in fatty liver: MDCT or MRI? Eur J Radiol. 2011;79(2):e1-6.

24. Yoo HJ, Lee JS, Lee JM. Integrated whole body MR PET: where are we? Korean | Radiol. 2015;16(1):32-49.

25. Dietrich CF, et al. Assessment of metastatic liver disease in patients with primary extrahepatic tumors by contrast-enhanced sonography versus CT and MRI. World J Gastroenterol. 2006;12(11):1699-

26. Benson AB 3rd, et al. Colon cancer, version 1.2017, NCCN clinical practice guidelines in oncology. J Natl Compr Canc Netw. 2017;15(3):370-98.

27. Cameron J, Andrew C. Current surgical therapy. E-book 12 ed. Philadelphia: Elsevier Health Sciences 2016.

28. Adam R, Vinet E. Regional treatment of metastasis: surgery of colorectal liver metastases. Ann Oncol. 2004;15(Suppl 4):ivio3-6.

29. Parkin DM, et al. Global cancer statistics, 2002. CA CanCancer J Clin. 2005;55(2):74-108

30. Kemeny NE, et al. Updated long-term survival for patients with metastatic colorectal cancer treated with liver resection followed by hepatic arterial infusion and systemic chemotherapy. J Surg Oncol. 2016;113(5):477-84.

31. Andre T, et al. Oxaliplatin, fluorouracil, and leucovorin as adjuvant treatment for colon cancer. N Engl J Med. 2004;350(23):2343-51.

32. Kemeny $\mathrm{N}$, et al. Hepatic arterial infusion of chemotherapy after resection of hepatic metastases from colorectal cancer. N Engl J Med. 1999;341(27):2039-48

33. Kemeny MM, et al. Combined-modality treatment for resectable metastatic colorectal carcinoma to the liver: surgical resection of hepatic metastases in combination with continuous infusion of chemotherapy-an intergroup study. J Clin Oncol. 2002;20(6):1499-505.

34. Lygidakis NJ, et al. Metastatic liver disease of colorectal origin: the value of locoregional immunochemotherapy combined with systemic chemotherapy following liver resection. Results of a prospective randomized study. Hepatogastroenterology. 2001;48(42):1685-91.

35. Lorenz M, et al. Randomized trial of surgery versus surgery followed by adjuvant hepatic arterial infusion with 5 -fluorouracil and folinic acid for liver metastases of colorectal cancer. German Cooperative on Liver Metastases (Arbeitsgruppe Lebermetastasen) Ann Surg 1998:228(6):756-62.

36. Kemeny $\mathrm{N}$, et al. Hepatic arterial infusion of floxuridine and dexamethasone plus high-dose Mitomycin C for patients with unresectable hepatic metastases from colorectal carcinoma. Surg Oncol. 2005;91(2):97-101.

37. Ducreux M, et al. Hepatic arterial oxaliplatin infusion plus intravenous chemotherapy in colorectal cancer with inoperable hepatic metastases: a trial of the gastrointestinal group of the Federation Nationale des Centres de Lutte Contre le Cancer. J Clin Oncol. 2005;23(22):4881-7.

38. Chen Y, et al. Hepatic arterial infusion with irinotecan, oxaliplatin, and floxuridine plus systemic chemotherapy as first-line treatment of unresectable liver metastases from colorectal cancer. Onkologie. 2012;35(9):480-4.

39. Nordlinger B, et al. Perioperative chemotherapy with FOLFOX 4 and surgery versus surgery alone for resectable liver metastases from colorectal cance (EORTC Intergroup trial 40983): a randomized controlled trial. Lancet. 2008;371(9617):1007-16.

40. Beppu T, et al. FOLFOX enables high resectability and excellent prognosis for initially unresectable colorectal liver metastases. Anticancer Res. 2010;30(3):1015-20

41. Uetake $\mathrm{H}$, et al. A multicenter phase II trial of mFOLFOX6 plus bevacizumab to treat liver-only metastases of colorectal cancer that are unsuitable for upfront resection (TRICCo808). Ann Surg Oncol, 2015;22(3):908-15.

42. Primrose J, et al. Systemic chemotherapy with or without cetuximab in patients with resectable colorectal liver metastasis: the New EPOC randomised controlled trial. Lancet Oncol. 2014;15(6):601-11.

43. Van Cutsem E, et al. Cetuximab and chemotherapy as initial treatment for metastatic colorectal cancer. N Engl J Med. 2009;36o(14):1408-17.

44. Bokemeyer C, et al. Fluorouracil, leucovorin, and oxaliplatin with and without cetuximab in the first-line treatment of metastatic colorectal cancer. JClin Oncol. 2009;27(5):663-71.

45. Falcone A, et al. Phase III trial of infusional fluorouracil, leucovorin, oxaliplatin, and irinotecan (FOLFOXIRI) compared with infusional fluorouracil, leucovorin, and irinotecan (FOLFIRI) as first-line treatment for metastatic colorectal cancer: the Gruppo Oncologico Nord Ovest. J Clin Oncol. 2007;25(13):1670-6.

46. Masi G, et al. Long-term outcome of initially unresectable metastatic colorectal cancer patients treated with 5-fluorouraci//leucovorin, oxaliplatin, and irinotecan (FOLFOXIRI) followed by radical surgery of metastases. Ann Surg. 2009;249(3):420-5. 47. Cremolini C, et al. Efficacy of FOLFOXIRI plus bevacizumab in liver-limited metastatic colorectal cancer: a pooled analysis of clinical studies by Gruppo Oncologico del Nord Ovest. Eur J Cancer. 2017;73:74-84.

48. Tomasello $\mathrm{G}$, et al. FOLFOXIRI plus bevacizumab as conversion therapy for patients with initially unresectable metastatic colorectal cancer: a systematic review and pooled analysis. JAMA Oncol. 2017:3(7):e170278

49. D'Angelica MI, et al. Phase II trial of hepatic artery infusional and systemic chemotherapy for patients with unresectable hepatic metastases from colorectal cancer: conversion to resection and long-term outcomes. Ann Surg. 2015;261(2):353-60.

50. Pak LM, et al. Prospective phase II trial of combination hepatic artery infusion and systemic chemotherapy for unresectable colorectal liver metastases: long term results and curative potential. J Surg Oncol. 2018;117(4):634-43.

51. Shah JL, et al. Neoadjuvant transarterial radiation lobectomy for colorectal hepatic metastases: a small cohort analysis on safety, efficacy, and radiopathologic correlation. J Gastrointest Oncol. 2017;8(3):E43-e51.

52. Jones RP, et al. Neoadjuvant treatment of colorectal liver metastases (CRLM) with drug eluting beads trans-arterial chemoembolization (DEBIRI-TACE): a multi-institute phase II study in resectable metastases. J Clin Oncol. 2012;30(15_suppl):3613.

53. Livraghi T, et al. Percutaneous radiofrequency ablation of liver metastases in potential candidates for resection: the "test-of time approach". Cancer. 2003;97(12):3027-35

54 Tournigand C, et al. FOLFIRI followed by FOLFOX6 or the reverse sequence in advanced colorectal 
cancer: a randomized GERCOR study. J Clin Oncol. 2004;22(2):229-37.

55. Venook AP, et al. Effect of first-line chemotherapy combined with cetuximab or bevacizumab on overall survival in patients with KRAS wild-type advanced or metastatic colorectal cancer: a randomized clinical trial. JAMA. 2017;317(23):2392401.

56. Venook AP, Niedzwiecki D, Innocenti F, et al. Impact of primary (1_) tumor location on overall survival (OS) and progression- free survival (PFS) in patients (pts) with metastatic colorectal cancer (mCRC): analysis of CALGB/SWOG 80405 (alliance). JClin Oncol. 2016; 34, 2016 (suppl; abstr 3504).

57. Hecht JR, et al. A randomized phase IIIB trial of chemotherapy, bevacizumab, and panitumumab compared with chemotherapy and bevacizumab alone for metastatic colorectal cancer. J Clin Oncol. 2009;27(5):672-80.

58. Tol J, et al. A randomised phase III study on capecitabine, oxaliplatin and bevacizumab with or without cetuximab in firstline advanced colorectal cancer, the CAIRO2 study of the Dutch Colorectal Cancer Group (DCCG). An interim analysis of toxicity. Ann Oncol. 2008;19(4):734-8.

59. Douillard JY, et al. Randomized, phase III tria of panitumumab with infusional fluorouracil, leucovorin, and oxaliplatin (FOLFOX4) versu FOLFOX $4_{4}$ alone as first-line treatment in patients with previously untreated metastatic colorectal cancer: the PRIME study. J Clin Oncol. 2010;28(31):4697-705.

6o. Peeters M, et al. Randomized phase III study of panitumumab with fluorouracil, leucovorin, and irinotecan (FOLFIRI) compared with FOLFIRI alone as second-line treatment in patients with metastatic colorectal cancer. J Clin Oncol. 2010;28(31):4706-13.
61. Yamada Y, et al. Leucovorin, fluorouracil, and oxaliplatin plus bevacizumab versus $S-1$ and oxaliplatin plus bevacizumab in patients with metastatic colorectal cancer (SOFT): an open-label, non-inferiority, randomised phase 3 trial. Lancet Oncol. 2013;14(13):1278-86

62. Muro K, et al. Irinotecan plus S-1 (IRIS) versus fluorouracil and folinic acid plus irinotecan (FOLFIRI) as second-line chemotherapy for metastatic colorectal cancer: a randomized phase $2 / 3$ non-inferiority study (FIRIS study). Lancet Oncol. 2010;11(9):853-60.

63. Van Cutsem E, et al. Aflibercept plus FOLFIRI vs. placebo plus FOLFIRI in second-line metastatic colorectal cancer: a post hoc analysis of survival from the phase III VELOUR study subsequent to exclusion of patients who had recurrence during or within 6 months of completing adjuvant oxaliplatin-based therapy. Target Oncol. 2016;11(3):383-400.

64. Folprecht $\mathrm{G}$, et al. Oxaliplatin and $5-\mathrm{FU} /$ folinic acid (modified FOLFOX6) with or without aflibercept in first-line treatment of patients with metastatic colorectal cancer: the AFFIRM study. Ann Oncol. 2016;27(7):1273-9.

65. Tabernero J, et al. Ramucirumab versus placebo in combination with second-line FOLFIRI in patients with metastatic colorectal carcinoma that progressed during or after first-line therapy with bevacizumab, oxaliplatin, and a fluoropyrimidine (RAISE): a randomised, double-blind, multicentre, phase 3 study. Lancet Oncol. 2015;16(5):499-508.

66. Grothey A, et al. Regorafenib monotherapy for previously treated metastatic colorectal cancer (CORRECT): an international, multicentre, randomised, placebo-controlled, phase 3 trial. Lancet. 2013;381(9863):303-12.

67. Mayer RJ, et al. Randomized trial of TAS-102 for refractory metastatic colorectal cancer. N Engl Med. 2015;372(20):1909-19.

68. Cercek A, et al. Response rates of hepatic arteria infusion pump therapy in patients with metastatic colorectal cancer liver metastases refractory to all standard chemotherapies. J Surg Oncol. 2016;114(6):655-63.

69. Le DT, et al. PD-1 blockade in tumors with mismatchrepair deficiency. N Engl J Med. 2015;372(26):2509-20.

7o. Le DT, Uram J, Wang H, et al. Programmed death-1 blockade in mismatch repair deficient colorectal cancer (abstract). J Clin Oncol. 2016; 34, 2016 (suppl; abstr 103).

71. Overman MJ, Kopetz S, McDermott RS, et al. Nivolumab \pm ipilimumab in treatment (tx) of patients (pts) with metastatic colorectal cancer (mCRC) with and without high microsatellite instability (MSI-H): CheckMate-142 interim results (abstract). J Clin Oncol. 2016; 34, 2016 (suppl; abstr 3501).

72. Van Cutsem E, et al. ESMO consensus guidelines for the management of patients with metastatic colorectal cancer. Ann Oncol. 2016;27(8):1386-422.

73. Maher B, et al. The management of colorectal liver metastases. Clin Radiol. 2017;72(8):617-25.

74. Gillams A, et al. Thermal ablation of colorectal live metastases: a position paper by an international panel of ablation experts, The Interventional Oncology Sans Frontieres meeting 2013. Eur Radiol. 2015;25(12):3438-54.

75. Wasan HS, et al. First-line selective internal radiotherapy plus chemotherapy versus chemotherapy alone in patients with liver metastases from colorectal cancer (FOXFIRE, SIRFLOX, and FOXFIRE-Global): a combined analysis of three multicentre, randomised, phase 3 trials. Lancet Oncol. 2017;18(9):1159-71.

76. Yamakado K, et al. Radiofrequency ablation combined with hepaticarterialchemoembolization using degradable starch microsphere mixed with Mitomycin C for the treatment of liver metastasis from colorectal cancer: a prospective multicenter study. Cardiovasc Intervent Radiol. 2017;40(4):56o7.

77. Wu ZB, et al. Percutaneous microwave ablation combined with synchronous transcatheter arterial chemoembolization for the treatment of colorectal liver metastases: results from a follow-up cohort. Onco Targets Ther. 2016;9:3783-9.

78. Vogl TJ, et al. Survival of patients with nonresectable, chemotherapy-resistant colorectal cancer liver metastases undergoing conventional lipiodol-based transarterial chemoembolization (CTACE) palliatively versus neoadjuvantly prior to percutaneous thermal ablation. Eur J Radiol. 2018;102:138-45.

79. Ishikawa T, et al. Multiple liver metastases due to sigmoid colon cancer successfully treated by degradable starch microspheres (DSM)-TAE, radiofrequency ablation therapy, and Uzel/UFT. Gan To Kagaku Ryoho. 2010;37(2):335-8.

8o. Fong $\mathrm{ZV}$, et al. Combined hepatic arterial embolization and hepatic ablation for unresectable colorectal metastases to the liver. Am Surg. 2012;78(11):1243-8

81. Wang DS, Louie JD, Sze DY. Intra-arterial therapies for metastatic colorectal cancer. Semin Intervent Radiol. 2013;30(1):12-20.

82. Gillams AR, Lees WR. Radio-frequency ablation of colorectal liver metastases in 167 patients. Eur Radiol. 2004;14(12)::2261-7.

83. Siperstein AE, et al. Survival after radiofrequency ablation of colorectal liver metastases: 10year experience. Ann Surg. 2007;246(4):559-65 discussion 565-7.

84. Veltri A, et al. Radiofrequency ablation of colorectal 
liver metastases: small size favorably predicts technique effectiveness and survival. Cardiovasc Intervent Radiol. 2008;31(5):948-56.

85. Gillams AR, Lees WR. Five-year survival in 309 patients with colorectal liver metastases treated with radiofrequency ablation. Eur Radiol. 2009;19(5):1206-13.

86. Sofocleous CT, et al. CT-guided radiofrequency ablation as a salvage treatment of colorectal cancer hepatic metastases developing after hepatectomy. J Vasc Interv Radiol. 2011;22(6):755-61.

87. Solbiati L, et al. Small liver colorectal metastases treated with percutaneous radiofrequency ablation: local response rate and long-term survival with up to 10-year follow-up. Radiology 2012;265(3)::958-68.

88. Shibata T, et al. Microwave coagulation therapy for multiple hepatic metastases from colorectal carcinoma. Cancer. 2000;89(2):276-84.

89. Tanaka K, et al. Outcome after hepatic resection versus combined resection and microwave ablation for multiple bilobar colorectal metastases to the liver. Surgery. 2006;139(2):263-73.

go. Liang P, et al. Prognostic factors for percutaneous microwave coagulation therapy of hepatic metastases. AJR Am J Roentgenol. 2003;181(5):131925

91. Shyn PB, et al. Percutaneous imaging-guided cryoablation of liver tumors: predicting local progression on 24-hour MRI. AJR Am J Roentgenol. 2014;203(2):W181-91.

92. Vogl TJ, et al. Thermal ablation of liver metastase from colorectal cancer: radiofrequency, microwave and laser ablation therapies. Radiol Med. 2014;119(7):451-61.

93. Sartori S, et al. Laser ablation of liver tumors an ancillary technique, or an alternative to radiofrequency and microwave? World J Radiol.
2017;9(3):91-6.

94. Sartori S, Tombesi P, Di Vece F. Thermal ablation in colorectal liver metastases: lack of evidence or lack of capability to prove the evidence? World J Gastroenterol. 2016;22(13):3511-5.

95. Park MY, et al. Preliminary experience using high intensity focused ultrasound for treating liver metastasis from colon and stomach cancer. Int $\mathrm{J}$ Hyperthermia. 2009;25(3):180-8.

96. Lyu T, et al. Irreversible electroporation in primary and metastatic hepatic malignancies: a review. Medicine (Baltimore). 2017;96(17):e6386.

97. Scheffer $\mathrm{HJ}$, et al. Irreversible electroporation for colorectal liver metastases. Tech Vasc Interv Radiol. 2015;:18(3):159-69.

98. Schoellhammer HF, et al. Colorectal liver metastases: making the unresectable resectable using irreversible electroporation for microscopic positive margins-a case report. BMC Cancer. 2015;15:271.

99. Weng M, et al. Radiofrequency ablation versus resection for colorectal cancer liver metastases: a meta-analysis. PLoS ONE. 2012;7(9):e45493.

100. Mulier $S$, et al. Radiofrequency ablation versus resection for resectable colorectal liver metastases: time for a randomized trial? An update. Dig Surg. 2008;25(6):445-6o.

101. Pathak S, et al. Ablative therapies for colorectal liver metastases: a systematic review. Colorectal Dis. 2011;13(9):e252-65.

102. Ruers $\mathrm{T}$, et al. Radiofrequency ablation combined with systemic treatment versus systemic treatment alone in patients with non-resectable colorectal liver metastases: a randomized EORTC Intergroup phase II study (EORTC 40004). Ann Oncol. 2012;23(10):2619-26.

103. Petre EN, et al. Treatment of pulmonary colorectal metastases by radiofrequency ablation. Clin
Colorectal Cancer. 2013;12(1):37-44.

104. Mouli SK, et al. The role of percutaneous imageguided thermal ablation for the treatment of pulmonary malignancies. AJR Am J Roentgenol. 2017;209(4):740-51.

105. Bruix J, Sherman M. Management of hepatocellular carcinoma. Hepatology. 2005;42(5):1208-36. Kudo M, Okanoue T. Management of hepatocellular carcinoma in Japan: consensus-based clinical practice manual proposed by the Japan Society of Hepatology. Oncology. 2007;72(Suppl 1):2-15.

106. Kudo M, Okanoue T. Management of hepatocellular carcinoma in Japan: consensus-based clinical practice manual proposed by the Japan Society of Hepatology. Oncology. 2007;72(Suppl1):2-15.

107. Omata M, et al. Asian Pacific Association for the Study of the Liver consensus recommendations on hepatocellular carcinoma. Hepatol Int 2010;4(2):439-74

108. Ayav A, et al. Radiofrequency ablation of unresectable liver tumors: factors associated with incomplete ablation or local recurrence. Am J Surg. 2010;200(4):435-9.

109. Amersi FF, et al Long-term survival after tadiofrequency ablation of complex unresectable liver tumors. Arch Surg. 2006;141(6):581-7 discussion $587-8$.

110. Veenendaal LM, Borel Rinkes IH, van Hillegersberg R. Multipolar radiofrequency ablation of large hepatic metastases of endocrine tumours. Eur Gastroenterol Hepatol. 2006;18(1):89-92.

111. Hur H, et al. Comparative study of resection and radiofrequency ablation in the treatment of solitary colorectal liver metastases. Am J Surg. 2009;197(6):728-36.

112. Kim YS, et al. Intrahepatic recurrence after percutaneous radiofrequency ablation of hepatocellular carcinoma: analysis of the pattern and risk factors. Eur J Radiol. 2006;59(3):432-41.

113. Mulier $S$, et al. Local recurrence after hepatic radiofrequency coagulation: multivariate metaanalysis and review of contributing factors. Ann Surg. 2005;242(2):158-71.

114. Lee WS, et al. Clinical outcomes of hepatic resection and radiofrequency ablation in patients with solitary colorectal liver metastasis. J Clin Gastroenterol. 2008;42(8):945-9.

115. Van Tilborg AA, et al. Long-term results of radiofrequency ablation for unresectable colorectal liver metastases: a potentially curative intervention. Br J Radiol. 2011;84(1002):556-65.

116. Berber E, Pelley R, Siperstein AE. Predictors of survival after radiofrequency thermal ablation of colorectal cancer metastases to the liver: a prospective study. J Clin Oncol. 2005;23(7):1358-64.

117. Abdalla EK, et al. Recurrence and outcomes following hepatic resection, radiofrequency ablation, and combined resection/ablation for colorectal liver metastases. Ann Surg. 2004;239(6):818-25 discussion 825-7.

118. Wang $\mathrm{X}$, et al. Margin size is an independent predictor of local tumor progression after ablation of colon cancer liver metastases. Cardiovasc Intervent Radiol. 2013;36(1):166-75.

119. Ryan ER, et al. Split-dose technique for FDG PET/ CT-guided percutaneous ablation: a method to facilitate lesion targeting and to provide immediate assessment of treatment effectiveness. Radiology. 2013;268(1):288-95.

120. Sofocleous CT, et al. Histopathologic and immunohistochemical features of tissue adherent to multitined electrodes after RF ablation of liver malignancies can help predict local tumor progression: initial results. Radiology. 2008;249(1):364-74.

121. Puijk RS, et al. Colorectal liver metastases: surgery 
versus thermal ablation (COLLISION)-a phase III single-blind prospective randomized controlled trial. BMC Cancer. 2018;18(1):821.

122. Correa-Gallego C, et al. A retrospective comparison of microwave ablation vs. radiofrequency ablation for colorectal cancer hepatic metastases. Ann Surg Oncol. 2014;21(13): $4278-83$.

123. Qian GJ, et al. Efficacy of microwave versus radiofrequency ablation for treatment of small hepatocellular carcinoma: experimental and clinical studies. Eur Radiol. 2012;22(9):1983-9o.

124. Di Vece $F$, et al. Coagulation areas produced by cool-tip radiofrequency ablation and microwave ablation using a device to decrease back-heating effects: a prospective pilot study. Cardiovasc Intervent Radiol. 2014;37(3):723-9.

125. Cavagnaro $\mathrm{M}$, et al. A minimally invasive antenna for microwave ablation therapies: design, performances, and experimental assessment. IEEE Trans Biomed Eng. 2011;58(4):949-59.

126. Sag AA, Selcukbiricik F, Mandel NM. Evidencebased medical oncology and interventiona radiology paradigms for liver dominant colorectal cancer metastases. World J Gastroenterol. 2016;22(11):3127-49.

127. Aliberti C, et al. Trans-arterial chemoembolization of metastatic colorectal carcinoma to the liver adopting DC Bead(R), drug eluting bead loaded with irinotecan: results of a phase II clinical study. Anticancer Res. 2011;31(12):4581-7.

128. Martin RC, et al. Hepatic intra-arterial injection of drug-eluting bead, irinotecan (DEBIRI) in unresectable colorectal liver metastases refractory to systemic chemotherapy: results of multiinstitutional study. Ann Surg Oncol. 2011;18(1):192-8.

129. Iezzi R, et al. Trans-arterial chemoembolization with irinotecan loaded drug-eluting beads (DEBIRI) and capecitabine in refractory liver prevalent colorectal metastases: a phase II single- center study. Cardiovasc Intervent Radiol. 2015;38(6):1523-

130. Fiorentini G, et al. Intra-arterial infusion of irinotecan-loaded drug-eluting beads (DEBIRI) versus intravenous therapy (FOLFIRI) for hepatic metastases from colorectal cancer: final results of a phase III study. Anticancer Res. 2012;32(4):1187-95.

131. Albert M, et al. Chemoembolization of colorectal liver metastases with cisplatin, doxorubicin, mitomycin C, ethiodol, and polyvinyl alcohol. Cancer. 2011;117(2):343-52.

132. Vogl TJ, et al. Regional chemotherapy of the lung: transpulmonary chemoembolization in malignant lung tumors. Semin Intervent Radiol. 2013;30(2):176-84.

133. Sofocleous CT, et al. Radioembolization as a salvage therapy for heavily pretreated patients with colorectal cancer liver metastases: factors that affect outcomes. Clin Colorectal Cancer. 2015;14(4):296-305.

134. Shady W, et al. Metabolic tumor volume and total lesion glycolysis on FDG-PET/CT can predict overall survival after (90)Y radioembolization of colorectal liver metastases: a comparison with SUVmax, SUVpeak, and RECIST 1.o. Eur J Radiol. 2016;85(6):1224-31.

135. Boas FE, Bodei L, Sofocleous CT. Radioembolization of colorectal liver metastases: indications, technique, and outcomes. J Nucl Med. 2017;58(Suppl 2):104s-11s

136. Sofocleous CT, et al. Phase I trial of selective internal radiation therapy for chemorefractory colorectal cancer liver metastases progressing after hepatic arterial pump and systemic chemotherapy. Clin Colorectal Cancer. 2014;:13(1):27-36.

137. Sotirchos VS, et al. Safe and successful Yttrium-9o resin microsphere radioembolization in a heavily pretreated patient with chemorefractory colorectal liver metastases after biliary stent placement above the papilla. Case Reports Hepatol. 2014;2014:921406.

138. Ziv E, et al. PI3K pathway mutations are associated with longer time to local progression after radioembolization of colorectal liver metastases. Oncotarget. 2017;8(14):23529-38.

139. Braat A, et al. Adequate SIRT activity dose is as important as adequate chemotherapy dose. Lancet Oncol. 2017;18(11): $: 636$

140. Dutton SJ, et al. FOXFIRE protocol: an open-label, randomised, phase III trial of 5 -fluorouracil, oxaliplatin and folinic acid (OxMdG) with or without interventional Selective Internal Radiation Therapy (SIRT) as first-line treatment for patients with unresectable liver-only or liverdominant metastatic colorectal cancer. BMC Cancer. 2014;14:497.

141. Gibbs P, et al. Selective Internal Radiation Therapy (SIRT) with yttrium-go resin microspheres plus standard systemic chemotherapy regimen of FOLFOX versus FOLFOX alone as first-line treatment of non-resectable liver metastases from colorectal cancer: the SIRFLOX study. BMC Cancer. 2014;14:897.

142. Khajornjiraphan N, Thu NA, Chow PK. Yttrium-go microspheres: a review of its emerging clinical indications. Liver Cancer. 2015;4(1):6-15.

143. Murray D, McEwan AJ. Radiobiology of systemic radiation therapy. Cancer Biother Radiopharm. 2007;22(1):1-23.

144. Campbell AM, Bailey IH, Burton MA. Analysis of the distribution of intra-arterial microspheres in human liver following hepatic yttrium-9o microsphere therapy. Phys Med Biol. 2000;45(4):1023-33.

145. Kennedy A, et al. Recommendations for radioembolization of hepatic malignancies using yttrium-go microsphere brachytherapy: a consensus panel report from the radioembolization brachytherapy oncology consortium. Int J Radiat Oncol Biol Phys. 2007;68(1):13-23.

146. HendliszA, etal. Phase III trial comparing protracted intravenous fluorouracil infusion alone or with yttrium-go resin microspheres radioembolization for liver-limited metastatic colorectal cancer refractory to standard chemotherapy. J Clin Oncol. 2010;28(23):3687-94.

147. Van Hazel G, et al. Randomised phase 2 trial of SIR-Spheres plus fluorouracil/leucovorin chemotherapy versus fluorouracil/ leucovorin chemotherapy alone in advanced colorectal cancer. J Surg Oncol. 2004;88(2):78-85.

148. Gray B, et al. Randomised trial of SIR-Spheres plus chemotherapy vs. chemotherapy alone for treating patients with liver metastases from primary large bowel cancer. Ann Oncol. 2001;12(12):1711-20.

149. Kennedy AS, et al. Pathologic response and microdosimetry of (90)Y microspheres in man: review of four explanted whole livers. Int J Radiat Oncol Biol Phys. 2004;60(5):1552-63.

150. Sharma RA, et al. Radioembolization of liver metastases from colorectal cancer using yttrium-9o microspheres with concomitant systemic oxaliplatin, fluorouracil, and leucovorin chemotherapy. J Clin Oncol. 2007;25(9):1099-106.

151. Vente MA, et al. Yttrium-9o microsphere radioembolization for the treatment of liver malignancies: a structured meta-analysis. Eur Radiol. 2009;19(4):951-9.

152. Cosimelli M, et al. Multi-centre phase II clinical trial of yttrium- go resin microspheres alone in unresectable, chemotherapy refractory colorectal liver metastases. Br J Cancer. 2010;103(3):324-31.

153. Kurilova I, et al. (90)Y resin microspheres radioembolization for colon cancer liver metastases using full-strength contrast material. 
Cardiovasc Intervent Radiol. 2018;41(9):1419-27.

154. Puippe G, Pfammatter T, Schaefer N. Arterial therapies of noncolorectal liver metastases. Viszeralmedizin. 2015;31(6):414-22.

155. Fan $\mathrm{KY}$,etal.Neuroendocrine tumor liver metastases treated with yttrium-9o radioembolization. Contemp Clin Trials. 2016;50:143-9.

156. Gordon AC, Salem R, Lewandowski RJ. Yttrium-go radioembolization for breast cancer liver metastases. J Vasc Interv Radiol. 2016;27(9):1316-9.

157. Bester L, et al. Radioembolisation with Yttrium-9o microspheres: an effective treatment modality for unresectable liver metastases. J Med Imaging Radiat Oncol. 2013:57(1):72-80.

158. Seidensticker R, et al. Matched-pair comparison of radioembolization plus best supportive care versus best supportive care alone for chemotherapy refractory liver-dominant colorectal metastases. Cardiovasc Intervent Radiol. 2012;35(5):1066-73.

159. Burrill J, Hafeli U, Liu DM. Advances in radioembolization- embolics and isotopes. Nucl Med Radiat Ther. 2011;2:107.

160. Piana PM, et al. Early arterial stasis during resinbased yttrium- go radioembolization: incidence and preliminary outcomes. HPB (Oxford). 2014;16(4):336-41.

161. Murthy R, et al. Yttrium go resin microspheres for the treatment of unresectable colorectal hepatic metastases after failure of multiple chemotherapy regimens: preliminary results. J Vasc Interv Radiol. 2005;16(7):937-45.

162. Chao C, et al. Effect of substituting 50\% isovue for sterile water as the delivery medium for SIRspheres: improved dose delivery and decreased incidence of stasis. Clin Nucl Med. 2017;42(3):176-

163. Prince JF, et al. Efficacy of radioembolization with (166)Homicrospheres in salvage patient with liver metastases: a phase 2 study. J Nucl Med.
2018; $599(4): 582-8$.

164. van Hazel GA, et al. SIRFLOX: randomized phase III trial comparing first-line mFOLFOX6 (plus or minus bevacizumab) versus mFOLFOX6 (plus or minus bevacizumab) plus selective internal radiation therapy in patients with metastatic colorectal cancer. J Clin Oncol. 2016;34(15):1723-31.

165. Gibbs P, et al. Effect of primary tumor side on survival outcomes in untreated patients with metastatic colorectal cancer when selective internal radiation therapy is added to chemotherapy: combined analysis of two randomized controlled studies. Clin Colorectal Cancer. 2018;17(4):e617-29.

166. Richardson Aj, Laurence JM, Lam VW. Transarterial chemoembolization with irinotecan beads in the treatment of colorectal liver metastases: systematic review. J Vasc Interv Radiol. 2013;24(8):1209-17.

167. Song JE, Kim DY. Conventional vs drug-eluting beads transarterial chemoembolization for hepatocellular carcinoma. World J Hepatol. 2017;9(18):808-14.

168. Jones RP, et al. PARAGON II-a single arm multicentre phase II study of neoadjuvant therapy using irinotecan bead in patients with resectable liver metastases from colorectal cancer. Eur J Surg Oncol. 2016;42(12):1866-72.

169. Levy EB, et al. First human experience with directly image-able iodinated embolization microbeads. Cardiovasc Intervent Radiol. 2016;39(8):1177-86.

170. Caine M, et al. Comparison of microsphere penetration with LC Bead LUMI versus other commercial microspheres. J Mech Behav Biomed Mater. 2018;78:46-55.

171. Yoshino T, et al. Pan-Asian adapted ESMO consensus guidelines for the management of patients with metastatic colorectal cancer: a JSMO-ESMO initiative endorsed by CSCO, KACO, MOS, SSO and TOS. Ann Oncol. 2018;29(1):44-7
172. Lahti SJ, et al. KRAS status as an independent prognostic factor for survival after Yttrium-go radioembolization therapy for unresectable colorectal cancer liver metastases. J Vasc Interv Radiol. 2015;26(8):1102-11.

173. Janowski E, et al. Yttrium-9o radioembolization for colorectal cancer liver metastases in KRAS wildtype and mutant patients: clinical and ccfDNA studies. Oncol Rep. 2017;37(1):57-65.

174. Calandri M, et al. Ablation of colorectal liver metastasis: interaction of ablation margins and RAS mutation profiling on local tumour progressionfree survival. Eur Radiol. 2018;28(7):2727-34

175. Reimer RP, Reimer P, Mahnken AH. Assessment of therapy response to transarterial radioembolization for liver metastases by means of post-treatment MRI-based texture analysis. Cardiovasc Intervent Radiol. 2018;41(10):1545-56.

176. Letzen B, Wang CJ, Chapiro J. The role of artificial intelligence in interventional oncology: a primer.] Vasc Interv Radiol. 2019;30(1):38-41.e1.

177. Ahmed M, et al. Image-guided tumor ablation: standardization of terminology and reporting criteria-a 10-year update. Radiology. 2014;;273(1):241-60.

178. Gaba RC, et al. Quality improvement guidelines for transarterial chemoembolization and embolization of hepatic malignancy. J Vasc Interv Radiol. 2017;28(9):1210-1223.e3.

179. Padia SA, et al. Radioembolization of hepatic malignancies: background, quality improvement guidelines, and future directions. J Vasc Interv Radiol. 2017;28(1):1-15.

180. Gaba RC, et al. Transcatheter therapy for hepatic malignancy: standardization of terminology and reporting criteria. J Vasc Interv Radiol. 2016;27(4):457-73.

181. Kemeny NE, Gonen M. Hepatic arterial infusion after liver resection. N Engl J Med. 2005;352(7):734-5.

182. Clavien PA, et al. Downstaging of hepatocellular carcinoma and liver metastases from colorectal cancer by selective intra-arterial chemotherapy. Surgery. 2002;131(4):433-42.

183. Kemeny N, et al. Phase I trial of systemic oxaliplatin combination chemotherapy with hepatic arterial infusion in patients with unresectable liver metastases from colorectal cancer. J Clin Oncol. 2005;23(22):4888-96.

184. Kemeny NE, et al. Conversion to resectability using hepatic artery infusion plus systemic chemotherapy for the treatment of unresectable liver metastases from colorectal carcinoma. J Clin Oncol. 2009;27(21):3465-71.

185. Gallagher DJ, et al. Hepatic arterial infusion plus systemic irinotecan in patients with unresectable hepatic metastases from colorectal cancer previously treated with systemic oxaliplatin: a retrospective analysis. Ann Oncol. 2007;18(12):1995-

186. Goere D, et al. Prolonged survival of initially unresectable hepatic colorectal cancer patients treated with hepatic arterial infusion of oxaliplatin followed by radical surgery of metastases. Ann Surg. 2010;251(4):686-91.

187. Levi FA, et al. Conversion to resection of liver metastases from colorectal cancer with hepatic artery infusion of combined chemotherapy and systemic cetuximab in multicenter trial OPTILIV. Ann Oncol. 2016;27(2):267-74.

188. Cercek A, et al. Floxuridine hepatic arterial infusion associated biliary toxicity is increased by concurrent administration of systemic bevacizumab. Ann Surg Oncol. 2014;21(2):479-86.

189. Xu C, et al. Radiofrequency ablation for liver metastases after transarterial chemoembolization: a systemic analysis. Asian Pac J Cancer Prev. 
2015;16(12)::101-6.

190. Bloomston M, et al. Transcatheter arterial chemoembolization with or without radiofrequency ablation in the management of patients with advanced hepatic malignancy. Am Surg. 2002;68(9):827-31.

191. Meiers C, et al. Safety and initial efficacy of radiation segmentectomy for the treatment of hepatic metastases. J Gastrointest Oncol. 2018;9(2):311-5.

192. Vouche M, et al. Unresectable solitary hepatocellular carcinoma not amenable to radiofrequency ablation: multicenter radiology pathology correlation and survival of radiation segmentectomy. Hepatology. 2014;60(1):192-201.

193. Teo JY, et al. A systematic review of contralateral liver lobe hypertrophy after unilobar selective internal radiation therapy with Ygo. HPB (Oxford). 2016;18(1):7-12.

194. Fernandez-Ros N, et al. Partial liver volume radioembolization induces hypertrophy in the spared hemiliver and no major signs of portal hypertension. HPB (Oxford). 2014;i16(3):243-9.

195. Marabelle A, et al. Starting the fight in the tumor: expert recommendations for the development of human intratumoral immunotherapy (HIT-IT) Ann Oncol. 2018;29(11):2163-74.

196. Broughton G 2nd, Janis JE, Attinger CE. Wound healing: an overview. Plast Reconstr Surg. 2006;117(7 Suppl):1-32e-S.

197. Li LY, et al. Prospective comparison of five mediators of the systemic response after highintensity focused ultrasound and targeted cryoablation for localized prostate cancer. BJU Int. 2009;104(8):1063-7.

198. Schell SR, et al. Pro- and antiinflammatory cytokine production after radiofrequency ablation of unresectable hepatic tumors. J Am Coll Surg 2002;195(6):774-81.
199. de Jong KP, et al. Serum response of hepatocyte growth factor, insulin-like growth factor-I, interleukin-6, and acute phase proteins in patients with colorectal liver metastases treated with partial hepatectomy or cryosurgery. J Hepatol. 2001;34(3):422-7.

20o. Erinjeri JP, et al. Image-guided thermal ablation of tumors increases the plasma level of interleukin-6 and interleukin-10. J Vasc Interv Radiol. 2013;24(8):1105-12.

201. Takahashi Y, et al. Immunological effect of local ablation combined with immunotherapy on solid malignancies. Chin J Cancer. 2017;36(1):49.

202. Slovak $R$, et al. Immuno-thermal ablationsboosting the anticancer immune response. J Immunother Cancer. 2017;5(1):78.

203. Lemdani K, et al. Improvement of immune response after radiofrequency ablation in colorectal cancer. Clin Oncol. 2018;36(5_suppl):102.

204. Katz SC, et al. Phase I hepatic immunotherapy for metastases study of intra-arterial chimeric antigen receptor-modified T-cell therapy for CEA liver metastases. Clin Cancer Res. 2015;21(14):3149-59.

205. Fong $\mathrm{Y}$, et al. A herpes oncolytic virus can be delivered via the vasculature to produce biologic changes in human colorectal cancer. Mol Ther 2009;1(2):389-94

206. Mehta A, Oklu R, Sheth RA. Thermal ablative therapies and immune checkpoint modulation: can locoregional approaches effect a systemic response? Gastroenterol Res Pract. 2016;2016:9251375.

207. Haen SP, et al. More than just tumor destruction: immunomodulation by thermal ablation of cancer. Clin Dev Immunol. 2011;2011:160250.

208. Fagnoni FF, et al. Combination of radiofrequency ablation and immunotherapy. Front Biosci. 2008;13:369-81.
209. den Brok MH, et al. Saponin-based adjuvants create a highly effective anti-tumor vaccine when combined with in situ tumor destruction. Vaccine. 2012;30(4):737-44.

210. den Brok MH, et al. Saponin-based adjuvants induce crosspresentation in dendritic cells by intracellular lipid body formation. Nat Commun. 2016;7:13324.

211. van den Bijgaart RJ, et al. Thermal and mechanical high-intensity focused ultrasound: perspectives on tumor ablation, immune effects and combination strategies. Cancer Immunol Immunother. 2017;66(2):247-58.

212. Nierkens $S$, et al. In vivo colocalization of antigen and CpG [corrected] within dendritic cells is associated with the efficacy of cancer immunotherapy. Cancer Res. 2008;68(13):5390-6.

213. Grivennikov SI, Greten FR, Karin M. Immunity, inflammation, and cancer. Cell. 2010;140(6):883-99.

214. Trikha $\mathrm{M}$, et al. Targeted anti-interleukin-6 monoclonal antibody therapy for cancer: a review of the rationale and clinical evidence. Clin Cancer Res. 2003;9(13):4653-65. 


\section{SUPPLEMENTARY MATERIAL}

Supplement 1 HAI therapy in the adjuvant, neoadjuvant and metastatic settings.

HAI therapy as adjuvant after liver metastatectomy

The MSKCC trial enrolled 156 patients after liver metastatectomy to receive six cycles (months) of HAI FUDR in combination with systemic chemotherapy of 5 -FU/LV or systemic FU/LV alone. 2-year OS rate was significantly higher in HAI therapy, 86 vs. 72\% $(p=0.03)$, so was liver-free recurrence rate (6o vs. 90\% $(p<0.001)$, this was confirmed in later report after a 10-year follow up [32, 181].

Another ECOG and SWOG study also demonstrated adjuvant HAI of FUDR plus systemic infusional FU was associated with a better 4 -year hepatic recurrence-free survival ( 67 vs. $43 \%, p=0.03$ ) and 4 -year overall recurrence-free survival ( 46 vs. $25 \%, p=0.04$ ) compared with surgery alone [33].

This clinical benefit was also seen in Lygidakis' study and a German study $[34,35]$.

HAI therapy alone or in combination with systemic chemotherapy as conversion therapy Conversion therapy options are as follows:

- $\quad$ HAI therapy alone [182];

- HAI with FUDR in combination with systemic oxaliplatin and irinotecan [183, 184];

- HAI with FUDR/dexamethasone in combination with systemic irinotecan [185];

- $\quad$ HAI with oxaliplatin in combination systemic 5-FU/LV [186];

- HAI with FUDR/dexamethasone in combination with systemic FOLFOX, FOLFIRI $[49,50]$

- $\quad$ HAI with 5-FU/oxaliplatin/irinotecan in combination with cetuximab [187].

HAI therapy in metastatic setting

HAI with FUDR/dexamethasone can be used in combination with systemic FOLFOX, FOLFIRI [49, 50, 188].
Supplement 2 New Interventional Oncology/Radiology treatments on the pipeline

Treatment of earlier stage $m C R C$ by LAT

Treatment of earlier stage mCRC by LAT within neoadjuvant, first or second line setting are being actively explored in order to achieve better tumor response rates and delay introduction of systemic chemotherapy to preclude systemic toxicity at early stages of $\operatorname{mCRC}[51,52,75]$.

Combinational LAT therapies

New combinational LAT treatments are being actively explored in terms of ablative and endovascular therapies combination in order to achieve synergistic effect to treat CLMs [76-8o, 189, 190].

Ablative dose radioembolization

${ }^{90} \mathrm{Y}$ radiation segmentectomy [191] and ${ }^{\circ} \mathrm{Y}$ radiation lobectomy [51] concepts are being actively explored in order to treat CLM patients with limited treatment options, such as those ineligible for liver resection or thermal ablation due to tumor size, insufficient ablation margins or unfavorable tumor location.

Radiation segmentectomy enables selective delivery of very high radiation doses ( $>190$ Gy) [192] to eradicate the tumor-containing liver segments while sparing uninvolved liver parenchyma $[193,194] .{ }^{\circ} Y$ radiation lobectomy additionally induces contralateral liver lobe hypertrophy, enabling portal vein embolization (PVE) - refractory patients treatment or providing a viable alternative to PVE, at the same time enabling to observe disease biology within the "test of time" concept while sufficient hypertrophy is achieved $[51,191]$.

LAT in combination with immunotherapy: role in tumor priming and selective immunotherapy delivery

LAT has an important role in tumor priming, inducing a systemic effect by local/ locoregional tumor targeting by means of heat-, cold-based and mechanical ablation techniques as well as endovascular therapies. Thus, besides having enestic effect (response of targeted lesion), it is possible to achieve an anenestic effect (response in non-targeted lesion) [195]. This is achieved due to the fact, that LAT create an "internal wound", which initiates a stereotypical wound healing immune response and cytokine release [196-199]. However, LAT can both enhance and inhibit the immune response, with subsequent inhibition or promotion of the tumor growth. Modulation of the immune response to LAT is highly challenging and is an active field of research. 
LAT monotherapy is insufficient to induce a clinically significant anenestic effect. The combination of LAT with systemic immunotherapy however has the potential to improve response rates of both immunogenic and non-immunogenic tumors by enhancing tumor immune infiltration and converting "cold" tumors into "hot" tumors [200-212]. Immunomodulatory effects of various LAT therapies are currently being explored, however, it is still to be defined which LAT is the most effective in enhancing immune response [200-203, 206-208, 211].

Another point of potential synergy between immunotherapy and IR would be selective immunotherapy delivery to the target lesion in terms of HIT-IT (human intra-tumoral immunotherapy, e.g. cutaneous or visceral intra-tumoral T-VEC administration) and LOC-IT (locoregional immunotherapy, intra-arterial). The concepts of HIT-IT and LOC-IT are currently being actively explored in advanced metastatic disease. Recent research demonstrated that T-VEC can be delivered safely into the human bloodstream to produce selective infection of CLM and replicate in cancer cells with no signs of viral reactivation elsewhere [205]. Intra-hepatic delivery of anti-CEA CAR-T cells through percutaneous hepatic artery infusions has also been explored [204]. While the application of targeted immunotherapy delivery in neoadjuvant setting is much less explored, it remains very promising in terms of achieving high local tumor control rates with a with the aims of having tumors that do not progress/recur following surgical resection or LAT.

Furthermore, ablation can also induce high systemic levels of IL-6, directly supporting cancer cell survival [213]. This can lead to persistence of viable tumor cells in the ablation zone margin and promote disease progression in distant tumor sites. Thus, IL-6 might also be targeted to enhance the treatment efficacy of thermal ablation [214]. 
Factors Associated with

Local Tumor Control and

Complications after Thermal

Ablation for Colorectal

Cancer Liver Metastases:

a 15-year Retrospective

Cohort Study

Kurilova I., Bendet A., Beets-Tan R.G.H., Petre N.E., Boas F.E., Kaye E., Gonen M., Covey A., Brody L.A., Brown K.T., Kemeny N.E., Yarmohammadi H., Ziv E., D’ Angelica M.I, Kingham P.T., Solomon S.B., Sofocleous C.T. 


\section{ABSTRACT}

Purpose

To identify risk factors associated with local tumor progression-free survival (LTPFS) and complications after colorectal liver metastases (CLM) thermal ablation (TA).

Methods

This retrospective analysis included 286 patients with 415 CLMs undergoing TA (RFA, MWA) in 378 procedures from 1/2003 to 7/2017. Prior hepatic artery infusion (HAI), bevacizumab, pre-existing biliary dilatation, ablation modality, minimal ablation margin (MM), prior hepatectomy, CLM number and size were analyzed as factors impacting complications and LTPFS. Statistical analysis included Kaplan-Meier method, Cox proportional hazards model, competing risk analysis, univariate/multivariate logistic/exact logistic regressions and Fisher's exact test. Complications were reported according to modified SIR guidelines.

Results

Median follow-up was 31 months. There was no LTP for MM >10mm. Smaller tumor size, increased MM and prior hepatectomy correlated with longer LTPFS. Major complication rate was $28 / 378$ ( $7 \%$ ). There were no biliary complications in HAI-naïve patients, vs $11 \%$ in HAI patients $(\mathrm{p}<0.001)$, of which $7 \%$ were major. Biliary complications predictors in HAI patients included: biliary dilatation, bevacizumab and $M M>10 \mathrm{~mm}$. In HAI patients ablation with 6-10mm and $>10 \mathrm{~mm}$ MM resulted in major biliary complication rates of $4 \%$ and $21 \%$ ( $\mathrm{p}=0.0011)$, with corresponding LTP rates of $24 \%$ and $\mathrm{o} \%$, $(\mathrm{p}=0.0033)$. In HAI-naïve patients LTP rates for $6-10 \mathrm{~mm}$ and $>10 \mathrm{~mm}$ MM were $27 \%$ and $0 \%$, respectively.

\section{Conclusions}

No LTP was seen for MM $>10 \mathrm{~mm}$. Biliary complications occurred only in HAI patients, especially in those with biliary dilatation, bevacizumab and $M M>10 \mathrm{~mm}$. In HAI patients MM of 6-10mm resulted in $76 \%$ local tumor control and $4 \%$ major biliary complications incidence.

\section{INTRODUCTION}

Thermal ablation (TA) is a minimally invasive treatment for selected patients with relatively small colorectal cancer liver metastases (CLM) [1-4]. When combined with standard of care chemotherapy TA (with or without liver resection) resulted in significant patient survival benefit when compared to the group treated only with chemotherapy in a randomized controlled trial [5].

The most common TA modalities - radiofrequency (RFA) and microwave ablation (MWA) - are safe and effective with similar safety profiles [6,7]. Despite overall survival results, similar to those for surgical resection [8-10], relatively high local tumor progression (LTP) rates remain an obstacle for the widespread use of thermal ablation [2,11,12].

Major complication rates of 4-33\% for RFA and 0-19\% for MWA have been reported, with pooled mortality rates of $0.15 \%$ and $0.23 \%$, respectively $[13,14]$. A wide range of biliary, vascular and other complications have been reported as a result of the thermal injury to the liver and adjacent organs, infections or deterioration of pre-existing comorbidities $[3,15,22]$. The trade-off between risks and optimal local tumor control, as well as awareness of certain steps or techniques to avoid complications while maintaining the best possible oncologic outcomes are extremely important.

CLM patients often present for thermal ablation with disease progression after systemic chemotherapy, targeted therapy, hepatic resection and in some institutions after prior hepatic artery infusion therapy (HAI)[23-28]. HAI can prolong liver progression-free and overall survival [29-32], but it has also been associated with hepatocellular injury (chemical hepatitis) and cholestatic hepatic injury (biliary strictures, chemotherapyinduced biliary sclerosis, CIBS)[33-37]. HAI-related symptomatic biliary strictures, with an incidence of 5-25\%, potentially result from direct toxicity, fibrotic encasement of the portal triad and hepatic arteritis involving the small vessels, supplying the bile ducts. Subsequent ischemia can result in ischemic biliary strictures that may be detected radiographically.

Bevacizumab exposure predisposes to hemorrhagic complications, wound healing problems, fistula formation as well as arterial and venous thromboembolism after surgery or other interventions $[26,38,39]$ Bevacizumab increased biliary complications when administered with HAI [30]. Additional possible risk factors associated with complications after TA include the type of approach (e.g. open, laparoscopic, percutaneous), number, size and location of target tumors, underlying hepatic disease 
and physicians experience [40-44].

This study was conducted to identify incidence and risk factors associated with local tumor control and complications post-TA of colorectal liver metastases (CLM). Tumor-, procedure-, imaging-related clinical parameters as well as prior treatments were analyzed as risk factors of complications and LTP. Association of complications with overal survival was also analyzed.

Our hypothesis was that prior HAI, pre-existing biliary dilatation, bevacizumab administration within 1-month of TA, prior hepatectomy, increased minimal ablation margin (MM) size [4,45,46], ablation modality, number and size of ablated tumors could impact complication rates and LTPFS following TA of CLM.

\section{MATERIALS AND METHODS}

This is an IRB-approved retrospective review of a pre-existing Health Insurance Portability and Accountability Act (HIPAA) compliant, clinical image-guided percutaneous ablation prospectively created and maintained database of CLM patients. All CLM patients treated with thermal ablation (RFA and MWA) from 1/2003 to 7/2017 were included in the study. The database was closed for data collection in 7/2018. The main change in ablation procedures during the study period was introduction of immediate post-ablation contrast-enhanced CT (since November 2009). It enabled evaluation of ablation zones and minimal ablation margins, which resulted in significant reduction of local tumor progression rate [45].

\section{Patient selection}

Majority of the patients were considered eligible for ablation following multidisciplinary discussion. However, due to the high workload it was impossible to present all patients in the dedicated multidisciplinary meetings. In these cases the treatment plan was discussed with at least the patient's medical oncologist and most often with surgical oncologist as well.

Inclusion criteria for liver thermal ablation were as follows:

- Age $>18$ years;

- Pathology proven colorectal cancer

- Oligometastatic disease, confined to the liver with up to 3 liver lesions;

- Absent or controllable extrahepatic metastases (up to 5 lesions in 2-3 organ sites);
- Liver recurrence after hepatectomy (majority of the patients were referred for ablation for this reason); OR tumor is unresectable (including cases when patient refused hepatectomy/ resection was challenging due to patient's comorbidities) OR presence of small solitary deep tumor requiring major resection; OR patient was offered local curative therapy by means of ablation instead of surgery within "test of time" concept;

- It is possible to achieve sufficient minimal ablation margins $(\geq 5 \mathrm{~mm})$;

- No abutment of major vascular and biliary structures: tumor is $>1 \mathrm{~cm}$ distance from the major bile ducts (in hilum as well as away from the main right and left biliary ducts);

- Eastern Cooperative Oncology Group performance status of o-2.

- Relative contraindication: no risk of diaphragmatic injury (in subcapsular dome lesions) or surrounding organ damage that could not be protected by hydrodissection.

Patient exclusion criteria are depicted in Figure 1.

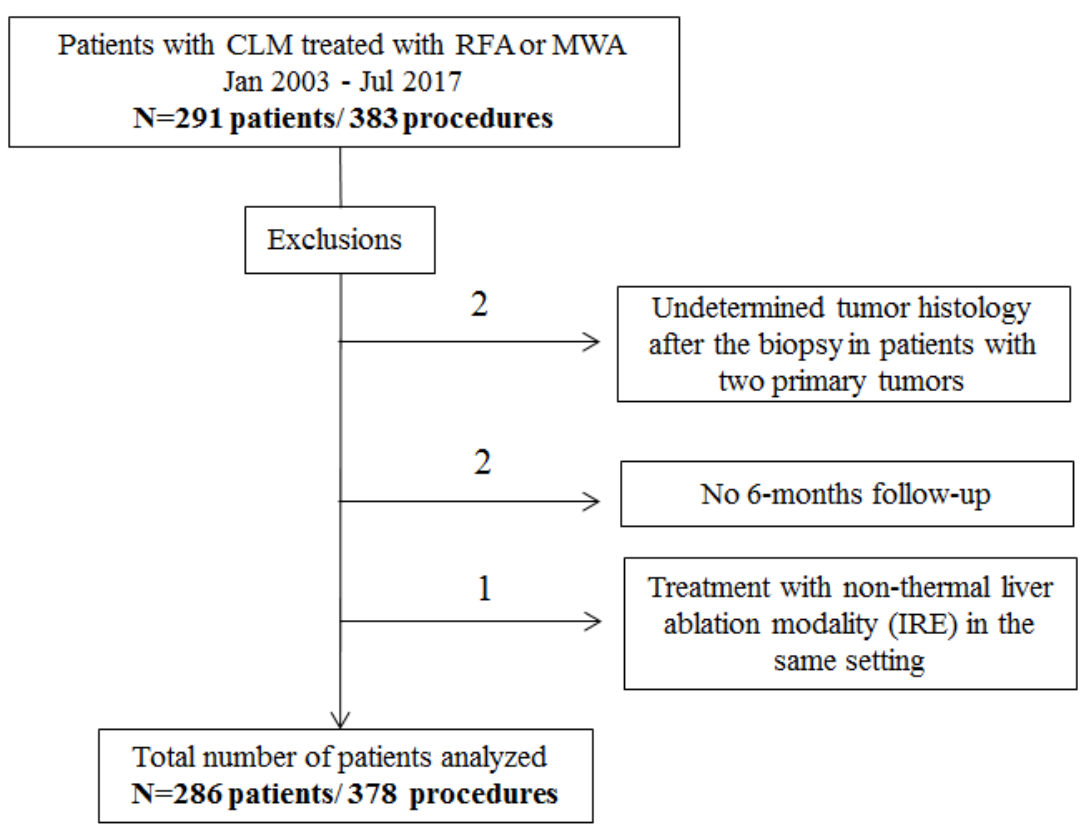

Figure 1 Exclusion criteria. 
Ablation procedure and imaging follow-up

All ablations were performed under general anesthesia, using premedication with cefazolin $1 \mathrm{~g}$ intravenously in a dedicated interventional radiology (IR) CT suite. As of 2009 the dedicated IR suite included CT fluoroscopy and ${ }^{18} \mathrm{~F}-\mathrm{FDG}$ PET/CT capabilities. CT guidance was used for all the procedures. CT fluoroscopy and/or ultrasonography were utilized as needed for real-time needle positioning or ablation zone monitoring. In some of the recent sessions, additional ${ }^{18} \mathrm{~F}$-FDG PET/CT or split-dose technique for ${ }^{18} \mathrm{~F}$-FDG PET/ CT [47] guidance was utilized. Ablation zone monitoring was performed as previously described [45,48]. Since November 2009 all ablations were performed according to manufacturer's protocol with the aim of creating a MM of at least $5 \mathrm{~mm}$ and ideally 10 $\mathrm{mm}$ all around the target tumor.

RF ablations were performed using the following ablation systems: Cool-tip RF electrode (Medtronic, Minneapolis, MN) in 110/213 (51\%) of procedures; RITA Medical Systems (Angiodynamics, Latham, NY) in 52/213 (24\%) and LeVeen Needle electrode (Boston Scientific, Natick, MA) in 39/213 (18\%); for 12/213 (6\%) of procedures data was not available.

MW ablations were performed using the following antennae: Neuwave (Ethicon, Johnson EJohnson) in 98/165 (61\%); Emprint (Medtronic, Minneapolis, MN) in 39/165(24\%); Microsulis (Angiodynamics, Latham, NY) in 10/165 (6\%); Amica (Endocare, Health Tronics) in 9/165 (6\%); Medwaves (Medwaves, San Diego, CA) in 1/165 (o.6\%); Perseon (Perseon Corp, Salt Lake City, UT) in 1/165 (o.6\%) and Sota Medical (Cedar Grove, NJ) in 1/165 (o.6\%); for 6/165 (1.8\%) of procedures data was not available.

Contrast-enhanced CT scan was performed at 4-8 weeks post-ablation and served as the new baseline for future comparisons [49]. Subsequent imaging follow-up was performed every 2-4 months with contrast-enhanced CT, whole body ${ }^{18} \mathrm{~F}-\mathrm{FDG}$ PET/CT and/or liver MRI. MWA was introduced into clinical practice in July 2012.

\section{Measurement of MM}

We utilized a manual minimal ablation margin assessment method using anatomical landmarks on pre-and post-ablation CT. This method was chosen as it is more reproducible and found to be associated with local tumor progression in prior studies [46]. Minimal margin assessment was performed by two radiology readers: A. Bendet (faculty body imaging, 7 years of experience) and W. Shady (research fellow, supervised in margin assessment by C.T. Sofocleous IR faculty with 20 years of experience in thermal ablation).

For margin assessment based on anatomical landmarks, pre- and post-ablation portal venous phase $\mathrm{CT}$ images were reviewed side by side to compare the index tumor and the ablation zone/defect [46].

Study objectives

Study objectives were as follows:

1. To analyze local tumor progression-free survival (LTPFS);

2. To measure the rates of side effects, minor and major complications [50,51];

3. To analyze the factors associated with shorter LTPFS and complications. These included HAI, pre-existing biliary dilatation (within 6 weeks of TA), bevacizumab administration within 1 month of TA, prior hepatectomy, MM size (stratified into the following categories: o mm, 1-5 mm, 6-10 $\mathrm{mm}$ and $>10 \mathrm{~mm}$ ), ablation modality (RFA vs. MWA) as well as number and size of ablated tumors.

4. To analyze overall survival and 30-day mortality post-ablation.

\section{Definitions}

Complications were reported procedure-based according to the modified SIR guidelines [50] with side effects reported separately as per TA terminology standardization guidelines [51]. Complications were additionally classified by the time post-procedure and by organ/system. Definitions of side effects, minor and major complications as well as additional classification of complications are described in Supplement 1.

Definitions of residual tumor, LTP as well as analyzed risk factors, such as MM, preexisting biliary dilatation and HAI are also stated in Supplement 1. LTP was reported when there was at least one post-ablation negative imaging study; otherwise it was considered a residual tumor.

\section{Statistical analysis}

Median study follow-up was estimated for survivors. Median overall survival (from the first ablation date) and local tumor progression-free survival were estimated using Kaplan Meier method. Univariate and multivariate logistic regression analyses, adjusted for patient clustering were used to analyze association of risk factors with development of complications. Univariate exact logistic regression analysis was used to analyze predictors with $100 \%$ sensitivity for development of complications. Fisher exact test was used to analyze difference in categorical variables between the two groups. Mann-Whitney test was used to analyze difference between medians of two groups of non-normally distributed variables (FUDR infusion number). Cox proportional hazards model was used to assess difference in overall survival between the patients with major complications and those without complications. Univariate and multivariate competing 
risk analyses adjusted for clustering were used for analysis of factors associated with decreased LTPFS risk to account for the patients who underwent ablation for more than one tumor (death was considered a competing event in this analysis). Statistical analysis was performed using STATA 11 software. P <0.05 was considered significant. Statistical analysis was reviewed by experienced statistician M. Gonen.

\section{RESULTS}

Study population

Two hundred eighty six patients treated from 1/2003 to 7/2017 were included in the study. 169/286 (59\%) of patients were men (mean age 59.4 \pm 12.7 years) and 117/286 (41\%) - women (mean age $59.8 \pm 12$ years) with a mean age for the entire cohort of $59.5 \pm 12.5$ years. Median study follow-up period for survivors was 31 months.

Four hundred fifteen tumors were treated in 378 procedures. 213/378 (56\%) of procedures were performed using RFA and $165 / 378$ (44\%) - MWA. $30 / 378$ (8\%) of procedures were performed in patients with history of prior HAI, 100/378 (26\%) of procedures were performed to treat recurrence after hepatectomy, 175/378 (46\%) - for recurrence after hepatectomy and HAI. The remaining $73 / 378$ (19\%) of patients had no HAI and no prior liver resection history. KRAS mutation status was positive for $117 / 288$ (41\%) of procedures where genetic information was available.

LTPFS and factors associated with it

Median LTPFS was 30.1 months (IQR, 8.1-NR). One-, two- and three-year LTPFS were 67\% (95\% CI, 62\%-71\%), $55 \%$ (95\% CI, 49\%-60\%) and 47\% (95\% CI, 41-53\%).

Increased MM size was statistically significantly associated with LTPFS $((\mathrm{p}<0.001$, $\mathrm{SHR}=0.38$ ), Figure 2). For the entire study population and during the median duration of the 31 months follow-up, a MM over $10 \mathrm{~mm}$ offered optimal tumor control with no LTP, compared to LTP rates of $26 \%$ for MM of 6-10 mm ( $p=0.0001$ ), $60 \%$ for MM 1-5 mm $(\mathrm{p}=0.0001)$ and $79 \%$ for no MM or o $\mathrm{mm}(\mathrm{p}=0.0001)$ (Table 4$)$. LTPFS, stratified by MM size for the HAI and HAI-naïve groups is depicted in Supplement 4

On univariate analysis, additional factors associated with increased local tumor control were: smaller tumor size ( $\mathrm{p}<0.001$, sub-hazard ratio, $\mathrm{SHR}=1.39$ ), history of liver resection for $\mathrm{CLM}(\mathrm{p}=0.017$, SHR=0.67) and prior HAI therapy $(\mathrm{p}=0.032$, SHR=0.7) $($ Table 3$)$.

On multivariate analysis, only three factors remained statistically significant: tumor size $(\mathrm{p}=0.001, \mathrm{SHR}=1.3), \mathrm{MM}(<0.001, \mathrm{SHR}=0.37)$ and history of liver resection $(\mathrm{p}=0.039$, $\mathrm{SHR}=0.64)$

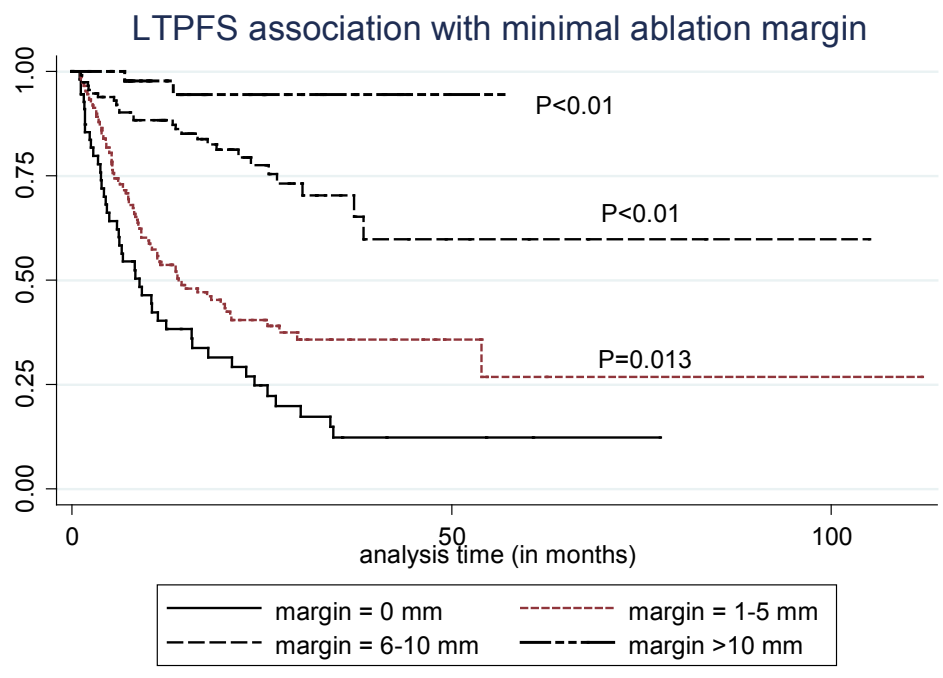

Figure 2 Minimal ablation margin association with local tumor progression-free survival (LTPFS). There were statistically significant differences between LTPFS of patients ablated with o mm minimal margin, compared to $1-5 \mathrm{~mm}$ margin ( $\mathrm{p}=0.013)$, 6-10 $\mathrm{mm}$ margin $(\mathrm{p}<0.01)$ and $>10 \mathrm{~mm}$ minimal margin $(\mathrm{p}<0.01)$

Side effects and complications

Complications were encountered after $70 / 378$ procedures (19\%, 95\% CI 15-23\%). After $49 / 378$ (13\%) of procedures the patients developed minor complications and after $28 / 378$ (7\%) - major complications (7 patients developed both minor and major complications) (Tables 1-2).

Incidence of side effects was 66/378 (10\%) (Supplement 2). Majority of complications (40/70, 57\%) occurred within 24-hours from ablation, 5/40 (13\%) of these were major immediate complications. The incidence of complications in terms of time after TA is depicted in Supplement 3.

Overall complication rates for RFA and MWA were 33/213 (15\%) and 37/165 (19\%), respectively. Major complications occurred after 11/213 (5\%) of RFA and 17/165 (10\%) of MWA procedures $(\mathrm{p}=0.07)$. Among those treated with MWA all major liver complications occurred in patients with prior HAI history $(\mathrm{p}=0.004, \mathrm{OR}=12.01)$. 
Table 1 Post-ablation minor complications

\section{Minor complications post-liver thermal ablatio}

\begin{tabular}{|c|c|}
\hline Complication type/description ( $\mathrm{n}=378$ procedures) & Incidence \\
\hline Biliary & $8(2.1 \%)$ \\
\hline Biloma, no drainage required & 7 \\
\hline $\begin{array}{l}\text { Grade 2-4 hyperbilirubinemia/transaminase toxicity, requiring chemotherapy modification or stop of } \\
\text { HAI therapy }\end{array}$ & 1 \\
\hline Vascular/hemorrhagic complications & $10(2.6 \%)$ \\
\hline Grade 1-2 intrahepatic hematoma, no intervention required & 6 \\
\hline Portal vein injury|thrombosis \pm infarction of corresponding liver, asymptomatic & 2 \\
\hline AV fistula, no intervention required & 1 \\
\hline Perihepatic bleeding during ablation, required $<48 \mathrm{~h}$ observation & 1 \\
\hline $\begin{array}{l}\text { Pulmonary complications } \\
\end{array}$ & $22(5.8 \%)$ \\
\hline Grade 2-3 pneumothorax, requiring thoracostomy & 17 \\
\hline Pleural effusion, requiring thoracentesis & 4 \\
\hline Asthma exacerbation + dyspnea/ desaturation, required hospitalization for $48 \mathrm{~h}$ & 1 \\
\hline Other & $12(3.2 \%)$ \\
\hline Abdominal/non-cardiac chest pain/shoulder pain, required $<48$ hospitalization & 5 \\
\hline Post-ablation syndrome & 3 \\
\hline Nausea/vomiting \pm sedation, requiring $<48 \mathrm{~h}$ observation & 1 \\
\hline Syncope, vomiting, sinus bradycardia, bifascicular blockade next day post-TA, hospitalization for 48 hours & 1 \\
\hline Grade 3 hypertension + hyperglycemia in patient with risk factors,48h admission & 1 \\
\hline Grade 2 fever, negative microbiology cultures & 1 \\
\hline Total number of minor complications & 52 \\
\hline Total number of procedures with minor complication $(s)^{*}$ & $49(13 \%)$ \\
\hline
\end{tabular}

\section{Total number of procedures with minor complication(s)} $49(13 \%)$

incidence of procedures with minor complication(s) is 49 instead of 52 ; HAI-hepatic artery infusion

Among the patients who developed major complication following ablation of a single liver metastasis $(\mathrm{n}=22)$ median extrahepatic progression-free survival was 40.8 months (IQR, 15.7-143).

\section{Biliary complications}

Major complications are detailed in Table 2. Of those, biliary complications were the most commonly encoutered (15/378, 4\%). The most common major biliary complication was intrahepatic biloma requiring drainage $(6 / 378,2 \%)$, with additional subsequent sequelae in 5 out of these 6 cases, including abscess, biliary stricture, bile leak, bacteremia and biliary fistulas to abdominal wall or $\mathrm{m}$. ileopsoas. One of these bilomas developed within an ablation zone previously complicated by hematoma and pseudoaneurysm requiring embolization.

Two more patients developed biliogastric and bilioenteric fistula, requiring intervention, which were not associated with biloma. Total of 3/4 of the patients with biliary fistulas and $1 / 2$ of the patients with arterioportal fistulas requiring intervention received bevacizumab less than a month before (and/or) after the ablation.

Table 2 Post-ablation major complication

$$
\text { Major complica }
$$

post-liver thermal ablation

Intrahepatic biloma, requiring drainage with additional sequelae: abscess (1)/abscess+ secondary biliary stricture + biliary leak (1)/ bacteremia (1)/ biliary fistula to abdominal wall (1) or biliary fistula to $\mathrm{m}$.

ileopsoas (1)

Secondary biliary stenosis, requiring intervention

$\begin{array}{ll}\text { Secondary biliary stenosis, requiring intervention } & 3 \\ \text { Hyperbilirubinemia, requiring stenting }(1) / \text { requiring intervention and prolonged hospitalization }(1) / & 3\end{array}$

resulting in baction

(1)

Extrahepatic biloma + infection + bile leak, requiring intervention

(1) $7(1.9 \%)$

Arterioportal fistula, requiring embolization Liver vascular complications

Hepatic pseudoaneurysm \pm hematoma, requiring embolization

Retroperitoneal hematoma, requiring drainage

Bleeding into biliary tree

Pulmonary complication

Pulmonary embolism \pm pneumonia/bronchiolitis

Pneumothorax \pm pleural effusion, requiring prolonged admission

SVC thrombosis/syndrome + pulmonary vein thrombosis + pulmonary embolism

Hemothorax, requiring thoracostomy and prolonged hospitalization

Pleural effusion + desaturation, requiring thoracentesis + prolonged hospitalization

Diaphragm injury + abscess + hydropneumothorax, requiring $>48 \mathrm{~h}$ hospitalization

(1) hydropneumoperitoneum, requiring $>48$ h hospitalization

\begin{tabular}{cc} 
Other & $\mathbf{2 ( 0 . 5 \% )}$ \\
\hline In alcohol abuse patient SIADH+ Alcohol withdrawal syndrome + epistaxis, requiring intervention and & 1
\end{tabular} prolonged hospitalization

Grade 3 abdominal pain + opioid-induced agitation/delirium with prolonged hospitalization

Total number of major complications

Total number of procedures with major complication(s)*

"After five $\quad \mathbf{2 8}(\mathbf{7 . 4 \% )}$ "After five procedures patients developed two different major complications per procedure, this is why the total diuretic hormone secretion.

Factors associated with biliary complications

Major hepatic complications rates (both biliary and vascular) in HAI patients (208/378) and HAI-naïve patients (170/378) were 18/208 (9\%) vs. 1/17o (o.6\%), respectively ( $\mathrm{p}=0.0002$ ). All biliary complications (both minor and major) occurred only in HAI patients ( $\mathrm{p}<0.001$, $\mathrm{OR}=28.4$ ), thus prior HAI history could predict biliary complications with $100 \%$ sensitivity. 22/208 (11\%) of HAI patients developed some type of biliary complication following TA. Major biliary complications rate in the HAI group was 12/208 (6\%), compared to o/170 
(o\%) for HAI-naïve patients ( $\mathrm{p}=0.0001$ ). HAI and HAI-naïve patients' characteristics are depicted in Supplement 5.

Additional predictors of biliary complications in HAI patients on univariate analysis included pre-existing biliary dilatation $(\mathrm{p}=0.002, \mathrm{OR}=7.2)$, bevacizumab exposure < 1 month before TA ( $\mathrm{p}=0.001, \mathrm{OR}=9.5)$ and larger MM size (assessed as ordinal variable, $\mathrm{p}=0.007, \mathrm{OR}=2.1$ ), all of which retained significance on multivariate analysis (Table 3). In the HAI group, incidence of biliary complications in patients with pre-existing biliary dilatation was $19 / 89$ (21\%, with major biliary complications rate of $17 \%$ ), in patients with recent bevacizumab exposure - $6 / 13$ (46\%, all were major complications) and in the patients ablated with $M M>10 \mathrm{~mm}-9 / 29$ (31\%, with major biliary complications rate of 21\%) (Table 3).

Table 3 Factors associated with biliary and hepatic vascular complications

\begin{tabular}{|c|c|c|c|c|c|c|c|c|c|c|}
\hline \multirow[t]{3}{*}{$\begin{array}{l}\text { Complication risk } \\
\text { factors }\end{array}$} & \multicolumn{4}{|c|}{ Biliary complications } & \multirow{2}{*}{\multicolumn{2}{|c|}{$\begin{array}{c}\begin{array}{c}\text { Vascular } \\
\text { complications }\end{array} \\
\text { UVA }\end{array}$}} & \multicolumn{4}{|c|}{ Local tumor progression } \\
\hline & \multicolumn{2}{|c|}{ UVA } & \multicolumn{2}{|c|}{ MVA } & & & \multicolumn{2}{|c|}{ UVA } & \multicolumn{2}{|c|}{ MVA } \\
\hline & $p$ & $\mathrm{OR}$ & $p$ & OR & \multicolumn{2}{|c|}{$\mathrm{OR}$} & $p$ & SHR & $p$ & SHR \\
\hline Prior HAI therapy & 0.003 & $20.0^{* *}$ & & & 0.2 & 2.0 & 0.032 & 0.7 & 0.86 & 0.96 \\
\hline $\begin{array}{l}\text { Pre-existing biliary } \\
\text { dilatation** }\end{array}$ & 0.002 & 7.2 & 0.005 & 5.8 & - & - & - & - & 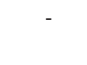 & - \\
\hline $\begin{array}{l}\text { Bevacizumab within } 1 \\
\text { month* }\end{array}$ & 0.001 & 9.5 & 0.017 & 14 & 0.07 & 4.6 & - & - & - & - \\
\hline $\begin{array}{l}\text { Minimal ablation } \\
\text { margins* }\end{array}$ & 0.007 & 2.1 & 0.015 & 2.1 & 0.24 & 1.5 & $<0.001$ & 0.38 & $<0.001$ & 0.37 \\
\hline Ablation modality" & 0.18 & 1.89 & - & - & 0.77 & 1.16 & 0.18 & 0.79 & - & - \\
\hline Tumor size $(\mathrm{cm})^{*}$ & 0.33 & 1.25 & - & - & 0.57 & 0.82 & $<0.001$ & 1.39 & 0.001 & 1.3 \\
\hline Tumor number * & 0.63 & 1.19 & - & - & 0.24 & 1.62 & - & - & - & - \\
\hline $\begin{array}{l}\text { History of liver } \\
\text { resection* }\end{array}$ & 0.14 & 0.40 & - & - & 0.86 & 0.90 & 0.017 & 0.67 & 0.039 & 0.64 \\
\hline $\begin{array}{l}\text { Number of FUDR } \\
\text { infusions*(range, 1-40) }\end{array}$ & 0.37 & 1.03 & - & - & - & & - & - & - & - \\
\hline KRAS mutation status & & - & & - & - & - & 0.49 & 1.15 & - & - \\
\hline
\end{tabular}

'These factors association with biliary complications analysis was made only for the HAI patients' subgroup, since biliary complications occurred only in HAI patient population. ${ }^{* *}$ Result is based on exact logistic regression analysis.
In bold-statistically significant values. UVA-univariate analysis, MVA-multivariate analysis, HAI-hepatic artery infusion pump therapy; FUDR - fluoxuridine, OR-odds ratio; SHR-sub-hazard ratio.

The median number of FUDR infusions, ablation modality, tumor number, size and prior liver resection did not reach statistical significance regarding rates of biliary complications (Table 3).
Optimal MM size in terms of local tumor control and safety according to the HAI history

Since all biliary complications were recorded only in the HAI patients, additional subgroup analyses of HAI and HAI-naïve patients were performed. In the HAI population biliary complications were more prevalent for patients who had ablations with larger $\mathrm{MM}(\mathrm{p}=0.007, \mathrm{OR}=2.1)$. Specifically, when ablating with $\mathrm{MM}$ of $6-10 \mathrm{~mm}$ vs. $>10 \mathrm{~mm}$ the incidence of minor biliary complications increased from $0 \%$ to $10 \%(\mathrm{p}=0.04)$, incidence of major complications increased from $4 \%$ to $21 \%(\mathrm{p}=0.02)$ whereas the corresponding LTP rates decreased from $24 \%$ to $\mathrm{o} \%(\mathrm{p}=0.0033)$ (Table 4$)$.

There were no biliary complications in HAI-naïve patients, regardless of MM size or any other factor. MM larger than $10 \mathrm{~mm}$ provided optimal local tumor control without additional morbidity in this population: LTP rate was $0 \%$ for MM $>10 \mathrm{~mm}$ compared to $27 \%$ for MM of 6-10 mm ( $p=0.1)$ (Table 4$)$.

Table 4 Biliary complications and local tumor progression rate, stratified based on minimal ablation margin size and HAI history

\begin{tabular}{|c|c|c|c|c|}
\hline Minimal ablation margin & o mm & $1-5 \mathrm{~mm}$ & 6-10 mm & $>10 \mathrm{~mm}$ \\
\hline \multicolumn{5}{|c|}{ Whole patients cohort ${ }^{*}$} \\
\hline Minor biliary complications & $\mathrm{o} / 50(0 \%)$ & $4 / 128(3 \%)$ & o/96 (०\%) & $3 / 40(8 \%)$ \\
\hline Major biliary complications & $0 / 50(0 \%)$ & $6 / 128(5 \%)$ & $2 / 96(2 \%)$ & $6 / 40(15 \%)$ \\
\hline Total biliary complications incidence & o/50 (०\%) & $8 / 128(6 \%)^{* *}$ & $2 / 96(2 \%)$ & $9 / 40(23 \%$ \\
\hline Local tumor progression rate & $41 / 52(79 \%)$ & $79 / 132(60 \%)$ & $25 / 98(26 \%)$ & $\mathrm{o} / 39(0 \%)$ \\
\hline \multicolumn{5}{|c|}{ Prior-HAI patients subgroup analysis } \\
\hline Minor biliary complications & $0 / 22(0 \%)$ & $4 / 71(6 \%)$ & $\mathrm{o} / 53(0 \%)$ & $3 / 29(10 \%)$ \\
\hline Major biliary complications & $0 / 22(0 \%)$ & $6 / 71(8 \%)$ & $2 / 53(4 \%)$ & $6 / 29(21 \%)$ \\
\hline Total biliary complications incidence & $\mathrm{o} / 22(0 \%)$ & $8 / 71(11 \%)^{* *}$ & $2 / 53(4 \%)$ & $9 / 29(31 \%)$ \\
\hline Local tumor progression rate & $18 / 22(82 \%)$ & $38 / 72(53 \%)$ & $13 / 54(24 \%)$ & $0 / 28(0 \%)$ \\
\hline \multicolumn{5}{|c|}{ HAI-naive patients subgroup analysis } \\
\hline Total biliary complication incidence & $0 / 28(0 \%)$ & $\mathrm{o} / 57(0 \%)$ & $0 / 43(0 \%)$ & $\mathrm{o} / 11(0 \%)$ \\
\hline Local tumor progression rate & $23 / 30(77 \%)$ & $41 / 60(68 \%)$ & $12 / 44(27 \%)$ & $\mathrm{o} / 11(0 \%)$ \\
\hline
\end{tabular}

Factors associated with vascular complications

Vascular complications developed after 17/378 (4\%) of ablations and 7/378 (2\%) were major. Vascular complications incidence in the HAI group was 12/208 (6\%) vs. 5/170 (3\%) in HAInaïve patients $(\mathrm{p}=0.22)$. Vascular complications incidence between patients with recent bevacizumab compared to those with no bevacizumab exposure was 3/19 (16\%) vs. 14/359 $(4 \%)$, respectively $(\mathrm{p}=0.07)$ 
None of the other analyzed factors in this study were prognostic of vascular complications (Table 3).

\section{Overall survival}

There was no 30-day post-procedural death. Median OS for entire cohort was 41.5 months since ablation (IQR, 22.6-71.5,). One-, two-, three- and five-year OS rates calculated from the date of ablation were $91 \%, 72 \%, 53 \%$ and $37 \%$, respectively. Median OS of the patients with no complications (or only side effects) and major complications were 43.2 months (IQR, 22-75.6) and 29.3 months (IQR, 10.2-NR), respectively ( $\mathrm{p}=0.09$ ), with OS of 41.6 months (IQR, 26.8-53.3) for patients with minor complications (Figure 3).

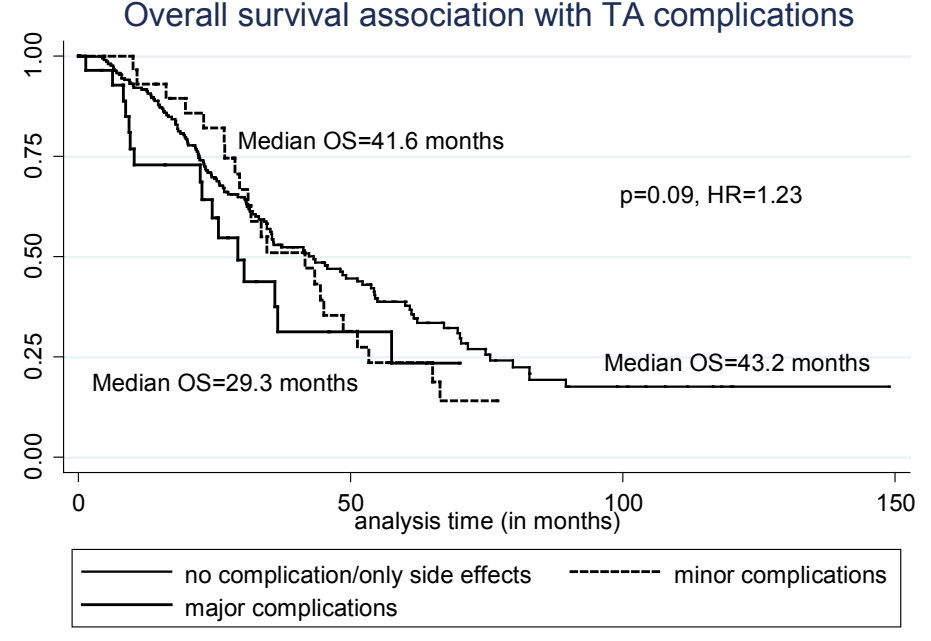

Figure 3 Overall survival association with complications following thermal ablation (TA). The overall survival difference between the patients without complication or with side-effects-only and the patients with major complications did not reach statistical significance $(\mathrm{p}=0.09)$.

\section{DISCUSSION}

To our knowledge, this is the first study to note a strong association between postablation biliary complications with prior HAI as well as with additional risk factors, such as pre-existing biliary dilatation, bevacizumab and MM. Also, this is one of the few studies documenting the importance of MM on long-term tumor control by thermal ablation $[4,45,46,52-56]$ establishing that a $M M>10 \mathrm{~mm}$ was associated with no LTP within a median follow-up of 31 months. On multivariate analysis independent predictors of shorter LTPFS included decreased MM, increased tumor size and no prior history of hepatectomy for CLM similar to prior results [45], accounting for multiple lesions and multiple procedures per patient.

Biliary complications were the most common major complications, encountered after $3 \%$ of all ablations. Prior HAI carried $100 \%$ sensitivity in predicting biliary complications in this study: all patients with biliary complications (both minor and major) had prior HAI exposure.

For HAI-naïve patients optimal oncologic outcomes were safely achieved with MM over $10 \mathrm{~mm}$ (no LTP without biliary complications and minimal morbidity). In the HAI population a MM of $>10 \mathrm{~mm}$ offered optimal local tumor control without any LTP, but it was associated with significantly higher incidence of overall and major biliary complications. Additional predictors for biliary complications in HAI patients included pre-existing biliary dilatation (increased biliary complications risk 7-fold) and recent bevacizumab administration (increased risk 9 -fold), all of which retained statistical significance on multivariate analysis.

In the HAI patient population a MM of 6-10 mm offered local tumor control of $76 \%$ with a $4 \%$ major biliary complication risk. Since re-ablation to treat LTP is usually feasible and adjuvant systemic and/or HAI chemotherapy are also available in this group, we consider ablation with MM of 6-10 mm to be a reasonable option for HAI patients, especially when there is a history of prior bevacizumab exposure, pre-existing biliary dilatation or biloma on CT imaging. Further improvement of local tumor control, particularly when the MM is within the 6-10 mm category, can be achieved with tissue confirmation of complete tumor necrosis through core biopsy of the center and MM of the ablation zone [52]. A MM $>5 \mathrm{~mm}$ with ablation zone biopsy negative for residual tumor after RF ablation of CLM resulted in a 30-month LTPFS of 97\%[52]. Thus, biopsy-proven complete ablation with MM of 6-10 mm can potentially decrease both the LTP and complications rates especially in the HAI patients with pre-existing biliary dilatation and prior bevacizumab exposure where a MM of $>10 \mathrm{~mm}$ may carry increased risk.

Intrahepatic biliary dilatation is a known relative contraindication for ablation [13]. Pre-existing intra- or extrahepatic biliary dilatation regardless of location (adjacent or not to the ablation zone) was considered a biliary complication risk factor. However, pre-existing biliary dilatation did not result in biliary complications in the HAI-naïve patient group. HAI was associated with biliary dilatation that is likely the result of 
multiple (occult) biliary strictures secondary to HAI-related biliary duct ischemia, as previously described [35-37]. Thus, dilatation of even one bile duct most likely reflects a more diffuse liver cholestatic injury in the HAI population. This explains why even minor biliary dilatation was associated with increased incidence of biliary complications in the HAI population in this study and can also explain the difference from HCC patients without exposure to HAI, where mild pre-existing biliary dilatation did not increase the risk of post RFA biliary complications [57].

The addition of bevacizumab to HAI resulted in increased biliary toxicity in three separate prospective studies without any evidence of improved progression-free or overall survival [58]. It is also recommended to withhold bevacizumab for at least 28 days prior to and post-elective surgery [59] to prevent complications or delay healing. Although generally such strict restrictions are not recommended prior to TA, for the patients at risk such as the HAI group a similar precaution should be considered. Administration of bevacizumab within 1 month from TA was borderline significant for liver vascular complications in this study $(\mathrm{p}=0.07)$. This is probably due to the small overall number of vascular complications. None of the other analyzed factors were prognostic of vascular complications. Analysis of different potential predictors, such as distance of the target tumor to a major vessel, anticoagulation therapy, number of electrodes used and number of performed overlapping ablations could also be analyzed as potential vascular complications risk factors in future larger studies with more events of vascular complications.

Major complication rates (5\% for RFA and 10\% for MWA) in this cohort fall within previously reported range for RFA (4-33\%) and for MWA (0-19\%) [13].The patients who developed major complications following ablation of a single liver metastasis demonstrated good extrahepatic disease control. This shows that locoregional therapies play an important role in overal disease control in this patient population.

Biloma, requiring intervention was the most common major complication, noted in $2 \%$ of procedures. This rate was higher, than the previously reported $0.9 \%$ rate after RFA for HCC [6o] and is attributed to the HAI effects as described in detail [33-37].

There was no 30-day post-ablation death in this study. Overall survival of patients with major complications was lower when compared to the patients without any complications. This difference, although not significant, likely reflects the known trend of worse oncologic outcomes in CRC patients undergoing resection for CLM when compared to those without complications [61].
KRAS mutation was not associated with local tumor control in our study. The lack of statistical significance may also be attributed to minor discrepancy in KRAS status between the primary tumor and metastatic sites (concordance rate is 93.7\%) [62]. Also it can be attributed and to the fact, that KRAS mutation is not a binary variable in tumors, and that there is a diversity in mutant alleles and variability in gene copy number, which may also contribute to the heterogeneity of clinical outcomes in cancer patients [63].

Study limitations included its single-center retrospective nature with its inherent limitations. Abutment of major vascular and biliary structures was already an exclusion criterion for ablation in our center; this is why it was not included as a potential risk factor in our study. Ablation technical factors, tumor histopathological characteristics [55,56,64], clinical risk score and chemotherapeutic agents post-ablation were not assessed as potential risk factors of poorer oncological outcomes. These factors could be addressed in the future by larger prospective studies with more events to allow for meaningful conclusions.

\section{CONCLUSION}

Thermal ablation of colorectal liver metastases has a favorable safety profile and provides good local tumor control, especially when a MM larger than $5 \mathrm{~mm}$ is achieved. Optimal and sustained local control was documented for $\mathrm{MM}>10 \mathrm{~mm}$ that was associated with no local tumor progression. A larger MM may increase the risk of biliary complications in specific populations at risk such as the patients with prior HAI and particularly those who also have pre-existing biliary dilatation or recent exposure to bevacizumab. For these patients at risk we recommend to hold bevacizumab for 4 weeks before and after ablation and maintaining ablation margins between 6-10 $\mathrm{mm}$.

\section{CLINICAL PRACTICE POINTS}

- Thermal ablation (TA) is a minimally invasive treatment for selected patients with relatively small colorectal cancer liver metastases.

- The most common TA modalities - radiofrequency and microwave ablation - are safe and effective with similar safety profiles, with major complication rates of 0-33\% and pooled morality rate up to $0.23 \%$ when used to treat CLMs.

- Thermal ablation with minimum ablation margins $(M M)>10 \mathrm{~mm}$ offers the best local tumor control and no LTP. 
- In the patients at risk such as those with hepatic artery infusion therapy (HAI) and in particular those with additional bevacizumab exposure and pre-existing biliary dilatation, a $\mathrm{MM}>10$ may further increase the risk of biliary complications.

- MM of 6-10 mm offered $76 \%$ local tumor control with a $4 \%$ incidence of biliary complications in the HAI patient population.

- In HAI-naïve patients, optimal sustained local tumor control is achieved with MM $>10 \mathrm{~mm}$ without complications.

\section{ACKNOWLEDGEMENTS}

We would like to thank Dr. Waleed Shady for contributing to the minimum ablation margin assessment, which was analyzed as oncologic outcomes prognosticator in this manuscript.

The research funded by the Memorial Sloan Kettering Cancer Centre Support Grant/ Core Grant (P30 CAoo8748).

\section{REFERENCES}

1. Elias, D. et al. Percutaneous radiofrequency thermoablation as an alternative to surgery for treatment of liver tumour recurrence after hepatectomy. The British journal of surgery $\mathbf{8 9}, 752$ 756, doi:10.1046/|j.1365-2168.2002.02081.X (2002).

2. Sofocleous, C. T. et al. CT-guided radiofrequency ablation as a salvage treatment of colorectal cancer hepatic metastases developing after hepatectomy. J Vasc Interv Radiol 22, 755-761, doi:10.1016/j. jvir.2011.01.451 (2011)

3. Livraghi, T. et al. Percutaneous radiofrequency ablation of liver metastases in potential candidates for resection: the "test-of-time approach". Cancer 97. 3027-3035, doi:10.1002/cncr.11426 (2003).

4. Shady, W. et al. Percutaneous Microwave versus Radiofrequency Ablation of Colorectal Liver Metastases: Ablation with Clear Margins (Ao) Provides the Best Local Tumor Control. Journal of vascular and interventional radiology: JVR 29, 268-275. e261, doi:10.1016|j.jvir.2017.08.021 (2018)

5. Ruers, T. et al. Local Treatment of Unresectable Colorectal Liver Metastases: Results of a Randomized Phase II Trial. Journal of the National Cancer Institute 109, doi:10.1093/jnci/djxo15 (2017).

6. Jing, X. et al. Complications of thermal ablation of hepatic tumors: Comparison of radiofrequency and microwave techniques. 31, e15040-e15040, doi:10.120o/jco.2013.31.15_suppl.e15040 (2013).

7. Livraghi, T., Meloni, F., Solbiati, L. \& Zanus, G. Complications of microwave ablation for liver tumors: results of a multicenter study. Cardiovascular and interventional radiology 35,868 874, doi: 10.1007/soo270-011-0241-8 (2012).

8. Solbiati, L. et al. Small liver colorectal metastase treated with percutaneous radiofrequency ablation: local response rate and long-term surviva with up to 10-year follow-up. Radiology 265, 958-968, doi:10.1148/radiol.12111851 (2012).

9. Van Tilborg, A. A. et al. Long-term results of radiofrequency ablation for unresectable colorectal liver metastases: a potentially curative intervention. The British journal of radiology 84, 556565, doi:10.1259/bjr/78268814 (2011).

10. Gillams, A. R. \& Lees, W. R. Five-year survival in 309 patients with colorectal liver metastases treated with radiofrequency ablation. European radiology 19, 1206-1213, doi: 10.1007/soo330-008-1258-5 (2009).

11. Hamada, A. et al. Radiofrequency ablation for colorectal liver metastases: prognostic factors in non-surgical candidates. Japanese journal of radiology 30, 567-574, doi: 10.1007/511604-012-0089-0 (2012)

12. Wong, S. L. et al. American Society of Clinical Oncology 2009 clinical evidence review on radiofrequency ablation of hepatic metastases from colorectal cancer. Journal of clinical oncology: official journal of the American Society of Clinical Oncology 28, 493-508, doi:10.120o/jco.2009.23.4450 (2010)

13. Vogl, T. J et al. Thermal ablation of liver metastases from colorectal cancer: radiofrequency, microwave and laser ablation therapies. Radiol Med 119, 451-461, doi: 10.1007/s11547-014-0415-y (2014).

14. Lahat, E. et al. Complications after percutaneous ablation of liver tumors: a systematic review. Hepatobiliary surgery and nutrition 3, 317-323, doi:10.3978|j.issn.2304-3881.2014.09.07 (2014)

15. Fonseca, A. Z., Santin, S., Gomes, L. G., Waisberg, J. \& Ribeiro, M. A., Ir. Complications of radiofrequency ablation of hepatic tumors: Frequency and risk factors. World journal of hepatology 6, 107-113, doi:10.4254/wjh.v6.ij.107 (2014). 
16. Wood, T. F. et al. Radiofrequency ablation of 231 unresectable hepatic tumors: indications, limitations, and complications. Annals of surgical oncology 7, 593-600 (2000)

17. Rhim, H. et al. Major complications after radiofrequency thermal ablation of hepatic tumors: spectrum of imaging findings. Radiographics 23 123-134; discussion 134-126, doi:10.1148/rg.231025054 (2003)

18. Bowles, B. J. et al. Safety and efficacy of radiofrequency thermal ablation in advanced liver tumors. Archives of surgery (Chicago, Ill.: 1960) 136, 864-869 (2001).

19. Francica, G., Marone, G., Solbiati, L., D’Angelo, V. \& Siani, A. Hemobilia, intrahepatic hematoma and acute thrombosis with cavernomatous transformation of the portal vein after percutaneous thermoablation of a liver metastasis. European radiology 10, 926-929, doi: 10.1007| so03300051038 (2000).

20. Shankar, S., van Sonnenberg, E., Silverman, S. G., Tuncali, K. \& Morrison, P. R. Diagnosis and treatment of intrahepatic biloma complicating radiofrequency ablation of hepatic metastases AJR. American journal of roentgenology 181, 475-477, doi:10.2214/ajr.181.2.1810475 (2003).

21. Ogawa, T. et al. Prevention of biliary complication in radiofrequency ablation for hepatocellula carcinoma-Cooling effect by endoscopic nasobiliary drainage tube. European journal of radiology 73, 385-39o, doi:10.1016/j.ejrad.2008.10.021 (2010).

22. Goto, E. et al. Hemorrhagic complications of percutaneous radiofrequency ablation for live tumors. Journal of clinical gastroenterology 44, 374380, doi:10.1097/MCG.obo13e3181b7ed76 (2010).

23. D'Angelica, M. I. et al. Phase II trial of hepatic artery infusional and systemic chemotherapy for patients with unresectable hepatic metastases from colorectal cancer: conversion to resection and long-term outcomes. Annals of surgery 261, 353-360, doi:10.1097/sla.ooooooooooooo614 (2015).

24. Pak, L. M. et al. Prospective phase II trial of combination hepatic artery infusion and systemic chemotherapy for unresectable colorectal liver metastases: Long term results and curative potential. Journal of surgical oncology 117, 634-643, doi:10.1002/jso.24898 (2018).

25. Kanat, O., Gewirtz, A. \& Kemeny, N. What is the potential role of hepatic arterial infusion chemotherapy in the current armamentorium against colorectal cancer. Journal of gastrointestinal oncology 3, 130-138, doi:10.3978/j.issn.2078-6891.2011.025 (2012).

26. Bupathi, M., Ahn, D. H. \& Bekaii-Saab, T. Spotlight on bevacizumab in metastatic colorectal cancer: patient selection and perspectives. Gastrointestinal cancer: targets and therapy 6, 21-30, doi:10.2147/gictt. s97740 (2016).

27. Benson, A. B., 3rd et al. Colon Cancer, Version 1.2017 NCCN Clinical Practice Guidelines in Oncology. Journal of the National Comprehensive Cancer Network: JNCCN 15, 370-398 (2017)

28. Van Cutsem, E. et al. ESMO consensus guidelines for the management of patients with metastatic colorectal cancer. Annals of oncology: official journal of the European Society for Medical Oncology 27, 13861422, doi:10.1093/annonc/mdw235 (2016).

29. Kemeny, N. et al. Phase I trial of adjuvant hepatic arterial infusion (HAI) with floxuridine (FUDR) and dexamethasone plus systemic oxaliplatin, 5 -fluorouracil and leucovorin in patients with resected liver metastases from colorectal cancer. Ann Oncol 20, 1236-1241, doi:10.1093/annonc/mdn769 (2009)

30. Kemeny, N. E. et al. Randomized phase II trial of adjuvant hepatic arterial infusion and systemic chemotherapy with or without bevacizumab in patients with resected hepatic metastases from colorectal cancer. J Clin Oncol 29, 884-889, doi:10.120o/jco.2010.32.5977 (2011)

31. Kemeny, N. E. et al. Hepatic arterial infusion versus systemic therapy for hepatic metastases from colorectal cancer: a randomized trial of efficacy, quality of life, and molecular markers (CALGB 9481).J Clin Oncol 24, 1395-1403, doi:10.1200/ jc0.2005.03.8166 (2006)

32. Kemeny, N. et al. A randomized trial of intrahepatic infusion of fluorodeoxyuridine with dexamethasone versus fluorodeoxyuridine alone in the treatment of metastatic colorectal cancer. Cancer 69, 327-334 (1992).

33. Ito, K. et al. Biliary sclerosis after hepatic arterial infusion pump chemotherapy for patients with colorectal cancer liver metastasis: incidence, clinical features, and risk factors. Ann Surg Oncol 19 , 1609-1617, doi: 10.1245/s10434-011-2102-8 (2012).

34. Karanicolas, P. J. et al. Hepatic arterial infusion pump chemotherapy in the management of colorectal liver metastases: expert consensus statement. Curr Oncol 21, e129-136, doi:10.3747| co.21.1577 (2014)

35. Brown, K. T. et al. Obstructive jaundice in patients receiving hepatic artery infusional chemotherapy: etiology, treatment implications, and complications after transhepatic biliary drainage. Journal of vascular and interventional radiology: JVIR 8, 229-234 (1997).

36. Pien, E. H. et al. Iatrogenic sclerosing cholangitis following hepatic arterial chemotherapy infusion. Radiology 156, 329-330, doi:10.1148| radiology.156.2.3160o62 (1985).

37. Botet, J. F., Watson, R. C., Kemeny, N., Daly, J. M. \& Yeh, S. Cholangitis complicating intraarterial chemotherapy in liver metastasis. Radiology $\mathbf{1 5 6}$,
335-337, doi:10.1148/radiology.156.2.3160o63 (1985).

38. Hillerdal, G. Indolent lung cancers-time for a paradigm shift: a review. Journal of thoracic oncology: official publication of the International Association for the Study of Lung Cancer 3, 208-211, doi:10.1097| JTO.obo13e3181653се3 (2008).

39. Yoshioka, Y. et al. Postoperative complications following neoadjuvant bevacizumab treatment for advanced colorectal cancer. Surg Today 44, 1300-1306, doi: 10.1007/s00595-013--0686-2 (2014).

40. Curley, S. A. et al. Early and late complications after radiofrequency ablation of malignant liver tumors in 608 patients. Annals of surgery 239, 450-458 (2004).

41. Poon, R. T. et al. Learning curve for radiofrequency ablation of liver tumors: prospective analysis of initial 100 patients in a tertiary institution. Annals of surgery 239, 441-449, doi:10.1097/01. sla.o0oo118565.21298.0a (2004).

42. Mulier, S. et al. Complications of radiofrequency coagulation of liver tumours. The British journal of surgery 89, 1206-1222, doi:10.1046/j.13652168.2002.02168.x (2002).

43. Kong, W. T. et al. Major complications after radiofrequency ablation for liver tumors: analysis of 255 patients. World journal of gastroenterology 15 , 2651-2656 (2009).

44. Howenstein, M. J. \& Sato, K. T. Complications of radiofrequency ablation of hepatic, pulmonary, and renal neoplasms. Seminars in interventional radiology 27, 285-295, doi: 10.1055/s-0030-1261787 (2010).

45. Shady, W. et al. Percutaneous Radiofrequency Ablation of Colorectal Cancer Liver Metastases: Factors Affecting Outcomes-A 10-year Experience at a Single Center. Radiology 278, 601-611, doi:10.1148 radiol.2015142489 (2016)

46. Wang, X. et al. Margin size is an independent predictor of local tumor progression after ablation 
of colon cancer liver metastases. Cardiovascular and interventional radiology 36, 166-175, doi: 10.1007| soo270-012-0377-1 (2013).

47. Ryan, E. R. et al. Split-dose technique for FDG PET/ CT-guided percutaneous ablation: a method to facilitate lesion targeting and to provide immediate assessment of treatment effectiveness. Radiology 268, 288-295, doi:10.1148/radiol.13121462 (2013).

48. Cornelis, F. et al. 18F-FDG PET/CT Is an Immediate Imaging Biomarker of Treatment Success After Liver Metastasis Ablation. Journal of nuclear medicine: official publication, Society of Nuclear Medicine 57, 1052-1057, doi:10.2967/jnumed.115.171926 (2016)

49. Pua, B. B. \& Sofocleous, C. T. Imaging to optimize liver tumor ablation. Imaging in medicine 2, 433-443. doi:10.2217/iim.10.32 (2010)

50. Sacks, D., McClenny, T. E., Cardella, J. F. \& Lewis, C. A. Society of Interventional Radiology clinical practice guidelines. Journal of vascular and interventional radiology: JVIR 14, S199-202 (2003).

51. Ahmed, M. et al. Image-guided tumor ablation standardization of terminology and reporting criteria-a 10-year update. Radiology 273, 241-260, doi:10.1148/radiol.14132958 (2014)

52. Sotirchos, V. S. et al. Colorectal Cancer Liver Metastases: Biopsy of the Ablation Zone and Margins Can Be Used to Predict Oncologic Outcome. Radiology 280, 949-959, doi:10.1148/ radiol.2016151005 (2016).

53. Kaye, E. A. et al. Volumetric $3 \mathrm{D}$ assessment of ablation zones after thermal ablation of colorectal liver metastases to improve prediction of local tumor progression. European radiology, doi: 10.1007 so0330-018-5809-0 (2018).

54. Cornelis, F. H. et al. Immediate Postablation (18) F-FDG Injection and Corresponding SUV Are Surrogate Biomarkers of Local Tumor Progression
After Thermal Ablation of Colorectal Carcinoma Liver Metastases. Journal of nuclear medicine: official publication, Society of Nuclear Medicine 59, 1360-1365, doi:10.2967/jnumed.117.194506 (2018).

55. Odisio, B. C. et al. Local tumour progression after percutaneous ablation of colorectal liver metastases according to RAS mutation status. The British journal of surgery 104, 760-768, doi:10.1002/ bjs.1049o (2017).

56. Calandri, M. et al. Ablation of colorectal liver metastasis: Interaction of ablation margins and RAS mutation profiling on local tumour progression-free survival. European radiology $\mathbf{2 8}$, 2727-2734, doi: 10.1007/soo330-017-5273-2 (2018).

57. Kondo, Y. et al. Intrahepatic bile duct dilatation after percutaneous radiofrequency ablation for hepatocellular carcinoma: impact on patient's prognosis. Liver international: official journal of the International Association for the Study of the Liver 31, 197-205, doi:10.1111/j.1478-3231.2010.02415.x (2011).

58. Cercek, A. et al. Floxuridine hepatic arterial infusion associated biliary toxicity is increased by concurrent administration of systemic bevacizumab. Annals of surgical oncology 21, 479-486, doi: 10.1245/1510434-013-3275-o (2014)

59. Genetech, I. U.S. BL 125085/169 Amendment: Bevacizumab-Genentech. San Francisco, CA, USA (2009).

6o. Chang, I. S. et al. Biloma formation after radiofrequency ablation of hepatocellular carcinoma: incidence, imaging features, and clinical significance. AJR Am J Roentgenol 195, 11311136, doi:10.2214/ajr.09.3946 (2010)

61. Correa-Gallego, C. et al. Perioperative complications influence recurrence and survival after resection of hepatic colorectal metastases. Annals of surgical oncology 20, 2477-2484, doi: 10.1245/s10434-013-2975-9 (2013)
62. Bhullar, D. S. et al. Biomarker concordance between primary colorectal cancer and its metastases. EBioMedicine 40, 363-374, doi:10.1016/j. ebiom.2019.01.050 (2019).

63. Janakiraman, M. et al. Genomic and biological characterization of exon 4 KRAS mutations in human cancer. Cancer research 70, 5901-5911, doi:10.1158/ooo8-5472.Can-10-0192 (2010).

64. Shady, W. et al. Kras mutation is a marker of worse oncologic outcomes after percutaneous radiofrequency ablation of colorectal liver metastases. Oncotarget 8, 66117-66127, doi:10.18632| oncotarget.19806 (2017). 


\section{SUPPLEMENTARY MATERIAL}

Supplement 1 Definitions of parameters of local tumor control and adverse event

\begin{tabular}{|c|c|}
\hline Parameter & Description \\
\hline \multicolumn{2}{|r|}{ Local tumor control } \\
\hline Residual tumor & $\begin{array}{l}\text { Residual tumor and ablation failure was considered to be the imaging evidence of tumor (FDG- } \\
\text { avid or nodular enhancement) on the first imaging at } 4-8 \text { weeks post-ablation }\end{array}$ \\
\hline $\begin{array}{l}\text { Local tumor } \\
\text { progression (LTP) }\end{array}$ & $\begin{array}{l}\text { Defined as any new FDG-uptake ( } \mathrm{SUV}_{\text {max }} 2.5 \text { above background liver uptake) or peripheral// } \\
\text { nodular enhancement within } 1 \text {-cm of the ablation zone or enlargement of the baseline ablation } \\
\text { defect, depicted during imaging follow-up on any subsequent CT and/or ris-FDG PET/CT imaging } \\
\text { after ablation. LTP was reported when there was at least one post-ablation negative imaging } \\
\text { study; otherwise it was considered a residual tumor }\end{array}$ \\
\hline \multicolumn{2}{|r|}{ Adverse events } \\
\hline Side effects & $\begin{array}{l}\text { Side effects included expected, undesired consequences of the procedure that occur commonly, } \\
\text { but do not lead to an unexpected increased level of care. These included pain, post-ablation } \\
\text { syndrome and asymptomatic imaging evidence of pleural effusion/perihepatic fluid or blood } \\
\text { as well as imaging findings of thermal damage to adjacent structures without sequelae, nor } \\
\text { requiring hospitalization and absent on first post-TA imaging }\end{array}$ \\
\hline Minor complications & $\begin{array}{l}\text { Minor complications included exacerbation of comorbidities requiring }<48 \text { hours } \\
\text { hospitalization, imaging evidence of biloma, hematoma or arteriovenous fistula on imaging } \\
\text { follow-up, not requiring intervention or hospitalization and not resulting in significant } \\
\text { morbidity. They also included laboratory toxicity, requiring modification of systemic } \\
\text { chemotherapy or HAl therapy administration }\end{array}$ \\
\hline Major complications & $\begin{array}{l}\text { Major complications included events that led to substantial morbidity and disability, resulting } \\
\text { in }>48 \text { hours hospital admission, substantially lengthening the hospital stay, requiring blood } \\
\text { transfusion, intervention or interruption of systemic chemotherapy administration }\end{array}$ \\
\hline $\begin{array}{l}\text { Complications } \\
\text { timing post- } \\
\text { procedure }\end{array}$ & $\begin{array}{l}\text { Complications were classified as follows: } \\
\text { Immediate complications ( } 6 \text {-24h post-ablation) } \\
\text { Early complications ( } 24 \text { h }-30 \text { days post-ablation) } \\
\text { Delayed complications }(>30 \text { days }-6 \text { months post-ablation })\end{array}$ \\
\hline $\begin{array}{l}\text { Complications } \\
\text { by organ }\end{array}$ & $\begin{array}{l}\text { Complications were classified as follows: hepatic (biliary and vascular); pulmonary and other } \\
\text { complications }\end{array}$ \\
\hline $\begin{array}{l}\text { Classification of } \\
\text { pneumothorax and } \\
\text { pleural effusion }\end{array}$ & $\begin{array}{l}\text { Pneumothorax and pleural effusion were classified according to the level of severity. They were } \\
\text { considered: } \\
\text { Side effects if they did not require thoracostomy/thoracentesis } \\
\text { Minor complication if they required thoracostomy/thoracentesis } \\
\text { Major complication if they required thoracostomy/thoracentesis with }>48 \text { hours of } \\
\text { hospitalization and/or had further sequelae }\end{array}$ \\
\hline \multicolumn{2}{|r|}{ Risk factors } \\
\hline $\begin{array}{l}\text { Minimum ablation } \\
\text { margin (MM) }\end{array}$ & $\begin{array}{l}\text { The MM was measured on the first contrast-enhanced triphasic CT scan at } 4-8 \text { weeks post-ablation } \\
\text { and compared to the pre-ablation contrast-enhanced triphasic CT scan obtained prior to TA as } \\
\text { previously described } 44,454,46 \text {. MM was stratified into the following categories: } 0 \text { mm, } 1-5 \mathrm{~mm} \text {, } \\
6-10 \mathrm{~mm} \text { and }>10 \mathrm{~mm} \text {. The margins were measured at } 4-8 \text { weeks post-TA instead of immediate } \\
\text { post-treatment CT in order to keep evaluations uniform due to immediate post-procedure } \\
\text { contrast-enhanced CT introduction in our institution only in November } 2009 \text {. } \\
\text { The MM was assessed by two radiology readers: A. Bendet (faculty body imaging, } 7 \text { years of } \\
\text { experience) and W. Shady (research fellow, supervised in margin assessment by C.T. Sofocleous- } \\
\text { IR faculty with } 20 \text { years of experience in thermal ablation) }\end{array}$ \\
\hline $\begin{array}{l}\text { Pre-existing biliary } \\
\text { dilatation }\end{array}$ & $\begin{array}{l}\text { Pre-existing biliary dilatation was defined as dilatation of at least one intra- or extra-hepatic } \\
\text { biliary duct, depicted on contrast-enhanced CT or MRI within } 6 \text { weeks before the ablation. It } \\
\text { was assessed by faculty radiologist with at least 7-years of experience in diagnostic radiology }\end{array}$ \\
\hline $\begin{array}{l}\text { Hepatic artery } \\
\text { infusion (HAI) }\end{array}$ & $\begin{array}{l}\text { HAI therapy with fluoxuridine (FUDR) was analyzed in this study, with dexamethasone being } \\
\text { administered at the same setting with FUDR to reduce liver toxicity }\end{array}$ \\
\hline
\end{tabular}

Supplement 2 Post-ablation side effects

\section{Side effects $(\mathrm{n}=378)$}

Incidence

Grade 1-2 abdominal/chest/shoulder pain, no observation required

Prethothona, required $<48$ h observation (iatrogenic $=2$ )

Trace subcapsular/perihepatic fluid/blood collection,

$16(4 \%)$
$7(2 \%)$

no observation/intervention required and not present on follow-up

Trace pleural effusion with mild chest pain/dyspnea, not affecting daily function/no observation/ $\quad 6(2 \%)$

treatment required and not present on follow-up

Grade 1-2 nausea/vomiting

Grade 2 constipation

Hematoma/skin swelling/induration in ablation zone

Mild fever

Hypoxia, requiring overnight observation

Throat soreness/irritation

Minimal bleeding in ablation zone, asymptomatic, not present on follow-up

Subcutaneous emphysema, required $<48$ h observation

Portal hypertension

Hiccups (RFA in proximity to mediastinum)

Dyspepsia

Grade 2 hyperbilirubinemia

Eyelids edema/ abrasion/swelling

\section{Total number of side effects encountered}

Total number of procedures with side effects reported ${ }^{*}$ is lower than the number of side effects encoutered per se

Supplement 3 Post-thermal ablation complications distribution by time post-procedure Complications ( $\mathbf{n}=378$, according to SIR guidelines, 2003)

Immediate complications (6-24h post-ablation) of Incidence

Early complications (within 30 days post-ablation)

Delayed complications ( $>30$ days -6 months post-ablation)

Complications $>6$ months post-ablation 
Supplement 4 Local tumor progression-free survival (LTPFS) association with minimal ablation margin (MM) and history of hepatic artery chemotherapy (HAI). CI-confidence interval.

\begin{tabular}{llll}
\hline Minimal margin category & Entire cohort, 95\%CI & HAI-naïve, 95\%CI & HAI patients, 95\%CI \\
\hline MM = omm & & & \\
1-year LTPFS & $38 \%(25 \%-51 \%)$ & $32 \%(16 \%-49 \%)$ & $45 \%(24 \%-64 \%)$ \\
2-year LTPFS & $23 \%(12 \%-35 \%)$ & $25 \%(11 \%-42 \%)$ & $18 \%(5 \%-37 \%)$ \\
3 -year LTPFS & $10 \%(3 \%-21 \%)$ & $8 \%(2 \%-23 \%)$ & $12 \%(2 \%-31 \%)$ \\
MM = 1-5mm & & & \\
1-year LTPFS & $53 \%(45 \%-61 \%)$ & $53 \%(40 \%-64 \%)$ & $53 \%(42 \%-64 \%)$ \\
2-year LTPFS & $40 \%(31 \%-48 \%)$ & $31 \%(19 \%-44 \%)$ & $47 \%(35 \%-58 \%)$ \\
3-year LTPFS & $35 \%(27 \%-44 \%)$ & $28 \%(16 \%-41 \%)$ & $42 \%(29 \%-53 \%)$ \\
MM = 6-10mm & $88 \%(80 \%-93 \%)$ & $85 \%(71 \%-93 \%)$ & $91 \%(80 \%-96 \%)$ \\
1-year LTPFS & $76 \%(65 \%-84 \%)$ & $78 \%(61 \%-88 \%)$ & $75 \%(59 \%-85 \%)$ \\
2-year LTPFS & $69 \%(56 \%-79 \%)$ & $73 \%(54 \%-85 \%)$ & $65 \%(45 \%-79 \%)$ \\
3-year LTPFS & & & \\
MM >10 mm & & $100 \%(-)$ & $100 \%(-)$ \\
1-year LTPFS & $100 \%(-)$ & $100 \%(-)$ & $100 \%(-)$ \\
2-year LTPFS & $100 \%(-)$ & $100 \%(-)$ & $100 \%(-)$ \\
3-year LTPFS & $100 \%(-)$ & & \\
\hline
\end{tabular}

Supplement $\mathbf{5}$ Comparison on the patients with prior hepatic artery infusion therapy (HAI) and HAInaïve patients

\begin{tabular}{|c|c|c|c|}
\hline Parameter & HAI patients & HAI-naïve patients & P-value \\
\hline Number of patients & 140 & 146 & - \\
\hline Number of procedures & 208 & 170 & - \\
\hline Age & $57.1 \pm 11$ years & $62.9 \pm 13.4$ & - \\
\hline Gender & $88 / 140(63 \%)$ male & $82 / 147(56 \%)$ male & - \\
\hline Number of FUDR infusions, median & 8 (range, $1-40)$ & - & - \\
\hline Pre-existing biliary dilatation & $89 / 196(45 \%)$ & $26 / 156(17 \%)$ & 0.0001 \\
\hline \multicolumn{4}{|c|}{ Incidence of biliary complications } \\
\hline Minor & $8 / 208(4 \%)$ & $0 / 170(0 \%)$ & 0.0094 \\
\hline Major & $15 / 208(7 \%)$ & $0 / 170(0 \%)$ & 0.0002 \\
\hline Total & $22 / 208^{*}(11 \%)$ & $0 / 170(0 \%)$ & 0.0001 \\
\hline \multicolumn{4}{|c|}{ Incidence of vascular complications: } \\
\hline Minor & $6 / 208(3 \%)$ & $4 / 170(2 \%)$ & 1 \\
\hline Major & $6 / 208(3 \%)$ & $1 / 170(0.6 \%)$ & 0.63 \\
\hline Total & $12 / 208(6 \%)$ & $5 / 170(3 \%)$ & 0.59 \\
\hline \multicolumn{4}{|c|}{ Number of patients who received bevacizumab within 1-months of TA } \\
\hline Total bevacizumab administrations within 1-month of TA & $11 / 140(8 \%)$ & $6 / 147(4 \%)$ & 0.21 \\
\hline Bevacizumab administered simultaneously with HAI & 4/140 (3\%) & - & - \\
\hline Bevacizumab administered not simultaneously with HAI & $5 / 140(4 \%)$ & - & - \\
\hline Data is on bevacizumab and HAI timing not available & $2 / 140(1 \%)$ & $0 / 147(0 \%)$ & - \\
\hline \multicolumn{4}{|c|}{ Minimum ablation margin sizes } \\
\hline $\mathrm{o} \mathrm{mm}$ & $22 / 208(11 \%)$ & $28 / 170(16 \%)$ & 0.096 \\
\hline $1-5 \mathrm{~mm}$ & $71 / 208(34 \%)$ & $57 / 170(34 \%)$ & 0.91 \\
\hline 6-10 mm & $53 / 208(25 \%)$ & $43 / 170(25 \%)$ & 1 \\
\hline$>10 \mathrm{~mm}$ & $29 / 208(14 \%)$ & $11 / 170(6 \%)$ & 0.02 \\
\hline Not applicable* $^{* *}$ & $33 / 208(16 \%)$ & $31 / 170(18 \%)$ & - \\
\hline \multicolumn{4}{|c|}{ Local tumor progression-free survival (LTPFS) } \\
\hline Median (in months, from TA date) & Not reached & 21 months & 0.026 \\
\hline 1-year LTPFS & $71 \%(95 \% \mathrm{Cl}, 64 ; 77 \%)$ & $62 \%(95 \% \mathrm{Cl}, 54 ; 69 \%)$ & - \\
\hline 2-year LTPFS & $60 \%(95 \% \mathrm{CI}, 52 ; 66 \%)$ & $49 \%(95 \% \mathrm{Cl}, 40 ; 57 \%)$ & \\
\hline 3-year LTPFS & $53 \%(95 \% \mathrm{Cl}, 44 ; 60 \%)$ & $41 \%(95 \% \mathrm{CI}, 32 ; 50 \%)$ & - \\
\hline
\end{tabular}


Factors Affecting Oncologic Outcomes of ${ }^{\circ \circ} \mathrm{Y}$ Radioembolization of Heavily Pre-Treated Patients with Colon Cancer Liver Metastases

Kurilova I., Beets-Tan R. G. H., Flynn J., Gonen M., Ulaner G., Petre N. E., Boas F. E., Ziv E., Yarmohammadi H., Klompenhouwer E.G., Cercek A., Kemeny N.A., Sofocleous C.T. 


\section{ABSTRACT}

Objectives

To identify predictors of overall (OS) and liver progression-free survival (LPFS) following ${ }^{90} \mathrm{Y}$ radioembolization (RE) of heavily pretreated patients with colorectal cancer liver metastases (CLM). Create and validate a predictive nomogram for OS.

\section{Materials and Methods}

Metabolic, anatomic, laboratory, pathologic, genetic, primary-disease and procedurerelated factors, as well as pre- and post-RE therapies in 103 CLM patients treated with RE from 9/15/2009 to 3/21/2017 were analyzed. LPFS was defined by RECIST1.1 and EORTC criteria. Prognosticators of OS and LPFS were selected using univariate Cox regression, adjusted for clustering and competing risk analysis (for LPFS) and subsequently tested in multivariate analysis (MVA). The nomogram was built using R statistical software and internally validated using bootstrap resampling.

Results

Patients received RE at a median of 30.9 months (range:3.4-161.7 months) after detection of CLM. Median OS and LPFS were 11.3 (95\%CI, 7.9-15.1) and 4 months (95\% $\mathrm{CI}, 3.3-4.8$ ), respectively. Of the 40 parameters tested, six were independently associated with OS in MVA. These baseline parameters included: number of extrahepatic disease sites $(\mathrm{p}<0.001)$, carcinoembryonic antigen $(\mathrm{CEA})(\mathrm{p}<0.001)$, albumin $(\mathrm{p}=0.005)$, alanine aminotransferase (ALT) level $(p<0.001)$, tumor differentiation level $(p<0.001)$ and sum of the two largest tumor diameters $(\mathrm{p}<0.001)$. One-year OS of patients with total points of $<25 \mathrm{vs}$. $>80$ were $90 \%$ and $10 \%$, respectively. Bootstrap resampling showed good discrimination (optimism corrected $c$-index $=0.745$ ) and calibration (mean absolute prediction error $=0.299$ ) of the nomogram. Only baseline standard uptake value (SUVmax) was significant in MVA for LPFS prediction ( $\mathrm{p}<0.001$, $\mathrm{SHR}=1.06$ ).

Conclusion

The developed nomogram included six pre-RE parameters and provided good prediction of survival post-RE in heavily pretreated patients. Baseline SUVmax was the single significant predictor of LPFS.

\section{INTRODUCTION}

${ }^{90} \mathrm{Y}$ radioembolization (RAE) is an FDA approved liver brachytherapy, recommended through the National Comprehensive Cancer Network (NCCN) and the European Society for Medical Oncology (ESMO) guidelines for the treatment of colorectal cancer liver metastases (CLM) in the salvage setting with liver disease progression while on or after second line chemotherapy with encouraging oncologic outcomes [1-18].

RAE performed at this advanced stage of a terminal disease has the goal of prolonging patient survival with limited if any impact on quality of life. Currently a decision for RAE is recommended through a multidisciplinary discussion [7]. In clinical practice, recommendations for refractory patients are challenging, because this population often presents with advanced tumor load, unfavorable biologic tumor characteristics, comorbidities or poor performance status. It is thus not surprising, that the range of reported outcomes post-RAE is highly variable. Objective response rates varied between $10 \%-48 \%$ when RAE was applied in the third and subsequent chemotherapy regiment setting $[10,17,19,20]$. Thus, careful patient selection is extremely important to minimize the risk of treatment-related complications and unnecessary hospitalization. Current exclusion criteria do not always provide adequate risk stratification or an optimal estimation of patient survival. Poor outcome may be a consequence of more extensive disease, but may also be associated with RAE-induced adverse events. A predictive scoring system can aid a rational decision with estimation of each patient's risk/benefit ratio prior to undergoing RAE.

The aim of this study was to analyze metabolic, anatomic, laboratory, pathologic, primary disease-related and genetic biomarkers, procedure-related factors as wells as pre- and post-RAE therapies that can be associated with liver progression-free and overall survival. In addition, our goal was to create and internally validate a predictive survival nomogram using pre-RAE patient characteristics.

\section{MATERIALS AND METHODS}

Ethical considerations and patient selection

IRB waiver of approval was obtained for this retrospective review of our prospectively created and maintained HIPAA registered and compliant colorectal cancer liver metastases (CLM) RAE database. All patients with CLM treated with ${ }^{90} \mathrm{Y}$ RAE from 9/15/2009 to 3/21/2017 were included. 
Inclusion and exclusion criteria

All patients treated with RAE were eligible for inclusion in the study.

Eligibility criteria for RAE in our practice included: age $\geq 18$ years; Eastern Cooperative Oncology Group (ECOG) performance status 0-2; histologically confirmed primary adenocarcinoma of the colon or rectum; CLM considered unresectable or not amenable to percutaneous ablation; adequate blood cell counts (WBC $>1.5 \times 10^{9} / \mathrm{L}$, platelet count $>50 \times 10^{9} / \mathrm{L}$ ); adequate renal function (creatinine $<1.5 \mathrm{mg} / \mathrm{dL}$ ) and total bilirubin level $\leq 1.5 \mathrm{mg} / \mathrm{dL}$.

Exclusion criteria were: prior hepatic radiotherapy; severe cirrhosis; severe portal hypertension; uncorrectable flow to the gastrointestinal tract and/or $>20 \%$ shunting to the lungs, as determined by technetium-99m labeled macroaggregated albumin

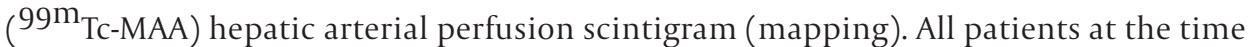
of RAE had liver-dominant disease and were considered candidates for RAE even in the face of oligometastatic (up to 5 sites) extrahepatic disease that was stable or controlled by chemotherapy.

Pre-procedural work - up and angiographic mapping

All patients were evaluated at a clinic visit within 30 days before RAE. Past medical history was reviewed, physical examination was performed and relevant baseline laboratory values were evaluated. Pre-procedural baseline imaging with liver dynamic (ideally triphasic) CT and ${ }^{18}$ F-FDG PET/CT was available within 30 days from RAE for accurate restaging of disease and for calculation of liver and CLM volumes for ${ }^{90} \mathrm{Y}$ dosimetry.

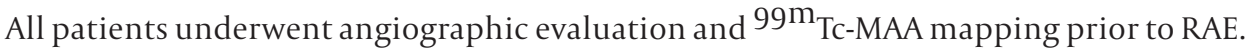
During arteriography, hepatic arterial anatomy and tumor vascular supply were assessed. Extrahepatic vessels with hepatofugal flow within $3 \mathrm{~cm}$ from the desired point of ${ }^{\circ} \mathrm{Y}$ administration were prophylactically coil-embolized to prevent inadvertent delivery of ${ }^{\circ} \mathrm{Y}$ in extrahepatic sites. Once the desired location(s) of RAE administration(s) was determined, a total of $4-5 \mathrm{mCi}$ of ${ }^{99 \mathrm{~m}} \mathrm{Tc}-\mathrm{MAA}$ were injected at desired sites of ${ }^{90} \mathrm{Y}$ arterial infusion and subsequent planar scintigraphy and SPECT/CT imaging were performed to calculate the lung shunt fraction and to detect extrahepatic activity [17].

\section{Radioembolization procedure}

Approximately two weeks after mapping angiography, patients underwent RAE with SIR-Spheres (Sirtex Medical, Sydney, Australia) or glass microspheres (Therasphere; MDS Nordion, Ottawa, Ontario, Canada). Glass-based microspheres were introduced in March
2015 and were specifically used instead of SIR-spheres in patients with higher risk of developing stasis: in the presence of constricted or small caliber arteries as well as in patients with history of prior non-anatomic liver resections or intra-arterial hepatic infusion pump chemotherapy, who presented with tortuous or constricted vessels at mapping.

As of April $2013{ }^{\circ} \mathrm{Y}$ resin microspheres were administered using undiluted contrast medium, which allowed real-time infusion monitoring and resulted in shorter infusion time and reduced fluoroscopy radiation dose as previously described [18].

The total activity of ${ }^{90} \mathrm{Y}$ resin microsphere (in GBq) for each patient was calculated using body surface area (BSA) method with the following formula [21]:

SIR-spheres Activity $=($ treatment liver volume $/$ total liver volume $) *[(B S A-0.2)+$ (fraction of liver with tumor)]

For ${ }^{\circ} \mathrm{Y}$ glass microspheres, total activity of ${ }^{\circ} \mathrm{Y}$ was calculated using the following formula $[7,22]$ :

Therasphere activity $(\mathrm{GBq})=$ Desired dose $(\mathrm{Gy}) \times$ Mass of selected live target lobe/50

In cases where lung shunting was between $10-15 \%$, patients received a $20 \%$ reduction of the calculated radiation dose. Adjustments were not made based on prior treatment history.

CLMs confined to one lobe were treated in one session whereas bilobar disease was treated in two sessions separated by 4- 8 weeks. If the limited extent of disease permitted sublobar microspheres infusions, the patients with bilobar disease were also treated within one session (as long as not more than $50 \%$ of liver parenchyma was exposed to the radiation at each session). Patients with a single hepatic lobe post-resection were treated in lobar or sublobar fashion depending on the extent and location of disease. In general, we aimed to treat as selectively as possible to treat all tumors while sparing uninvolved liver parenchyma.

Study objectives

Primary objectives included:

1) Analysis of liver progression-free survival (LPFS) and factors associated with LPFS. LPFS was defined as the time between the treatment date until disease progression 
or death/last follow- up. It was calculated using competing risk analysis. If there was no liver disease progression and no imaging available within 6 months before death, then the date of last imaging was included in competing risk analysis. If there was no liver disease progression and there was imaging within 6-months before death available, death date was included in the competing risk analysis. LPFS was evaluated using EORTC and RECIST 1.1 criteria, depending on imaging availability. In cases of differing responses between the criteria, the concordance between both modalities imaging findings was used to determine LPFS;

2) Overall survival(OS) and factors associated with it. OS was defined as the time from initial RAE to patient death or last follow-up.

Secondary objectives included:

1) Evaluation of local tumor response and objective response rate (ORR);

2) Assessment of radiologic response within the treated hepatic territory. It was performed using contrast-enhanced CT and ${ }^{18}$ F-FDG PET/CT at 4-8 and 12-16 weeks post-RAE and compared to the pre-RAE scans. Scans at 4-8 weeks were used as the new baseline for subsequent imaging obtained every 2-4 months thereafter. Radiologic response in the liver was assessed by changes in size and metabolic activity using RECIST 1.1 and European Organization for Research and Treatment of Cancer criteria (EORTC). Using EORTC criteria, 25\% threshold was chosen to define response or disease progression, using the sum of up to 5 liver lesions maximum standard uptake values (SUVmax). In patients with two RAE sessions, response was evaluated for each treated region. ORR was defined as the percentage of patients with partial or complete response within 12-16 weeks post-RAE, based on RECIST 1.1 and EORTC criteria.

\section{Prognosticators of LPFS and OS}

Total of 24 factors were analyzed as potential prognosticators of LPFS. They included primary disease-related variables, liver tumor burden, CEA level, genetic mutations (KRAS, BRAF, PI3KCA), prior and post-RAE systemic regimens for metastatic CRC, procedure-related parameters (sphere type, delivered radiation dose and occurrence of stasis), pre-RAE laboratory parameters \{(neutrophil/lymphocyte ratio (NLR) and platelet/lymphocyte ratio (PLR)\} and metabolic tumor uptake parameters \{(SUVmax, SUV peak, SUVmean, metabolic tumor volume (MTV) and total lesion glycolysis (TLG)\} of the most metabolically active tumor.

Forty factors were analyzed as potential prognosticators of OS. In addition to the factors described above, we included the following factors to overall survival analysis: patients' age, pre-RAE liver function laboratory parameters, primary disease-related variables, CEA level, pre-and post-RAE therapies, post-RAE NLR and PLR, disease-free survival, time from liver metastases diagnosis to RAE, LPFS and number of extrahepatic disease sites.

\section{Statistical analysis}

The median follow-up period was calculated for the patients who were alive on last follow-up. Prognosticators of OS and LPFS were selected using univariate Cox regression, adjusted for clustering and competing risk analysis (for LPFS) and subsequently tested in multivariate analysis (MVA). Only pre-treatment easy-to-obtain and well-established parameters were included into MVA as potential predictors of overall survival. All the nomogram factors were included as continuous variables (except for tumor differentiation level) for more accurate estimation of each factor's predictive value. Liver PFS association with OS was assessed using Cox regression analysis, including only the patients, who had liver disease progression.

The nomogram was built using R statistical software and internally validated using bootstrap resampling. Statistical analysis was performed using STATA 12.1 software. A p-value $<0.05$ was considered significant.

\section{RESULTS}

Study population

One hundred and three patients treated with 136 RAE sessions were included in the study. Median follow-up time was 9.02 months (range, 0.7-42.7 months). Sixty-three/103 (61\%) patients were men, 40 (39\%) - women, with a median age of 6o years (range, 2487 years). Seventy-six/103 (74\%) patients had synchronous liver metastases. The median time from diagnosis of CLM to RAE was 30.9 months (range: 3.4-161.7 months).

Eighty-one/103 (79\%) of patients were treated with resin microspheres, 21/103 (20\%) - with glass microspheres, $1 / 103$ (1\%) with both (initially with resin and LTP retreatment with glass microspheres almost 3-years later). Seventy-three/103 (71\%) of patients were treated in one RAE session, 26/103 (25\%)- in two sessions. Seven/103 (7\%) of patients underwent repeat RAE for: (1) LTP in previously treated region (in 3 patients); (2) progression of disease in untreated liver lobe (in 3 patients); (3) additional RAE in the same region due to poor tumor coverage during initial RAE (in 1 patient; partial recanalization of embolized vessel precluded flow redirection to a tumor region during initial RAE). Patient characteristics are described in Table 1. 
Table 1 Patient demographics and disease characteristics.

\begin{tabular}{|c|c|}
\hline Patient demographics and disease characteristics & Number of patients \\
\hline Age (median, range) & 60 (range, $24-87$ years) \\
\hline Gender, M: F & $63: 40$ \\
\hline Synchronous liver disease & $81: 22$ \\
\hline Pathologic evidence of vascular invasion from primary tumor (yes/no/unknown) & 44:33:26 \\
\hline KRAS mutation (yes/no/unknown) & 29:33:16 \\
\hline PIK ${ }_{3}$ CA mutation (yes/no/unknown) & 10: $41: 52$ \\
\hline Surgical resection of primary disease (yes/no/unknown) & 82:20:1 \\
\hline CEA level before RAE (median, range ng/ml) & $55 \cdot 3(1.1-23937.6)$ \\
\hline Time from diagnosis of CRC to diagnosis of CLM (mean, median, range, in months) & $6.3 ; \mathrm{o}($ range $0-64.2)$ \\
\hline Time from diagnosis of CLM to RAE (median, range) & 30.9 (range, 3.4-161.7) \\
\hline Type of microspheres (resin/glass/both) & 81:21:1 \\
\hline Prior liver surgery (yes/no) & 49:54 \\
\hline Prior HAIP chemotherapy (yes/no) & 53:50 \\
\hline Prior systematic chemotherapy ( $\geq 3$ lines vs. $<3$ lines) & 30:73 \\
\hline Prior bevacizumab (yes/no) & $63: 40$ \\
\hline Extent of hepatic replacement by tumor at time of $\operatorname{RAE}(<25 \% ; 225 \%)$ & 89:14 \\
\hline Presence of extrahepatic disease at time of RAE (yes/no) & 74:29 \\
\hline $\begin{array}{l}\text { Number of confirmed metastatic extrahepatic organ systems at time of RAE } \\
(0: 1: 2: 3: 4)\end{array}$ & 29:38:24:9:3 \\
\hline ECOG status at time of RE (Grade o/1/2/unknown) & 33: 26:2: 42 \\
\hline $\begin{array}{l}\text { Distribution of hepatic disease at time of RAE (unilobar/unilobar with one hepatic lobe } \\
\text { left/bilobar) }\end{array}$ & 17: 11: 75 \\
\hline Incidence of stasis in resin microspheres infusions & $39 / 146(27 \%)$ \\
\hline$\%$ of prescribed radiation dose delivered to tumor per procedure & $96 \%$ (range, 19-106\%) \\
\hline Number of RAE sessions per patients (one/two) & 73:30 \\
\hline Post-RAE HAIP* therapy (yes/no) & 18: 85 \\
\hline Number of post-RAE systemic chemotherapy lines (o: 1:2: : : 4: unknown) & 25:39:22:15:1 \\
\hline Post-RAE ablation (yes/no) & 5:98 \\
\hline
\end{tabular}

${ }^{\text {PHAIP-hepatic arterial infusion pump therapy; CEA-carcinoembryonic antigen level; CRC-colorectal cancer; }}$

Local tumor response, objective response rate and liver progression-free survival

On the first imaging post-RAE at 4-8 weeks, $39 \%$ of patients had partial response according to EORTC criteria, whereas $59 \%$ had stable disease according to RECIST 1.1. Objective response rate (complete or partial response) at 12-16 weeks post-RAE was $44 / 84(52 \%)$ by EORTC and 6/94 (6\%) by RECIST 1.1 criteria. Local tumor response at initial $4-8$ weeks postRAE imaging is described in Table 2.

Despite the lack of statistical significance, the patients who developed early/late stasis in at least one infusion during ${ }^{90} \mathrm{Y}$ RAE, had roughly double the risk for local tumor progression on initial scan, defined by RECIST $1.1(\mathrm{p}=0.19, \mathrm{OR}=1.81)$ or EORTC criteria $(\mathrm{p}=0.14, \mathrm{OR}=2.2$.
Table 2 Local tumor response on first imaging after radioembolization (at 4-8 weeks) and at second follow-up (at 12-16 weeks)

\begin{tabular}{lllll}
\hline Response & \multicolumn{3}{c}{ At 4-8weeks } & \multicolumn{1}{c}{ At 12-16 weeks } \\
& EORTC & RECIST 1.1 & EORTC & RECIST 1.1 \\
\hline Complete response (CR) & $5 / 84(6 \%)$ & $1 / 94(1 \%)$ & $3 / 55(5 \%)$ & 0 \\
Partial response (PR) & $33 / 84(39 \%)$ & 0 & $9 / 55(16 \%)$ & $3 / 76(4 \%)$ \\
Stable disease (SD) & $17 / 84(20 \%)$ & $55 / 94(59 \%)$ & $7 / 55(13 \%)$ & $39 / 76(51 \%)$ \\
Progression of disease (POD) & $20 / 84(24 \%)$ & $25 / 94(27 \%)$ & $35 / 55(64 \%)$ & $31 / 76(41 \%)$ \\
Different response in treatment regions: & & & \\
SD+PR & $4 / 84(5 \%)$ & $3 / 94(3 \%)$ & - & - \\
SD+POD & $2 / 84(2 \%)$ & $9 / 94(9 \%)$ & - & $3 / 76(4 \%)$ \\
PR+POD & $2 / 84(2 \%)$ & $1 / 94(\%)$ & - & - \\
SD+CR & $1 / 84(1 \%)$ & 0 & - & - \\
CR+POD & - & - & $1 / 55(2 \%)$ & - \\
Not applicable* & $19 / 103(18 \%)$ & $9 / 103(9 \%)$ & $48 / 103(47 \%)$ & $27 / 103(26 \%)$ \\
\hline
\end{tabular}

disease. ${ }^{*}$ Due

Despite the lack of statistical significance, the patients who had $\geq 96 \%$ of prescribed radiation dose delivered, had $44 \%$ lower risk of local tumor progression at first imaging, compared to patients who received $<96 \%$ of the dose, when LTP was defined by EORTC criteria $(\mathrm{p}=0.32, \mathrm{OR}=0.56)$.

Sphere type was neither associated with local tumor progression on first follow-up, evaluated by RECIST1.1 or EORTC criteria ( $\mathrm{p}=0.99$ for both), nor with liver progressionfree survival $(\mathrm{p}=0.77, \mathrm{SHR}=1.08)$.

Liver progression-free survival (LPFS) and its prognosticators

Median LPFS was 4 months (95\%CI, 3.4-4.9 months). Six-months and 1- year LPFS were 27\% (95\% CI,18-36\%) and 9\% (95\%CI, 3-16\%).

Only increased metabolic tumor parameters (SUVmax, SUVpeak, MTV and TLG) and decreased time from CLM diagnosis to RAE were significantly associated with decreased liver progression-free survival on univariate analysis (Table 3). Due to correlation between metabolic parameters, only two variables (SUVmax and time from CLM diagnosis to RAE) were included in the multivariate analysis (MV). Only SUVmax was significant predictor of LPFS on MV analysis ( $\mathrm{p}=0.003, \mathrm{HR}=1.06,95 \% \mathrm{CI}, 1.02-1.1)$. 
Table 3 Univariate analysis for prognosticators of overall survival (OS) and liver progression-free survival (LPFS) post-radiomebolization (RAE)

\begin{tabular}{|c|c|c|c|c|c|}
\hline Variable group & Variable & $\begin{array}{l}\text { OS, } \\
\text { p-value }\end{array}$ & HR & $\begin{array}{l}\text { LPFS, } \\
\text { p-value }\end{array}$ & SHR \\
\hline $\begin{array}{l}\text { Patient variable } \\
\end{array}$ & Age & 0.23 & 0.76 & - & - \\
\hline \multirow[t]{2}{*}{ Time variable } & Time from diagnosis of CLM to RAE & 0.06 & 0.99 & $0.034^{*}$ & 0.99 \\
\hline & Disease-free interval (from primary Rx to CLM) & 0.07 & 0.99 & & \\
\hline \multirow{6}{*}{$\begin{array}{l}\text { Primary disease- } \\
\text { related variables }\end{array}$} & Primary tumor resection (no/yes) & 0.52 & 0.82 & & \\
\hline & Nodal status of the primary (positive/negative) & 0.77 & 0.33 & - & - \\
\hline & Lymphovascular invasion of primary(yes/no) & $0.03^{*}$ & 1.74 & 0.35 & 0.79 \\
\hline & Tumor differentiation (good/moderate vs. poor) & $0.009^{*}$ & 2.05 & 0.55 & 0.81 \\
\hline & Synchronous vs. metachronous liver metastases & 0.24 & 1.31 & 0.31 & 1.27 \\
\hline & Side of primary (left vs. right; transverse excluded) & 0.33 & 0.77 & 0.45 & 1.25 \\
\hline \multirow{4}{*}{$\begin{array}{l}\text { Disease-related } \\
\text { variables at time } \\
\text { of RAE }\end{array}$} & Liver tumor burden $(\geq 25 \%$ vs. $<25 \%)$ & 0.056 & 1.77 & 0.74 & 1.12 \\
\hline & Number of EHD sites (range, o-4) & $0.03^{*}$ & 1.26 & & - \\
\hline & Sum of largest diameters of two liver lesions $(\mathrm{cm})^{*}$ & $0.001^{*}$ & 1.08 & 0.95 & 1 \\
\hline & CEA level at time of RAE (ng/ml) & $<0.001^{*}$ & 1.0001 & 0.07 & 1.0005 \\
\hline \multirow{3}{*}{$\begin{array}{l}\text { Genetic } \\
\text { mutations }\end{array}$} & KRAS(positive/negative) & 0.16 & 1.45 & 0.15 & 1.48 \\
\hline & PI3KCA(positive/negative) & 0.78 & 1.13 & 0.69 & 0.79 \\
\hline & BRAF(positive/negative) & 0.88 & 0.89 & 0.2 & 1.38 \\
\hline \multirow{3}{*}{$\begin{array}{l}\text { Prior to RAE } \\
\text { variables }\end{array}$} & Prior HAIP (no/yes) & 0.76 & 0.88 & 0.71 & 0.92 \\
\hline & Prior systemic therapy $\left(\geq_{3}\right.$ lines vs. $<3$ lines $)$ & 0.84 & 1.05 & 0.55 & 0.87 \\
\hline & Prior liver surgery & 0.36 & 0.82 & 0.75 & 0.93 \\
\hline \multirow{3}{*}{$\begin{array}{l}\text { RAE-related } \\
\text { variables }\end{array}$} & $\%$ of prescribed radiation dose delivered & 0.43 & 1.004 & 0.6 & 1.003 \\
\hline & Sphere type (resin vs. glass microspheres) & 0.77 & 0.92 & 0.87 & 0.95 \\
\hline & Occurrence of stasis (yes/no) & & & 0.73 & 0.93 \\
\hline \multirow{5}{*}{$\begin{array}{l}\text { Metabolic tumor } \\
\text { uptake } \\
\text { parameters (of } \\
1-5 \text { most FDG-avid } \\
\text { lesions) }\end{array}$} & SUV max (continuous) & $0.028^{*}$ & 1.05 & $<0.001^{*}$ & 1.06 \\
\hline & SUV peak (continuous) & $0.025^{*}$ & 1.06 & $0.001^{*}$ & 1.08 \\
\hline & SUV mean (continuous) & $0.004^{*}$ & 1.13 & 0.41 & 1.03 \\
\hline & MTV (continuous) & $<0.001^{*}$ & 1.001 & $<0.001^{*}$ & 1.0007 \\
\hline & TLG (continuous) & $<0.001^{*}$ & 1.0002 & $0.046^{*}$ & 1.0001 \\
\hline \multirow{7}{*}{$\begin{array}{l}\text { Pre-RAE laboratory } \\
\text { parameters }\end{array}$} & Pre-RAE NLR & $0.02^{*}$ & 1.05 & 0.4 & 1.02 \\
\hline & Pre-RAE PLR & $0.001^{*}$ & 1.002 & 0.12 & 1.001 \\
\hline & Albumin & $<0.001^{*}$ & 0.36 & - & - \\
\hline & Total bilirubin & 0.4 & 1.26 & - & - \\
\hline & AST (aspartate aminotransferase) & $<0.001^{*}$ & 1.02 & - & - \\
\hline & ALT (alanine aminotransferase) & $0.003^{*}$ & 1.01 & & \\
\hline & ALP(alkaline phosphatase) & $0.001^{*}$ & 1.002 & - & - \\
\hline \multirow{7}{*}{$\begin{array}{l}\text { Post-RAE } \\
\text { laboratory } \\
\text { parameters| } \\
\text { therapies }\end{array}$} & Post-RAE NLR (neutrophil//ymphocyteratio) & 0.077 & 1.04 & & - \\
\hline & Post-RAE PLR (platelet/lymphocyte ratio) & 0.6 & 1.0003 & - & - \\
\hline & Post-RAE HAIP (hepatic artery infusion pump) & $0.001^{*}$ & 0.42 & & - \\
\hline & Post-RAEablation & $0.029^{*}$ & 0.35 & - & - \\
\hline & Post-RAE chemotherapy lines ( $\leq 1$ vs 2-4) & $<0.001^{*}$ & 0.48 & - & - \\
\hline & Post-RAE bevacizumab & $0.001^{*}$ & 0.48 & - & - \\
\hline & Liver progression-free survival (in months) & $0.002^{*}$ & 0.9 & - & - \\
\hline
\end{tabular}

Overall survival

Median OS was 11.3 months (95\% CI 7.9 - 15.1 months). Six months, 1-, 2- and 3 - year OS was $75 \%$ (95\%CI, 64-81\%), $46 \%$ (95\%CI, 35-55\%), 18\% (95\%CI, 11-27\%) and 15\% (95\%CI, 8-24\%), respectively. There were no deaths within 30 days post-RAE.

Total of 11/103 (11\%) patients died within 3 months post- RAE. None of these deaths were considered directly or solely related to the RAE: 7/11 (64\%) of the patients had evidence of liver disease progression; 8/11 (73\%) patients also developed conditions, that resulted in significant morbidity and were at least partially related to RAE, but did not lead to subsequent death. Analysis of deaths within 3-months post-RAE is presented in Table 4.

Factors associated with overall survival: creation of predictive nomogram

Twenty-one/40 factors were associated with overall survival post-RAE in univariate analysis (Table 3). Considering the number of deaths during the study period $\{87 / 103$ (84\%)\}, only 8 factors with low correlation between each other could be included in MV analysis.

Six/8 pre-RAE parameters included into MV analysis were significantly associated with OS and were subsequently used to build an overall survival prediction nomogram (Table 5). These factors included: (1) baseline CEA level (2) baseline ALT level (3) albumin level (4) sum of sizes of two largest CLM diameters in the treatment region (5) number of extrahepatic disease sites and (6) tumor differentiation level (Figure 1).

One patient had CEA level $>20000 \mathrm{ng} / \mathrm{ml}$ and was considered as an outlier in our study, that contributed to very high CEA values in the nomogram. It was excluded from internal validation as it was adversely affecting model fit and evaluation.

Each MV analysis factor was assigned points relative to each hazard ratio. The nomogram assigned the probability of 1-year survival by summing the point-scale scores for each variable. The total score projected on the bottom scale indicated the probability of 1-year survival. One-year OS of patients with total points $<25$ indicated 90\% chance of 1-year survival, whereas total sum of points of $>80$ estimated $10 \%$ chance of 1 -year survival. Bootstrap resampling showed good discrimination (optimism corrected c- index $=0.745$ ) and calibration (mean absolute prediction error=0.299) of the nomogram.

Complications

The incidence of Grade 1-2 toxicities was 44/102 (43\%) (Table 6). The incidence of Grade 3-4 toxicities was $14 / 102$ (14\%) (Table 6). Two patients in our study developed hepatic 
venous occlusion post-RAE: Budd-Chiari syndrome in one patient and right hepatic vein, inferior vena cava thrombus and bilateral pulmonary embolism in second patient with lung disease progression.

Table 4 Overview of post-procedure course of patients who expired within 3 months postradioembolization

\begin{tabular}{|c|c|c|}
\hline $\begin{array}{l}\text { Baseline patient } \\
\text { characteristics }\end{array}$ & Post-RAE course/potential reason of death & Death time \\
\hline $\begin{array}{l}\text { Hx of HAIP; } \\
>25 \% \mathrm{~TB}, \\
\text { bilobar liver disease; CEA }= \\
766 \mathrm{ng} / \mathrm{ml} \text {; } \mathrm{EHD} \text { sites }=2\end{array}$ & $\begin{array}{l}\text { RAE-related major toxicity (grade 3-4 chest pain, SOB, pleural effusion } \\
\text { in face of stable lung metastases one-month post-RAE); managed } \\
\text { conservatively. Death cancer-related, likely due liver failure with evidence } \\
\text { of liver POD (one-month post-RAE) and EHD POD (lungs, lymph nodes) }\end{array}$ & $\begin{array}{l}1.93 \\
\text { months }\end{array}$ \\
\hline $\begin{array}{l}\text { Hx of HAIP; bilobar liver } \\
\text { disease, } \mathrm{CEA}=1357 \mathrm{ng} / \mathrm{ml} \text { EHD } \\
\text { sites }=4\end{array}$ & $\begin{array}{l}\text { RAE-related major toxicity: grade } 4 \text { SOB (acute hypersensitivity to } \\
\text { steroids post-procedure), resolved. Cancer-related death in face of } \\
\text { EHD POD one- month post-RAE and liver POD on first follow-up; Hx of } \\
\text { hemochromatosis }\end{array}$ & 1.53 months \\
\hline $\begin{array}{l}\text { Hx of liver } \mathrm{Rx} \text { and HAIP; } \\
>25 \% \\
\text { TB, bilobar liver disease, } \\
\text { CEA }=228 \mathrm{ng} / \mathrm{ml} \text { EHD sites }=2\end{array}$ & $\begin{array}{l}\text { Cancer-related death, likely due liver failure (ascites/grade } 3 \\
\text { hyperbilirubinemia/anasarca) in face of liver POD (which precluded } \\
2 \text { nd RAE session), lung POD and bilateral pulmonary embolism/ } \\
\text { pleural effusion/IVC and RHV thrombi 1-month post-RAE (treated with } \\
\text { anticoagulation) }\end{array}$ & 2.93 months \\
\hline $\begin{array}{l}\text { Hx of HAIP; bilobar liver } \\
\text { disease, CEA=218ng/ml }\end{array}$ & $\begin{array}{l}\text { Unknown death reason; likely liver failure in face of RFA 1-month post- } \\
\text { RAE with grade } 4 \text { hyperbilirubinemia/fever) }\end{array}$ & 2.27 months \\
\hline $\begin{array}{l}\text { Hx of liver Rx and HAIP; EHD } \\
\text { sites=1; ECOG=1 }\end{array}$ & $\begin{array}{l}\text { RAE contributed to fatal outcome (dramatic increased of LFTs right post- } \\
\text { RAE); also, liver POD, secondary biliary obstruction, coagulopathy, } \\
\text { colitis, duodenal stenosis, required stenting, HBV }\end{array}$ & 1.27 months \\
\hline $\begin{array}{l}\text { Hx of HAIP, mitomycin, } \geq 3 \\
\text { chemotherapy lines, bilobar } \\
\text { disease, } C E A=4713 n g / m l\end{array}$ & $\begin{array}{l}\text { Death reason unknown, likely liver failure in patient with history of } \\
\text { hemochromatosis (2-months post-RAE admitted for abdominal pain in } \\
\text { outside hospital with biliary stricture, cirrhosis, ascites) }\end{array}$ & 2.9 months \\
\hline $\begin{array}{l}\text { Hx of liver Rx, HAII, } \geq 3 \\
\text { chemotherapy lines, bilobar } \\
\text { disease, EHD sites }=2\end{array}$ & $\begin{array}{l}\text { Post-RAE: ECOG decline (from o to } 3,5 \text {-days post-RAE). Could not proceed } \\
\text { with planned nd }{ }_{\text {RAE session due grade } 3 \text { hyperbilirubinemia in face of }} \\
\text { liver POD on first follow-up }\end{array}$ & 2.47 months \\
\hline $\begin{array}{l}\text { Hx of HAIP, } \geq 3 \text { chemotherapy } \\
\text { lines, bilobar disease, EHD } \\
\text { sites }=2\end{array}$ & $\begin{array}{l}\text { Post-RAE: severe abdominal, back pain/anorexia/ SOB/chest pain/ phlegm } \\
\text { 1- week post-RAE;11 days post-RAE admitted for pneumonia, recovered. } \\
\text { Death cancer-related (although no records within } 3 \text { weeks before death) }\end{array}$ & 1.83 months \\
\hline $\begin{array}{l}\text { Bilobar disease, } \mathrm{CEA}=130 \\
\text { ng/ml, } \\
\text { EHD sites }=2, \mathrm{ECOG}=1\end{array}$ & $\begin{array}{l}\text { Death cancer-related in face of untreated liver POD, peritoneal } \\
\text { carcinomatosis and not-RAE related adverse event }(6 \text { weeks post-RAE } \\
\text { obstruction due stomach bleed (patient was on Xarelto) requiring } \\
\text { transfusion;significantECOG decline }\end{array}$ & 1.77 months \\
\hline $\begin{array}{l}\text { Bilobar disease, EHD sites }=2 \\
\mathrm{ECOG}=1\end{array}$ & $\begin{array}{l}\text { Grade 3-4 toxicity: Post-embolization syndrome. Six weeks post-RAE } \\
\text { urosepsis, } 2 \text { months post-RAE: brain radiotherapy for brain metastases, } \\
\text { then altered mental status/ hypoxia due fluid overload, leptomeningeal } \\
\text { metastases }\end{array}$ & 2.13 months \\
\hline $\begin{array}{l}\text { Hx of HAIP; bilobar disease, } \\
\text { CEA }=404 \mathrm{ng} / \mathrm{ml} \text {; EHD sites }=1\end{array}$ & No report of RAE-related toxicity and other adverse events after RAE & 2.3 months \\
\hline
\end{tabular}

Table 5 Multivariable analysis of factors associated with overal survival post-radiomebolization

\section{Variable included to MVA}

Tumor differentiation level (good/moderate vs. poor)

Sum of largest diameters of two liver target lesions

Number of EHD sites (range, $0-4$ )

CEAlevel at time of RAE $(\mathrm{ng} / \mathrm{ml})$

Albumin

ALT (alanine aminotransferase)

Lymphovascular invasion on pathology of primary

Pre-RAE NLR (neutrophil/lymphocyte ratio) $\begin{array}{lll}\text { p-value } & \text { HR } & 95 \% \mathrm{CI}\end{array}$

$<0.0001 \quad 5.3 \quad 2.6-10.7$

$<0.0001 \quad 1.15 \quad 1.09-1.21$

$<0.001 \quad 1.00009 \quad 1.00006-1.000$

$\begin{array}{lll}0.005 & 0.46 & 0.27-0.79\end{array}$

$<0.0001 \quad 1.02 \quad{ }_{101-1.03}$

$>0.05 \quad$ Excluded by stepwise forward selection

$>0.05 \quad$ Excluded by stepwise forward selection $\begin{array}{lll}0.016 & 1.41 & 1.07-1.87\end{array}$

EHD-extrahepatic disease: CFA-carcinoembryonic antigen level, RAE-radioembolization

Table 6 Post-radiomebolization toxicities

\begin{tabular}{|c|c|c|}
\hline \multicolumn{3}{|c|}{ Grade 1-2 toxicities } \\
\hline 1. & Fatigue/weakness & $43 / 102(42 \%)$ \\
\hline 2. & Abdominal pain/distension & $23 / 102(23 \%)$ \\
\hline 3. & Constipation/diarrhea & $14 / 102(14 \%)$ \\
\hline 4. & Fever & $11 / 102(11 \%)$ \\
\hline 5. & Change in appetite/weight changes & $11 / 102(11 \%)$ \\
\hline 6. & Nausea & $10 / 102(10 \%)$ \\
\hline 7. & Light colored stool/dark urine & $10 / 102(10 \%)$ \\
\hline 8. & Shortness of breath & $10 / 102(10 \%)$ \\
\hline 9 . & Ascites & $5 / 102(5 \%)$ \\
\hline 10. & Vomiting & $4 / 102(4 \%)$ \\
\hline 11. & Shoulder/back pain & $4 / 102(4 \%)$ \\
\hline 12. & Hiccups & $1 / 102(1 \%)$ \\
\hline 13. & Portal hypertension & $1 / 102(1 \%)$ \\
\hline 14. & Portal hypertension/ascites in patient with prior oxaliplatin administration & $1 / 102(1 \%)$ \\
\hline TOTAL & & $44 / 102(43 \%)$ \\
\hline \multicolumn{3}{|c|}{ Grade $3-4$ toxicities } \\
\hline 1. & Post-radioembolization syndrome (at 1-9o days) & $5 / 102(5 \%)$ \\
\hline 2. & Grade 3 abdominal pain/distension (at 10-12 days) & $3 / 102(3 \%)$ \\
\hline 3. & Grade 3 fevers (at 3 days) & $1 / 102(1 \%)$ \\
\hline 4. & Acute liver failure/ failure to thrive/ grade 3 hyperbilirubinemia (at 14 days) & $1 / 102(1 \%)$ \\
\hline 5. & Grade 3 dyspnea (at zo days) & $1 / 102(1 \%)$ \\
\hline 6. & $\begin{array}{l}\text { Biliary obstruction + Budd-Chiari syndrome+ stop chemotherapy for } 6 \text { weeks } \\
\text { (at 1-day/6o-days) }\end{array}$ & $1 / 102(1 \%)$ \\
\hline 7. & $\begin{array}{l}\text { Biloma requiring multiple drainages, liver abscess, E. Faecium sepsis; patient with liver } \\
\text { POD (at } 5 \text { days) }\end{array}$ & $1 / 102(1 \%)$ \\
\hline 8. & Post-mapping brain infarction due anticoagulation break in patient with history of atrial & 1/102(1\%) \\
\hline
\end{tabular}

8. Post-mapping brain infarction due anticoagulation break in patient with history of atrial
fibrillation. Post-procedure hypertension, recurrent atrial fibrillation, abdominal pain, hypotension, fever, required overnight admission (at 1 day)

$14 / 102(14 \%)$

One patient was lost to follow-up post procedure; Multiple complications possible per patient; POD-progression of 
Points

Size

EHD

CEA

Albumin

Differentiation

Total Points

1 Year Sunvival

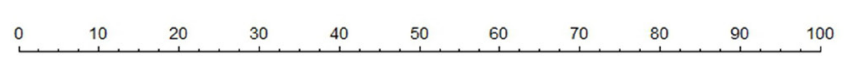

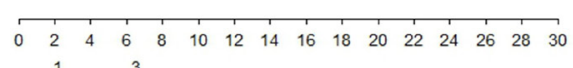

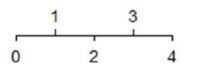

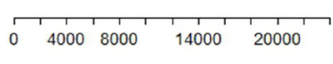

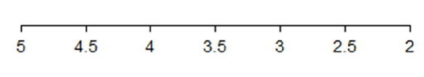

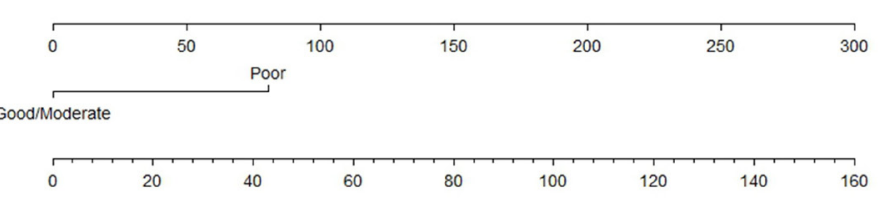

$\begin{array}{llll}0.9 & 0.8 & 0.7 & 0.60 .50 .40 .30 .2 \\ 0.1\end{array}$
Figure 1 Nomogram for overall survival prediction after radioembolization of colorectal cancer liver metastases. Size - sum of largest diameters of two largest liver lesions in intended to-treat-regio; EHD- number of extrahepatic disease sites; CEA-carcinoembryonic antigen level (ng/ml); Alt - alanine aminotransferase (U/L); differentiation- tumor differentiation level.

Example 1 of nomogram points calculation. The patient presented for radioembolization with following parameters: sum of two largest liver lesions diameters of $11.1 \mathrm{~cm}$, two sites of extrahepatic disease, CEA 765.6 $\mathrm{ng} / \mathrm{ml}$, albumin of $2.3 \mathrm{~g} / \mathrm{dl}$, ALT of $27 \mathrm{U} / \mathrm{L}$ and poor tumor differentiation level. By drawing a straight line from the $11.1 \mathrm{~cm}$ point on the Size axis of the nomogram to the Points axis we find that a lesion of $11.1 \mathrm{~cm}$ corresponded to 25 points. Repeating the same for the other variables, we got 10 points for two sites of extrahepatic disease, 1 point for CEA of $765.6 \mathrm{ng} / \mathrm{ml}, 45$ points for albumin of $2.3 \mathrm{~g} / \mathrm{dl}$ points, 9 points for ALT of $27 \mathrm{U} / \mathrm{L}$ and 27 points for poor tumor differentiation. Sum of these points was 117 and again drawing a straight line from 117 on the Total Points axis to the 1 Year Survival prediction axis we obtained $<10 \%$ as the predicted probability of 1 -year survival for this patient. The patient had actual overall survival of 1.9 months.

Example 2 of nomogram points calculation. The patient had following baseline characteristics: sum of two largest liver lesions diameters of $1.8 \mathrm{~cm}$, no extrahepatic disease, CEA $2.9 \mathrm{ng} / \mathrm{ml}$, albumin of $4.5 \mathrm{~g} /$ $\mathrm{dl}$, ALT of $34 \mathrm{U} / \mathrm{L}$ and moderate tumor differentiation level. By drawing a straight line from the $1.8 \mathrm{~cm}$ point on the Size axis of the nomogram to the Points axis we find that a sum of lesions diameters of $1.8 \mathrm{~cm}$ corresponded to 4 points. Repeating the same for the other variables, we got o points for no extrahepatic disease, o points for CEA of $2.9 \mathrm{ng} / \mathrm{ml}$, 9 points for albumin of $4.5 \mathrm{~g} / \mathrm{dl}$ points, 12 points for ALT of $34 \mathrm{U} / \mathrm{L}$ and o points for moderate tumor differentiation. Sum of these points was 25 and again drawing a straight line from 25 on the Total Points axis to the 1 Year Survival prediction axis we obtained 90\% as the predicted probability of 1-year survival for this patient. The patient had actual overall survival of 60.3 months.

\section{DISCUSSION}

This work provided an extensive, comprehensive analysis of imaging, laboratory, pathologic, genetic, primary-disease-related biomarkers, and procedure - related factors as well as prior and post-RAE therapies, associated with overall and liver progressionfree survival following RAE of CLMs.

Overall survival prediction nomogram

The developed nomogram included six easy-to-obtain pre-RAE parameters and provided good prediction of patient survival post-RAE, based on internal bootstrap validation result: nomogram showed good discrimination ( $c$-index $=0.745)$ and calibration. Oneyear overall survival of patients with total nomogram points of $<25 \mathrm{vs}$. $>80$ was $90 \%$ and $10 \%$, respectively.

Existing overall survival prediction nomogram, created by Fendler et al [23] potentially suffered from selection bias due to the inclusion of patients with very limited extrahepatic metastases and did not demonstrate any association of extrahepatic metastases with survival, contradictory to the reported negative impact of extrahepatic disease on patient survival post-RAE [24-26]. Overall $73 \%$ of this study patients had extrahepatic metastases at the time of RAE. Recent multicenter randomized phase 3 trials (FOXFIRE, SIRFLOX, and FOXFIRE-Global) showed no survival benefit when combining ${ }^{90} \mathrm{Y}$ radioembolization with first-line chemotherapy [13]. According to existing evidence, patients will continue to present for RAE at latest stages of disease, especially when they progress in the liver while receiving second, third or subsequent chemotherapy regimens. RAE is recommended in the chemo-refractory or salvage setting [1]. It is therefore important to identify and describe predictive factors in these settings. An overall survival prognostic score, developed by Damm et al, included heavily pretreated patients, with a median baseline Karnofsky index of $80 \%$ and liver tumor load of $20 \%$. The overall survival post-RAE was 6.7 months. This suggests that radioembolization was a relatively aggressive therapy for this specific compromised population [27]. In our study, only $3 \%$ of patients had ECOG grade 2 performance status and only $14 \%$ had $\geq 25 \%$ baseline liver involvement by tumor.

All increased metabolic tumor uptake parameters of most metabolically active tumor (SUVmax, SUVpeak, SUVmean, MTV, TLG) within the intended-to-treat region were significantly associated with decreased overall survival. Recent studies confirmed that ${ }^{18} \mathrm{~F}$-FDG PET/CT is useful to evaluate treatment response and it is an emerging prognostic tool in patients with CLM undergoing RAE, with semi-quantitative factors (as MTV and 
TLG) correlating with survival better than RECIST criteria $[28,29]$. We therefore advocate metabolic imaging to be always performed before RAE. Prior investigations described anatomic biomarkers, such as CLM size, as predictor of decreased survival post-RAE [23, 30-32]. Measuring the sum of two largest lesion diameters is also important for RECIST 1.1 evaluations and was part of our nomogram. Both anatomic (lesion size) or metabolic tumor parameters were statistically significant predictors of overall survival in this study. However, both factors could not be included into the nomogram, because lesion size generally correlates with metabolic uptake. Thus, due to the fact, that ${ }^{18}$ F- FDG PET/ $\mathrm{CT}$ is not always available in other institutions for treatment planning, we chose to include anatomic parameters into the nomogram.

ALT (SGPT, alanine aminotransferase), unlike other routine liver panel enzymes, is predominantly found in the liver and released into the bloodstream as the result of liver injury, thus serves as a fairly specific indicator of liver damage. ALT was the most predictive nomogram factor of decreased survival: ALT level $>250 \mathrm{U} / \mathrm{L}$ alone corresponded to $>80$ nomogram points, distinguishing patients with $>90 \%$ risk for death within 1-year after RAE. Other liver enzymes - aspartate aminotransferase and alkaline phosphatase - were also found to be associated with post-RAE survival. Since we could only include a limited number of factors in the MV analysis and the latter factors are less hepatic functionspecific biomarkers compared to ALT, they were not included. Transaminase toxicity was predictive of survival post-RAE in prior studies [23,33], thus it should be emphasized as an important patient selection criterion in patients with CLM. Total bilirubin level was not predictive of survival in our study, most likely due to the strict inclusion criteria of RAE in our center. Only patients with total bilirubin $\leq 1.5 \mathrm{mg} / \mathrm{dl}$ were eligible for RAE. Commonly, patients with bilirubin $<2 \mathrm{mg} / \mathrm{dl}$ are considered eligible for RAE [34].

CEA level differed dramatically in this cohort, with a median of $52 \mathrm{ng} / \mathrm{ml}$, ranging from normal level to $>20000 \mathrm{ng} / \mathrm{ml}$, considered as an outlier in our study, that contributed to very high CEA values in the nomogram. Increased CEA level has been associated with poorer survival post-RAE of CLM in multiple studies [4,28,33,35], however, this is influenced by multiple factors, including tumor differentiation level, liver function and the side of primary tumor [35]

The overall survival predictors in this study are similar to a recent study, where higher CEA and AST level, NLR >5, extrahepatic disease, and larger volume of liver metastases were independently associated with increased risk of death on multivariate analysis [36].

Median overall survival in this cohort was 11.3 months post-RAE. This compares favorably to large retrospective studies with reported median survival of 7.2-10.6 months after radioembolization of CLM, mostly in the salvage setting [37-39]. It should be noted, that RAE in this cohort was administered at a median of 30.9 months from the initial diagnosis of CLM. This reflects that most patients were heavily pre-treated and received multiple prior liver-directed therapies, including resection, HAIP and ablation. Pre-RAE therapies were not associated with overall survival, although, the fact, that most patients received loco-regional therapies pre-RAE might have influenced that. The actual liver function and parameters indicating underlying hepatic toxicity (albumin and ALT level), the metastatic disease volume (liver tumor(s) size, extrahepatic disease extent and CEA level) as well as tumor differentiation were significant predictors of post-RAE overall survival.

All analyzed post - RAE therapies (increased number of systemic chemotherapy lines, HAIP, ablation and bevacizumab) were associated with increased overall survival postRAE. This result is concordant to prior studies, where RAE did not prohibit re-initiation of systemic chemotherapy, hepatic arterial infusion pump therapy or ablation for CLM that persisted or progressed after initial response to RAE [4, 9, 12, 40-42]. This also demonstrated that overall survival post-RAE cannot be attributed solely to RAE, but rather to the overall management of the disease in the salvage setting. Post-RAE therapies were not included in the 1-year overall survival prediction nomogram. This was necessary to allow us to reach our goal of patient stratification before RAE in this setting.

Patients with KRAS or PI3KCA mutations and left-sided primary tumors showed decreased overall survival following RAE in our study, however, the difference was not statistically significant. Patients with BRAF mutation and right-sided primary tumor showed increased post-RAE survival, but again-not statistically significantly.

Predictors of local tumor progression and liver progression-free survival

Objective response rate of $52 \%$ in this study compares favorably with published data of 10-48\% response rates, depending on whether the patients received RAE with second line chemotherapy or at the salvage setting $[10,17,19,20,43]$. Baseline SUVmax was the only significant predictor of liver progression-free survival on MV analysis. There was no significant difference in liver progression-free survival for those, who received resin or glass microspheres. Although the incidence of stasis, resulting in suboptimal radiation dose delivery to tumor increased the risk of local tumor progression, it did not reach statistical significance. PI 3 KCA mutation was not a significant predictor of LPFS in this cohort, unlike in prior study [44]. This could be related to difference in methodology between studies (response evaluation criteria, evaluation of local tumor progression, 
rather than LPFS and analysis of different $\mathrm{PI}_{3} \mathrm{~K}$ mutations, associated with gain of function).

\section{Safety of ${ }^{90}$ Y radioembolization}

RAE had acceptable safety profile in this series with incidence of grade 3-4 toxicities of $14 \%$, median overall survival of 11.3 months, no death within 30 days post-procedure and no RAE-related death within 3-months post-treatment.

Two patients in this study developed liver venous occlusion post-RAE: Budd-Chiari syndrome in one patient and right hepatic vein, inferior vena cava thrombus and bilateral pulmonary embolism in second patient. Radiation therapy was shown to cause endothelial activation with subsequent pro-thrombotic response and tissue inflammation with subsequent vein external compression, stasis and thrombus formation [45]. However, the number of patients with these complications was too small to draw meaningful conclusions regarding liver venous occlusion association with RAE.

\section{Study limitations}

Study limitations included the single center retrospective study design with its inherited limitations and a relatively short follow-up time. Also, there was a lack of external nomogram validation that preferably would include prospectively recruited patients at multiple centers. Finally, actual radiation dose delivered to the tumor could not be measured in this study due to technical limitations, thus it could not be analyzed as a prognosticator of RAE.

\section{CONCLUSION}

The developed nomogram included six pre-RAE parameters and provided good prediction of patient survival post-RAE. Baseline SUVmax was the single significant predictor of liver progression-free survival on multivariate analysis.

\section{REFERENCES}

1. Benson AB, 3rd, Venook AP, Cederquist L, Chan E, Chen YJ, Cooper HS, et al. Colon Cancer, Version 1.2017, NCCN Clinical Practice Guidelines in Oncology. J Natl Compr Canc Netw. 2017;15(3):37098.

2. Khajornjiraphan N, Thu NA, Chow PK. Yttrium-go microspheres: a review of its emerging clinical indications. Liver Cancer. 2015;4(1):6-15.

3. Murray D, McEwan AJ. Radiobiology of systemic radiation therapy. Cancer Biother Radiopharm. 2007;22(1):1-23.

4. Sofocleous CT, Violari EG, Sotirchos VS, Shady W, Gonen M, Pandit-Taskar N, et al. Radioembolization as a Salvage Therapy for Heavily Pretreated Patients With Colorectal Cancer Liver Metastases: Factors That Affect Outcomes. Clin Colorectal Cancer. 2015;14(4):296-305.

5. Campbell AM, Bailey IH, Burton MA. Analysis of the distribution of intra-arterial microspheres in human liver following hepatic yttrium-9o microsphere therapy. Phys Med Biol. 2000;45(4):1023-33.

6. Van Cutsem E, Cervantes A, Adam R, Sobrero A, Van Krieken JH, Aderka D, et al. ESMO consensus guidelines for the management of patients with metastatic colorectal cancer. Ann Oncol. 2016;27(8):1386-422.

7. Kennedy A, Nag S, Salem R, Murthy R, McEwan AJ, Nutting C, et al. Recommendations for radioembolization of hepatic malignancies using yttrium-go microsphere brachytherapy: a consensus panel report from the radioembolization brachytherapy oncology consortium. Int J Radiat Oncol Biol Phys. 2007;68(1):13-23.

8. Sotirchos VS, Petre EN, Brown KT, Brody LA, D’Angelica MI, DeMatteo RP, et al. Safe and
Successful Yttrium-go Resin Microsphere Radioembolization in a Heavily Pretreated Patient with Chemorefractory Colorectal Liver Metastases after Biliary Stent Placement above the Papilla. Case Reports Hepatol. 2014;2014:921406.

9. Sofocleous CT, Garcia AR, Pandit-Taskar N, Do KG, Brody LA, Petre EN, et al. Phase I trial of selective internal radiation therapy for chemorefractory colorectal cancer liver metastases progressing after hepatic arterial pump and systemic chemotherapy. Clin Colorectal Cancer. 2014;13(1):27-36.

10. Hendlisz A, Van den Eynde M, Peeters M, Maleux G, Lambert B, Vannoote J, et al. Phase III trial comparing protracted intravenous fluorouracil infusion alone or with yttrium-9o resin microspheres radioembolization for liver-limited metastatic colorectal cancer refractory to standard chemotherapy.J J Clin Oncol. 2010;28(23):3687-94.

11. Van Hazel G, Blackwell A, Anderson J, Price D, Moroz P, Bower G, et al. Randomised phase 2 trial of SIR-Spheres plus fluorouracil/leucovorin chemotherapy versus fluorouracil/leucovorin chemotherapy alone in advanced colorectal cancer. J Surg Oncol. 2004;88(2):78-85.

12. Gray B, Van Hazel G, Hope M, Burton M, Moroz P, Anderson J, et al. Randomised trial of SIRSpheres plus chemotherapy vs. chemotherapy alone for treating patients with liver metastases from primary large bowel cancer. Ann Oncol. 2001;12(12):1711-20.

13. Wasan HS, Gibbs P, Sharma NK, Taieb J, Heinemann V, Ricke J, et al. First-line selective internal radiotherapy plus chemotherapy versus chemotherapy alone in patients with liver metastases from colorectal cancer (FOXFIRE, 
SIRFLOX, and FOXFIRE-Global): a combined analysis of three multicentre, randomised, phase 3 trials. Lancet Oncol. 2017;18(9):1159-71.

14. Kennedy AS, Nutting C, Coldwell D, Gaiser J, Drachenberg C. Pathologic response and microdosimetry of (90)Y microspheres in man: review of four explanted whole livers. Int J Radiat Oncol Biol Phys. 2004;60(5):1552-63.

15. Sharma RA, Van Hazel GA, Morgan B, Berry DP, Blanshard K, Price D, et al. Radioembolization of liver metastases from colorectal cancer using yttrium-go microspheres with concomitant systemic oxaliplatin, fluorouracil, and leucovorin chemotherapy.J Clin Oncol. 2007;25(9):1099-106.

16. Vente MA, Wondergem $M$, van der Tweel I, van den Bosch MA, Zonnenberg BA, Lam MG, et al. Yttrium-go microsphere radioembolization for the treatment of liver malignancies: a structured metaanalysis. Eur Radiol. 2009;19(4):951-9.

17. Cosimelli M, Golfieri R, Cagol PP, Carpanese L,Sciuto R, Maini CL, et al. Multi-centre phase II clinical trial of yttrium-go resin microspheres alone in unresectable, chemotherapy refractory colorectal liver metastases. Br J Cancer. 2010;103(3):324-31.

18. Kurilova I, Beets-Tan RGH, Ulaner GA, Boas FE, Petre EN, Yarmohammadi H, et al. (go)Y Resin Microspheres Radioembolization for Colon Cancer Liver Metastases Using Full-Strength Contrast Material. Cardiovasc Intervent Radiol. 2018

19. Bester L, Meteling B, Pocock N, Saxena A, Chua TC, Morris DL. Radioembolisation with Yttrium-90 microspheres: an effective treatment modality for unresectable liver metastases. J Med Imaging Radiat Oncol. 2013:57(1):72-80.

20. Seidensticker R, Denecke T, Kraus P, Seidensticke M, Mohnike K, Fahlke J, et al. Matched-pai comparison of radioembolization plus best supportive care versus best supportive care alone for chemotherapy refractory liver-dominant colorectal metastases. Cardiovasc Intervent Radiol. 2012;35(5):1066-73.

21. SIR-Spheres® Y-9o resin microspheres Package

22. TheraSphere Yttrium-9o Microspheres Instructions for Use.

23. Fendler WP, Ilhan H, Paprottka PM, Jakobs TF, Heinemann V, Bartenstein P, et al. Nomogram including pretherapeutic parameters for prediction of survival after SIRT of hepatic metastases from colorectal cancer. Eur Radiol. 2015;25(9):2693-700.

24. Tohme S, Sukato D, Nace GW, Zajko A, Amesur N, Orons $\mathrm{P}$, et al. Survival and tolerability of liver radioembolization: a comparison of elderly and younger patients with metastatic colorectal cancer. HPB (Oxford) . 2014;:16(12):1110-6.

25. Rougier P, Milan C, Lazorthes F, Fourtanier G, Partensky C, Baumel H, et al. Prospective study of prognostic factors in patients with unresected hepatic metastases from colorectal cancer. Fondation Francaise de Cancerologie Digestive. Br Surg. 1995;82(10):1397-400

26. Stangl R, Altendorf-Hofmann A, Charnley RM, Scheele J. Factors influencing the natural history of colorectal liver metastases. Lancet. 1994;343(8910):1405-10.

27. Damm R, Seidensticker R, Ulrich G, Breier L, Steffen G, Seidensticker M, et al. Ygo Radioembolization in chemo-refractory metastastic, liver dominant colorectal cancer patients: outcome assessment applying a predictive scoring system. BMC Cancer. 2016;16:509.

28. Fendler WP, Philippe Tiega DB, Ilhan H, Paprottka PM, Heinemann V, Jakobs TF, et al. Validation of several SUV-based parameters derived from 18F-FDG PET for prediction of survival after SIRT of hepatic metastases from colorectal cancer. J Nucl Med. 2013;54 (8):1202-8.

29. Shady W, Kishore S, Gavane S, Do RK, Osborne JR, Ulaner GA, et al. Metabolic tumor volume and total lesion glycolysis on FDG-PET/CT can predict overall survival after $(90) Y$ radioembolization of colorectal liver metastases: A comparison with SUVmax, SUVpeak, and RECIST 1.o. Eur J Radiol. 2016;85(6):1224-31.

30. Dunfee BL, Riaz A, Lewandowski RJ, Ibrahim S, Mulcahy MF, Ryu RK, et al. Yttrium-go radioembolization for liver malignancies: prognostic factors associated with survival. Journal of vascular and interventional radiology : JVIR 2010;21(1):90-5.

31. Schonewolf CA, Patel B, Gensure RH, Narra V, Haffty BG, Nosher JL, et al. Patterns of failure in colorecta patients with liver metastases after yttrium-90 radioembolization. Am J Clin Oncol. 2014;37(3):234-

32. Abbott AM, Kim R, Hoffe SE, Arslan B, Biebel B, Choi ] et al. Outcomes of Therasphere Radioembolization for Colorectal Metastases. Clin Colorectal Cancer, 2015;14(3):146-53.

33. Fahmueller YN, Nagel D, Hoffmann RT, Tatsch $\mathrm{K}$ Jakobs T, Stieber P, et al. Predictive and prognostic value of circulating nucleosomes and serum biomarkers in patients with metastasized colorectal cancer undergoing Selective Internal Radiation Therapy. BMC Cancer. 2012;12:5.

34. Boas FE, Bodei L, Sofocleous CT. Radioembolization of Colorectal Liver Metastases: Indications, Technique, and Outcomes. J Nucl Med. 2017;58(Suppl 2):104s-115.

35. Duffy MI. Carcinoembryonic antigen as a marke for colorectal cancer: is it clinically useful? Clin Chem. 2001; $47(4): 624-30$.

36. Weiner AA, Gui B, Newman NB, Nosher JL, Yousseff
F, Lu SE, et al. Predictors of Survival after Yttrium-go Radioembolization for Colorectal Cancer Liver Metastases. Journal of vascular and interventional radiology : JVIR. 2018;29(8):1094-100.

37. Lewandowski RJ, Memon K, Mulcahy MF, Hickey R, Marshall K, Williams M, et al. Twelve-year experience of radioembolization for colorectal hepatic metastases in 214 patients: survival by era and chemotherapy. European journal of nuclear medicine and molecular imaging. 2014;41(10):1861-

38. Kennedy AS, Ball D, Cohen SJ, Cohn M, Coldwell DM, Drooz A, et al. Multicenter evaluation of the safety and efficacy of radioembolization in patients with unresectable colorectal liver metastases selected as candidates for $(90) Y$ resin microspheres. Journal of gastrointestinal oncology. 2015;6(2):134-42.

39. Saxena A, Meteling B, Kapoor J, Golani S, Morris DL, Bester L. Is yttrium-go radioembolization a viable treatment option for unresectable, chemorefractory colorectal cancer liver metastases? A large single-center experience of 302 patients. Annals of surgical oncology. 2015;22(3):794-802

40. Sofocleous CT, Petre EN, Gonen M, Brown KT, Solomon SB, Covey AM, et al. CT-guided radiofrequency ablation as a salvage treatment of colorectal cancer hepatic metastases developing after hepatectomy. Journal of vascular and interventional radiology: JVIR. 2011;22(6):755-61.

41. Wang X, Sofocleous CT, Erinjeri JP, Petre EN, Gonen M, Do KG, et al. Margin size is an independent predictor of local tumor progression after ablation of colon cancer liver metastases. Cardiovasc Intervent Radiol. 2013;36(1):166-75

42. Hoffmann RT, Jakobs TF, Kubisch CH, Stemmler HJ, Trumm C, Tatsch K, et al. Radiofrequency ablation after selective internal radiation therapy with 
Yttriumgo microspheres in metastatic liver diseaseIs it feasible? Eur J Radiol. 2010;74(1):199-205.

43. Lim L, Gibbs P, Yip D, Shapiro JD, Dowling R, Smith $D$, et al. A prospective evaluation of treatment with Selective Internal Radiation Therapy (SIR-spheres) in patients with unresectable liver metastases from colorectal cancer previously treated with 5 -FU based chemotherapy. BMC Cancer. 2005;5:132.

44. Ziv E, Bergen M, Yarmohammadi H, Boas FE, Petre $\mathrm{EN}$, Sofocleous $\mathrm{CT}$, et al. $\mathrm{PI} 3 \mathrm{~K}$ pathway mutations are associated with longer time to local progression after radioembolization of colorectal liver metastases. Oncotarget. 2017;8(14):23529-38.

45. Dorr W. Radiobiology of tissue reactions. Ann ICRP. 2015;44(1 Suppl):58-68. 
${ }^{90}$ Y Resin Microspheres

Radioembolization for

Colon Cancer Liver

Metastases Using Full-

Strength Contrast Material

Kurilova I, Beets-Tan RGH, Ulaner GA, Boas FE, Petre EN, Yarmohammadi H, Ziv E, Deipolyi AR, Brody LA, Gonen M, Sofocleous CT.

Cardiovasc Intervent Radiol. 2018 Sep;41(9):1419-1427. doi: 10.1007/soo270-018-1985-1. Epub 2018 May 15 


\section{ABSTRACT}

Objectives

To assess safety and efficacy of ${ }^{\circ} \mathrm{Y}$ resin microspheres administration using undiluted non-ionic contrast material (UDCM) \{100\% Omnipaque-30o (Iohexol)\} in both the "B" and " $\mathrm{D}$ " lines.

\section{Materials and methods}

We reviewed all colorectal cancer liver metastases patients treated with ${ }^{\circ} Y$ resin microspheres radioembolization (RE) from 2009 to 2017. As of April 2013, two experienced operators started using UDCM (study group) instead of standard sandwich infusion (control group). Occurrence of myelosuppression (leukopenia, neutropenia, erythrocytopenia or/and thrombocytopenia), stasis, non-target delivery (NTD), median fluoroscopy radiation dose (FRD), median infusion time (IT), liver progression-free (LPFS) and overall survivals (OS) was evaluated. Complications within 6 months post-RE were reported according to CTCAE v3.o criteria.

Results

Study and control groups comprised $23(28 \%)$ and $58(72 \%)$ patients, respectively. Median follow-up was 9.1 months. There was no statistically significant difference in myelosuppression incidence within 6 months post-RE between groups. Median FRD and IT for study and control groups were 44.6 vs. $97.35 \mathrm{~Gy} / \mathrm{cm}^{2}(p=0.048)$ and 31 vs. $39 \mathrm{~min}$ $(p=0.006)$, respectively. A $38 \%$ lower stasis incidence in study group was not significant $(p=0.34)$. NTD occurred in $1 / 27(4 \%)$ study vs. $5 / 73(7 \%)$ control group procedures $(p=1)$. Grade 1-2 and grade 3-4 toxicities between study and control group patients were $36 \%$ (8/22) vs. $45 \%$ (26/58), $p=0.61$ and $9 \%(2 / 22)$ vs. $16 \%(9 / 58), p=0.72$, respectively. There was no difference in LPFS and OS between groups.

\section{Conclusion}

Administration of ${ }^{\circ} \mathrm{Y}$ resin microspheres using UDCM in both lines is safe and effective, resulting in lower fluoroscopy radiation dose and shorter infusion time, without evidence of myelosuppression or increased stasis incidence.

\section{INTRODUCTION}

${ }^{90}$ Y resin microspheres (SIR-Spheres-Sirtex Medical, Sydney, Australia) radioembolization (RAE) is an FDA approved brachytherapy device, recommended through the National Comprehensive Cancer Network and European Society for Medical oncology guidelines for the treatment of chemo-refractory colorectal cancer liver metastases (CLM) in selected patients with liver-only or liver-dominant disease [1-13]. The manufacturer recommends using a sandwich technique during ${ }^{90} \mathrm{Y}$ resin microspheres administration $[14,15]$. This technique includes a vehicle $\left\{\right.$ sterile water or $5 \%$ dextrose solution $\left.\left(D_{5} W\right)\right\}$ to preclude direct contact of iodinated contrast material with the resin microspheres. With the sandwich technique, the contrast agent is never used in the "D" line (authorized user line). Since neither the ${ }^{\circ} Y$ microspheres nor the vehicle is radiopaque, the sandwich technique does not allow continuous monitoring of the microsphere flow toward the target tumor. This limits the ability to detect microspheres' stasis or reflux, which can result in nontarget delivery with unnecessary toxicity, as well as diminished radioactivity reaching the target tumor. This is particularly important when treating relatively hypovascular tumors such as CLM, where the incidence of stasis may be higher than $30 \%$ [16-18]

Although the administration of ${ }^{\circ} \mathrm{Y}$ resin microspheres with contrast agent in the "D" line is not recommended [15], there are only anecdotal and controversial data regarding potential disadvantages of this technique. The main concern of using a contrast agent as the delivery medium during ${ }^{90} \mathrm{Y}$ resin microspheres administration includes a theoretical possibility of ${ }^{\circ} \mathrm{Y}$ leaching from the resin microspheres. This could cause systemic non-target radiation delivery [19]. Resin-based yttrium microspheres normally have $0.01-0.4 \%$ unbound or unprecipitated ${ }^{\circ} \mathrm{Y}$ [20], with recommended amount of ${ }^{9} \mathrm{Y}$ leaching of less than $5 \%$ [21]. Another concern is the possibility of microspheres clumping, resulting in microcatheter occlusion. This was observed in vitro in the 1990's, when resin microspheres were exposed to ionic contrast medium, although the details of that experiment remain unknown. Resin microspheres aggregation did not occur with isotonic solutions of non-ionic contrast agent and 5\% dextrose [22]

The safety of a modified ${ }^{9} \mathrm{Y}$ microspheres infusion techniques using diluted nonionic contrast agent in the "B" line-only $[14,22]$ or in both "B" and "D" lines has been demonstrated [23]. The administration of ${ }^{\circ} \mathrm{Y}$ resin microspheres using 50\% nonionic contrast agent in both lines resulted in lower incidence of stasis, shorter resin microspheres infusion and fluoroscopy times without complications [23].

Fluoroscopic image conspicuity is improved as the opacity degree increases. This is directly 
proportional to the concentration of iodinated contrast agent used [24]. Since no adverse event was noted when using diluted contrast material, we started using undiluted contrast medium for ${ }^{\circ} \mathrm{Y}$ resin microspheres administration in 2013. This work was undertaken to specifically address the safety and efficacy of ${ }^{\circ} \mathrm{Y}$ resin microspheres RAE for CLM using undiluted non-ionic contrast material (UDCM) in both "B" and " $\mathrm{D}$ " lines.

\section{MATERIALS AND METHODS}

IRB waiver of approval was obtained for this retrospective review of our prospectively created and maintained HIPAA registered and compliant CLM RAE database. All patients with CLM treated with ${ }^{90} \mathrm{Y}$ resin microspheres RAE from 9/15/2009 to 1/19/2017 were included. Beginning in April 2013, two experienced operators started using 100\% nonionic contrast agent $\{100 \%$ Omnipaque-30o (Iohexol $300 \mathrm{mgl} / \mathrm{ml}$, GE Healthcare Inc.)\} in both "B" and "D" lines (study group) instead of the standard sandwich infusion technique (control group) for ${ }^{\circ} \mathrm{Y}$ resin microspheres administration. In the study group, intermittent clearance with $\mathrm{D}_{5} \mathrm{~W}$ or sterile water solution was performed from both "B" and "D" lines as needed not unlike any other embolization technique. Standard sandwich technique for microspheres administration was used in the control group per manufacturer's instructions, which included administration of sterile water or D5W in both "B" and "D" lines with intermittent checking for the progress of radioembolization by using non-ionic contrast agent in "B" line.

Primary study objectives were as follows:

(1) Comparison of incidence of myelosuppression and lymphopenia within 6 months post-RAE between the groups;

(2) Comparison of incidence of stasis and/or reflux during RAE between the groups;

(3) Comparison of non-target microspheres delivery between the groups;

(4) Comparison of incidence of RAE-related hyperbilirubinemia, side effects and complications within 6 months post-RAE;

(5) Comparison of ${ }^{\circ} \mathrm{Y}$ infusion time between the groups;

(6) Comparison of fluoroscopic radiation dose between the groups;

(7) Comparison of UDCM amount administered between the groups.

Secondary study objectives included:

(1) Analysis of mean laboratory values changes post- RAE between the groups

(2) Liver progression-free survival (LPFS);

(3) Overall survival (OS)
Definitions of study objectives were presented in Supplement 1. All patients were followed at 1-4, 5-8, 9-16 and 17-26 weeks after RAE with clinic visits and laboratory tests to evaluate for possible radiation-induced hepatotoxicity, systemic and any other toxic effect. All laboratory values were recorded by the blinded reader at abovementioned follow-up time points and compared to baseline. In cases of multiple laboratory values within a specific follow-up period available, the value closest to the middle of the specific period was chosen.

Imaging follow-up was obtained at 5-8 weeks and 9-16 weeks post-procedure to assess treatment response and every 3 months thereafter to detect disease progression. Statistical analysis methodology was described in Supplement 2.

\section{RESULTS}

Eighty-one patients underwent $146{ }^{9 \circ} \mathrm{Y}$ resin microspheres infusions. In $23(28 \%)$ patients, ${ }^{9} \mathrm{Y}$ delivery was performed using UDCM (study group), whereas in 58 (72\%) patients standard sandwich infusion technique was used (control group). Median follow-up time was 9.1 months. One patient in study group was lost to follow-up. Study and control group demographics and post-RAE therapies were described in Supplements 3-4.

Complete Blood Count (CBC) Values Change After Radioembolization: Evidence of Myelosuppression and Lymphopenia

Laboratory values on at least one follow-up time point were available for $20 / 23(87 \%)$ patients in the study and 56/58 (97\%) patients in the control group. On the last followup, laboratory values were available for $13 / 23(57 \%)$ patients in the study and $40 / 58$ (69\%) in the control group. Analysis of $\mathrm{CBC}$ values at baseline and follow-ups between the study and control groups showed no evidence to support myelosuppression (Tables 1-2, Figures 1-5). When comparing study and control groups, the incidence of lymphopenia was statistically significantly lower in the study group at $5-8$ weeks post-RAE $(p=0.05)$.

Detailed description of low CBC values within 6 months post-RAE with related clinical symptoms and treatment in the study group patients was described in Supplement 5.

Incidence of Stasis, Catheter Occlusion and Amount of Prescribed Radiation Dose Delivered Incidence of stasis in study and control groups was 23\% (95\% CI 10-36\%) vs. 28\% (95\% CI 19$37 \%$ ). When accounting for multiple infusions per patients using generalized estimated equations test, the study group patients had $38 \%$ lower incidence of stasis (HR o.62, 
$\mathrm{p}=0.34)$. The mean percentage of prescribed ${ }^{\circ} \mathrm{Y}$ dose administered was $90 \%$ in study group ( $95 \%$ CI $85-95 \%$ ) versus $85 \%$ in control group ( $95 \%$ CI 81-89\%), p = 0.21. No catheter occlusion was noted.

Table 1 Incidence of myelosuppression within 6 months after ${ }^{\circ} Y$ radioembolization

\begin{tabular}{|c|c|c|c|}
\hline \multirow{2}{*}{\multicolumn{4}{|c|}{$\begin{array}{l}\text { Laboratory value } \\
\text { Leucopenia }(<4 \mathrm{~K} / \mathrm{mcl})\end{array}$}} \\
\hline & & & \\
\hline Before treatment & $10 / 57(17 \%)$ & $\mathrm{o} / 22(0 \%)$ & 0.06 \\
\hline After 1-4 weeks & $11 / 53(21 \%)$ & 1/15(7\%) & 0.28 \\
\hline After 5-8 weeks & $15 / 54(28 \%)$ & $3 / 17(18 \%)$ & 0.53 \\
\hline After 9-16 weeks & $16 / 49(33 \%)$ & $3 / 14(21 \%)$ & 0.52 \\
\hline After $17-26$ weeks & $10 / 40(25 \%)$ & $3 / 13(23 \%)$ & 1 \\
\hline \multicolumn{4}{|c|}{ Neutropenia $(<1.5 \mathrm{~K} / \mathrm{mcl})$} \\
\hline Before treatment & $2 / 57(4 \%)$ & $\mathrm{o} / 22(0 \%)$ & 1 \\
\hline After 1-4 weeks & $1 / 53(2 \%)$ & $\mathrm{o} / 15(0 \%)$ & 1 \\
\hline After $5-8$ weeks & $2 / 54(4 \%)$ & $0 / 17(0 \%)$ & 1 \\
\hline After 9-16 weeks & $5 / 49(10 \%)$ & $0 / 14(0 \%)$ & 0.17 \\
\hline After $17-26$ weeks & $2 / 40(5 \%)$ & $3 / 13(23 \%)$ & 0.09 \\
\hline \multicolumn{4}{|c|}{ Erythrocytopenia $(<4.2 \mathrm{M} / \mathrm{mcl})$} \\
\hline Before treatment & $31 / 57(54 \%)$ & $15 / 22(68 \%)$ & 0.32 \\
\hline After 1-4 weeks & $30 / 53(57 \%)$ & $11 / 15(73 \%)$ & \\
\hline After $5-8$ weeks & $29 / 54(54 \%)$ & $\begin{array}{l}10 / 17(59 \%) \\
\end{array}$ & 0.78 \\
\hline After $9-16$ weeks & $23 / 49(47 \%)$ & $10 / 14(71 \%)$ & 0.14 \\
\hline After $17-26$ weeks & $24 / 40(60 \%)$ & $8 / 13(62 \%)$ & 1 \\
\hline \multicolumn{4}{|c|}{ Thrombocytopenia $(<160 \mathrm{~K} / \mathrm{mcl})$} \\
\hline Before treatment & $24 / 57(42 \%)$ & $8 / 22(36 \%)$ & 0.08 \\
\hline After 1-4 weeks & $20 / 53(38 \%)$ & $8 / 15(53 \%)$ & 0.37 \\
\hline After 5-8 weeks & $28 / 54(52 \%)$ & $5 / 17(29 \%)$ & 0.16 \\
\hline After 9-16 weeks & $25 / 49(51 \%)$ & $9 / 14(64 \%)$ & 0.54 \\
\hline After $17-26$ weeks & $21 \mid 40(53 \%)$ & $9 / 13(69 \%)$ & 0.35 \\
\hline \multicolumn{4}{|c|}{ Lymphocytopenia $(<0.5 \mathrm{~K} / \mathrm{mcl})$} \\
\hline Before treatment & $3 / 55(5 \%)$ & $3 / 20(15 \%)$ & 0.33 \\
\hline After 1-4 weeks & $15 / 50(30 \%)$ & $4 / 16(25 \%)$ & \\
\hline After $5^{-8}$ weeks & $16 / 50(32 \%)$ & $1 / 16(6 \%)$ & 0.05 \\
\hline After 9-16 weeks & $10 / 47(21 \%)$ & $3 / 13(23 \%)$ & 1 \\
\hline After $17-26$ weeks & $8 / 38(21 \%)$ & $0 / 13(0 \%)$ & 0.17 \\
\hline
\end{tabular}

Table 2 Complete blood count value changes after RAE

\begin{tabular}{llll}
\hline Laboratory value & Coefficient $^{\mathrm{a}}$ & $\boldsymbol{P v a l u e}$ & $\mathbf{9 5 \%} \mathrm{CI}$ \\
\hline WBC $(\mathrm{K} / \mathrm{mcl})$ Diff between infusion groups & 0.02 & 0.98 & {$[-1,59 ; 1,63]$} \\
ANC $(\mathrm{K} / \mathrm{mcl})$ Diff between infusion groups & -0.4 & 0.95 & {$[-1,46 ; 1,37]$} \\
RBC $(\mathrm{M} / \mathrm{mcl})$ Diff between infusion groups & 0.11 & 0.17 & {$[-1,46 ; 1,37]$} \\
PLT $(\mathrm{K} / \mathrm{mcl})$ Diff between infusion groups & -10.21 & 0.45 & {$[-36.41 ; 16.0]$} \\
ALC $(\mathrm{K} / \mathrm{mcl})$ Diff between infusion groups & 0.14 & 0.11 & {$[-0.03 ; 30]$} \\
\hline Analysis was made using multilevel mixed-effects linear regression model & &
\end{tabular}

Analysis was made using multilevel mixed-effects linear regression model

Diff-difference; WBC-white blood cell count, ANC-absolute neutrophil count, RBC-red blood cell count, PLTplatelet count, ALC-absolute lymphocyte count. There was no statistically significant difference between study and control groups complete blood count hanges over 6 months period. "Negative coefficients reflect lower mean values in study group during follow-up period, positive coefficients-higher mean values in study grou

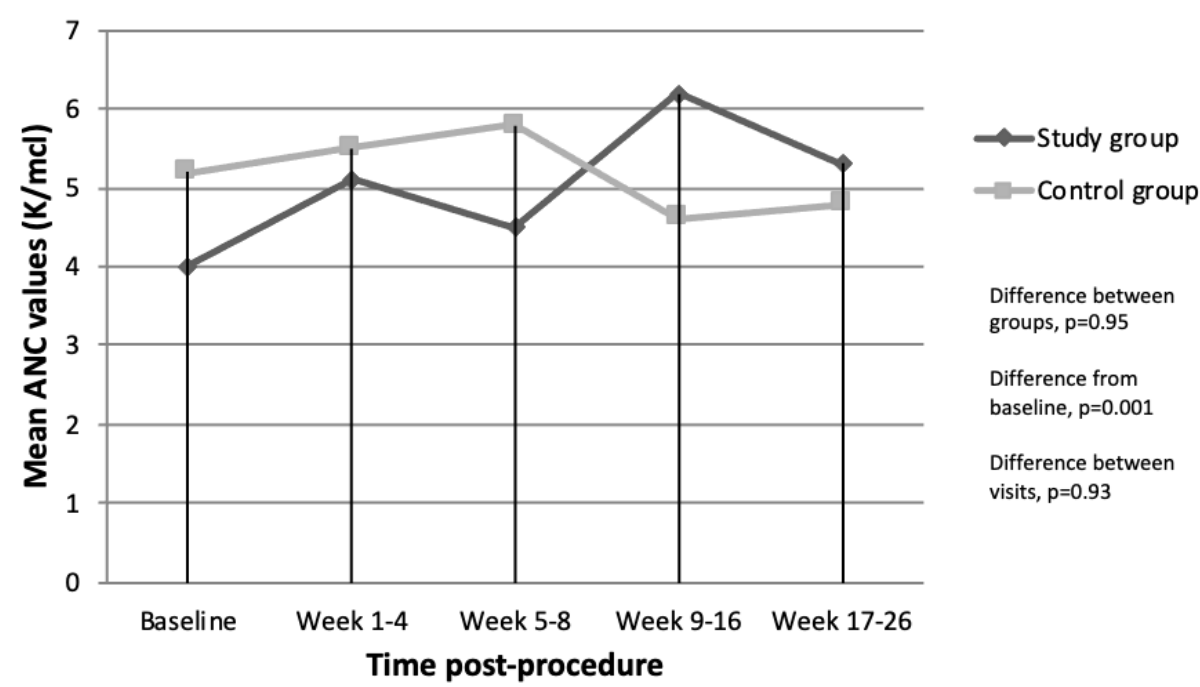

Figure 1 Mean white blood cell count (WBC) values within 6 months post-radiomebolization (accounted for multiple treatment sessions per patient). The figure demonstrates that there was no statistically significant difference in mean white blood cell count between study and control groups $(\mathrm{p}=0.98)$

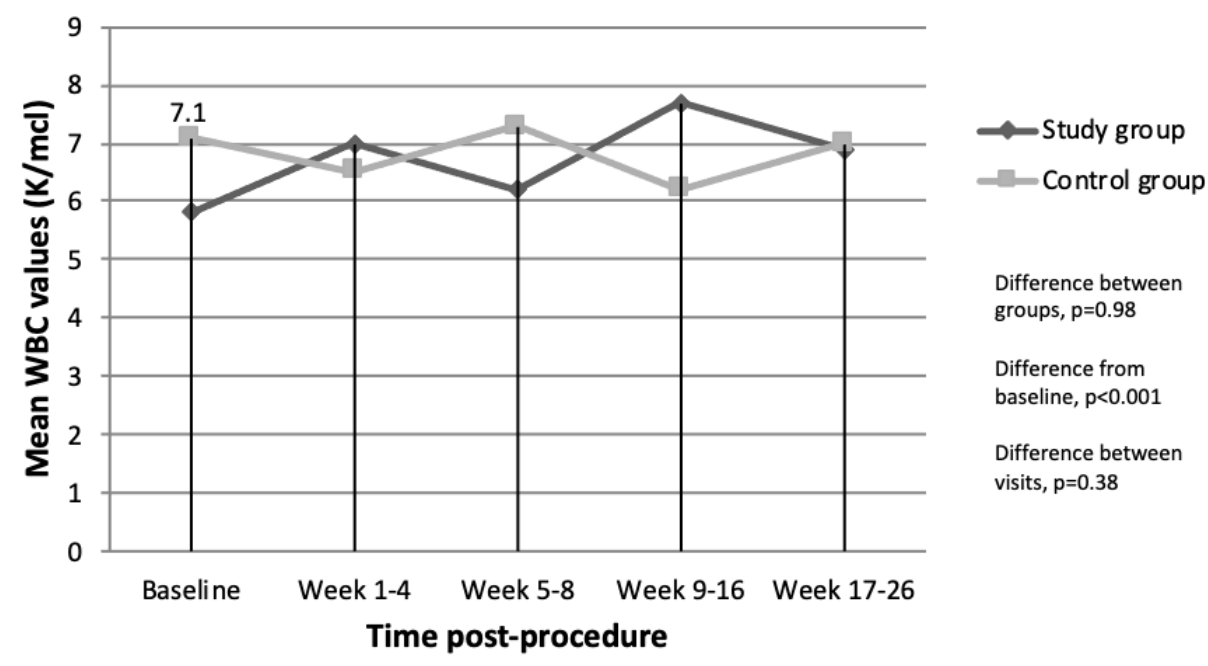

Figure 2 Mean absolute neutrophil count (ANC) values within 6 months post-radiomebolization. The figure demonstrates that there was no statistically significant difference between study and control groups $(\mathrm{p}=0.95)$ 


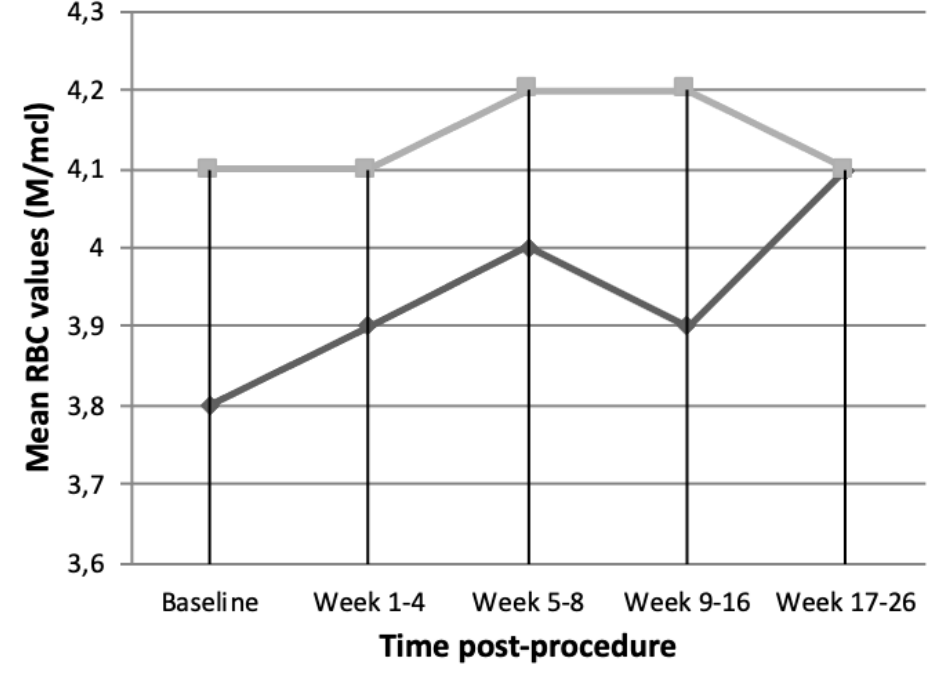

\section{$\sim$ Study group}

$\rightarrow-$ Control group

Difference between

groups, $\mathrm{p}=0.17$

Difference from
baseline, $p<0.001$

Difference between

Difference betwe
visits, $\mathrm{p}=0.08$ demonstrates that there was no statistically significant difference between study and control groups ( $\mathrm{P}$ $=0.17)$

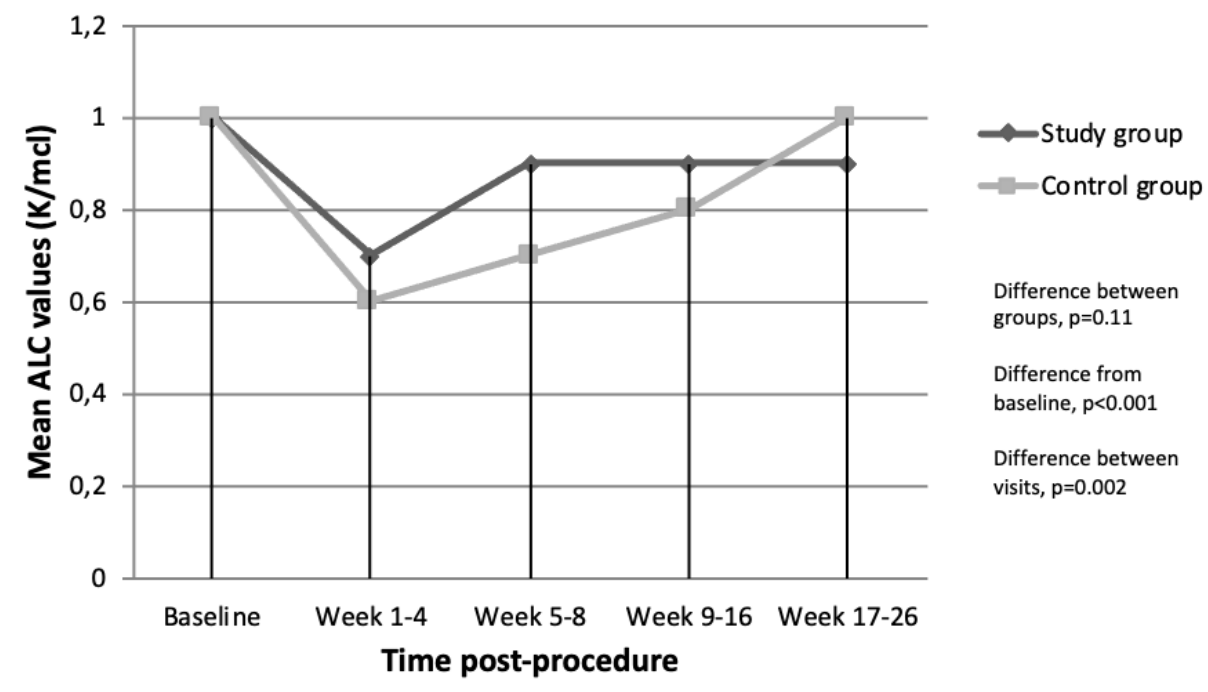

Figure 4 Mean platelet count (PLT) values within 6 months post-radiomebolization. The figure

demonstrates that there was no statistically significant difference between study and control groups ( $\mathrm{p}$ $=0.45)$

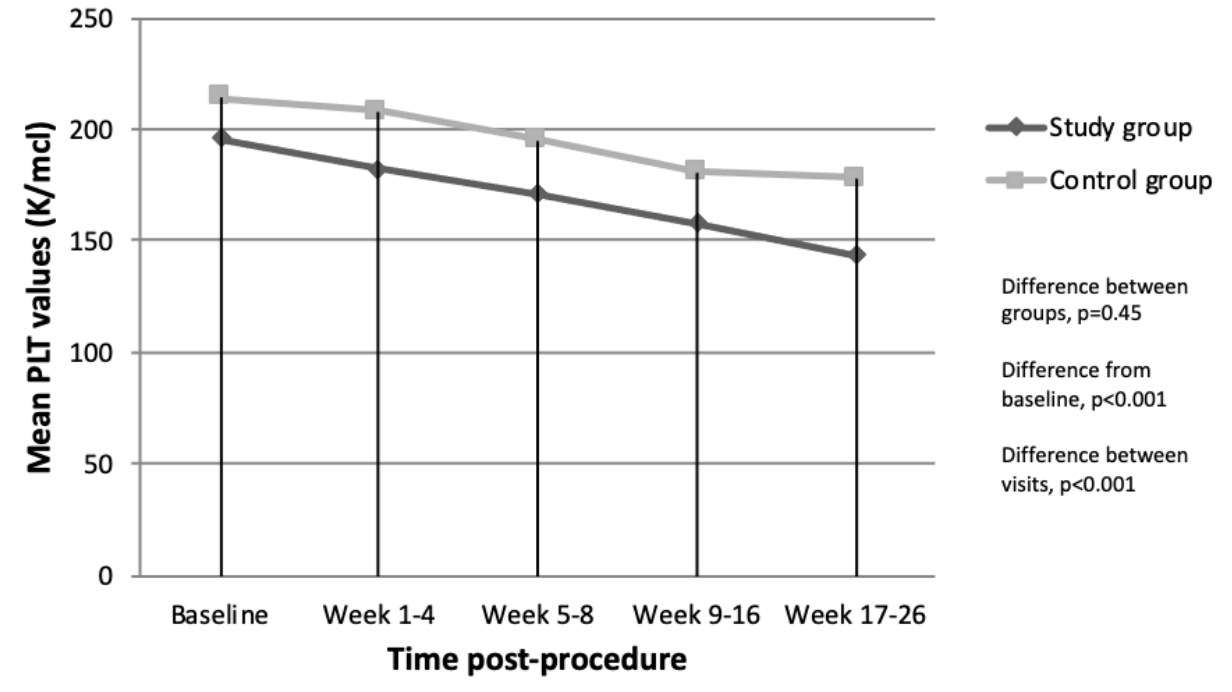

Figure 5 Mean absolute lymphocyte count (ALC) values within 6 months post-radiomebolization. The figure demonstrates that there was no statistically significant difference between study and control $\operatorname{groups}(\mathrm{p}=0.11)$

Median Fluoroscopy Radiation Dose, Microspheres Infusion Time and Amount of Used UDCM Median microsphere infusion time was 31 vs. 39 min for the study vs. control groups ( $\mathrm{p}=$ o.oo6). A total of 71 single-infusion procedures were identified and used for the median fluoroscopy radiation dose and median amount of administered UDCM analyses; 8/71 (11\%) infusions were excluded from the analysis as they required additional vascular embolization prior to RAE. After exclusion, 12 procedures in the study and 51 in the control group were analyzed.

Median single-infusion RAE fluoroscopy radiation dose for the study and control groups was $44.6 \mathrm{~Gy} / \mathrm{cm} 2$ (range, 19.4-221.8 Gy/cm2) and 97.35 Gy/cm2 (range, 23.2-398.2 Gy/cm2), respectively, $\mathrm{p}=0.048$. There was no statistically significant difference in mean patients BMI values between study (26.6) and control group (28.4), p = 0.3. Median amount of UDCM administered in the study and control groups was exactly the same: $150 \mathrm{ml}$ (range, 101-200 ml) and $150 \mathrm{ml}$ (range, 60-275 ml), respectively, $\mathrm{p}=0.72$ (Table 3). 
Table 3 Comparison of time from resin microspheres infusion start to post-implantation vial radioactivity measurement, fluoroscopy radiation doses and amount on contrast medium (Omnipaque-30o) administered between study and control groups

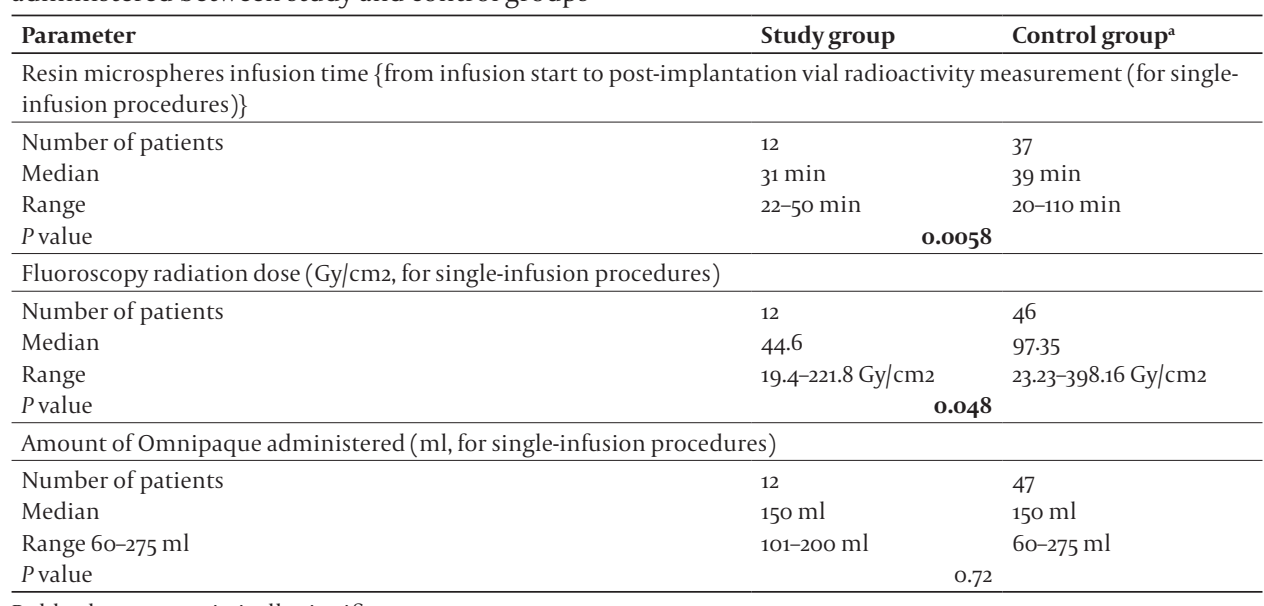

Bold values are statistically significant

'Analyzed parameters data were not available per reports for all control group patients. The table demonstrated in study group $(p=0.048)$, compared to control group. There was no statistically significant diffescopy radiation dose regarding amount of contrast medium (Omnipaque) administered.

Incidence of Reflux and Evidence of Non-target Delivery on Bremsstrahlung SPECT/CT Imaging Reflux was not observed in the study group. It was recorded in 3/58 (5\%) of infusions in the control group. The incidence of non-target ${ }^{\circ} \mathrm{Y}$ delivery on Bremsstrahlung SPECT/CT between study and control groups was $1 / 27$ (4\%, 95\% CI o-11\%) vs. 5/73 (7\%, 95\% CI 1-13\%), p $=1$. In the study group, tracer uptake along the distribution of the falciform artery was recorded. The vessel was identified in pretreatment mapping, but could not be embolized. Ice packs were placed on the skin during ${ }^{90} Y$ microspheres delivery; however, activity in the distribution of this vessel was detected in the Bremsstrahlung SPECT/CT scan [25]. The patient remained asymptomatic and without skin changes during follow-up.

Side Effects and Complications after ${ }^{\circ} Y$ Radioembolization

Side effects during ${ }^{90} \mathrm{Y}$ microspheres infusion were observed only in the control group (in 6/58 (9\%) of patients), which included pain, hypersensitivity reaction (flushing and swelling) and arterial hypertension. There were no side effects in the study group. There was no difference in incidence of hyperbilirubinemia, grade $1-2$ and grade 3-4 toxicities (Tables 4, 5).
Table 4 Grade 1-2 toxicities

\begin{tabular}{lll}
\hline Complication $^{*}$ & Study group & Control group \\
\hline Fatigue/weakness & $5 / 22(23 \%)$ & $26 / 58(45 \%)$ \\
Abdominal pain/distension & $3 / 22(14 \%)$ & $1 / 158(29 \%)$ \\
Shortness of breath & $1 / 22(5 \%)$ & $9 / 58(16 \%)$ \\
Fever & $1 / 22(5 \%)$ & $9 / 58(16 \%)$ \\
Change in appetite/weight changes & 0 & $8 / 58(14 \%)$ \\
Nausea & $1 / 22(5 \%)$ & $7 / 58(12 \%)$ \\
Vomiting & 0 & $4 / 58(7 \%)$ \\
Ascites & 0 & $5 / 58(9 \%)$ \\
Constipation/diarrhoea & 0 & $13 / 58(45 \%)$ \\
Light coloured stool/dark urine & 0 & $9 / 58(16 \%)$ \\
Hiccups & 0 & $1 / 58(2 \%)$ \\
Portal hypertension & $1 / 22(5 \%)$ & 0 \\
Total patients number & $\mathbf{8 / 2 2}(36 \%)$ & $\mathbf{2 6 / 5 8 ( 4 5 \% )}$ \\
\hline
\end{tabular}

"Some of the patient might have developed multiple complications

Table 5 Grade 3-4 toxicities

\begin{tabular}{llll} 
Complication & Days post-RE & Study group & Control group \\
\hline Post-RE radioembolization syndrome & $1-90$ days & $1 / 22(5 \%)$ & $4 / 58(7 \%)$ \\
Grade 3 abdominal pain/distension & $10-12$ days & $1 / 22(5 \%)$ & $2 / 58(3 \%)$ \\
Grade 3 fevers & 3 days & 0 & $1 / 58(2 \%)$ \\
Acute liver failure/failure to thrive/grade 3 hyperbilirubinemia & 14 days & 0 & $1 / 58(2 \%)$ \\
Grade 3 dyspnoea & 30 days & 0 & $1 / 58(2 \%)$ \\
$\begin{array}{l}\text { Bile duct obstruction + Budd-Chiari syndrome + stop } \\
\text { chemotherapy for 6 weeks }\end{array}$ & 1 day/60 days & $1 / 22(5 \%)$ & 0 \\
Total patients number & & $2 / 22(9 \%)$ & $\mathbf{9 / 5 8 ( 1 6 \% )}$ \\
\hline
\end{tabular}

*Some of the patient might have developed multiple complications. RE-radioembolization

Median Liver PFS and OS

Median liver PFS for study and control groups was 4.13 vs. 3.97 months $(H R=1.29, \mathrm{p}=$ o.38) (Fig. 6). OS for study and control groups was 11.6 months vs. 9 months ( $p=0.74$, HR = 0.9) $($ Fig. 7) 


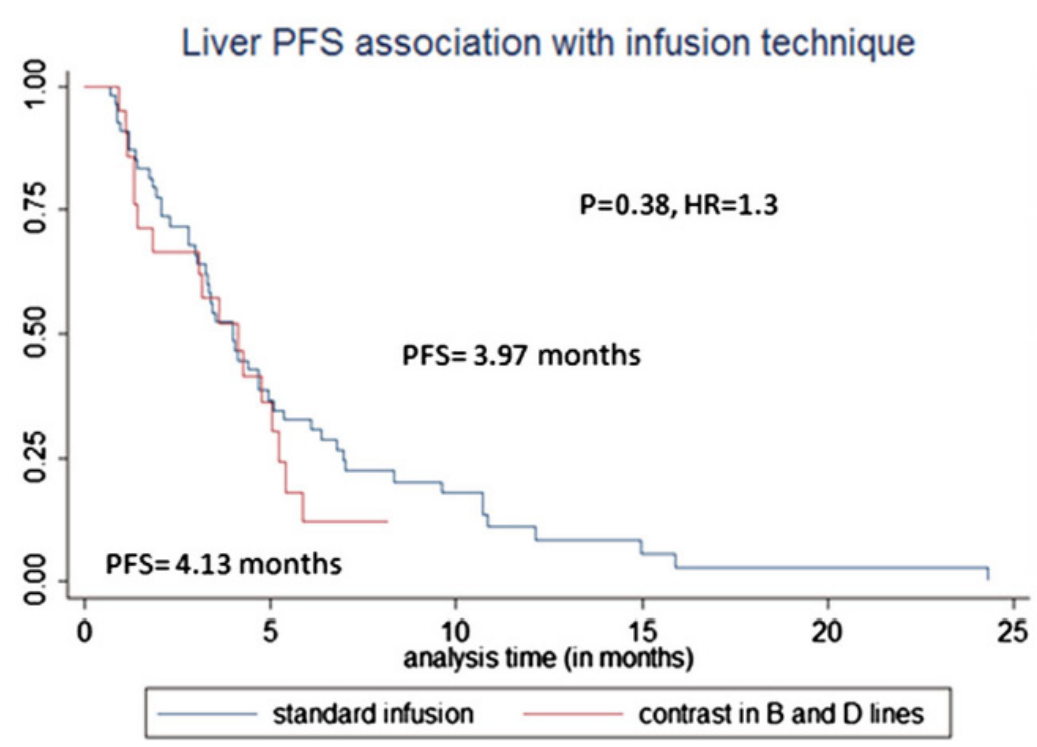

Figure 6 Liver progression-free survival (PFS) association by infusion technique. The figure demonstrated that there was no statistically significant difference in liver PFS between study and control groups $(\mathrm{p}=0.38)$

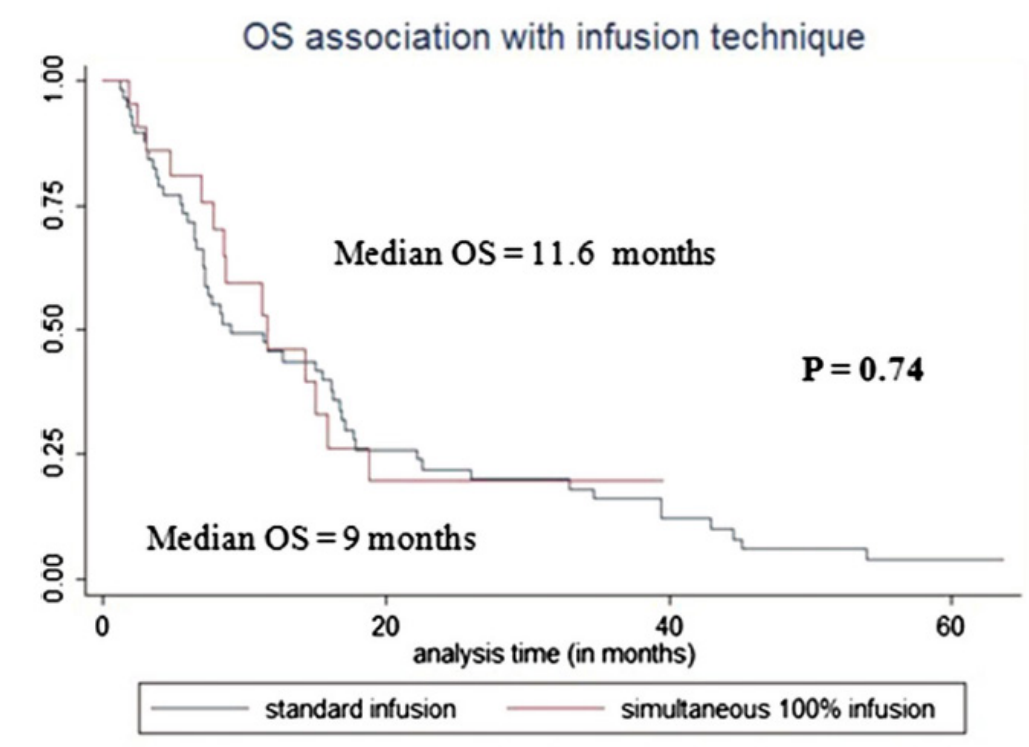

Figure 7 Overall survival (OS) association with infusion technique. The figure demonstrated that there was no statistically significant difference in OS between study group (simultaneous 100\% contrast medium infusion) and control group (standard sandwich technique infusion), $\mathrm{p}=0.74$

\section{DISCUSSION}

This study demonstrated no indirect evidence of $9^{\circ} \mathrm{Y}$ leaching when using UDCM for ${ }^{9 \circ} \mathrm{Y}$ resin microspheres administration in both "B" and " $\mathrm{D}$ " lines. This was indicated by no difference in complete blood count values at baseline and follow-ups between the study and control groups. The comparison with the control group was used as reference to anticipated complete blood count changes post-RAE, such as thrombocytopenia and lymphopenia [26-28]. No abnormal $\beta$-emission was detected on Bremsstrahlung SPECT/ $\mathrm{CT}$ in the study group, which further supported the lack of ${ }^{\circ} \mathrm{Y}$ leaching in the study. There was no difference in peri- or post-procedural toxicity, hepatic progression-free and overall survival between the groups. These findings indicated that no additive toxicity occurred during direct infusion of resin microspheres using UDCM. Chao et al., similarly, reported no complications when infusing ${ }^{\circ} Y$ resin microspheres with $50 \%$ non-ionic contrast medium in saline [23].

Statistically significantly lower median fluoroscopy radiation dose was recorded in the study group, compared to control ( 44.6 vs. $97.4 \mathrm{~Gy} / \mathrm{cm} 2, \mathrm{p}=0.048$ ), accounting for patients' BMI. The amount of contrast agent used was similar between the groups. A significantly shorter median resin microspheres infusion time in the study group may have attributed to the lower fluoroscopy radiation dose and the same amount of contrast agent used between groups. This is concordant with reported findings by Chao et al. (7 min infusion time for the $50 \%$ of Isovue vs. 22 min for the standard sandwich technique) [23]. A38\% reduction in the incidence of stasis in the study group did not reach statistical significance in our study. However, when using $50 \%$ of concentration non-ionic contrast medium, a significant decrease in the incidence of stasis was documented [23]. Reflux never occurred during UDCM infusion in our study, compared to three cases in the control group. Non-target delivery occurred in 5/73 (7\%) of infusions in the control group compared to $1 / 27(4 \%)$ in the study group (all asymptomatic).

No gastrointestinal ulceration was noted, comparing favorably to historical data. Gastrointestinal ulceration is the most frequent major complication, reported in $0-20 \%$ of RAEs with a median incidence of $8 \%$ when using advanced antireflux protective techniques and in $6 \%$ of cases requiring surgical ulcer management [29]. ${ }^{\circ} \mathrm{Y}$ administration using UDCM was as effective as sandwich technique in terms of liver progression-free and overall survival.

Limitations of the study include its single-center, retrospective nature and the relatively small number of patients. The lack of pathologic assessment such as bone marrow biopsy 
at different time points after RAE to detect early microscopic bone marrow morphologic changes was another study limitation. Direct ${ }^{\circ} Y$ leaching detection should be performed in blood or urine samples in certified radioactive material laboratories, according to radiation safety standards [21]. The lack of these assessments was limitations of this study. Leaching and radiotoxicity were assessed only indirectly, through post-RAE serial complete blood count, Bremsstrahlung SPECT/CT as well as laboratory and clinical toxicity evaluations. The fact, that ${ }^{\circ} \mathrm{Y}$ Bremsstrahlung SPECT/CT has low quantitative accuracy and that it was only done at one-time point (within hours of RAE, according to routine practice), limits the potential for leaching detection [30, 31]. Considering that the half-life of ${ }^{9} \mathrm{Y}$ is 2.7 days, ideally such study should be performed in several time points within 11 days after RAE to detect all emitted $\beta$-radiation in both target and nontarget organs. However, such information would have little if any impact on clinical patient management due to the absence of symptoms and laboratory values, indicating myelosuppression and radiotoxicity. Further prospective studies could address these limitations and optimize the direct administration of ${ }^{\circ} \mathrm{Y}$ resin microspheres with nonionic contrast medium.

\section{CONCLUSION}

Despite the limitations, the study met its goal and demonstrated that direct ${ }^{\circ} Y$ resin microspheres infusion with undiluted non-ionic contrast medium was safe and effective. Significant reduction in ${ }^{9} \mathrm{Y}$ resin microspheres infusion time and fluoroscopic radiation exposure were documented.

\section{REFERENCES}

1. Benson AB 3rd, Venook AP, Cederquist L, Chan E, Chen YJ, Cooper HS, et al. Colon Cancer, Version 1.2017, NCCN Clinical Practice Guidelines in Oncology. J Nat Comprehen Cancer Netw JNCCN. 2017:15(3):370-98.

2. Van Cutsem E, Cervantes A, Adam R, Sobrero A, Van Krieken JH, Aderka D, et al. ESMO consensus guidelines for the management of patients with metastatic colorectal cancer. Ann Oncol Off J Eur Soc Med Oncol. 2016;27(8):1386-422.

3. Kennedy A, Nag S, Salem R, Murthy R, McEwan $\mathrm{A}$, Nutting $\mathrm{C}$, et al. Recommendations for radioembolization of hepatic malignancies using yttrium-9o microsphere brachytherapy: a consensus panel report from the radioembolization brachytherapy oncology consortium. Int J Radiat Oncol Biol Phys. 2007;68(1):13-23.

4. Sotirchos VS, Petre EN, Brown KT, Brody LA, D’Angelica MI, DeMatteo RP, et al. Safe and successful yttrium-go resin microsphere radioembolization in a heavily pretreated patient with chemorefractory colorectal liver metastases after biliary stent placement above the Papilla. Case Rep Hepatol. 2014;2014:921406.

5. Sofocleous CT, Garcia AR, Pandit-Taskar N, Do KG, Brody LA, Petre EN, et al. Phase I trial of selective internal radiation therapy for chemorefractory colorectal cancer liver metastases progressing after hepatic arterial pump and systemic chemotherapy. Clin Colorectal Cancer. 2014;13(1):27-36.

6. Hendlisz A, Van den Eynde M, Peeters M, Maleux G, Lambert B, Vannoote J, et al. Phase III trial comparing protracted intravenous fluorouracil infusion alone or with yttrium-9o resin microspheres radioembolization for liver-limited metastatic colorectal cancer refractory to standard chemotherapy. JClin Oncol Off] J Am Soc Clin Oncol. 2010;28(23):3687-94

Van Hazel G, Blackwell A, Anderson J, Price D, Moroz P, Bower G, et al. Randomised phase 2 trial of SIR-Spheres plus fluorouracil/ leucovorin chemotherapy versus fluorouracil/leucovorin chemotherapy alone in advanced colorectal cancer. J Surg Oncol. 2004;88(2):78-85.

8. Gray B, Van Hazel G, Hope M, Burton M, Moroz P, Anderson J, et al. Randomised trial of SIR-Spheres plus chemotherapy vs. chemotherapy alone for treating patients with liver metastases from primary large bowel cancer. Ann Oncol Off J Eur Soc Med Oncol. 2001:12(12):1711-20.

9. Sharma RA, Wasan HS, Hazel GAV, Heinemann V, Sharma NK, Taieb J, et al. Overall survival analysis of the FOXFIRE prospective randomized studies of first-line selective internal radiotherapy (SIRT) in patients with liver metastases from colorectal cancer. J Clin Oncol 2017:35(15 suppl):3507

10. Kennedy AS, Nutting C, Coldwell D, Gaiser I, Drachenberg C. Pathologic response and microdosimetry of $(90) Y$ microspheres in man: review of four explanted whole livers. Int J Radiat Oncol Biol Phys. 2004;60(5):1552-63.

11. Sharma RA, Van Hazel GA, Morgan B, Berry DP, Blanshard K, Price D, et al. Radioembolization of liver metastases from colorectal cancer using yttrium-go microspheres with concomitant systemic oxaliplatin, fluorouracil, and leucovorin chemotherapy. I Clin Oncol Off J Am Soc Clin Oncol. 2007;25(9):1099-106.

12. Vente MA, Wondergem M, van der Tweel I, van den Bosch MA, Zonnenberg BA, Lam MG, et al. Yttrium-go microsphere radioembolization for the treatment of liver malignancies: a structured meta- 
analysis. Eur Radiol. 2009;19(4):951-9.

13. Cosimelli M, Golfieri R, Cagol PP, Carpanese L, Sciuto R, Maini CL, et al. Multi-centre phase II clinica trial of yttrium-go resin microspheres alone in unresectable, chemotherapy refractory colorectal liver metastases. Br J Cancer. 2010;103(3):324-31

14. Paprottka KJ, Todica A, Ilhan H, Rubenthaler J, Schoeppe F, Michl M, et al. Evaluation of Visualization using a 50/50 (contrast media/glucose $5 \%$ solution) technique for radioembolization as an alternative to a standard sandwich technique. Cardiovasc Interv Radiol. 2017;40(11):1740-7.

15. SIR-Spheres_ Y-9o resin microspheres Package Insert

16. Sofocleous CT, Violari EG, Sotirchos VS, Shady W, Gonen M, Pandit-Taskar N, et al. Radioembolization as a salvage therapy for heavily pretreated patients with colorectal cancer liver metastases: factors that affect outcomes. Clin Colorectal Cancer 2015;14(4):296-305.

17. Piana PM, Bar V, Doyle L, Anne R, Sato T, Eschelman DJ, et al. Early arterial stasis during resin-based yttrium-9o radioembolization: incidence and preliminary outcomes. HPB Off J Int Hepato Pancreato Biliary Assoc. 2014;16(4):336-41.

18. Murthy R, Xiong H, Nunez R, Cohen AC, Barron B, Szklaruk J, et al. Yttrium go resin microspheres for the treatment of unresectable colorectal hepatic metastases after failure of multiple chemotherapy regimens: preliminary results. J Vascul Interv Radiol JVIR. 2005;16(7):937-45.

19. Mantravadi RV, Spigos DG, Tan WS, Felix EL Intraarterial yttrium go in the treatment of hepatic malignancy. Radiology. 1982;142(3):783-6.

20. Lambert B, Sturm E, Mertens J, Oltenfreiter R, Smeets P, Troisi R, et al. Intra-arterial treatment with (9)(o)Y microspheres for hepatocellular carcinoma: 4 year experience at the Ghent
University Hospital. Eur I Nucl Med Mol Imaging. 2011;38(12):2117-24

21. Patents.google.com. (2017). WO2002034300A1Polymer based radionuclide containing particulate material-Google Patents. https:||patents.google. com/patent/WO2002034300A1/en?assignee= Sirtex?Medical?Ltd [Accessed 26 Jul. 2017].

22. Sirtex Medical Ltd. Technical Bulletin, August 7, 2013. Available from Sirtex Medical

23. Chao C, Stavropoulos SW, Mondschein JI, Dagli M, Sudheendra D, Nadolski G, et al. Effect of Substituting $50 \%$ isovue for sterile water as the delivery medium for SIR-Spheres: improved dose delivery and decreased incidence of stasis. Clin Nucl Med. 2017;42(3):176-9.

24. https://www.drugbank.ca/drugs/DBo1362 [3.26.2018].

25. Wang DS, Louie JD, Kothary N, Shah RP, Sze DY. Prophylactic topically applied ice to prevent cutaneous complications of nontarget chemoembolization and radioembolization. I Vascul Interv Radiol JVIR. 2013;24(4):596-600.

26. Formenti SC, Demaria S. Systemic effects of local radiotherapy. Lancet Oncol. 2009;10(7):718-26.

27. Nakamura N, Kusunoki Y, Akiyama M Radiosensitivity of $\mathrm{CD} 4$ or $\mathrm{CD} 8$ positive human T-lymphocytes by an in vitro colony formation assay. Radiat Res. 1990;123(2):224-7.

28. Riaz A, Awais R, Salem R. Side effects of yttrium-9o radioembolization. Front Oncol. 2014;4:198.

29. Murthy R, Brown DB, Salem R, Meranze SG, Coldwell DM, Krishnan S, et al. Gastrointestinal complications associated with hepatic arterial Yttrium-go microsphere therapy. Journal of vascular and interventional radiology: JVIR. 2007; ;8(4):553-61; quiz 62.

30. Dezarn WA, Cessna JT, DeWerd LA, Feng W, Gates VL, Halama J, et al. Recommendations of the American
Association of Physicists in Medicine on dosimetry, imaging, and quality assurance procedures for go microsphere brachytherapy in the treatment of hepatic malignancies. Med Phys. 2011;38(8):4824-45

31. Rong X, Du Y, Ljungberg M, Rault E, Vandenberghe S, Frey EC. Development and evaluation of an improved quantitative $(90) Y$ bremsstrahlung SPECT method. Med Phys. 2012;39(5):2346-58. 


\section{SUPPLEMENTARY MATERIAL}

Supplement 1 Definitions of primary and secondary study objectives

\section{Primary study objectives and definitions}

Incidence of myelosuppression and Myelosuppression was defined as occurrence of leukopenia, neutropenia, lymphopenia $\quad$ erythrocytopenia and/or thrombocytopenia within 6 months post-RAE sensivity to

Non-target microspheres delivery Non-target delivery was determined as extrahepatic or non-target intrahepatic

Incidence of RAE-related hyperbilirubinemia RAE-related hyperbilirubinemia was defined as elevation of total plasma resolved within 1 month, with no evidence of liver disease progression, biliary dilatation or another hepatic pathology

Side effects and complications

Side effects and complic Complications severity was recorded according to CTCAE v3.o criteria within 6

infusion time

It was calculated after review of the timed ${ }^{\circ \mathrm{Y}}$ vial radioactivity measurement

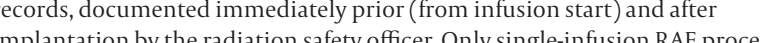
were included for the assessment of this factor RAEs requiring coil-embolization were excluded from analysis

Fluoroscopy radiation dose

Fluoroscopy radiation dose was analysed taking into consideration patient's BML Only single-infusion RAE procedures were included for the assessment of this factor. RAEs requiring coil-embolization were excluded from analysis

$\begin{array}{ll}\text { Amount of UDCM administered } & \begin{array}{l}\text { Amount of UDCM administered during all RAE procedure was recorded in ml. } \\ \text { Only single dose RAE procedures were included for the assessment of this facto }\end{array} \\ \text { Rasions }\end{array}$ RAEs requiring coil-embolization were excluded from analysis

\section{Secondary study objectives and definitions}

Mean laboratory values comparison For patients who underwent two RAE sessions, blood count values before and between groups

Liver progression-free surviva

after each RAE session were recorded

LPFS was defined as the time from initial RAE to documented liver progression (in the treated region or outside treated region after RAE) or until the last imaging follow-up using EORTC and/or RECIST 1.1 criteria, depending on imaging availability. In cases of differing responses between the criteria, the concordance between both modalities imaging findings was used for defining LPFS OS was defined as the time from initial RAE to patient death or last follow-I p
Supplement 2 Overview of statistical analysis methods used in the study

Kaplan Meier method Method was used to estimate patients' overall and liver progression-free survivals

Cox regression analysis Analysis was used to assess patients' overall and liver progression-free survival (LPFS)

Multilevel mixed effects Model was used to evaluate whether there was a statistically significant difference in near regression mode mean blood count values between treatment groups during follow- up period for evaluation of repas chosen as it contains both fixed effects and random effects multiple radiomblization (RAE) sessions per patient and superiority of handling missing values over ANOVA statistical test

Generalized estimated Test was used to evaluate difference in occurrence of stasis and radiation dose delivery equation test dependence between variables (presence of multiple variables at the same or different time points per patient, due to multiple infusions during one RAE session or due to several RAE sessions)

Fisher exact test

Mann-Whitney test Test was used to analyse the difference between two groups regarding not normally

Unpaired t-test distributed continuous variables

Other

Test was used to analyse normally distributed variables

Median follow-up time was calculated for the patients who were alive on last follow- by up. Normal distribution of variables was assessed using Shapiro-Wilk test as well as by evaluating the histograms of the variables, comparing means and medians, skewness and kurtosis of the histogram. Statistical analysis was performed using STATA 12.1 software. A p-value $<0.05$ was considered significant

Supplement 3 Comparison of study and control groups

\begin{tabular}{|c|c|c|c|}
\hline Parameter & Study group & Control group & P-value \\
\hline Number of patients & $23(28 \%)$ & $58(72 \%)$ & - \\
\hline Number of procedures & 27 & 73 & \\
\hline Number of infusions & 39 & 107 & - \\
\hline Mean age (years) & $64 \pm 12.8$ & $56.6 \pm 13$ & 0.047 \\
\hline Gender & & & - \\
\hline Male & $14 / 23(61 \%)$ & $41 / 58(71 \%)$ & \\
\hline Female & $9 / 23(39 \%)$ & $17 / 58(29 \%)$ & \\
\hline $\mathrm{SUV}_{\max }$ before procedure (median, range) ${ }^{*}$ & $14(6.8-30.5)$ & $9 \cdot 3(3.3-28.6)$ & 0.04 \\
\hline \multicolumn{4}{|l|}{ Tumor involvement } \\
\hline Unilobar $^{3}$ & $1 / 23(4 \%)$ & $13 / 58(22 \%)$ & 0.11 \\
\hline Unilobar post hepatectomy & $3 / 23(13 \%)$ & $7 / 58(12 \%)$ & 1 \\
\hline Bilobar & $19 / 23(83 \%)$ & $38 / 58(66 \%)$ & 0.18 \\
\hline Prescribed radiation dose $(\mathrm{mCi}$, mean $\pm \mathrm{SD})$ & $36.2 \pm 12.9$ & $41.1 \pm 14.6$ & \\
\hline Delivered radiation dose $(\mathrm{mCi}$, mean $\pm \mathrm{SD}$ ) & $30.4 \pm 12.8$ & $36 \pm 15.2$ & \\
\hline CEA level before procedure (median, range) & $16.3(5.5-11723)$ & $79 \cdot 5(1.1-23938)$ & 0.016 \\
\hline Synchronous liver metastases on initial diagnosis & $20 / 23(87 \%)$ & $41 / 58(71 \%)$ & 0.25 \\
\hline Lymph nodes involvement on initial diagnosis & $9 / 13(69 \%)$ & $40 / 57(71 \%)$ & 1 \\
\hline Lymphovascular invasion of primary tumor & $8 / 11(73 \%)$ & $27 / 49(55 \%)$ & 0.33 \\
\hline \multicolumn{4}{|l|}{ Tumor differentiation level } \\
\hline Good to moderate & $16 / 21(76 \%)$ & $46 / 57(81 \%)$ & \\
\hline Moderate to poor & $5 / 21(24 \%)$ & $111 / 57(19 \%)$ & 0.75 \\
\hline Prior liver surgery & $14 / 23(61 \%)$ & $50 / 57(88 \%)$ & 0.01 \\
\hline Prior hepatic artery infusion chemotherapy & $13 / 23(57 \%)$ & $30 / 57(53 \%)$ & 0.81 \\
\hline
\end{tabular}

SUV-standard uptake value; mean SUV $_{\max }$ of $1-5$ most metabolically active liver tumours was measured; CEAThe control group patients, on the other hand, had statistically significantly higher baseline CEA level and higher incidence of prior liver surgery for metastatic disease 
Supplement 4 Incidence of hepatic and systemic chemotherapy regimens received post-RAE between the groups

\begin{tabular}{llll}
\hline & Study group & Control group $^{*}$ & P-value \\
\hline Post-RAE hepatic artery infusion chemotherapy & $1 / 23(4 \%)$ & $15 / 58(26 \%)$ & $\mathbf{0 . 0 3}$ \\
No post-RAE systemic chemotherapy & $5 / 23(22 \%)$ & $17 / 57(30 \%)$ & 0.58 \\
1 post-RAE systemic chemotherapy regimen & $12 / 23(52 \%)$ & $17 / 57(30 \%)$ & 0.07 \\
2 post-RAE systemic chemotherapy regimens & $4 / 23(17 \%)$ & $9 / 57(16 \%)$ & 1 \\
Z3 post-RAE systemic chemotherapy regimens & $2 / 23(9 \%)$ & $10 / 57(18 \%)$ & 0.49 \\
\hline "The data of one patient about systemic therapy was not available. RAE-radioembolization. The table demonstrated \\
that control group patients had higher incidence of post-RAE hepatic artery infusion chemotherapy, compared to the \\
study yroup. There was no statistically significant difference in systemic chemotherapy regimens administered post- \\
RAE between the groups
\end{tabular}

RAE between the groups

Supplement 5 Occurrence of low blood count values at any recorded time point within 6 months postRAE with related clinical symptoms and treatment in study group patients

Parameter $(\mathbf{n}=2)$

Erythrocytopenia $(<4.2 \mathrm{M} / \mathrm{mcl})$

Incidence $17(81 \%)$ $\begin{array}{lr}\text { Fatigue, weakness, dizziness, reduced performance status } & 15(71 \%) \\ \text { Shortness of breath } & 6(29 \%)\end{array}$ Required treatment with iron sucrose 1oo $\mathrm{mg}$ IVPB or ferrous gluconate $324 \mathrm{mg} p / 0 \mathrm{~s}$ los $\quad 5(24 \%$ Patients also had disease progression; poor per oral uptake or myelosuppression likely $16(76 \%)$

Thrombocytopenia (<160 K/mcl)

Bleeding

Required treatment

$11(52 \%)$

Symptoms could be attributed to other factors (bevacizumab administration

$3(14 \%)$

anticoagulation therapy, higuted to other factors (bevacizumab administration, irritation)

Leucopenia $(<4 \mathrm{~K} / \mathrm{mcl})$ and/or neutropenia $(<1.5 \mathrm{~K} / \mathrm{mcl})$ Bacterial infections (urinary tract infection, 6 months post-RAE) $1(5 \%)$
Leucopenia required treatment with bone marrow stimulants (pegfilgrastim $6 \mathrm{mg} \mathrm{s} / \mathrm{c}$ or filgrastim $480 \mathrm{mcg} s / \mathrm{c}$ )

Required break from systemic chemotherapy due low blood count values (at 4-6 months post- $4(19 \%)$ RAE, break was for $1-2$ weeks)

Normal laboratory values $4(19 \%)$

No laboratory values data available as patients were lost to follow-up 
Radiation Segmentectomy for

Hepatic Metastases with ${ }^{90} \mathrm{Y}$

Glass-Based Microspheres:

Technical Considerations,

Correlations to Dosimetry

and Preliminary Results

Kurilova I., Bendet A., Fung E.K., Humm J.L., Do R.K.G., Boas F.E., Yarmohammadi H., Ziv E., Deipolyi A., Petre E.N., D’ Angelica M.I., Crane C.H., Kingham P.T, Cercek A., Beets-Tan R.G.H., Sofocleous C.T. 


\section{ABSTRACT}

Objectives

To evaluate safety and efficacy of ablative-dose ${ }^{\circ \circ}$ glass-based microspheres radiation segmentectomy (RS) in patients with limited metastatic liver disease not amenable to surgery or percutaneous ablation.

\section{Materials and methods}

Patients with $\leq_{3}$ tumors treated with RS from 6/2015-12/2017 were included. Tumor response, local tumor progression (LTP) and LTP-free survival (LTPFS) were defined using Choi and RECIST 1.1 criteria. Toxicities were evaluated using modified SIR criteria.

Results

Ten patients with 14 tumors underwent 12 procedures. Median tumor size was $3 \mathrm{~cm}$ (range, 1.4-5.6). Median follow-up was 8.8 months (range, 1.6-14.7). Response rates per Choi and RECIST 1.1 criteria were $8 / 8(100 \%)$ and $4 / 9(44 \%)$, respectively. Target tumor LTP rate was $1 / 14$ ( $7 \%$ ) resulting in 1-year LTPFS of $89 \%$ ( $95 \%$ CI, $43-98 \%$ ). New tumors occurred after 1/12 (8\%) procedures. Intra-patient dosimetry varied among partition model, MIRD, ${ }^{99 m} \mathrm{~m} \mathrm{C}-\mathrm{MAA}$ and Bremsstrahlung-based approaches with median tumor radiation dose of $650 \mathrm{~Gy}, 293 \mathrm{~Gy}, 202$ Gy and $112 \mathrm{~Gy}$, respectively. Median minimum tumor radiation dose was $67 \mathrm{~Gy}$ (range, 28-378) based on Bremsstrahlung SPECT/CT. A higher segment diseaseprogression rate was associated with lower radiation doses $(p=0.036, O R=0.96)$. One major complication was recorded in a patient post-Whipple who suffered anaphylactic reaction to prophylactic cefotetan and liver abscess in the RS region 6.5 months postprocedure. All patients were alive on last follow-up.

\section{Conclusion}

RS of $\leq 3$ hepatic segments can safely provide good local tumor control in selected patients with limited metastatic liver disease and treatment options. Optimal dosimetry methodology requires further investigation.

\section{INTRODUCTION}

Radiation segmentectomy (RS) is an intra-arterial therapy that selectively delivers very high ablative radiation dose (>190 Gy) to liver segments containing tumor [1-3]. This technique is intended to achieve complete tumor eradication in selected patients with limited liver tumor burden that cannot be treated by surgery or ablation $[1,2,4]$.

RS has been successfully used for the treatment of selected patients with localized hepatocellular carcinoma (HCC) that could not be resected or percutaneously ablated $[1,2,4-6]$. RS enabled to achieve significantly better local tumor control compared to other intra-arterial therapies (transarterial chemoembolization) [7-9]. RS resulted in $>90 \%$ necrosis rates, with complete necrosis rate of $52 \%$ [2], demonstrating outcomes similar to those of other local curative treatments [10].

Radiation segmentectomy allows the creation of treatment margins around the target tumor, similarly to surgical resection, percutaneous ablation or stereotactic ablative radiotherapy. This is the result of treating the entire segment containing the tumor (rather than superselective tumor targeting) and the tumor penetration by the $\beta$-emission $(\sim 1 \mathrm{~cm})$ around each microsphere loaded into the tumor and surrounding capillaries [11].

To date, most reports of ablative dose radioembolization involve the delivery of ${ }^{90} \mathrm{Y}$ glass-based microspheres $[1,2]$. Due to the high radioactivity per sphere, ${ }^{\circ} \mathrm{Y}$ glass-based microspheres deliver extremely high radiation dose into the targeted tumor(s) with relatively small number of spheres and minimal risk of stasis or occlusion even in relatively hypovascular tumors [2].

Worldwide secondary liver malignancies are more prevalent than primary liver tumors [12] with local therapy demonstrating potential for prolonged survival for this patient population $[13,14]$. There is very limited data on RS role as a local potentially curative therapy in patients with limited hepatic metastases [15].

This work was conducted to assess the feasibility, safety and efficacy of radiation segmentectomy in the setting of limited metastatic liver disease in patients who could not undergo surgery or percutaneous ablation. 


\section{MATERIALS AND METHODS}

Study population

Internal review board approval was obtained for this retrospective cohort study of ${ }^{90} \mathrm{Y}$ RS for liver metastases. Informed consent was not required for this type of study. All patients signed clinical informed consent.

All patients treated with RS for liver metastases between 6/2015 and 12/2017 were eligible for inclusion in the study.

Inclusion and exclusion criteria and rationale for radiation segmentectomy

Patients were eligible for RS if they had up to 3 unresectable liver metastases, not amenable to percutaneous ablation with sufficient ablation margins due to the following reasons: $\geq 3 \mathrm{~cm}$ tumor size, challenging tumor location near major hepatic structures or increased risk of adjacent organ damage. The patients also needed to meet standard ${ }^{\circ} \mathrm{Y}$ radioembolization eligibility criteria. RS was preferred to stereotactic ablative radiotherapy either by the patients (to avoid multiple radiation sessions) or by the treating physician (due to the challenging tumor position, requiring fiducial placement or near an organ at risk for collateral radiation injury) [16]

Study exclusion criteria and pre-procedural work-up were analogic to ${ }^{90} \mathrm{Y}$ radioembolization and are described in Table $\mathbf{1}$

Study objectives and definitions

Primary study objective was to assess the feasibility and efficacy of ${ }^{\circ} Y \mathrm{RS}$ in patients with limited metastatic disease (objective response rate, local tumor and liver progressionfree survivals).

Objective response rate (defined as complete and partial response rate) was assessed by an experienced radiologist (A.Bendet, faculty body imaging, 9 years of experience) based on CT imaging using Choi and RECIST 1.1 criteria (Supplement 1). Choi criteria were chosen as they have shown to correlate with overall survival following ${ }^{\circ} \mathrm{Y}$ radioembolization [17-19]. Tumor response rates were evaluated at first and second imaging follow-up time points after RS (at 4-8 and 12-16 weeks, respectively). Tumor attenuation was measured on portal venous phase with ROI comprising the tumor. In case of discordant findings, Choi criteria were considered superior to RECIST 1.1 [20].
Table 1 Radiation segmentectomy inclusion and exclusion criteria, pre-procedure work-up and angiographic mapping.

\begin{tabular}{|c|c|}
\hline Parameter & Description \\
\hline \multirow[t]{2}{*}{ Inclusion criteria } & $\begin{array}{l}\text { Radiation segmentectomy specific: } \\
\text { Up to } 3 \text { hepatic metastases } \\
\text { Ineligibility for liver surgery } \\
\text { Ineligibility for curative image-guided percutaneous ablation with margins (due to tumor } \\
\text { abutment of a major vessel and/or bile duct; increased risk of diaphragmatic injury (in } \\
\text { subcapsular dome lesions) or surrounding organ damage, that could not be protected by } \\
\text { hydrodissection; relatively large tumor size }(>3 \mathrm{~cm} \text { ) that would not allow the creation of } \\
\text { sufficient minimal ablation margins of at leass } 5 \mathrm{~mm} \text { ) } \\
\text { Local tumor progression post-ablation with suspicion of micrometastatic spread in the same } \\
\text { hepatic segment, but with no evidence of distant liver or other disease }\end{array}$ \\
\hline & 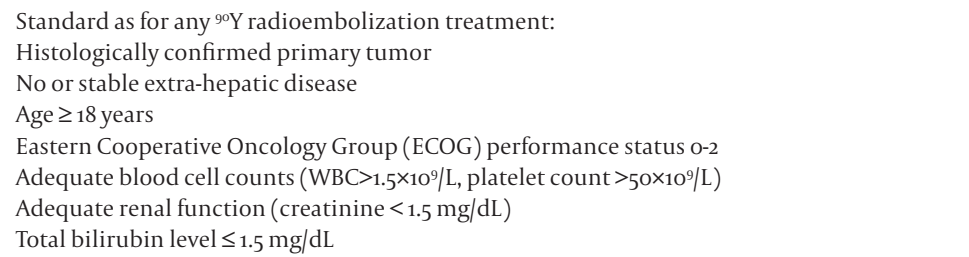 \\
\hline $\begin{array}{l}\text { Exclusion criteria } \\
\text { (analogic to } \\
\text { radioembolization) }\end{array}$ & $\begin{array}{l}\text { Prior hepatic radiotherapy } \\
\text { Evidence of severe cirrhosis } \\
\text { Severe portal hypertension } \\
\text { Uncorrectable flow to the gastrointestinal tract and/or }>20 \% \text { shunting to the lungs, as } \\
\text { determined by } 99 m \text { TC-MAA hepatic arterial perfusion scintigram (mapping) }\end{array}$ \\
\hline Pre-procedural work-up & $\begin{array}{l}\text { All patients were evaluated at a clinic visit within } 30 \text {-days before RS. Past medical history was } \\
\text { reviewed, physical examination was performed and relevant baseline laboratory values were } \\
\text { evaluated } \\
\text { Pre-procedural baseline imaging with liver dynamic (ideally triphasic) CT or contrast- } \\
\text { enhanced MRI and//or '\$rF-FDG PET/CT was available/ obtained within } 30 \text {-days from RS for } \\
\text { accurate re-staging of disease }\end{array}$ \\
\hline Angiographic mapping & 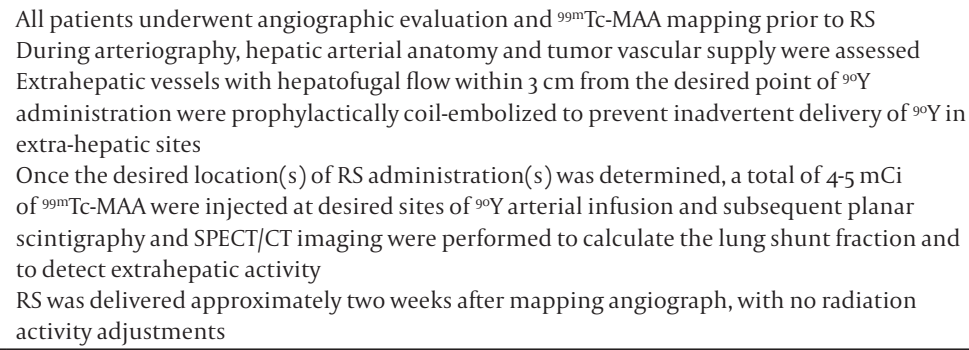 \\
\hline
\end{tabular}

Local tumor progression (LTP) was defined as progression of the target tumor and within $1 \mathrm{~cm}$ area around it (accounting for maximal $\beta$-emission penetration depth) [11]. Occurrence of new metastases within the treated segment was reported separately.

Secondary study objective was to assess the safety of RS. Toxicities were described within 6-months post-treatment according to modified SIR criteria [21, 22] with side effects reported separately. Side effects were defined as expected, undesired and 
frequent consequences of the procedure that were self-limiting without requirement of admission or change in care management [22].

\section{Methods to estimate the delivered radiation dose}

Ablative radiation dose (>190 Gy) was intended to be delivered in the target tumor and cause complete tumor destruction [2]. This was achieved via the intra-arterial delivery of ${ }^{9} Y$ Y glass-based microspheres (TheraSphere; MDS Nordion, Ottawa, Ontario, Canada) superselectively within the tumor-containing liver segment(s).

To measure radiation dose delivered to the tumor, volumetric segmentations of regions of interest (ROI) of the target tumors and the intended-to-treat Couinaud liver segments were performed on pre-treatment diagnostic contrast-enhanced CT scans (obtained within 6-weeks from RS) using Hermes Hybrid Viewer software (Hermes Medical Solutions, Stockholm, Sweden).

Radiation dose delivered to the tumor was estimated using three different methods (Table 2):

1) The manufacturer specified/simplified dose formula that is based on the Medical Internal Radiation Dose (MIRD) approach, taking into account lung shunt fraction and residual vial radiation activity [23]. This formula does not take into account preferential blood flow to the tumor during RS and assumes uniform microspheres distribution within the treated region;

2) Image-based dosimetry approaches, that take into account non-uniform particles distribution. These included ${ }^{99 m}$ Tc-MAA SPECT/CT-based and Bremsstrahlung SPECT/CT-based dosimetry approaches. Imaging-based dosimetry methods were applied only if rigid imaging co-registration was possible to account for differences in patient positioning and respiratory motion. This was feasible in $4 / 10$ (40\%) of the study patients.

3) Partition model-based ${ }^{99 m}$ Tc-MAA SPECT predictive dosimetry. This model is more accurate and personalized but more complex than semi-empirical methods [24]. It is based on three compartments dosimetry (tumor, normal liver and lung) with deposited activity determined based on ${ }^{99 m}$ Tc-MAA SPECT/CT.

\section{Statistical analysis}

The median follow-up period was calculated for the patients who were alive on last follow-up. Survivals (overall, liver and extrahepatic disease progression-free) were estimated using Kaplan-Meier methodology. Statistical analysis was performed using STATA 11.o statistical software (StataCorp LLC, USA). P < 0.05 was considered significant.
Table 2 Calculation of radiation dose delivered to segment/tumo

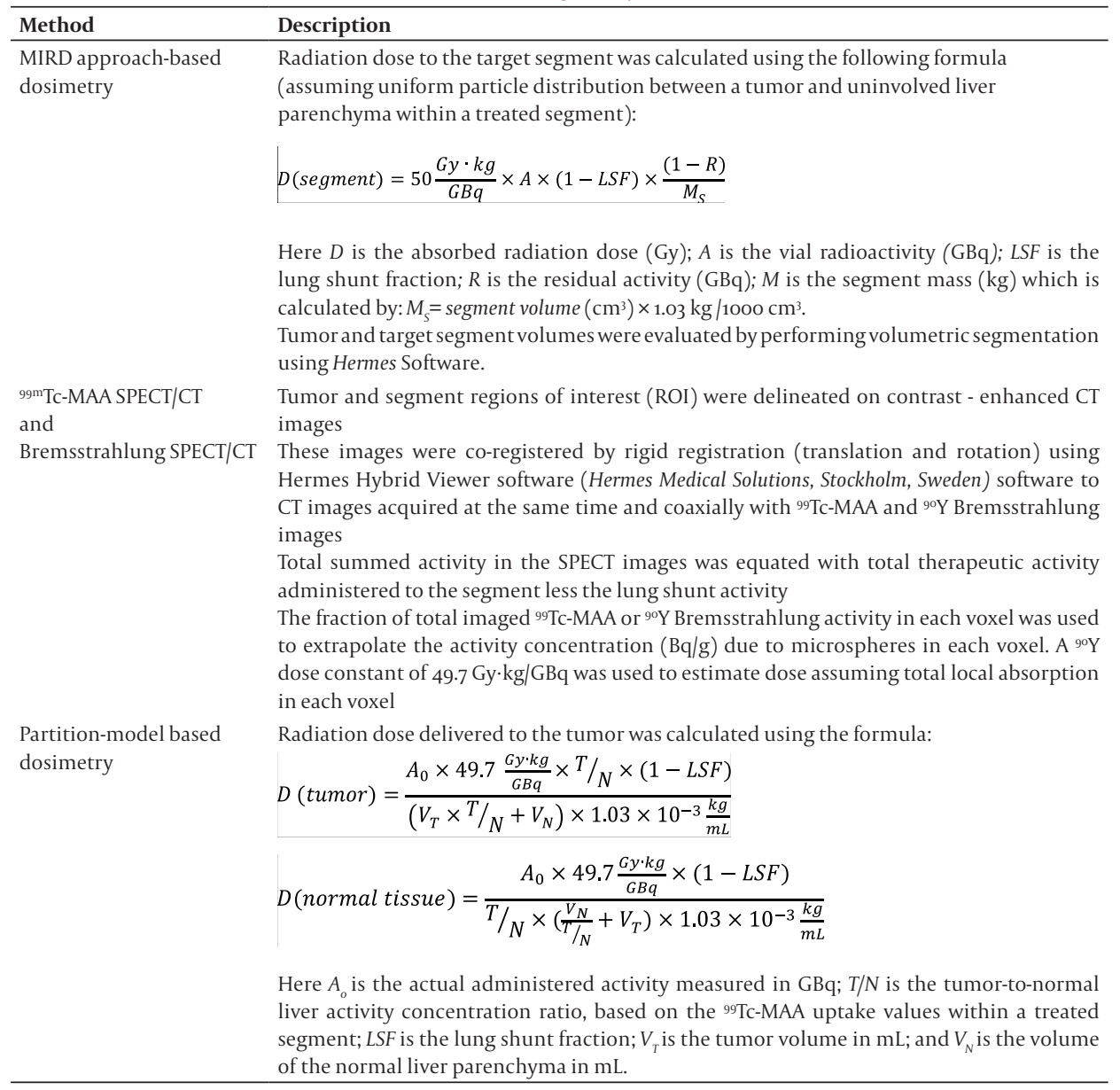

\section{RESULTS}

Patient, tumor and treatment characteristics

We treated 10 patients with 14 tumors 12 RS sessions. Incidence of mono-, bi- and trisegmentectomies (anterior sector + Segment 3 or 4 ) was: 4/10 (40\%), 4/10 (40\%) and 2/10 (20\%). Total of 3/10 of the patients had more than one tumor located in separate lobes; in $2 / 3$ of these cases RS was completed in 2 separate sessions (5-10 weeks apart). The entire prescribed radioactivity was delivered in all cases with no stasis in any of the RS sessions. Detailed patient, tumor and treatment characteristics are depicted in Table 3. Representative RS case is depicted in Figure 1 


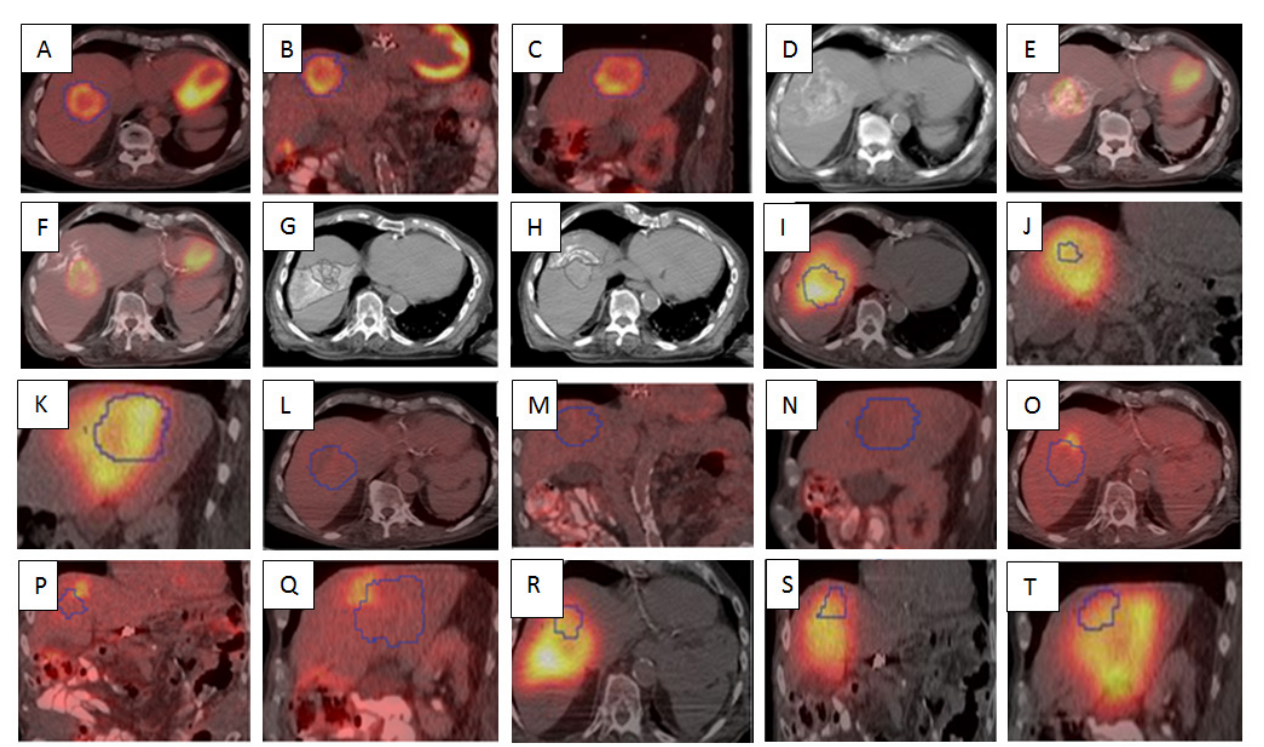

Figure 1

A-C, Pre-treatment ${ }^{18} \mathrm{~F}$-FDG PET/CT showed FDG-avid liver metastasis, $\mathrm{SUV}_{\text {max }}=8.6, \mathrm{SUV}_{\text {mean }}$ of background liver is 2.4 in patient with colorectal cancer;

D, The fused image of CT angiography (CTA) of Segment 5 branch and CTA of Segment 8 branch;

E-F, Fusion of pre-treatment liver ${ }^{18} \mathrm{~F}-\mathrm{FDG}$ PET/CT scan (showing the FDG uptake by the tumor) with the liver CTA of $\mathrm{S}_{5}$ and $\mathrm{S} 8$ branches. The fused images demonstrated that Segment 5 branch almost entirely supplied the tumor:

G, Segmentation of the tumor and the normal liver parenchyma supplied by Segment 5 branch.

$\mathbf{H}$, Segmentation of the tumor and the normal liver parenchyma supplied by Segment 8 branch. Tumor volume planned to be covered by Segment 5 injection was $107 \mathrm{~cm}^{3}(74 \%)$. Tumor volume planned to be covered by Segment 8 injection was $38 \mathrm{~cm}^{3}(26 \%)$. Segment 5 branch perfused liver volume - 286 $\mathrm{cm}^{3}$, Segment 8 branch perfused liver volume - $156 \mathrm{~cm}^{3}$. Total liver volume planned to irradiate during radiation segmentectomy was $442 \mathrm{~cm}^{3}$ (33\% of total liver volume);

I-K, Bremsstrahlung SPECT/CT showed deposition of 9॰Y with the delineated metabolic tumor volume, defined on ${ }^{18} \mathrm{~F}$-FDG PET/CT;

L-N, Complete metabolic resolution of tumor FDG-uptake on first follow-up;

O-Q Local tumor progression on second follow-up ${ }^{8} \mathrm{~F}$-FDG PET/CT with metabolic tumor volume, defined on ${ }^{8} \mathrm{~F}-\mathrm{FDG}$ PET/CT before treatment is marked in blue;

R-T, Correlation of area of local tumor progression with distribution and deposition of ${ }^{\circ} \mathrm{Y}$ particles as seen on Bremsstrahlung SPECT/CT.
Table 3 Patient, tumor and treatment characteristics

\begin{tabular}{|c|c|}
\hline Parameter & Result \\
\hline Number of patients & 10 \\
\hline Number of tumours & 14 \\
\hline Number of procedures & 12 \\
\hline \multicolumn{2}{|l|}{ Origin of metastases: } \\
\hline colorectal & 2 \\
\hline breast & \\
\hline ovarian & 1 \\
\hline non-small cell lung cancer & 1 \\
\hline pancreatic & 1 \\
\hline gallbladder & 1 \\
\hline gastric & \\
\hline ampullary & 1 \\
\hline gastro-oesophageal junction & 1 \\
\hline Patients age, mean & $68.1 \pm 13.6$ years \\
\hline Gender & $60 \%$ woman \\
\hline Median follow-up, range & 8.8 months $(1.6-14.7)$ \\
\hline \multicolumn{2}{|l|}{ Reason for radiation segmentectomy } \\
\hline Lesion too large to ablate & $8 / 14(57 \%)$ \\
\hline Abutment of major liver vascular and biliary structures & $2 / 14(14 \%)$ \\
\hline Subcapsular location with increased risk of diaphragmatic injury & $2 / 14(14 \%)$ \\
\hline $\begin{array}{l}\text { Increased risk of extrahepatic structures injury (heart, phrenic nerve, stomach, } \\
\text { superior mesenteric and gastroduodenal arteries) }\end{array}$ & $2 / 14(14 \%)$ \\
\hline \multicolumn{2}{|l|}{ Prior liver-directed therapies } \\
\hline Yes (thermal ablation, liver resection, liver bland embolization) & $4 / 10(40 \%)$ \\
\hline No & $6 / 10(60 \%)$ \\
\hline \multicolumn{2}{|l|}{ Number of treated segments } \\
\hline One & $4 / 10(40 \%)$ \\
\hline Two & $4 / 10(40 \%)$ \\
\hline Three (Segments 5,8 + Segment 3 or Segments 5,8 + Segment 4) & $2 / 10(20 \%)$ \\
\hline Number of tumours treated per patient & 1-3 tumours \\
\hline \multicolumn{2}{|l|}{ Number of ${ }^{\circ} \mathrm{Y}$ infusions } \\
\hline One $^{*}$ & 9/14(64\%) \\
\hline Two (tumours were supplied by two hepatic artery branches) & $5 / 14(36 \%)$ \\
\hline Median tumor size, range** & $3 \mathrm{~cm}(1.4-5.6)$ \\
\hline \multicolumn{2}{|l|}{ Baseline extrahepatic disease } \\
\hline Yes & $2 / 10(20 \%)$ \\
\hline No & $8 / 10(80 \%)$ \\
\hline
\end{tabular}

Response evaluations: first and second imaging assessments after RS

Eight/10 (80\%) of patients were evaluated by triphasic CT scan and all demonstrated partial $(n=7)$ or complete response $(n=1)$ according to Choi criteria, resulting in $100 \%$ objective response rate on first and second imaging follow-up (at 4-8 and 12-16 weeks after RS, respectively). One patient with only MRI available at baseline had complete response on the first imaging 4-months post-RS (based on RECIST 1.1). One patient had only ${ }^{18} \mathrm{~F}-\mathrm{FDG}$ PET/CT imaging available post-RS, showing stable disease (14\% decrease in 
SUV $_{\max }$, based on EORTC criteria). Objective response rate based by RECIST 1.1 criteria was $4 / 9(44 \%)$.

\section{LTP rate and LTPFS}

One-year LTPFS (for target tumors) was 89\% (95\%CI, 43\%-98\%) with median LTPFS not reached (Figure 2)

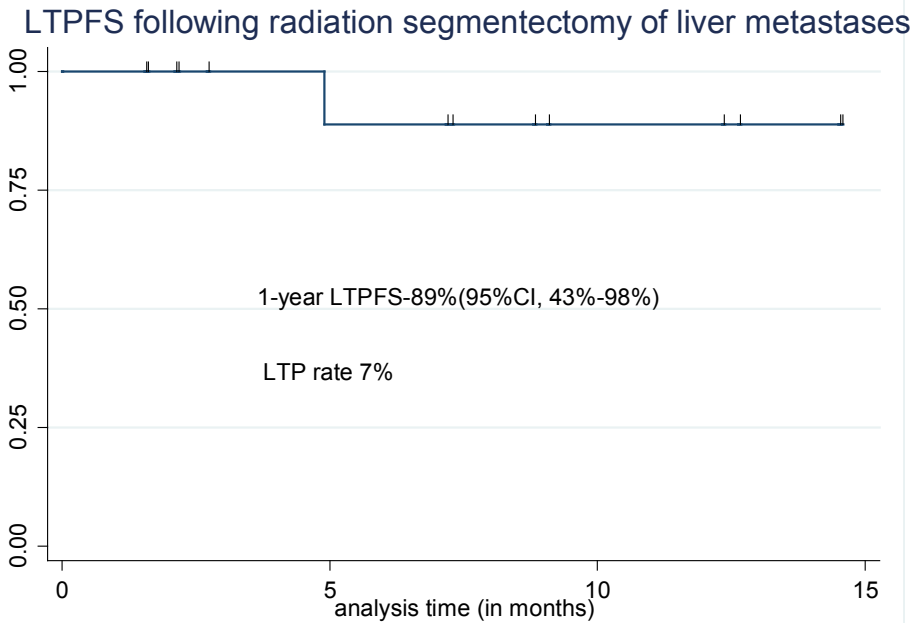

Figure 2 Local tumor progression-free survival (LTPFS) after radiation segmentectomy. Target tumor LTP rate was $7 \%$ with 1-year LTPFS of $89 \%$

There was only one LTP at 4.9 months post-RS in a patient with colorectal liver metastasis (Figure 1). This was a marginal recurrence of the largest tumor in this cohort $(>5 \mathrm{~cm}$ in size). Only $35 \%$ of this tumor received the intended radiation dose $>200 \mathrm{~Gy}$. A minimum tumor radiation dose of $100 \mathrm{~Gy}$ was calculated based on 99mTc-MAA SPECT/CT. A relatively poor coverage of the tumor was shown in post-RS Bremsstrahlung imaging (Figure 1 I-K) and corresponded to the area of LTP (Figure 1 $\mathbf{O}-\mathbf{Q}$ ). The same patient developed disease progression in the liver beyond the treated segments. Additional locoregional therapies were considered technically challenging due to recurrent tumor location, patient's age, comorbidities and worsening performance status. Thus the patient received palliative oral systemic chemotherapy only.

In another patient, a new metastasis distant to the target tumor, but within the treated segment was detected 9.1 months post-RS. This new tumor was treated with MW ablation. This patient had prior history of multiple liver-directed therapies in other liver segments than the one treated with RS.

\section{Dosimetry}

Median radiation dose delivered to treated segment(s), tumor(s) and lungs, total treated tumor volume and percentage of irradiated liver are described in Table 4.

There were large intra-patient dosimetry distribution variations among the MIRD, partition model, ${ }^{99 m} \mathrm{TC}-\mathrm{MAA}$ SPECT/CT and Bremsstrahlung SPECT/CT-based dosimetry, with the latter demonstrating the lowest absorbed radiation doses.

Median radiation doses delivered to the tumor based on partition model, MIRD approach, 99m Tc-MAA and Bremsstrahlung SPECT/CT were 650 Gy, 293 Gy, 202 Gy and 112 Gy, respectively. Median minimum tumor radiation dose was 54 Gy (range, o-10o Gy, based on ${ }^{99 m} \mathrm{TC}-\mathrm{MAA}$ SPECT/CT) and $67 \mathrm{~Gy}$ (range, 28-378 Gy, based on Bremsstrahlung SPECT/CT). Median radiation doses delivered to the target segments were $227 \mathrm{~Gy}, 293 \mathrm{~Gy}$, 98 Gy and 47 Gy, respectively (Table 4).

\section{Dose-response correlatio}

A relatively higher disease progression rate in the treated segment (of the target tumor or occurrence of new tumors distant from the target tumor) was significantly associated with lower radiation dose delivered to that segment, according to the MIRD approachbased dosimetry $(\mathrm{p}=0.036, \mathrm{OR}=0.96)$. The correlation of the absorbed radiation dose in the treated segment (based on MIRD approach) with the corresponding segment progression-free survival was not significant $(\mathrm{p}=0.3, \mathrm{OR}=0.98)$.

Distant intrahepatic disease progression

4/10 (40\%) patients developed disease progression in the liver, outside the RS field. Median untreated liver progression-free survival was 4.9 months ( $95 \% \mathrm{CI}, 2.1-\mathrm{NR}$ ).

Toxicity

Side effects were reported in half of the procedures (Table 5). All side effects were selflimiting without requirement for admission or change in care management.

Minor complications occurred after $1 / 12(8 \%)$ (95\% CI, O-23\%) of RS procedures. This involved abdominal pain, fever, hypertension and atrial fibrillation (in a patient with history of atrial fibrillation). This required overnight observation and was successfully managed without further sequelae or additional treatment. 


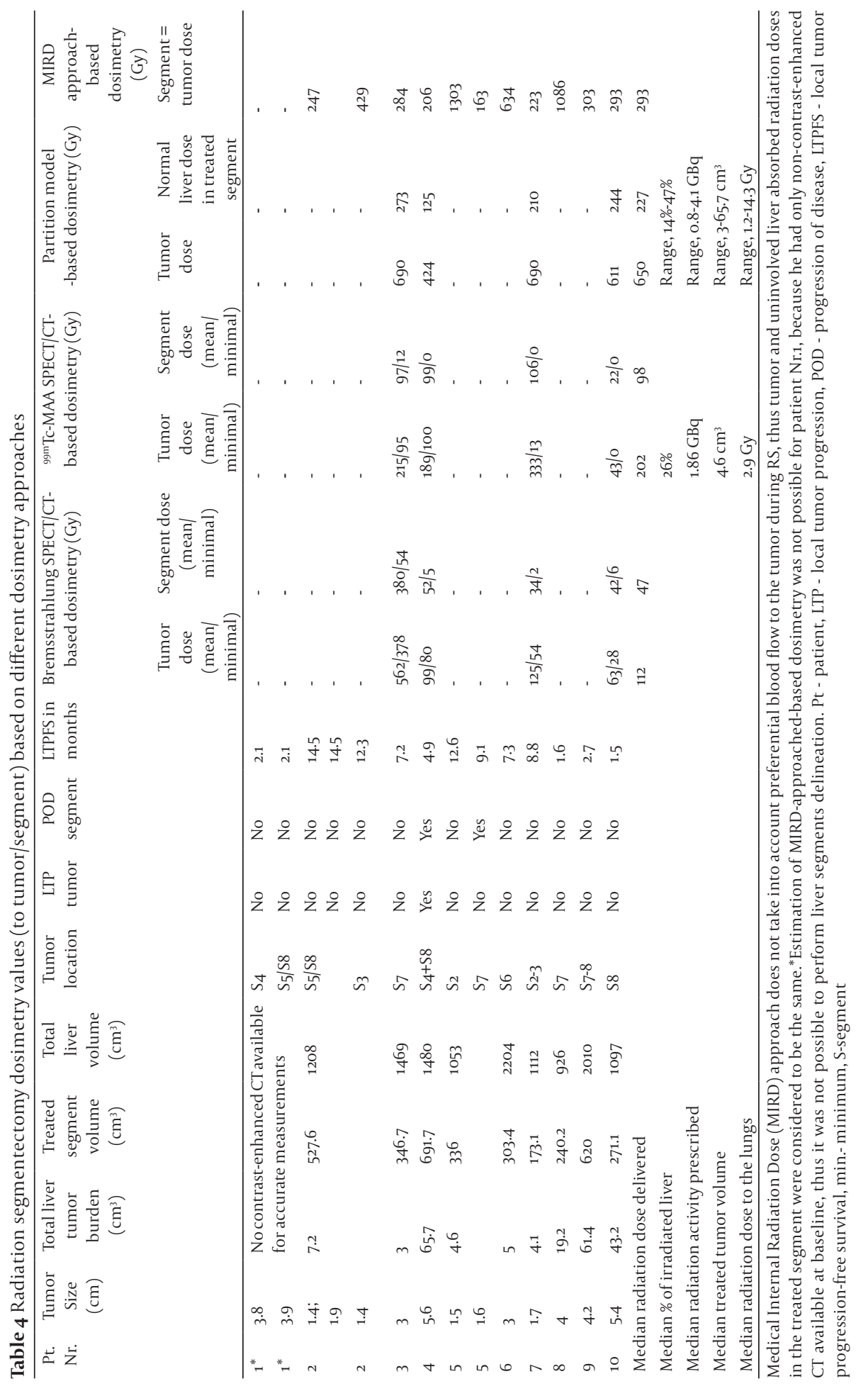

Table 5 Side effects following radiation segmentectomy

\begin{tabular}{|c|c|}
\hline Side effects & Incidence \\
\hline Pronounced fatigue & $4 / 12(33 \%)$ \\
\hline Abdominal pain/distension & $3 / 12(25 \%)$ \\
\hline Nausea & $1 / 12(8 \%)$ \\
\hline Dyspnea & $1 / 12(8 \%)$ \\
\hline Weight loss ( $\geq 10$ pounds) & $1 / 12(8 \%)$ \\
\hline Elevation of hemidiaphragm including phrenic artery, resolved on its own & $1 / 12(8 \%)$ \\
\hline Total patients number & $6 / 12(50 \%)^{*}$ \\
\hline
\end{tabular}

Major complications occurred after 1/12 (8\%) (95\%CI, o-23\%) of RS procedures in a high-risk patient with history of Whipple procedure 4-years prior to RS. This patient developed anaphylaxis to prophylactic cefotetan. The RS was aborted and performed a month later, using premedication with clindamycin, gentamycin and dexamethasone. The patient did not receive additional antibiotics after RS. One month after RS he underwent MW ablation for a liver tumor outside the RS field that was complicated by perihepatic abscess, requiring percutaneous drainage. The same patient presented 5-months after RS with bilomas within the RS region, complicated by polymicrobial liver abscess, requiring 9-days of hospitalization for abscess aspiration and intravenous antibiotics with no further sequelae.

\section{DISCUSSION}

Technical considerations

In this study we described our initial experience with RS in patients with liver metastases. RS was utilized for selected patients with oligometastatic disease and limited treatment options. This included patients not amenable to resection or percutaneous ablation. The main RS treatment intent was local cure, similarly to ablative external radiation therapy [25] or ablation [26]. During RS a target ablative radiation dose of $>190$ Gy was administered superselectively into the tumor containing liver segments. The choice of this radiation dose was based on published data for RS in the patients with HCC, which reported a correlation of delivered radiation dose to the level of necrosis in explanted tumors [2].

Even though currently RS has been reported only for the treatment of up to two liver segments, we described successful RS for up to three segments (in two sessions). We intentionally used glass-based instead of resin microspheres during RS to enable 
maximal radiation dose delivery with the smallest possible number of spheres and risk of stasis [12, 27-30].

\section{Efficacy}

Despite the heterogeneous population treated in this cohort, encouraging local tumor control was achieved. The overall 100\% objective response rate by Choi criteria compared favorably to $81 \%$ response rate reported after RS in HCC patients [1]. The LTP rate of $7 \%$ and resulting 1-year LTPFS of $89 \%$ of the target tumors need to be assessed in the context of patients with advanced and refractory metastatic disease that had very limited if any local curative treatment options.

The single marginal LTP occurred in the largest tumor within the cohort ( $>5 \mathrm{~cm}$ in size), that only partially received the intended $>200$ Gy radiation dose in $35 \%$ of the target tumor volume. The same tumor received a minimum tumor radiation dose as low as 100 Gy. Of interest the area of LTP corresponded with area of predicted lower radiation dose by ${ }^{99 m}$ Tc MAA SPECT method and the actual lower dose exposure documented in the postRS Bremsstrahlung SPECT. This is similar to prior research, where incomplete tumor targeting was associated with worse local tumor control following ${ }^{\circ} Y$ radioembolization [31]. These findings indicating areas of technical weaknesses that can be improved for the optimization of this technique and the sufficient radiation dose delivery to target tumor and surrounding parenchyma.

\section{Safety}

Self-limiting side effects were noted after a half of RS procedures and were similar to known side effects of ${ }^{\circ} \mathrm{Y}$ radioembolization (weakness, nausea, abdominal distention, weight loss and transient dyspnea). None of these required admission or medication [29, 32]. Major complication was noted in a single patient, who was high-risk in developing complications: it is well known that patients with Whipple or prior sphincterotomy and in general any biliary intervention are high risk for liver abscess formation after any liver-directed therapies [33-36]. It is therefore recommended that they also be considered as high risk for radioembolization and be protected with biliary secreted antibiotics in the peri- and post-procedure period. All patients were alive on last follow-up without evidence of radiation-related toxicity. Similarly, Riaz et al. reported favorable adverse event profile following RS for HCC patients [1]. This suggests that RS is safe as expected since the non-exposed liver remains fully functional.

Dosimetry

We reported large variation in estimated absorbed radiation doses among different dosimetry assessment methods used.

The partition model-based dosimetry theoretically should have provided the most accurate tumor radiation dose values amongst the three methods employed in this study. It accounts for a preferential flow of microspheres to tumor and for the entire delivered radiation activity. However, it also has several limitations: it assumes, that radiation dose is homogeneously distributed in each compartment (tumor, uninvolved liver parenchyma and the lungs) [37, 38], which is incorrect; and it is based on the distribution of 99mTc-MAA, rather than ${ }^{90} \mathrm{Y}$ particles [39-42].

BremsstrahlungSPECT/CT dose estimates might be unreliable due to the lack of consensus on the correct settings for energy windows and corrections [43]. The MIRD-based segment dosimetry estimation is also not optimal as it assumes uniform distribution of radiation activity within the tumor and the non-involved liver parenchyma (tumor-tonormal liver ratio equal to 1 ), that is not accurate [44].

The discrepancy among the methods is concordant with prior data indicating, that current dosimetry calculators are not optimal and represent gross oversimplifications of tumor architecture and radiobiology. Alternatively, three-dimensional voxel-based dosimetry using ${ }^{\circ} \mathrm{Y} \mathrm{PET} / \mathrm{CT}$ was found to be beneficial, detecting associations of tumor response, progression-free, overall survival and toxicity with the delivered ${ }^{90} \mathrm{Y}$ dose [31]. However, it cannot be used for treatment planning and dose delivery predictions. An optimal radiation activity calculation methodology for RS must be applicable to treatment planning, accounting for microspheres' number and distribution as well as tumor type and characteristics (vascularity, perfusion and genetic factors). Radiologypathology correlations with dosimetry are very much warranted in an effort to better understand and optimize radioembolization as a treatment for cancer.

\section{Dose-response correlations}

Despite the small number of patients included in the study, we found a significant correlation between the absorbed radiation dose in the treated segment (based on MIRD dosimetry) and the incidence of disease progression rates, implying greater tumor control with increased radiation dose to the target segment. Subsequent correlation of increased radiation dose absorbed in the treated segment with segment progression-free survival was not significant. While the disease progression rate result is promising, progression-free survival is generally considered a better indicator of local tumor control than disease progression rate. The lack of significant correlation between radiation dose delivered to the segment and segment progression-free survival in this 
study is most likely the result of the small cohort and the pathologic heterogeneity of the treated metastases.

\section{Study limitations}

This study has several limitations including the heterogeneous patient and tumor characteristics, the retrospective single-center nature, the small sample size, the relatively short follow-up time and the lack of uniform follow-up imaging. These limitations are inherent to the limited population of patients with metastatic disease that were candidates and received RS to date.

The initial experience with RS for hepatic metastases described in this short report is encouraging and sets the stage for further investigation regarding RS as a potential alternative local curative treatment for hepatic metastases. Well-designed prospective investigations with pathologic validation of response and correlations to dosimetry are very much warranted to assess radiation segmentectomy in the treatment of metastatic liver disease.

\section{CONCLUSION}

This study provided the data on initial single-center experience with radiation segmentectomy in selected patients with limited hepatic metastases that could not be treated with resection or ablation. Specifically, the study demonstrated that radiation segmentectomy of up to three hepatic segments is feasible and safe providing good local tumor control in patients with limited liver metastatic disease and poor if any treatment options.

\section{REFERENCES}

1. Riaz, A., et al., Radiation segmentectomy: a novel approach to increase safety and efficacy of radioembolization. Int J Radiat Oncol Biol Phys, 2011. 79(1): p. 163-71.

2. Vouche, M., et al., Unresectable solitary hepatocellular carcinoma not amenable to radiofrequency ablation: multicenter radiologypathology correlation and survival of radiation segmentectomy. Hepatology, 2014. 6o(1): p. 192-201.

3. Lewandowski, R.J., et al., Radiation Segmentectomy: Potential Curative Therapy for Early Hepatocellular Carcinoma. Radiology, 2018: p. 171768.

4. Biederman, D.M., et al., Radiation Segmentectomy versus TACE Combined with Microwave Ablation for Unresectable Solitary Hepatocellular Carcinoma Up to $3 \mathrm{~cm}$ : A Propensity Score Matching Study. Radiology, 2017. 283(3): p. 895-905.

5. Padia, S.A., et al., Superselective yttrium-9o radioembolization for hepatocellular carcinoma yields high response rates with minimal toxicity. J Vasc Interv Radiol, 2014. 25(7): p. 1067-73.

6. Sofocleous, C.T. and F.E. Boas, Radiation Segmentectomy for Hepatocellular Carcinoma: Ready for Prime Time? Radiology, 2018: p. 180163.

7. Salem, R., et al., Y9o Radioembolization Significantly Prolongs Time to Progression Compared With Chemoembolization in Patients With Hepatocellular Carcinoma Gastroenterology, 2016. 151(6): p. 1155-1163.e2.

8. Padia, S.A., et al., Segmental Yttrium-go Radioembolization versus Segmental Chemoembolization for Localized Hepatocellular
Carcinoma: Results of a Single-Center, Retrospective, Propensity Score-Matched Study. J Vasc Interv Radiol, 2017. 28(6): p. 777-785.e1.

9. Biederman, D.M., et al., Radiation Segmentectomy versus Selective Chemoembolization in the Treatment of EarlyStage Hepatocellular Carcinoma. J Vasc Interv Radiol, 2018. 29(1): p. 30-37.e2.

10. Lewandowski, R.J., et al., Radiation Segmentectomy: Potential Curative Therapy for Early Hepatocellular Carcinoma. Radiology, 2018. 287(3): p. 1050-1058.

11. Burrill J., H.U., Liu D.M., Advances in radioembolization - Embolics and isotopes. Nuclear Medi Radiat Ther, (2011; 2:107).

12. Lewandowski, R.J., et al., goY microsphere (TheraSphere) treatment for unresectable colorectal cancer metastases of the liver: response to treatment at targeted doses of $135-$ 150 Gy as measured by [18F] fluorodeoxyglucose positron emission tomography and computed tomographic imaging. J Vasc Interv Radiol, 2005. 16(12): p. 1641-51.

13. Ruers, T., et al., Local Treatment of Unresectable Colorectal Liver Metastases: Results of a Randomized Phase II Trial. J Natl Cancer Inst, 2017. 109(9).

14. Creasy, J.M., et al., Actual 10-year survival after hepatic resection of colorectal liver metastases: what factors preclude cure? Surgery, 2018. 163(6): p. 1238-1244.

15. Meiers, C., et al., Safety and initial efficacy of radiation segmentectomy for the treatment of hepatic metastases. J Gastrointest Oncol, 2018. 9(2): p. 311-315.

16. Crane, C.H. and E.J. Koay, Solutions that enable 
ablative radiotherapy for large liver tumors: Fractionated dose painting, simultaneous integrated protection, motion management, and computed tomography image guidance. Cancer, 2016. 122(13): p. 1974-86

17. Weng, Z., et al., Choi criteria are superior in evaluating tumor response in patients treated with transarterial radioembolization for hepatocellular carcinoma. Oncol Lett, 2013. 6(6): p. 1707-1712.

18. Jongen, J.M.J., et al., Anatomic versus Metabolic Tumor Response Assessment after Radioembolization Treatment. J Vasc Intery Radiol, 2018. 29(2): p. 244-253.e2.

19. Shady, W., et al., Metabolic tumor volume and total lesion glycolysis on FDG-PET/CT can predict overall survival after $(90) Y$ radioembolization of colorectal liver metastases: A comparison with SUVmax, SUVpeak, and RECIST 1.o. Eur J Radiol, 2016. 85(6): p. 1224-31.

20. Shady, W., et al., Surrogate Imaging Biomarkers of Response of Colorectal Liver Metastases After Salvage Radioembolization Using goY-Loaded Resin Microspheres. AJR Am J Roentgenol, 2016. 207(3): p. 661-70.

21. Sacks, D., et al., Society of Interventional Radiology clinical practice guidelines. J Vasc Interv Radiol, 2003. 14(9 Pt 2): p. S199-202.

22. Salem, R., et al., Research reporting standards for radioembolization of hepatic malignancies. J Vasc Interv Radiol, 2011. 22(3): p. 265-78.

23. Salem, R.and K.G. Thurston,Radioembolization with goYttrium microspheres: a state-of-theart brachytherapy treatment for primary and secondary liver malignancies. Part 1: Technical and methodologic considerations. J Vasc Interv Radiol, 2006. 17(8): p. 1251-78.

24. Kao, Y.H., et al., Clinical implications of the body surface area method versus partition model dosimetry for yttrium-go radioembolization using resin microspheres: a technical review. Ann Nucl Med, 2011. 25(7): p. 455-61.

25. He, X., et al., Curative-intent radiotherapy in patients with oligometastatic lesions from colorectal cancer: A single-center study. Medicine (Baltimore), 2018. 97(40): p. e12601. 26. Dupre, A., et al., Curative-intent treatment of recurrent colorectal liver metastases: A comparison between ablation and resection. Eur J Surg Oncol, 2017. 43(10): p. 1901-1907.

27. Hickey, R., et al., goY Radioembolization of Colorectal Hepatic Metastases Using Glass Microspheres: Safety and Survival Outcomes from a 531-Patient Multicenter Study. I Nucl Med, 2016. 57(5): p. 665-71.

28. Sofocleous, C.T., et al., Radioembolization as a Salvage Therapy for Heavily Pretreated Patients With Colorectal Cancer Liver Metastases: Factors That Affect Outcomes. Clin Colorectal Cancer, 2015. 14(4): p. 296-305

29. Kurilova, I., et al., Factors Affecting Oncologic Outcomes of goY Radioembolization of Heavily Pre-Treated Patients With Colon Cancer Liver Metastases. Clin Colorectal Cancer, 2019. 18(1): p. 8-18

30. Kurilova, I., et al., (90) Y Resin Microspheres Radioembolization for Colon Cancer Liver Metastases Using Full-Strength Contrast Material. Cardiovasc Intervent Radiol, 2018. 41(9): P. 1419-1427.

31. Allimant, C., et al., Tumor Targeting and ThreeDimensional Voxel-Based Dosimetry to Predict Tumor Response, Toxicity, and Survival after Yttrium-go Resin Microsphere Radioembolization in Hepatocellular Carcinoma. I Vasc Interv Radiol, 2018. 29(12): p. 1662-1670.e4.
32. Riaz, A., R. Awais, and R. Salem, Side effects of yttrium-9o radioembolization. Front Oncol, 2014. 4: p. 198

33. Shibata, T., et al., Cholangitis and liver absces after percutaneous ablation therapy for liver tumors: incidence and risk factors. J Vasc Interv Radiol, 2003. 14(12): p. 1535-42.

34. Elias, D., et al., Liver abscess after radiofrequency ablation of tumors in patients with a biliary tract procedure. Gastroenterol Clin Biol, 2006. 3o(67): p. 823-7.

35. Huang, S.Y., et al., Prevention and management of infectious complications of percutaneous interventions. Semin Intervent Radiol, 2015 32(2): p. 78-88.

36. Kim, W., et al., Risk factors for liver abscess formation after hepatic chemoembolization. I Vasc Interv Radiol, 2001. 12(8): p. 965-8.

37. Hogberg, J., et al., Heterogeneity of microsphere distribution in resected liver and tumour tissue following selective intrahepatic radiotherapy EJNMMI Res, 2014. 4(1): p. 48

38. Hemmingsson, J., et al., Autoradiography and biopsy measurements of a resected hepatocellular carcinoma treated with go yttrium radioembolization demonstrate large absorbed dose heterogeneities. Adv Radiat Oncol, 2018. 3(3): p. 439-446

39. Gnesin, S., et al., Partition Model-Based 99mTcMAA SPECT/CT Predictive Dosimetry Compared with goYTOF PET/CT Posttreatment Dosimetry in Radioembolization of HepatocellularCarcinoma: A Quantitative Agreement Comparison. I Nucl Med, 2016. 57(11): p. 1672-1678.

40. Wondergem, M., et al., 99mTc-macroaggregated albumin poorly predicts the intrahepatic distribution of goY resin microspheres in hepatic radioembolization. J Nucl Med, 2013. 54(8): p.
1294-301.

41. Ilhan, H., et al., Predictive Value of 99mTcMAA SPECT for goY-Labeled Resin Microsphere Distribution in Radioembolization of Primary and Secondary Hepatic Tumors. J Nucl Med, 2015. 56(11): p. 1654-60.

42. Haste, P., et al., Correlation of Technetium-99m Macroaggregated Albumin and Yttrium-go Glass Microsphere Biodistribution in Hepatocellular Carcinoma: A Retrospective Review of Pretreatment Single Photon Emission CT and Posttreatment Positron Emission Tomography/ CT. J Vasc Interv Radiol, 2017. 28(5): p. 722-730.

43. Elschot, M., et al., Quantitative comparison of PET and Bremsstrahlung SPECT for imaging the in vivo yttrium-go microsphere distribution after liver radioembolization. PLoS One, 2013. 8(2): p. e55742

44. Westcott, M.A., et al., The development, commercialization, and clinical context of yttrium-go radiolabeled resin and glass microspheres. Adv Radiat Oncol, 2016. 1(4): p. 351-364. 


\section{SUPPLEMENTARY MATERIAL}

Supplement 1 Response evaluation criteria used in the study Response Category $\quad$ RECIST 1.1 Guidelines \begin{tabular}{lll} 
& Choi Criteria \\
\hline Partial response & $\geq 30 \%$ decrease in sum of longest tumor & $\geq 15 \%$ decrease
\end{tabular} Choi Criteria $215 \%$ decrease in tumor attenuation (HU) or $\geq$

Stable disease No partial response or progressive disease. mor size. Progressive disease $\quad>20 \%$ increase in sum of longest tumor

(n)

Has $\geq 10 \%$ increase in tumor size, but does measured by change in tual response as development of new tumors. 


\section{PART 2}

Management of Colorectal Lung Metastases In Interventional Oncology Setting 


\section{Image-Guided Thermal}

Ablation for the Treatment of

Pulmonary Malignancies

Samdeep K. Mouli, Ieva Kurilova, Constantinos T. Sofocleous, and Robert J. Lewandowski

Published in:

American Journal of Roentgenology 2017 209:4, 740-751 


\section{ABSTRACT}

Objective

Image-guided thermal ablation is a minimally invasive treatment option for patients with primary and secondary pulmonary malignancies. Modalities include radiofrequency ablation, microwave ablation and cryoablation.

\section{Conclusion}

Although no large randomized studies exists comparing ablation to surgery or radiotherapy, numerous studies have reported safety and efficacy for the treatment of both primary and metastatic disease in select patients. Future studies will refine patient selection, procedural technique, and assessment for local recurrence and will evaluate long-term survival.
A variety of image-guided thermal ablation techniques exist for patients with non-small cell lung carcinoma (NSCLC) and oligometastatic disease. Many of these patients are not surgical candidates, so thermal ablation techniques-including radiofrequency ablation (RFA), microwave ablation (MWA), and cryoablation-have been explored, showing safety, efficacy, and good local disease control while preserving the lung parenchyma [1-8]. For patients with stage I or II NSCLC, surgical resection provides the best curative option, with 5-year survival rates of 50-75\% [9]. However, only one-third of patients may meet the criteria for lobar or sublobar resection [10]. For the remaining patients, ablative therapies have proved useful [3,11-14]. Additionally, the lungs are the second most common site of metastatic disease [15]. In selected patients with a limited number of tumors that can be ablated with adequate margins or those who present with residual or recurrent disease after having undergone other treatments, percutaneous thermal ablation plays a role in disease management. Over the past 2 decades, ablation has played an important role in the treatment of both primary and secondary pulmonary malignancies, providing an effective therapy, which can also provide potential cure in select cases.

\section{MECHANISM OF ACTION AND LOCAL EFFICACY}

The unique characteristics of pulmonary parenchyma facilitate thermal ablation, including heat insulation and low electrical conductivity. These characteristics permit a larger volume of tissue to be ablated for a given energy than in other tissues in the body [16]. With RFA, an electrode from a generator causes frictional heating, elevating tissue temperature to $6 \mathrm{o}-100^{\circ} \mathrm{C}$. This creates a necrotic zone covering both the tumor and margin of normal parenchyma [17]. However, this thermal energy can be limited by the heat-sink effect of adjacent blood vessels and airways [1]. Several groups have reported that the presence of vessels or bronchi greater than $3 \mathrm{~mm}$ in diameter within the ablation zone is predictors of incomplete local treatment $[18,19]$. Pathologic analyses of 354 NSCLC cases (adenocarcinoma and squamous cell carcinoma) have revealed that 8 and $6 \mathrm{~mm}$ margins, respectively, are needed to cover 95\% of microscopic disease extension [20]. Thus to obtain adequate margins, the ablation zone must exceed the tumor size [21]. RFA therefore performs best for lesions smaller than $2 \mathrm{~cm}$, with rates of complete ablation ranging from $78 \%$ to $96 \%$ with a mean follow-up of 1 year $[3,14,18$, $22,23]$. Lower success rates are seen with tumors $2-3 \mathrm{~cm}$ in diameter $[3,18,22,23]$. More specifically, a ratio of RFA-induced ground-glass opacity (GGO) to tumor area of greater than 4 (bidimensional area on axial images) is correlated with a significantly higher rate of complete ablation than a ratio of less than or equal to 4 [3]. 
MWA uses microwaves to cause friction between water molecules, generating hyperthermia [24]. Unlike RFA, during which only one probe is activated at a time, MWA enables simultaneous energy delivery with multiple probes, thus permitting larger ablation zones than RFA. In animal studies, investigators have reported lung ablation zones as large as 54.8 $\pm 8.5 \mathrm{~mm}$ (mean $\pm \mathrm{SD}$ ) [25]. Clinically, Wolf et al. [4] reported MWA results in 50 patients with pulmonary tumors up to $5 \mathrm{~cm}$. The authors reported an overall recurrence rate of $26 \%$ and a tumor size of larger than $3 \mathrm{~cm}$ being predictive of recurrent disease.

Cryoablation uses compressed argon gas to generate subzero temperatures with ice- ball formation. When temperatures are less than $-40^{\circ} \mathrm{C}$, protein denaturation, cell rupture, and ischemia occur [26]. Unlike heat-based ablation, cryoablation does not create GGO intra-procedurally; instead, the ice ball is used to estimate the ablated margin. Hinshaw et al. [27] examined the temperature isotherms and associated ablation zone size seen with cryoablation in an in vivo porcine lung model. They identified the $-20^{\circ} \mathrm{C}$ isotherm as the zone of coagulative necrosis, with a mean ablation zone diameter of $2.4 \pm 0.2 \mathrm{~cm}$ [27]. Most ablation protocols call for three freeze-thaw cycles to achieve tissue necrosis [27, 28].

Irreversible electroporation, which uses electrical pulses to generate high electrical fields $\left(1500 \mathrm{~V} / \mathrm{cm}^{2}\right)$, was developed as a non-thermal ablation technique. This technique causes cellular apoptosis by creating cell membrane pores and resultant cell lysis. However, in the multicenter phase II trial of 23 patients with metastatic disease, Ricke et al. [29] reported local progression in $61 \%$ of treated patients. They postulated that the high local recurrence was likely because of conductivity differences between the tumor tissue and normal lung parenchyma. Given these results, irreversible electroporation requires refinement before becoming an effective ablation modality for pulmonary malignancies.

\section{IMAGING AND RESPONSE}

CT is the preferred image guidance modality for thermal ablation because it provides excellent contrast between the tumor tissue and normal aerated lung. CT provides multiplanar imaging, which facilitates accurate and quick probe placement [30]. In fact, operators who have access to multiplanar imaging have been shown to better distinguish between margin- and lesion-centered probe placement than operators who have access to axial images alone [31]. Cone-beam CT has been used for lung ablation because it facilitates ablation approaches from any angle; however, it lacks real-time imaging capability. This can be an issue in the setting of moving target lesions [32].
CT is also the modality of choice for follow-up after ablation. After ablation, the targeted lesion is replaced by a central area of increased radiographic density that is surrounded by GGO [11], which may overestimate the actual pathologic margins of RFA [33] and cryoablation [28]. An ablation margin surrounding the target lesion of less than 4.5 $\mathrm{mm}$ or an incomplete margin has been correlated with local tumor progression [34]. GGO margins of less than $3 \mathrm{~mm}$ have been associated with tumor progression as well [35]. During the initial post-ablation period ( $<2$ months), this dense opacification and surrounding GGO serve as the new "baseline" post-ablation image [21, 36]. Any increase in the size of this lesion on follow-up should be considered local progression.

The evolution of the imaging appearance of the ablation zone varies depending on the thermal ablation modality. Palussière et al. [37] described the involution pattern seen after RFA of 350 treated lesions with CT follow-up to 12 months. Although they reported five different imaging patterns, they concluded that none of these patterns can be used to exclude local tumor progression. RFA-treated lesions show a relatively slow rate of involution, with a $40 \%$ decrease in size at 15 months after treatment [38]. Similar post-ablation imaging findings have been described after MWA [4] (Figure 1). Cryoablated lesions, however, show faster involution on CT follow-up so that local tumor progression can be visualized as early as 6 months after treatment [39]. CT enhancement patterns after both RFA and cryoablation have not shown correlation with local progression $[6,39]$. CT is therefore primarily used to examine morphologic changes to the ablation zone. However, detecting local progression at follow-up using CT alone remains difficult. Beland et al. [40] found that in 79 patients with NSCLC treated using RFA, local progression was seen in only $1.5 \%$ at 6 months, increasing to $10.1 \%$ at 1 year and $28 \%$ at 2 years. De Baère et al. [13] retrospectively examined the records and images of 566 patients with metastatic disease treated with RFA and reported rates of local tumor progression of $10.4 \%$ at 1 year and $18.1 \%$ at 4 years.

To overcome the limitations of CT's morphologic assessment of local recurrence, PET/CT follow-up has been explored (Table 1). Yoo et al. [41] examined the role of PET/CT in 30 patients with stage I NSCLC treated with RFA. They reported that imaging 6 months after RFA more accurately reflected outcomes at 1 year than imaging performed immediately after RFA [41]. Suzawa et al. [33] studied the utility of PET/CT in the follow-up of 143 patients treated with RFA. Local tumor progression was seen in $20.4 \%$ of tumors with a median follow-up of 24 months, and PET's diagnostic performance exceeded that of CT at all follow-up imaging time points. However, for up to 3 months after ablation, PET/CT can be confounded by inflammatory changes in both the treated tissues and mediastinum [40]. Split-dose PET/CT has been developed to aid in target localization and evaluation of treatment effectiveness intraprocedurally $[42,43]$ (Figures 2-3) 

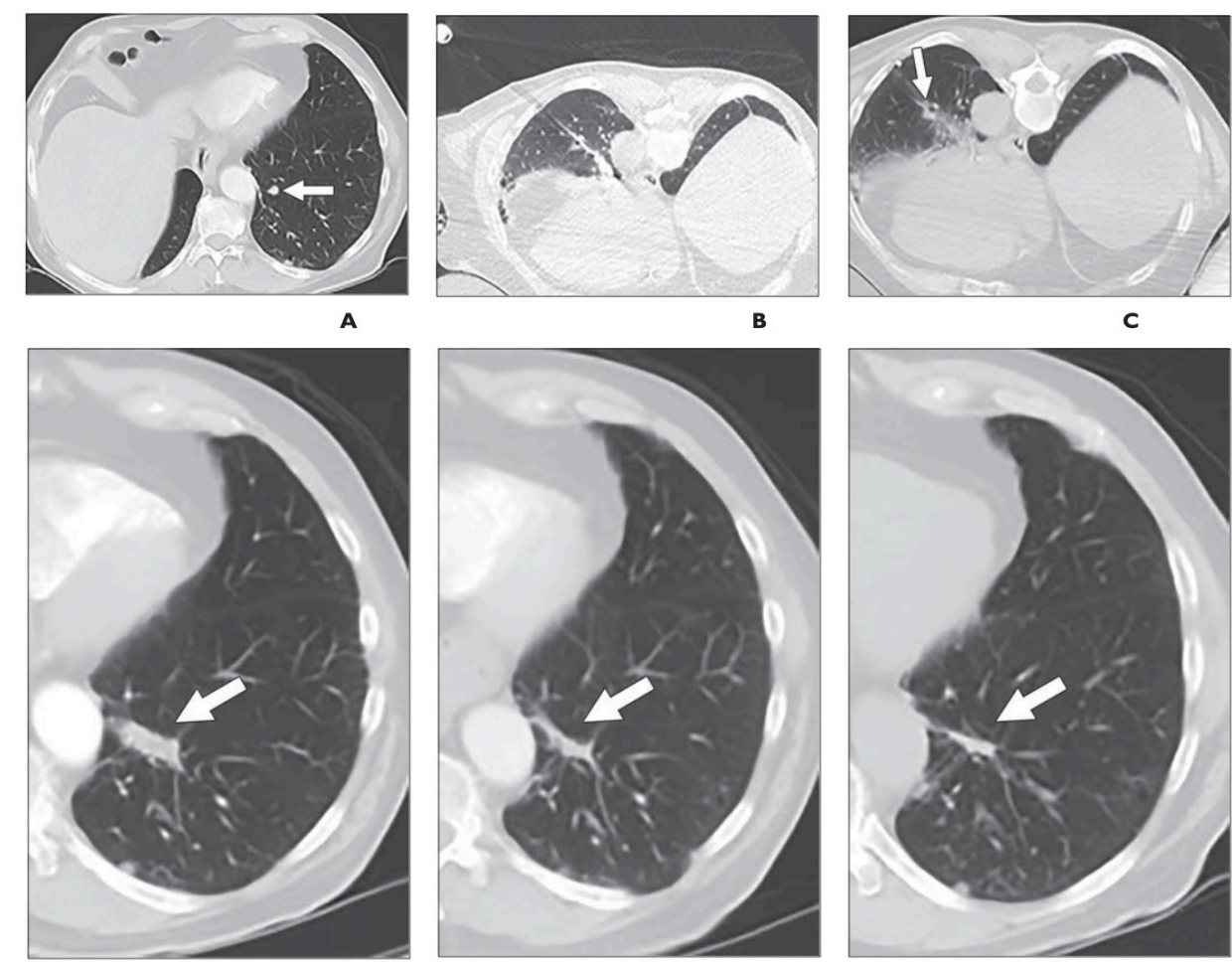

D

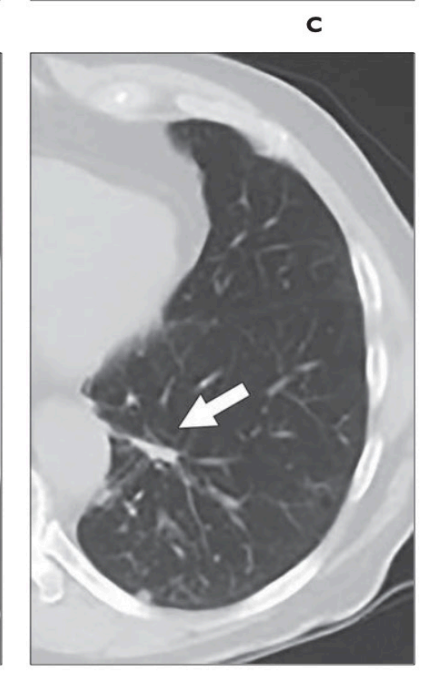

Figure 1 Successful microwave ablation (MWA) of recurrent non-small cell lung carcinoma (NSCLC) metastasis in the left lower lobel with gradual constriction of ablation zone

This case illustrated value of locoregional therapy in general and ablation specifically in treatment of primary lung cancer with recurrent metastatic nodules. Over course of treatment, patient had minimal and stable pulmonary symptoms. In this 72-year old man, initial diagnosis in 2008 was right upper lobe lung adenocarcinoma, stage IB. Patient underwent right upper lobectomy and right lower lobe wedge resection with tumor-free margins for moderately differentiated adenocarcinoma with micropapillary (60\%), papillary (30\%) and lepidic (10\%) components. Patient received no adjuvant chemotherapy and there was no evidence of disease for 20 months. In 2010, imaging detected new clustering of right lower lobe nodules, which were biopsy-proven adenocarcinoma and were morphologically similar to primary tumor. There was no evidence of disease elsewhere. Because of undetermined staging of the disease (metastatic vs second primary tumor), no systematic therapy was used and patient was treated with image-guided radiation therapy. In 2012, imaging showed left lung nodule, and patient was diagnosed with stage IV disease treated with MWA. After MWA, new left upper lobe nodule was treated with wedge resection in January 2013. Subsequent recurrences in two right lower lobe metastases in February 2013 and right lower lobe in May 2014 were treated with MWA. Patient's disease was stable until December 2015 when chest CT revealed increasing left lower lobe nodule, recurrent tumor 3 years after MWA, but not in

area of prior MWA.

A, CT scan obtained before MWA shows $0.9 \times 0.9 \mathrm{~cm}$ left lower lobe lung metastasis (arrow)

B, CT scan obtained during MWA. Metastasis was treated in three overlapping ablations.

C, CT scan obtained immediately after MWA shows ablation defect (arrow) that is $2.5 \times 1.8 \mathrm{~cm}$. Margins were assessed as $5 \mathrm{~mm}$ and extend to aorta.

D-F, Follow-up CT scans obtained 11 weeks (D), 7 months (E), and 1 year (F) after MWA show expected gradual constriction of ablation zone (arrows) and no evidence of local recurrence. Patient remained without disease until February 2017 when he developed bilateral lung nodules with associated rib destruction and increased pleural thickening consistent with multifocal progression of disease. Patient is currently undergoing systemic therapy for disease management.

Table 1 Modification of the Response Evaluation Criteria in Solid Tumours (RECIST) as presented by Herrera et al. [96 ${ }^{\mathrm{a}}$

\begin{tabular}{|c|c|c|c|}
\hline Response & CT Mass Size (RECIST) & CT Mass Quality & PET $^{\mathrm{b}}$ \\
\hline $\begin{array}{l}\text { Complete ( } 2 \text { of the } \\
\text { criteria) }\end{array}$ & $\begin{array}{l}\text { Lesion disappearance (scar) or } \\
<25 \% \text { of original size }\end{array}$ & $\begin{array}{l}\text { Cyst cavity formation, low } \\
\text { density }\end{array}$ & SUV $<2.5$ \\
\hline Partial ( 1 of the criteria) & $\begin{array}{l}>30 \% \text { Decrease in sum LD of } \\
\text { target lesions }\end{array}$ & $\begin{array}{l}\text { Mass central necrosis or } \\
\text { central cavity with liquid } \\
\text { density }\end{array}$ & $\begin{array}{l}\text { Decreased SUV or area of } \\
\text { FDG uptake }\end{array}$ \\
\hline $\begin{array}{l}\text { Stable lesion ( } 1 \text { of the } \\
\text { criteria) }\end{array}$ & $\begin{array}{l}<30 \% \text { Decrease in sum LD of } \\
\text { target lesions }\end{array}$ & $\begin{array}{l}\text { Mass solid appearance, no } \\
\text { central necrosis or cavity }\end{array}$ & $\begin{array}{l}\text { Unchanged SUV or area } \\
\text { of FDG uptake }\end{array}$ \\
\hline $\begin{array}{l}\text { Progression (2 of the } \\
\text { criteria) }\end{array}$ & $\begin{array}{l}\text { Increase }>20 \% \text { in sum LD of } \\
\text { target lesions }\end{array}$ & $\begin{array}{l}\text { Solid mass, invasion adjacent } \\
\text { structures }\end{array}$ & $\begin{array}{l}\text { Higher SUV or larger area } \\
\text { of FDG uptake }\end{array}$ \\
\hline
\end{tabular}

all target lesions LI, Fernando HC, Perry Y, et al. Radiofrequency ablation of pulmonary malignant tumours in nonsurgical candidates, Copyright 2003) $[96$

政 Tumours treated with radiofrequency ablation 

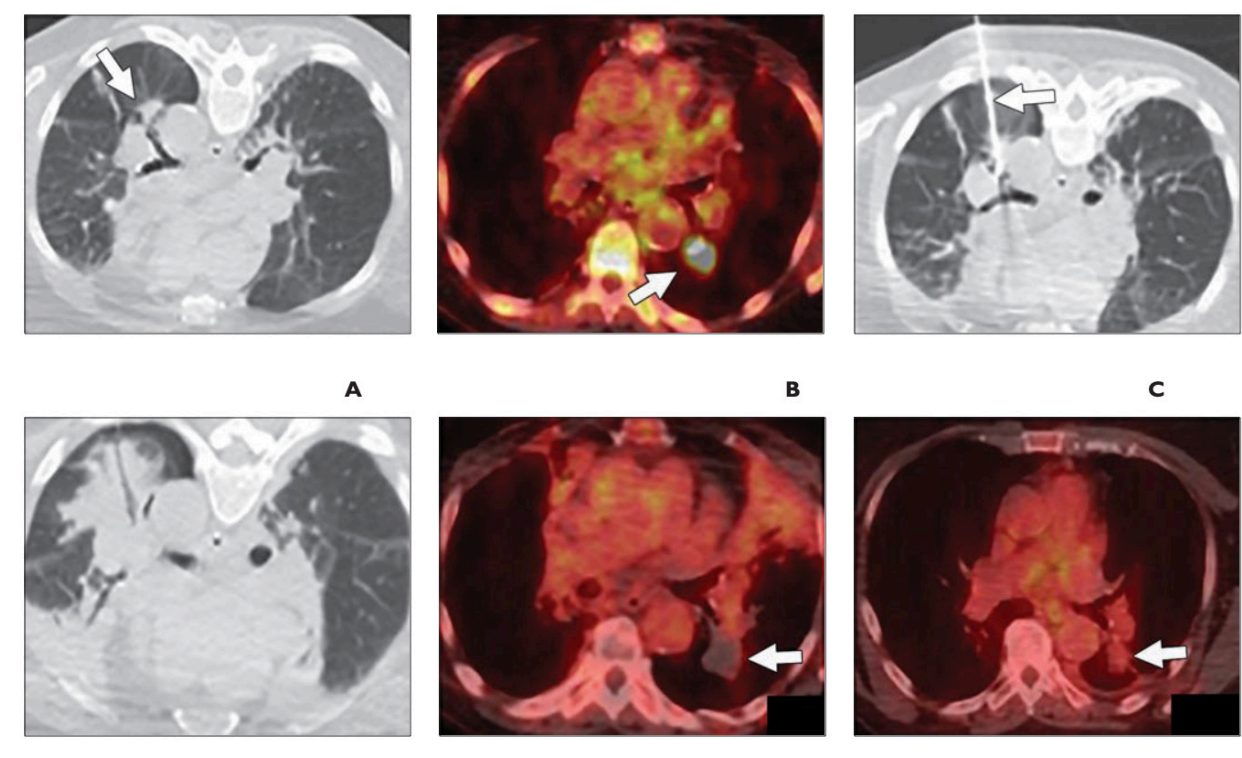

D

E
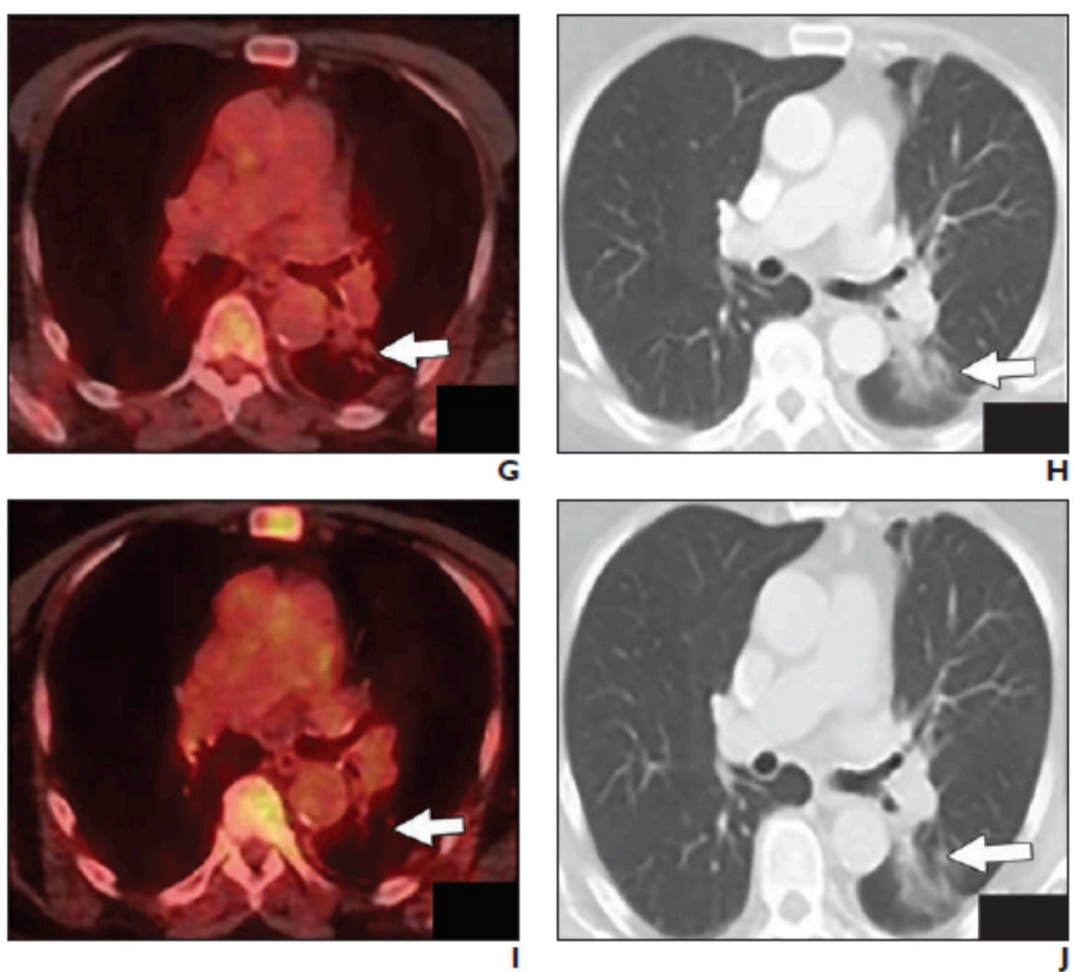

Figure 2 Successful microwave ablation (MWA) of recurrent biopsy-proven non-small cell lung carcinoma (NSCLC) in 83-year-old woman
Initial diagnosis in May 2007 was stage IA bronchoalveolar adenocarcinoma in right upper lobe. Wedge resection of left upper and lower lobes was performed, and malignancy was pathologically proven only in left upper lobe. Left lower lobe FDG-avid nodule remained in situ after resection. This nodule had interval enlargement and was found to be positive for adenocarcinoma in November 2007 at biopsy. This nodule was treated with radiofrequency ablation in March 2008, with no viable Ki-67-positive tumor cells detected on tissue adherent to electrodes [90]. In November 2011 patient returned with new biopsyproven NSCLC nodule in left lower lobe. This nodule was treated with MWA

A, Cross-sectional CT scan shows 2,8 cm recurrent NSCLC lesion (arrow) in left lower lobe.

B, Pre-treatment split-dose ${ }^{18} \mathrm{~F}-\mathrm{FDG}$ PET/CT scan shows FDG-avid lesion (arrow) with maximum standardized uptake value of 5.9. Split-dose ${ }^{18}$ FDG PET/CT is technique for PET/CT-guided ablation that permits both target localization and evaluation of treatment effectiveness. During procedure, standard administered diagnostic FDG activity dose of approximately $12 \mathrm{mCi}$ [ $444 \mathrm{MBq}$ ] is administered in two aliquots: 4-mCi [148 MBq] target dose or imaging dose administered 30-6o minutes before ablation and 8-mCi [296 MBq] treatment efficacy dose administered immediately after ablation. Images are obtained 30 minutes later

C, CT scan obtained during MWA shows microwave electrode (arrow) in tumor: two overlapping ablations of 8 minutes each were performed at 10-25 W.

D, CT scan obtained immediately after MWA shows ablation zone. All margins except medial margin are more than $5 \mathrm{~mm}$. Smaller medial margin extends to aorta.

E, Split dose ${ }^{18}$ FDG PET/CT scan obtained immediately after MWA shows no residual FDG avidity in the area (arrow).

F, ${ }^{18} \mathrm{FDG}$ PET/CT scan obtained 6 months after MWA shows expected constriction of ablated zone (arrow) and no FDG avidity.

G and $\mathbf{H}$, FDG PET/CT scan (G) and corresponding CT scan (H) obtained 1 year after MWA show further constriction of ablation zone (arrows).

I and J, FDG PET/CT scan (I) and corresponding CT scan (I) obtained 2 years after MWA show expected evolution of ablation zone (arrows) with no evidence of local recurrence. 

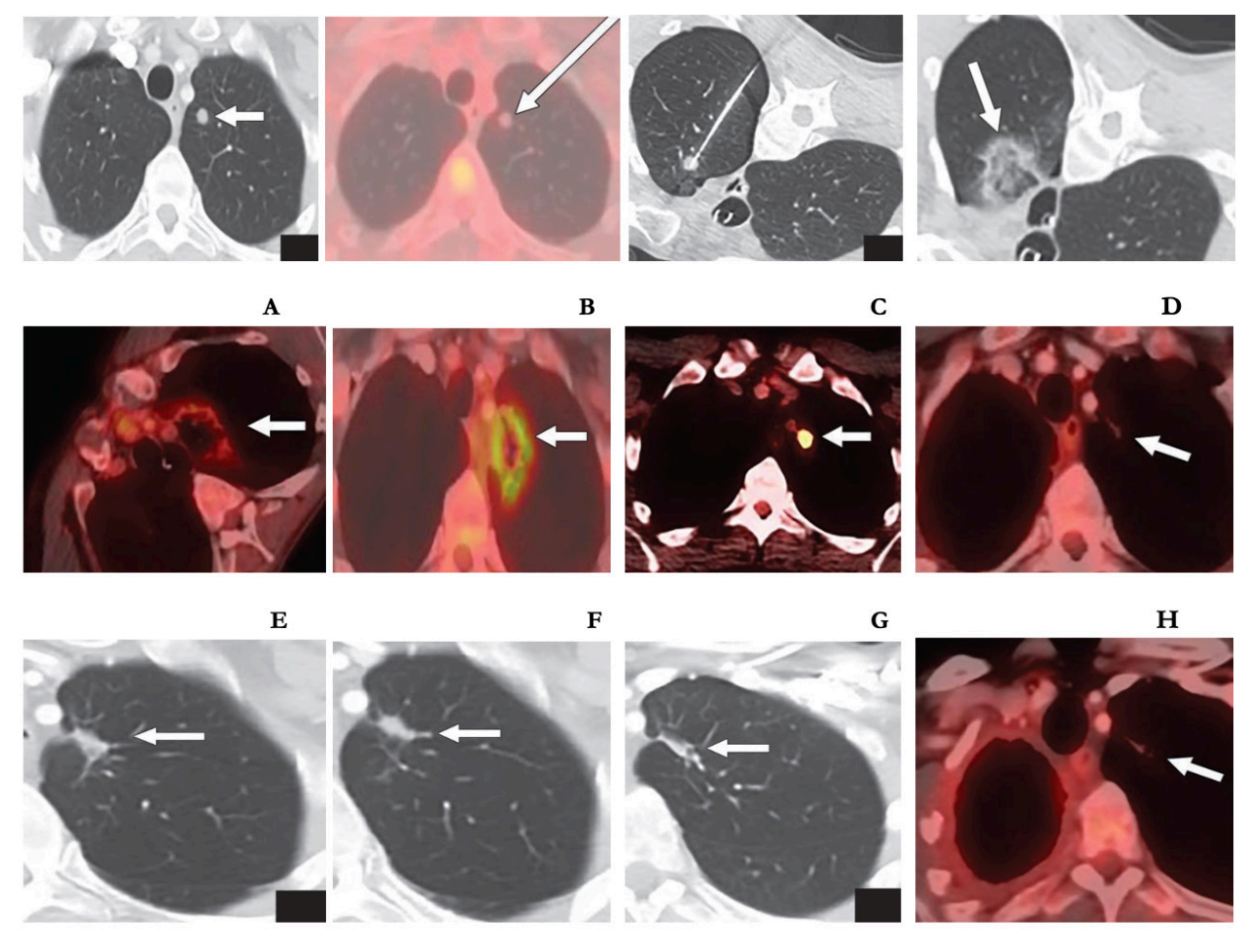

$\mathbf{K}$

Figure 3-Successful microwave ablation (MWA) of lung metastases from colorectal carcinoma in 37-year-old man

The patient had a history of ulcerative colitis and underwent proctocolectomy and formation of J-pouch in 1996. In June 2010, patient was diagnosed with stage I mucinous adenocarcinoma of rectum and received adjuvant chemotherapy (folic acid, 5 -fluorouracil, oxaliplatin) and chemoradiotherapy. In April 2012, patient developed right middle lobe lung metastasis and underwent right middle lobe wedge resection. In February 2013 when patient was no longer receiving chemotherapy, follow-up imaging showed significant progression of disease in left lung with two growing nodules in left lower lobe and left upper lobe. Both lesions underwent MWA with at least 5-mm ablation margins. Tissue from ablation electrodes showed no viable tumor cells [90]. We present imaging of one of two ablated lesions.

A, Pretreatment cross-sectional CT scan shows $8 \times 10 \mathrm{~mm}$ medial left upper lobe lung metastasis (arrow). B, Fluorine-18-FDG PET/CT scan shows mildly FDG-avid lesion (arrow).

C, CT scan obtained during MWA shows microwave electrode in tumor. Two overlapping ablations, $45 \mathrm{~W}$ for 10 minutes and $65 \mathrm{~W}$ for 5 minutes, were performed.

D, CT scan obtained immediately after ablation shows ablation zone (arrow) of $28 \times 30 \times 30 \mathrm{~mm}$ with minimal ablation margin of more than $10 \mathrm{~mm}$.

E, Split-dose ${ }^{18} \mathrm{~F}$-FDG PET/CT scan obtained after second dose shows no uptake in ablated zone (arrow); maximum standardized uptake value $\left(\mathrm{SUV}_{\max }\right)$ is 1.o. Histopathologic analysis of tissue from ablation

electrodes showed no viable tumor cells [9o]. Split-dose ${ }^{18} \mathrm{FDG}$ PET/CT is technique for PET/CT-guided ablation that permits both target localization and evaluation of treatment effectiveness. During procedure, standard administered diagnostic FDG activity dose of approximately $12 \mathrm{mCi}$ [444 MBq] is administered in two aliquots: 4 -mCi [148-MBq] target dose or imaging dose administered $30-60$ minutes before ablation and 8-mCi [296-MBq] treatment efficacy dose administered immediately after ablation. Images are obtained 30 minutes later.

F, ${ }^{18} \mathrm{FDG}$ PET/CT scan obtained 5 weeks after MWA shows hypermetabolic rim of cavitated ablation zone (arrow). Ablation zone is $2.9 \times 4.1 \mathrm{~cm}$ with $\mathrm{SUV}_{\max }$ of 5.5. Follow-up imaging finding of hypermetabolic rim of cavitated ablation zone was characterized as expected post-ablation change rather than as residual or recurrent disease. Cavitated ablation zone with hypermetabolic rim can erroneously be interpreted as evidence of local tumor progression. Sequential imaging follow-up with standardized uptake value readings is helpful to detect gradual resolution. In cases of focal SUV increase in ablation zone and whenever in doubt, short-interval PET or biopsy is recommended with aim of repeat treatment.

G, ${ }^{18}$ FDG PET/CT scan obtained 4 months after MWA shows typical collapse and constriction of cavitated ablation zone (arrow), which is $1.6 \times 1 \mathrm{~cm}$, and decreased FDG avidity of ablation zone to SUV'max of 4.7 . $\mathbf{H},{ }^{18} \mathrm{FDG}$ PET/CT scan obtained 7 months after MWA shows no FDG uptake in ablated area (arrow). I, CT scan obtained 1 year after MWA shows constricted ablation zone (arrow).

J and $\mathbf{K}, \mathrm{CT}$ scans obtained 2 years $(\mathbf{J})$ and 4 years $(\mathbf{K})$ after MWA show ablation zone (arrows).

L, ${ }^{18} \mathrm{FDG}$ PET/CT scan obtained 4 years after ablation shows no FDG uptake in ablated area (arrow). After ablation of left lung metastases, patient developed right lung metastasis, which was also treated by MWA. In November 2014, patient developed pleural metastases and pleural-based metastases, which were treated with three regimens of systemic chemotherapy. Patient was alive at last follow-up in 2017.

\section{CLINICAL OUTCOMES}

Non-Small Cell Lung Carcinoma

An early study by Simon et al. [44] reported a median overall survival (OS) of 29 months ( $95 \% \mathrm{CI}, 2 \mathrm{O}-38$ months) in 75 patients with NSCLC, with 1- and 5-year survival rates of $78 \%$ and $27 \%$. They found improved survival in patients with lower stage disease and lesions less than $3 \mathrm{~cm}$ in diameter [44]. Ambrogi et al. [45] and Huang et al. [46] reported similar 5 -year survival rates of $25 \%$ in patients with stage I NSCLC. Huang et al. reported a local progression rate of $23.7 \%$, with a significantly increased rate of local progression in patients with lesions larger than $4 \mathrm{~cm}$. More recent studies have reported improved survival, which is likely a combination of multiple factors, including technical refinements, better patient selection, and the use of targeted systemic therapies [30]. Palussière et al. [37] reported a 5 -year OS rate of $58.1 \%$ and disease-free survival rate of $27.9 \%$ in 87 patients with NSCLC after thermal ablation. On multivariate analysis, tumor size larger than $2 \mathrm{~cm}$ was an 
independent prognostic factor for disease-free survival [37]. Dupuy et al. [47] reported the outcomes of the American College of Surgeons Oncology Group trial of 51 patients with stage IA NSCLC tumors less than $3 \mathrm{~cm}$ in diameter. These authors reported 1- and 2-year OS rates of $86.3 \%$ and $69.8 \%$, with 2-year survival rates increasing to $83 \%$ for patients with lesions less than $2 \mathrm{~cm} \mathrm{[47].} \mathrm{Similar} \mathrm{results} \mathrm{were} \mathrm{seen} \mathrm{by} \mathrm{Hiraki} \mathrm{et} \mathrm{al.} \mathrm{[48]} \mathrm{and} \mathrm{Liu} \mathrm{et} \mathrm{al.} \mathrm{[49]} \mathrm{in}$ patients with stage I NSCLC. Both groups reported 1-year OS rates ranging from 90\% to $94 \%$ and 3 -year OS rates ranging from $74 \%$ to $79 \%$. In addition to baseline tumor characteristics, patient comorbidities are predictive of survival. In 82 patients with NSCLC treated with RFA, Simon et al. [50] reported that 40 patients died; however, only 19 patients died due to disease progression. On multivariate Cox regression analyses, the Charlson comorbidity index (CCI), a measure of comorbidities, was strongly predictive of OS. A CCI of greater than 5 (mean OS, 10.43 months) was associated with significantly increased mortality versus a score of 1-2 (mean OS, 55.5months) or 3-4 (mean OS, 36.62 months). No significant difference in survival was seen between CCI scores of 1-2 and 3-4 [50].

Long-term data after MWA for the treatment of NSCLC are limited. Wolf et al. [4] reported outcomes after MWA of NSCLC in 30 patients. With a mean follow-up of 10 months, Wolf et al. found a local recurrence rate of $26 \%$, which was significantly increased in lesions larger than $3 \mathrm{~cm}$ on logistic regression analysis. OS rates ranged from $65 \%$ at 1 year to $45 \%$ at 3 years, which were not associated with tumor size [4]. Lu et al. [51] reported 1- and 3-year OS rates of $75 \%$ and $29.2 \%$ after MWA in 48 patients with NSCLC of various stages (I-IV). A significantly increased risk of local progression was seen for lesions larger than $4 \mathrm{~cm}$. Belfiore et al. [52] reported 1- and 3-year cancer-specific survival (CSS) of $69 \%$ and $49 \%$ after MWA in 44 patients with lung cancer of various disease stages. A formal survival analysis was omitted due to cohort heterogeneity. In 47 patients with stage I NSCLC treated with MWA, Yang et al. [53] reported local control rates of $96 \%$ and $48 \%$ at 1 and 5 years after MWA. Median CSS and OS were 47.4 and 33.8 months, with significantly improved survival in patients with lesions less than $3.5 \mathrm{~cm}$. Zheng et al. [54] studied outcomes in 183 patients ( 138 of whom had primary lung cancer) treated with MWA. The local progression rate was $19.1 \%$, with an increased risk of progression seen in patients with lesions larger than $3 \mathrm{~cm}$ and in the setting of emphysema on multivariate analysis [54]. The authors reported a median progression-free survival (PFS) and CSS of 16.5 and 29.0 months. Tumor stage and diameter greater than $3 \mathrm{~cm}$ were independent risk factors for CSS on multivariate analysis. A recent retrospective study by Healey et al. [55] examined outcomes of 108 patients ( 82 NSCLCs) who underwent MWA with a median follow-up of 14.1 months. The authors found significantly higher rates of technical success with lesions less than $3 \mathrm{~cm}$ in diameter. The median time to tumor recurrence was 62 months, with recurrence rates ranging from $22 \%$ at 1 year to $44 \%$ at 3 years. Significantly lower rates of recurrence were seen with lesions less than $3 \mathrm{~cm}$. The median OS was 27.1 months, with 1- and 3-year OS rates of $78 \%$ and $39 \%$.

Although the long-term studies on cryoablation of NSCLC are limited, the initial studies have yielded promising results. Yamauchi et al. [56] used cryoablation to treat 34 NSCLC stage I tumors and reported a median survival of 68 months and a 2-year OS rate of $88 \%$; these results are comparable to results seen with RFA. Moore et al. [57] reported a 5-year OS rate of $67.8 \%$ and a local recurrence rate of $14.9 \%$ in 45 patients with T1 NSCLC. A recent study by McDevitt et al. [58] of 25 patients with NSCLC found a median OS of 43 months (95\% CI, 30-57 months) with a median PFS of 15 months (95\% CI, 8.7-20 months).

Thermal ablation has a role in the setting of advanced disease or as salvage therapy. Kodama et al. [59] examined RFA of 51 recurrent NSCLCs that were initially treated with surgery and reported a 1-year survival rate of $97.7 \%$ and a 5 -year survival rate of $55.7 \%$. The authors found that tumor size less than $3 \mathrm{~cm}$ predicts prognosis, with 1 - and 3 -year survival rates of $100 \%$ and $79.8 \%$ for tumors greater than $3 \mathrm{~cm}$ versus $83.3 \%$ and $31.3 \%$ for smaller tumors [59]. In patients with stage III or IV NSCLC, Lee et al. [6o] found longer median OS in patients treated with RFA and chemotherapy $(n=24)$ than in those treated with chemotherapy alone ( $n=18$ ) ( 42 vs 29 months, respectively; $p<0.03$ ). Ablation has also been used in patients with a solitary lung after pneumonectomy, a population with few treatment options for new or recurrent disease and limited pulmonary reserve. Hess et al. [12] and Sofocleous et al. [61] have shown acceptable morbidity and safety of ablation in treating these patients. Sofocleous et al. [61] caution that post-ablation pneumonitis may be a concern in these patients and may be mitigated with periprocedural steroid administration.

\section{Metastatic Disease}

Thermal ablation plays a role in the management of metastatic disease in selected patients with limited disease burden that can be ablated with margins. In general, this group includes patients with up to four lesions per lung and with lesions smaller than $3.5 \mathrm{~cm}[6,62-65]$. Given the clinical course of metastatic disease, the therapies should be repeatable and associated with limited effects on pulmonary function $[5,63,66]$. The Radiofrequency Ablation of Pulmonary Tumors Response Evaluation (RAPTURE) trial in 53 patients with metastatic colorectal carcinoma (CRC) reported 1-and 2-year OS rates of $89 \%$ and $66 \%$ [14]. De Baère et al. [13] reported the largest series of pulmonary metastases (566 patients with 1037 metastases) treated with RFA. The authors reported a median OS of 62 months and 1- and 5-year OS rates of $92.4 \%$ and $51.5 \%$ for the entire cohort. On multivariate analysis, the location of the primary tumor, disease-free interval, tumor 
size greater than $2 \mathrm{~cm}$, and presence of more than three metastases were associated with OS. Several studies have reported similar 1- and 3-year OS rates [67, 68]. Most series evaluating RFA for metastatic disease report local progression rates of 10-30\%, especially for lesions larger than $3 \mathrm{~cm}$ [14, 18, 63, 69-71]. Omae et al. [72] reported long- term followup in 123 patients with lung oligometastases treated with RFA. The authors found a 5-year OS rate and recurrence- free survival rate of $62 \%$ and $25 \%$ [72].

MWA has also been explored for the treatment of pulmonary metastatic disease. Vogl et al. [5] reported outcomes of MWA in 80 patients with metastatic disease. The authors found a rate of local recurrence of $26 \%$, with treatment success significantly related to tumor size less than $3 \mathrm{~cm}$ and to peripheral lesions rather than central lesions. The 1- and 2 -year survival rates were $91.3 \%$ and $75 \%$. In a follow-up study in 57 patients with 91 metastatic lesions [73], local progression was seen in $33 \%$ of patients, with a median time to local progression of $22.6 \pm 12.4$ months. On regression analysis, the shape of the primary lesion and energy deployed per unit of tumor volume $\left(<26.7 \mathrm{~J} / \mathrm{mm}^{3}\right)$ were associated with disease progression. In a more recent comparison study, Vogl et al. [74] examined the role of MWA ( $n=47$ patients $)$ and RFA ( $n=41$ patients $)$ in the treatment of CRC metastases. These authors reported significantly improved local control rates with MWA over RFA and no significant difference in OS or PFS [74]. However, these studies did not perform histopathologic analysis of ablation zones, via biopsy or resection, to validate technical success.

Cryoablation has also been shown to be safe and effective in the treatment of pulmonary metastases, although survival data are limited [75]. Yamauchi et al. [76] reported a local progression rate of $26 \%$ with significantly improved PFS with tumors less than $15 \mathrm{~mm}$ in 24 patients with metastatic CRC treated with cryoablation. The 1- and 3-year OS rates were $91 \%$ and $59.6 \%$ and the 1 - and 3 -year PFS rates were 90.8\% and 59\%. McDevitt et al. [58] found a mean time to local progression of 10 months, with a median OS of 22 months across tumor types treated with cryoablation. A tumor diameter of greater than $3 \mathrm{~cm}$ was associated with local progression. Early results from the Evaluating Cryoablation of Metastatic Lung/Pleura Tumors in Patients-Safety and Efficacy (ECLIPSE) trial [77] of 40 patients with 6o lesions are currently being analyzed. The multicenter prospective cohort includes patients with colon (40\%), renal (23\%), and sarcoma (8\%) primaries. The authors report a local control rate at 1 year of $94.2 \%$ and a 1-year OS rate of $97.5 \%$. The investigators concluded that these initial results compare favorably with those of other ablation modalities including RFA and MWA [77]. Taken in conjunction with the results of other studies, these results indicate that thermal ablation performs best in tumors less than $3 \mathrm{~cm}$, similar to findings seen with NSCLC.

\section{COMPARATIVE STUDIES}

There are limited comparative studies of thermal ablation with other treatment modalities, including surgical resection and stereotactic beam RT. Kim et al. [78] compared the outcomes of patients with stage I NSCLC treated with RFA $(n=8)$ versus those treated with surgical resection $(n=14)$. Although the rates of local recurrence were higher in the patients treated with RFA, OS was similar for the two groups [78]. Lee et al. [6o] compared survival in patients with stage I and II NSCLC who were treated with RFA $(n=16)$ versus those who were treated with surgery $(n=13)$. Although the patients in the RFA group were significantly older than those in the surgery group, there was no significant difference in OS between the RFA and surgery groups (28.2 and 33.8 months, respectively; $p=0.43$ ) [6o]. Zemlyak et al. [79] studied 64 patients with biopsy-proven stage I NSCLC unfit for standard resection. The patients underwent sublobar resection $(n=25)$, RFA $(n=12)$, or cryoablation $(n=27)$. No significant difference in 3-year OS (sublobar resection, 87.1\%; RFA, 87.5\%; cryoablation, 77\%) or in 3-year CSS (sublobar resection, 90.6\%; RFA, 97.5\%; cryoablation, 90.2\%) was seen.

Using the database from the National Cancer Institute's Surveillance, Epidemiology, and End Results program, Kwan et al. [80] examined survival in 1897 patients with earlystage NSCLC who underwent surgical resection or ablation. After propensity score matching was performed, OS and CSS were not significantly different. Alexander et al. [81] compared 56 patients with stage I NSCLC treated with RFA and 28 patients with stage I NSCLC treated with sublobar resection. The 1-, 2-, and 3-year survival rates were 100\%, $95 \%$, and $83 \%$, respectively, for the patients who underwent surgery and $91 \%, 73 \%$, and $55 \%$ for those who underwent RFA; there was a significant increase in survival in patients who underwent resection compared with those who underwent RFA [81].

There are limited data comparing ablation with stereotactic beam RT in the treatment of NSCLC. Ochiai et al. [82] compared outcomes after RFA $(n=48)$ and stereotactic beam RT $(n=47)$ in patients with solitary tumors less than $5 \mathrm{~cm}$. The authors reported similar 3-year local control and OS rates between the two modalities: $9.6 \%$ and $86.4 \%$ for RFA and 7.0\% and 79.6\% for stereotactic beam RT, with no significant difference between the treatments. However, less than half the patients who underwent stereotactic beam RT had pathologically proven NSCLC [82]. In a pooled analysis of 31 stereotactic beam RT ( $n=2767$ patients) and 18 RFA ( $n=328$ patients) studies, Bilal et al. [83] reported that stereotactic beam RT showed lower local progression rates than RFA (3.5-14.5\% vs 23.7-43\%). OS rates were similar between the two treatments, but 5-year OS rates favored stereotactic beam RT over RFA (47\% vs 20.1-27\%) [83]. 
In the treatment of patients with metastatic disease, RFA compares favorably with surgical resection with reported 5 -year OS rates of 27-70\% [84-86]. Large studies detailing the outcomes of stereotactic beam RT in patients with metastatic disease are limited. Fode and Hoyer [87] examined 321 patients with oligometastatic disease treated with stereotactic beam RT. The authors found a median OS of 2.4 years ( $95 \%$ CI, 2.3-2.7 years), with 1-and 5-year OS rates of $80 \%$ and $23 \%$. In both primary disease and metastatic disease and unlike surgical resection or stereotactic beam RT, thermal ablation techniques can be repeated with limited changes to pulmonary function [5, 47]. This capability is of particular benefit in the setting of recurrent or residual disease.

\section{FUTURE DIRECTIONS}

Local recurrence remains a limitation of ablative therapy. Several predictors of local recurrence have been reported including tumor size and ablation margin [13, 18, 34]. There has been interest in developing additional biomarkers of disease local recurrence and improved predictors of response. In lung cancer specifically, several oncogenic mutations have been associated with prognosis $[88,89]$. Sofocleous et al. [90] reported the utility of Ki-67 protein as a biomarker for response after RFA in 47 treated tumors, both primary and metastatic. Ki-67-positive tumor cells were an independent marker of local tumor progression and shorter PFS and CSS. Ziv et al. [91] reported that KRAS mutation status of an ablated lesion was a significant predictor of local recurrence that was independent of tumor size or ablation margin in 54 patients with primary adenocarcinoma. Conversely, Wei et al. [92] reported that EGFR-positive status did not adversely affect PFS or OS in 61 patients with stage IIIB or IV NSCLC treated with MWA.

The role of inflammatory cytokines and immune markers for determining prognosis in patients with lung cancer is also being explored. RFA-mediated tumor necrosis can stimulate an immune response [93]. Schneider et al. [93] examined the impact of RFA on the profile of serum inflammatory factors and immune suppressive cells in ablation in 12 patients. In patients with disease recurrence, the investigators found elevated levels of tumor necrosis factor- $\mathrm{a}$, chemokine (CC motif) ligand-2, and chemokine ligand-4 and increased nitric oxide production in circulating myeloid-derived suppressor cells. The authors concluded that these factors may serve as early indicators of incomplete ablation of NSCLC.

\section{CONCLUSION}

Thermal ablation has shown safety and efficacy in the treatment of both primary and secondary lung malignancies in nonsurgical candidates. In NSCLC, after propensity score matching was performed, 2-year OS survival rates match those of surgery and stereotactic beam RT [47, 94]. Ablation also plays a role in metastatic disease, showing safety and efficacy. Across ablation modalities, lesion size is the main determinant of treatment success and survival. Furthermore, local progression remains a limitation for these therapies. Development of new tissue and corresponding surrogate image biomarkers for patient risk stratification and earlier detection of recurrence is needed. Further refinements of ablation technique including the intra-procedural development of tissue confirmation of complete tumor ablation with margins can improve local tumor control and significantly lower recurrence rates. Although ongoing prospective clinical trials comparing the efficacy of thermal ablation, stereotactic beam RT, and surgery are unlikely to mature, they could define the role of these therapies and improve patient selection for each treatment [77, 95]. 


\section{REFERENCES}

1. Dupuy DE, Goldberg SN. Image-guided radiofrequency tumor ablation: challenges and opportunities. Part II. J Vasc Interv Radiol 2001; 12:1135-1148

2. Nishida T, Inoue K, Kawata Y, et al. Percutaneous radiofrequency ablation of lung neoplasms: a minimally invasive strategy for inoperable patients. J Am Coll Surg 2002; 195:426-430

3. de Baère T, Palussière J, Aupérin A, et al. Midterm local efficacy and survival after radiofrequency ablation of lung tumors with minimum follow-up of 1 year: prospective evaluation. Radiology 2006 240:587-596

4. Wolf FJ, Grand DJ, Machan JT, Dipetrillo TA, MayoSmith WW, Dupuy DE. Microwave ablation of lung malignancies: effectiveness, CT findings, and safety in 50 patients. Radiology 2008; 247:871-879

5. Vogl T], Naguib NN, Gruber-Rouh T, Koitka K Lehnert T, Nour-Eldin NE. Microwave ablation therapy: clinical utility in treatment of pulmonary metastases. Radiology 2011; 261:643-651

6. Suh RD, Wallace AB, Sheehan RE, Heinze SB, Goldin JG. Unresectable pulmonary malignancies: CTguided percutaneous radiofrequency ablationpreliminary results. Radiology 2003; 229:821-82.

7. Hamada A, Yamakado K, Nakatsuka A, et al. Radiofrequency ablation for colorectal liver metastases: prognostic factors in non-surgical candidates.Jpn J Radiol 2012; 30:567-574

8. Sofocleous CT, Sideras P, Petre EN, Solomon SB. Ablation for the management of pulmonary malignancies. AJR 2011; 197:[web] W581-W589

9. Jemal A, Murray T, Ward E, et al. Cancer statistics, 2005. CA CancerJ Clin 2005; 55:10-30

10. Edge SB, Compton CC. The American Join Committee on Cancer: the 7 th edition of the AJCC
Cancer Staging Manual and the future of TNM. Ann Surg Oncol 2010; 17:1471-1474

11. Abtin FG, Eradat I, Gutierrez AJ, Lee C, Fishbein MC, Suh RD. Radiofrequency ablation of lung tumors: imaging features of the postablation zone. RadioGraphics 2012; 32:947-969

12. Hess A, Palussière J, Goyers JF, Guth A, Baère A, de Baère T. Pulmonary radiofrequency ablation in patients with a single lung: feasibility, efficacy, and tolerance. Radiology 2011; 258:635-642

13. de Baère T, Auperin A, Deschamps F, et al. Radiofrequency ablation is a valid treatment option for lung metastases: experience in 566 patients with 1037 metastases. Ann Oncol 2015; 26:987-991

14. Lencioni R, Crocetti L, Cioni R, et al. Response to radiofrequency ablation of pulmonary tumours: a prospective, intention-to-treat, multicentre clinical trial (the RAPTURE study). Lancet Oncol 2008; 9:621-628

15. Morgan-parkes JH. Metastases: mechanisms, pathways, and cascades. AJR 1995;164:1075-1082

16. Ihara H, Gobara H, Hiraki T, et al. Radiofrequency ablation of lung tumors using a multitined expandable electrode: impact of the electrode array diameter on local tumor progression. J Vasc Interv Radiol 2016; 27:87-95

17. Alexander ES, Dupuy DE. Lung cancer ablation: technologies and techniques. Semin Intervent Radiol 2013; 30:141-150

18. Gillams AR, Lees WR. Radiofrequency ablation of lung metastases: factors influencing success. Eur Radiol 2008; 18:672-677

19. Hiraki T, Gobara H, Takemoto M, et al. Percutaneous radiofrequency ablation combined with previous bronchial arterial chemoembolization and followed by radiation therapy for pulmonary metastasis from hepatocellular carcinoma. J Vasc Interv Radiol 2006; 17:1189-1193

20. Giraud P, Antoine M, Larrouy A, et al. Evaluation of microscopic tumor extension in non-smallcell lung cancer for three-dimensional conforma radiotherapy planning. Int J Radiat Oncol Biol Phys 2000; 48:1015-1024

21. de Baere T, Tselikas L, Gravel G, Deschamps F Lung ablation: best practice/results/response assessment/role alongside other ablative therapies. Clin Radiol 2017 Feb 16 [Epub ahead of print]

22. Hiraki T, Sakurai I, Tsuda T, et al. Risk factors for local progression after percutaneous radiofrequency ablation of lung tumors. Cancer 2006;107:2873-2880

23. Okuma T, Matsuoka T, Yamamoto A, et al. Determinants of local progression after computed tomography-guided percutaneous radiofrequency ablation for unresectable lung tumors: 9-year experience in a single institution. Cardiovasc Intervent Radiol 2010; 33:787-793

24. Robert Sheu Y, Hong K. Percutaneous lung tumor ablation. Tech Vasc Interv Radiol 2013; 16:239-25

25. Planché $\mathrm{O}$, Teriitehau C, Boudabous S, et al. In vivo evaluation of lung microwave ablation in a porcine tumor mimic model. Cardiovasc Intervent Radiol 2013; 36:221-22

26. Gage AA, Baust J. Mechanisms of tissue injury in cryosurgery. Cryobiology 1998; 37:171-186

27. Hinshaw JL, Lee FT, Laeseke PF, Sampson LA, Brace C. Temperature isotherms during pulmonary cryoablation and their correlation with the zone of ablation. J Vasc Interv Radiol 2010;21:1424-1428

28. Hinshaw JL, Littrup PJ, Durick N, et al. Optimizing the protocol for pulmonary cryoablation: a comparison of a dual- and triple-freeze protocol. Cardiovasc Intervent Radiol 2010; 33:1180-1185

29. Ricke J, Jurgens JH, Deschamps F, et al. Irreversible electroporation (IRE) fails to demonstrate efficacy in a prospective multicenter phase II trial on lung malignancies: the ALICE trial. Cardiovasc Intervent Radiol 2015; 38:401-408

30. de Baere T, Tselikas L, Catena V, Buy X, Deschamps F, Palussière J. Percutaneous thermal ablation of primary lung cancer. Diagn Interv Imaging 2016; 97:1019-1024

31. Antoch G, Kuehl H, Vogt FM, Debatin JF, Stattaus J. Value of CT volume imaging for optimal placement of radiofrequency ablation probes in liver lesions.J Vasc Interv Radiol 2002; 13:1155-1161

32. Cazzato RL, Battistuzzi J-B, Catena V, et al. Conebeam computed tomography (CBCT) versus $\mathrm{CT}$ in lung ablation procedure: which is faster? Cardiovasc Intervent Radiol 2015; 38:1231-1236

33. Suzawa N, Yamakado K, Takao M, Taguchi O, Yamada T, Takeda K. Detection of local tumor progression by ${ }^{18} \mathrm{~F}$-FDG PET/CT following lung radiofrequency ablation: PET versus CT. Clin Nucl Med 2013; 38:e166-

34. Anderson EM, Lees WR, Gillams AR. Early indicators of treatment success after percutaneous radiofrequency of pulmonary tumors. Cardiovasc Intervent Radiol 2009; 32:478-483

35. Lee JM, Jin GY, Goldberg SN, et al. Percutaneous radiofrequency ablation for inoperable non-small cell lung cancer and metastases: preliminary report. Radiology 2004; 230:125-134

36. Ahmed M, Solbiati L, Brace CL, et al.; International Working Group on Image-Guided Tumor Ablation; Interventional Oncology Sans Frontières Expert Panel; Technology Assessment Committee of the Society of Interventional Radiology; Standard of Practice Committee of the Cardiovascular and Interventional Radiological Society of Europe. Image-guided tumor ablation: standardization of terminology and reporting criteria-a 10-year update. J Vasc Interv Radiol 2014; 25:1691-1705 
37. Palussière J, Marcet B, Descat E, et al. Lung tumors treated with percutaneous radiofrequency ablation: computed tomography imaging followup. Cardiovasc Intervent Radiol 2011; 34:989-997

38. Jin GY, Lee JM, Lee YC, Han YM, Lim YS. Primary and secondary lung malignancies treated with percutaneous radiofrequency ablation: evaluation with follow-up helical CT. AJR 2004; 183:1013-1020

39. Ito N, Nakatsuka S, Inoue M, et al. Computed tomographic appearance of lung tumors treated with percutaneous cryoablation. J Vasc Interv Radiol 2012; 23:1043-1052

40. Beland MD, Wasser EJ, Mayo-Smith WW, Dupuy DE. Primary non-small cell lung cancer: review of frequency, location, and time of recurrence after radiofrequency ablation. Radiology 2009; 254:301-

41. Yoo DC, Dupuy DE, Hillman SL, et al. Radiofrequency ablation of medically inoperable stage IA nonsmall cell lung cancer: are early posttreatment PET findings predictive of treatment out- come? AJR 2011; 197:334-340

42. Ryan ER, Sofocleous CT, Schoder H, et al. Split- dose technique for FDG PET/CT-guided percutaneous ablation: a method to facilitate lesion targeting and to provide immediate assessment of treatment effectiveness. Radiology 2013; 268:288-29

43. Singnurkar A, Solomon SB, Gonen M, Larson SM, Schoder H. ${ }^{18}$ F-FDG PET/CT for the prediction and detection of local recurrence after radio- frequency ablation of malignant lung lesions. J Nucl Med 2010; 51:1833-1840

44. Simon CJ, Dupuy DE, DiPetrillo TA, et al. Pulmonary radiofrequency ablation: long-term safety and efficacy in 153 patients. Radiology 2007:243:268-275

45. Ambrogi MC, Fanucchi O, Cioni R, et al. Long-term results of radiofrequency ablation treatment of stage I non-small cell lung cancer: a prospective intention-to-treat study. J Thorac Oncol 2011; 6:20442051

46. Huang L, Han Y, Zhao J, et al. Is radiofrequency thermal ablation a safe and effective procedure in the treatment of pulmonary malignancies? Eur J Cardiothorac Surg 2011; 39:348-351

47. Dupuy DE, Fernando HC, Hillman S, et al. Radiofrequency ablation of stage IA non-small cell lung cancer in medically inoperable patients: results from the American College of Surgeons Oncology Group Z4033 (Alliance) trial. Cancer 2015; 121:34913498

48. Hiraki T, Gobara H, Mimura H, Matsui Y, Toyooka S, Kanazawa S. Percutaneous radiofrequency ablation of clinical stage I non-small cell lung cancer. J Thorac Cardiovasc Surg 2011; 142:24-30

49. Liu B, Liu L, Hu M, Qian K, Li Y. Percutaneous radiofrequency ablation for medically inoperable patients with clinical stage I non-small cell lung cancer. Thorac Cancer 2015; 6:327-333

50. Simon TG, Beland MD, Machan JT, Dipetrillo T, Dupuy DE. Charlson comorbidity index predicts patient outcome, in cases of inoperable non-small cell lung cancer treated with radiofrequency ablation. EurJ Radiol 2012; 81:4167-4172

51. Lu Q, Cao W, Huang L, et al. CT-guided percutaneous microwave ablation of pulmonary malignancies: results in 69 cases. World J Surg Oncol 2012; 10:80

52. Belfiore G, Ronza F, Belfiore MP, et al. Patients' survival in lung malignancies treated by microwave ablation: our experience on 56 patients. Eur J Radiol 2013; 82:177-181

53. Yang $\mathrm{X}, \mathrm{Ye} \mathrm{X}$, Zheng $\mathrm{A}$, et al. Percutaneous microwave ablation of stage I medically inoperable non-small cell lung cancer: clinical evaluation of 47 cases.J Surg Oncol 2014; 110:758-763

54. Zheng A, Ye X, Yang X, Huang G, Gai Y. Local efficacy and survival after microwave ablation of lung tumors: a retrospective study in 183 patients. J Vasc Interv Radiol 2016; 27:1806-1814

55. Healey TT, March BT, Baird G, Dupuy DE Microwave ablation for lung neoplasms: a retrospective analysis of long-term results. J Vasc Interv Radiol 2017; 28:206-211

56. Yamauchi Y, Izumi Y, Hashimoto K, et al. Percutaneous cryoablation for the treatment of medically inoperable stage I non-small cell lung cancer. PLoS One 2012; 7:e33223

57. Moore W, Talati R, Bhattacharji P, Bilfinger T. Fiveyear survival after cryoablation of stage I non-small cell lung cancer in medically inoperable patients. Vasc Interv Radiol 2015; 26:312-319

58. McDevitt JL, Mouli SK, Nemcek AA, Lewandowski RJ, Salem R, Sato KT. Percutaneous cryoablation for the treatment of primary and meta- static lung tumors: identification of risk factors for recurrence and major complications. J Vasc Interv Radiol 2016; 27:1371-1379

59. Kodama H, Yamakado K, Takaki H, et al. Lung radiofrequency ablation for the treatment of unresectable recurrent non-small-cell lung cance after surgical intervention. Cardiovasc Intervent Radiol 2012; 35:563-569

6o. Lee H, jin GY, Han YM, et al. Comparison of survival rate in primary non-small-cell lung cancer among elderly patients treated with radiofrequency ablation, surgery, or chemotherapy. Cardiovasc Intervent Radiol 2012; 35:343-350

61. Sofocleous CT, May B, Petre EN, et al. Pulmonary thermal ablation in patients with prio pneumonectomy. AJR 2011; 196:[web]W6o6-W612

62. King J, Glenn D, Clark W, et al. Percutaneous radiofrequency ablation of pulmonary metastases in patients with colorectal cancer. Br J Surg 2004;91:217-223

63. Steinke K, Glenn D, King J, et al. Percutaneous 72. Omae K, Hiraki T, Gobara H, et al. Long-term imaging-guided radiofrequency ablation in patients with colorectal pulmonary metastases: -year follow-up. Ann Surg Oncol 2004; 11:207-212

64. Dupuy DE, Zagoria RJ, Akerley W, Mayo-Smith WW, Kavanagh PV, Safran H. Percutaneous radiofrequency ablation of malignancies in the lung. AJR 2000; 174:57-59

65. Pastorino U, Buyse M, Friedel G, et al. Long-term results of lung metastasectomy: prognostic analyses based on 5206 cases. J Thorac Cardiovasc Surg 1997; 113:37-49

66. Nour-Eldin NE, Naguib NN, Saeed AS, et al. Risk factors involved in the development of pneumothorax during radiofrequency ablation of lung neoplasms. AJR 2009; 193:[web] $\mathrm{W}_{43}-\mathrm{W}_{4} 8$

67. Fanucchi O, Ambrogi MC, Aprile V, et al. Long-term results of percutaneous radiofrequency ablation of pulmonary metastases: a single institution experience. Interact Cardiovasc Thorac Surg 2016; 23:57-64

68. Matsui Y, Hiraki T, Gobara H, et al. Long-term survival following percutaneous radiofrequency ablation of colorectal lung metastases. J Vasc Interv Radiol 2015; 26:303-310

69. Hiraki T, Mimura H, Gobara H, et al. Repeat radiofrequency ablation for local progression of lung tumors: does it have a role in local tumor control? J Vasc Interv Radiol 2008; 19:706-711

7o. Yan TD, King J, Sjarif A, et al. Treatment failure after percutaneous radiofrequency ablation for nonsurgical candidates with pulmonary metastases from colorectal carcinoma. Ann Surg Oncol 2007; 14:1718-1726

Petre EN, Jia X, Thornton RH, et al. Treatment of pulmonary colorectal metastases by radiofrequency ablation. Clin Colorectal Cancer 2013;12:37-44 
survival after radiofrequency ablation of lung oligometastases from five types of primary lesions: a retrospective evaluation. J Vasc Interv Radiol 2016; 27:1362-1370

73. Vogl T], Worst TS, Naguib NN, Ackermann $\mathrm{H}$ Gruber-Rouh T, Nour-Eldin NE. Factors influencing local tumor control in patients with neoplastic pulmonary nodules treated with microwave ablation: a risk-factor analysis. AJR 2013; 200:665-672

74. Vogl TJ, Eckert R, Naguib NN, Beeres M, GruberRouh T, Nour-Eldin NA. Thermal ablation of colorectal lung metastases: retrospective comparison among laser-induced thermotherapy, radio-frequency ablation, and microwave ablation. AJR 2016; 207:1340-1349

75. Inoue M, Nakatsuka S, Yashiro H, et al. Percutaneous cryoablation of lung tumors: feasibility and safety.J Vasc Interv Radiol 2012; 23:295-302

76. Yamauchi Y, Izumi Y, Kawamura M, et al Percutaneous cryoablation of pulmonary metastases from colorectal cancer. PLoS One 2011; 6:227086

77. de Baere T, Tselikas L, Woodrum D, et al. Evaluating cryoablation of metastatic lung tumors in patients: safety and efficacy-the ECLIPSE Trial, interim analysis at year.J Thorac Oncol 2015;10:1468-1474

78. Kim SR, Han HJ, Park SJ, et al. Comparison between surgery and radiofrequency ablation for stage I non-small cell lung cancer. Eur J Radiol 2012, 81:395-399

79. Zemlyak A, Moore WH, Bilfinger TV. Comparison of survival after sublobar resections and ablative therapies for stage I non-small cell lung cancer. J Am Coll Surg 2010; 211:68-72

8o. Kwan SW, Mortell KE, Talenfeld AD, Brunner MC Thermal ablation matches sublobar resection outcomes in older patients with early-stage non-smal cell lung cancer. J Vasc Interv Radiol 2014; 25:1-9
81. Alexander ES, Machan JT, Ng T, Breen LD, DiPetrillo TA, Dupuy DE. Cost and effectiveness of radiofrequency ablation versus limited surgical resection for stage I non-small-cell lung cancer in elderly patients: is less more? J Vasc Interv Radiol 2013; 24:476-482

82. Ochiai S, Yamakado K, Kodama H, et al. Comparison of therapeutic results from radiofrequency ablation and stereotactic body radiotherapy in solitary lung tumors measuring $5 \mathrm{~cm}$ or smaller. Int J Clin Oncol 2015; 20:499-507

83. Bilal H, Mahmood S, Rajashanker B, Shah R. Is radiofrequency ablation more effective than stereotactic ablative radiotherapy in patients with early stage medically inoperable non-small cell lung cancer? Interact Cardiovasc Thorac Surg 2012; 15:258-265

84. Gonzalez M, Poncet A, Combescure C, Robert J, Ris HB, Gervaz P. Risk factors for survival after lung metastasectomy in colorectal cancer patients: a systematic review and meta-analysis. Ann Surg Oncol 2013; 20:572-579

85. Iida T, Nomori H, Shiba M, et al. Prognostic factors after pulmonary metastasectomy for colorectal cancer and rationale for determining surgical indications: a retrospective analysis. Ann Surg2013: 257:1059-1064

86. Pfannschmidt J, Hoffmann H, Dienemann H. Reported outcome factors for pulmonary resection in metastatic colorectal cancer. J Thorac Oncol 2010; 5(suppl 2):S172-S178

87. Fode MM, Hoyer M. Survival and prognostic factors in 321 patients treated with stereotactic body radiotherapy for oligo-metastases. Radiother Oncol 2015; 114:155-160

88. Sonobe M, Kobayashi M, Ishikawa M, et al. Impact of KRAS and EGFR gene mutations on recurrence and survival in patients with surgically resected lung adenocarcinomas. Ann Surg Oncol 2012; 19:347-354

89. Mak RH, Hermann G, Lewis JH, et al. Outcomes by tumor histology and KRAS mutation status after lung stereotactic body radiation therapy for earlystage non-small-cell lung cancer. Clin Lung Cancer 2015; 16:24-32

9o. Sofocleous CT, Garg SK, Cohen P, et al. Ki 67 is an independent predictive biomarker of cancer specific and local recurrence-free survival after lung tumor ablation. Ann Surg Oncol 2013; 20 (suppl 3):S676-S683

91. Ziv E, Erinjeri JP, Yarmohammadi H, et al. Lung adenocarcinoma: predictive value of KRAS mutation status in assessing local recurrence in patients undergoing image-guided ablation. Radiology 2017:282:251-258

92. Wei Z, Ye X, Yang X, et al. Advanced non-small cel lung cancer: response to microwave ablation and EGFR status. Eur Radiol 2017; 27:1685-1694

93. Schneider T, Sevko A, Heussel CP, et al. Serum inflammatory factors and circulating immunosuppressive cells are predictive markers for efficacy of radiofrequency ablation in nonsmall-cell lung cancer. Clin Exp Immunol 2015; 180:467-474

94. Petre EN, Solomon SB, Sofocleous CT. The role of percutaneous image-guided ablation for lung tumors. Radiol Med (Torino) 2014; 119:541-548

95. Crabtree T, Puri V, Timmerman R, et al. Treatment of stage I lung cancer in high-risk and inoperable patients: comparison of prospective clinical trials using stereotactic body radiotherapy (RTOG 0236), sublobar resection (ACOSOG Z4032), and radiofrequency ablation (ACOSOG Z4033).J Thorac Cardiovasc Surg 2013; 145:692-699

96. Herrera LJ, Fernando HC, Perry Y, et al. Radiofrequency ablation of pulmonary malignant tumors in nonsurgical candidates. $J$ Thorac
Cardiovasc Surg 2003; 125:929-937

97. Padhani AR, Ollivier L. The RECIST (Response Evaluation Criteria in Solid Tumors) criteria: implications for diagnostic radiologists. BrJ Radiol 2001; 74:983-986

98. Tsuchida Y, Therasse P. Response evaluation criteria in solid tumors (RECIST): new guidelines. Med Pediatr Oncol 2001; 37:1-3 
Microwave Ablation in the Management of Colorectal Cancer Pulmonary Metastases

I.Kurilova*, A. Gonzalez-Aguirre* , R. G. Beets-Tan, J. Erinjeri, E. N. Petre, M. Gonen, M. Bains, N. E. Kemeny, S. B. Solomon, C. T. Sofocleous

*Shared first authors 


\section{ABSTRACT}

Purpose

To review outcomes following microwave ablation (MWA) of colorectal cancer pulmonary metastases and assess predictors of oncologic outcomes.

Methods

Technical success, primary and secondary technique efficacy rates were evaluated for 50 patients with 9o colorectal cancer pulmonary metastases at immediate, 4-8 weeks post-MWA and subsequent follow-up CT and/or ${ }^{18} \mathrm{~F}-\mathrm{FDG}$ PET/CT. Local Tumor Progression (LTP) rate, LTP-free survival (LTPFS), cancer-specific and overall survivals were assessed. Complications were recorded according to SIR classification.

Results

Median follow-up was 25.6 months. Median tumor size was $1 \mathrm{~cm}(0.3-3.2 \mathrm{~cm})$. Technical success, primary and secondary technique efficacy rates were $99 \%$, 90\% and $92 \%$, respectively. LTP rate was $10 \%$. One-, 2 - and 3 -year LTPFS was: $93 \%, 86 \%$ and $86 \%$, respectively with median LTPFS not reached. Median overall survival was 58.6 months, median cancerspecific survival (CSS) was not reached. One-, 2- and 3-year overall and cancer-specific survivals were $94 \%$ and $98 \%, 82 \%$ and $90 \%, 61 \%$ and $70 \%$, respectively. On univariate analysis, minimal ablation margin $(\mathrm{p}<0.001)$ and tumor size $(\mathrm{p}=0.001)$ predicted LTPFS; with no LTP for minimal margin $\geq 5 \mathrm{~mm}$ and/or tumor size $<1 \mathrm{~cm}$. Pleural-based metastases were associated with increased LTP risk $(\mathrm{p}=0.002$, SHR $=7.7)$. Pre-MWA CEA level $>10 \mathrm{ng} / \mathrm{ml}$ $(\mathrm{p}=0.046)$ and $\geq 3$ prior chemotherapy lines predicted decreased CSS $(\mathrm{p}=0.02)$. There was no 9o-day death. Major complications rate was $13 \%$.

\section{Conclusions}

MWA with minimal ablation margin $\geq 5 \mathrm{~mm}$ is essential for local control of colorectal cancer pulmonary metastases. Pleural-based metastases and larger tumor size were associated with higher risk of LTP. CEA level and pre-MWA chemotherapy impacted CSS.

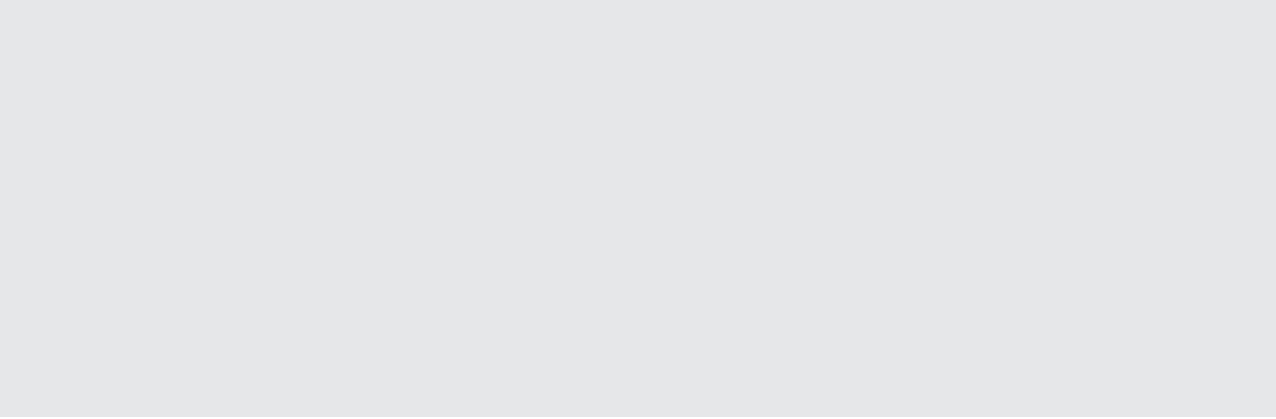

\section{INTRODUCTION}

Approximately $5 \%$ of men and $4 \%$ of women develop colorectal cancer (CRC) during their lifetime, with $20 \%$ of patients having distant metastases at initial diagnosis $[1,2]$. Lung is a second most common metastatic site with incidence of $10 \%-15 \%[3,4]$. Five-year survival of patients with distant disease is roughly $12 \%$ [5]. Complete metastatectomy and/or ablation increase 5 -year survival to $27-68 \%$ [6-9]. However, only minority of patients with CRC pulmonary metastases is surgical candidates due to comorbidities or compromised pulmonary function [10]. In addition, lung metastasectomy recurrences are common (20-68\%) and subsequent surgery is challenging due to limited pulmonary reserve [11-14]. Key ablation advantages compared to metastasectomy include possibility to spare pulmonary parenchyma, lung function and retreatment of new and recurrent metastases, which is often required in pulmonary metastases management [15-25]. Ablation is commonly used in the treatment of patients with limited number of relatively small tumors. Preferably $<3$ tumors in each hemithorax with no, limited or at least controlled extrapulmonary disease are generally accepted pulmonary ablation eligibility criteria [26-29].

Microwave ablation (MWA) has at least theoretical potential to overcome known limitations of radiofrequency ablation (RFA) [20, 21, 25-28, 30, 31], such as the diminished thermal conductivity of aerated lung and the "heat sink phenomenon" (due to flow in nearby vessels or the airway) that can impact the ability to create large and more uniform ablation zones with adequate margins in shorter time as demonstrated in animal studies [32-35]. Consequently, higher rates of complete ablation and sustained tumor control could be expected when using MWA [33-35].

This study assessed oncologic outcomes and complications of MWA in the management of patients with colorectal pulmonary metastases as well as factors affecting these outcomes.

\section{METHODS}

Study population

IRB waiver of approval was obtained for this retrospective review of our prospectively created and maintained HIPAA registered and compliant lung tumor ablation database. All patients with colorectal pulmonary metastases undergoing image-guided MWA between March 2011 and May 2016 were included in the study. 
MWA inclusion criteria were: limited number of metastases (up to 6) in each hemithorax and relatively small tumor size (up to $3.5 \mathrm{~cm}$ ) with no, limited or at least controlled extrapulmonary disease. Central tumor location was not an exclusion criterion if ablation could be performed safely without risk of damaging adjacent structures. The decision to treat the metastasis with ablation was made after multidisciplinary discussion.

Ablation procedure, imaging follow-up and definitions

Operators' experience, pre-ablation imaging and biopsy timing, image guidance, percutaneous entry route, MWA system and electrode choice, prophylactic antibiotics, anesthesia type, bilateral lung metastases and pneumothorax management, thermal monitoring, immediate post-procedure imaging, minimal ablation margin measurement (Figure 1) and imaging follow-up are described in Table 1. All study definitions are described in Table 2.

All patients with LTP were assessed for repeat thermal ablation eligibility.

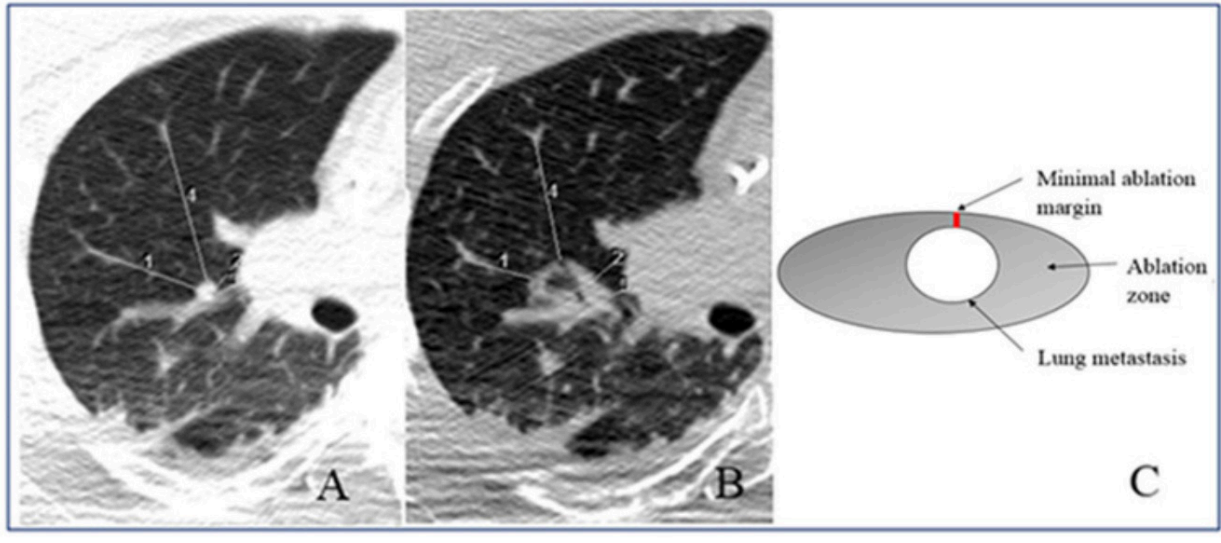

Figure 1 Minimal ablation margin measurement.

A, Pre-ablation measurements: measurement $(1)=34 \mathrm{~mm}$; measurement $(2)=14 \mathrm{~mm}$; measurement $(3)=$ $8 \mathrm{~mm}$; measurement $(4)=50 \mathrm{~mm}$.

B, Post-ablation measurements: measurement $(1)=19 \mathrm{~mm}$; measurement $(2)=12 \mathrm{~mm}$; measurement (3) $=3 \mathrm{~mm} ;$ measurement $(4)=37 \mathrm{~mm}$. After subtracting corresponding measurements at each location (1-4) the respective ablation margins were $15 \mathrm{~mm}, 2 \mathrm{~mm}, 5 \mathrm{~mm}$ and $13 \mathrm{~mm}$. Therefore, minimal ablation margin for this ablation zone was $2 \mathrm{~mm}$ (insufficient).

C, Minimal ablation margin scheme.
Table 1 Ablation procedure descriptions. MWA-microwave ablation

\begin{tabular}{|c|c|}
\hline Parameter & Description \\
\hline Operator's experience & $\begin{array}{l}\text { Majority of tumours (64\%) were treated by two very experienced operators in lung } \\
\text { ablation. Both operators started to perform lung thermal ablation (RFA) since year } \\
\text { 1999, with MWA introduction since the year 20og. Importantly, since the year } 2014 \\
\text { all the lung lesions have been treated solely with MW ablation in our institution, } \\
\text { contributing to increased experience in this technique for all the operators }\end{array}$ \\
\hline Lesion biopsy timing & $\begin{array}{l}\text { It was chosen based on the need to know tumor pathology in disease management } \\
\text { discussions (such as in patients with history of smoking second primary tumor was a } \\
\text { reasonable possibility), as well as based on technical factors (such as the feasibility to } \\
\text { biopsy the tumor without significant risk for bleeding that could obscure the target for } \\
\text { MW ablation) }\end{array}$ \\
\hline Pre-procedure imaging & $\begin{array}{l}\text { All patients underwent pre-ablation cross-sectional CT and/or }{ }^{8} \mathrm{~F}-\mathrm{FDG} \text { PET/CT for tumo } \\
\text { assessment, restaging and pre-procedural planning }\end{array}$ \\
\hline $\begin{array}{l}\text { Intra-procedural image } \\
\text { guidance }\end{array}$ & $\begin{array}{l}\text { Included conventional CT in all patients, with additional CT fluoroscopy and/or split- } \\
\text { dose e }{ }^{8 \mathrm{~F}-\mathrm{FDG}} \mathrm{PET} / \mathrm{CT}[39] \text { at operator's discretion }\end{array}$ \\
\hline Percutaneous entry route & Chosen based on tumor size, location and proximity to adjacent structures \\
\hline $\begin{array}{l}\text { MW system/ electrode } \\
\text { number }\end{array}$ & $\begin{array}{l}\text { Based on operator's preference. Ablation protocol was completed according to each } \\
\text { manufacturer recommendations for the desired ablation size }\end{array}$ \\
\hline Prophylactic antibiotics & 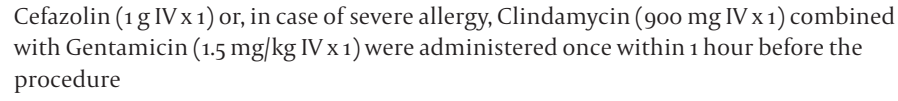 \\
\hline Anaesthesia type & All MWAs were performed under general anaesthesia \\
\hline Number of ablation sessions & $\begin{array}{l}\text { In case of single lung involvement, MWA was completed in a single session. In bilateral } \\
\text { tumours, contralateral lung MWA was performed at least } 3 \text { weeks later to allow recover } \\
\text { of the initially treated lung. In cases of persistent symptoms/persistent pneumothorax } \\
\text { contralateral ablation was delayed until symptoms resolution }\end{array}$ \\
\hline Thermal monitoring & $\begin{array}{l}\text { Used with thermometer tip positioned at: }(1) \text { desired ablation margin; }(2) \text { at structure } \\
\text { that needed be protected from the heat (chest wall, pleural surface); and (3) in the } \\
\text { ablation zone to confirm lethal temperatures }\end{array}$ \\
\hline $\begin{array}{l}\text { Immediate post-procedure } \\
\text { imaging }\end{array}$ & $\begin{array}{l}\text { Non-contrast-enhanced CT scan was obtained to depict the ablation zone as an area of } \\
\text { ground glass opacity covering the target tumor with a minimal ablation margin of at } \\
\text { least } 5 \mathrm{~mm} \text { uniformly around target metastasis }\end{array}$ \\
\hline $\begin{array}{l}\text { Minimal ablation margin } \\
\text { (MM) measurement }\end{array}$ & $\begin{array}{l}\text { It was measured on immediate post-procedure non-contrast-enhanced CT (Figure 1). } \\
\text { MM was defined as the shortest distance between the ablated metastasis and the edge } \\
\text { of the ablation zone. In confluent ablation zones and cases of bleeding obscuring the } \\
\text { ablation zone the MM was not measured }\end{array}$ \\
\hline $\begin{array}{l}\text { Detection and management } \\
\text { of pneumothorax }\end{array}$ & $\begin{array}{l}\text { After ablation, chest X-rays were obtained for at least } 2 \text { hours to detect pneumothorax } \\
\text { prior to patients' release from the post-anaesthesia care unit. Asymptomatic patients } \\
\text { without pneumothorax and with no intravenous analgesia requirement were } \\
\text { discharged home on the same day. Patients with small asymptomatic pneumothoraces } \\
\text { were admitted for overnight observation. Patients with larger, enlarging and/or } \\
\text { symptomatic pneumothoraces were treated with thoracostomy and admitted to the } \\
\text { hospital }\end{array}$ \\
\hline Imaging follow-up & $\begin{array}{l}\text { First follow-up CT and }{ }^{\text {iF}} \text { F-FDG PET/CT were performed at } 4-8 \text { weeks and served as the } \\
\text { new baseline for future evaluations. Subsequent imaging follow-up was performed } \\
\text { approximately every } 3 \text { months thereafter }\end{array}$ \\
\hline
\end{tabular}


Table 2 Study definitions

\begin{tabular}{|c|c|}
\hline Term & Definition \\
\hline Technical success rate & $\begin{array}{l}\text { Percentage of target tumours, successfully treated per ablation protocol, resulting } \\
\text { in the creation of an ablation zone covering the target tumor, depicted on the } \\
\text { immediate post-ablation imaging and on first follow-up imaging (at } 4-8 \text { weeks } \\
\text { post-MWA), which served as the new baseline for future comparisons }\end{array}$ \\
\hline Residual tumor & $\begin{array}{l}\text { Enlargement of the ablation zone or nodular enhancement/ increased focal FDG } \\
\text { uptake ( }\left(\mathrm{VUV}_{\max } \geq 2.5 \text { ) at or within } 1 \mathrm{~cm} \text { from the ablation zone on first post MWA }\right. \\
\text { imaging (at } 4-8 \text { weeks) }\end{array}$ \\
\hline Local tumor progression & $\begin{array}{l}\text { Enlargement of the ablation zone or nodular enhancement/ increased focal FDG } \\
\text { uptake (SUV }{ }_{\text {max }} \geq 2.5 \text { ) at or within } 1 \mathrm{~cm} \text { from the ablation zone. LTP was defined at } \\
\text { the earliest on the second imaging (at 9-16 weeks) post-MWA for those tumours } \\
\text { successfully ablated as depicted at the first ( } 4-8 \text { weeks) post MWA imaging }\end{array}$ \\
\hline Primary technique efficacy rate & $\begin{array}{l}\text { Percentage of target tumours successfully eradicated following the initial MWA } \\
\text { during the entire study follow-up period [40] }\end{array}$ \\
\hline LTP-free survival (LTPFS) & $\begin{array}{l}\text { Time period, calculated from the ablation date until LTP or until the last available } \\
\text { CT and/or }{ }^{\circ} \mathrm{F}-\mathrm{FDG} \text { PET/CT }\end{array}$ \\
\hline Secondary technique efficacy rate & $\begin{array}{l}\text { Percentage of target tumours successfully eradicated following repeat ablation(s) } \\
\text { to treat residual disease or LTP }\end{array}$ \\
\hline Assisted LTPFS & $\begin{array}{l}\text { Cumulative time interval from the initial MWA until the latest follow-up, } \\
\text { including all ablations to treat the initial tumor and all subsequent } \operatorname{LTP}(\mathrm{s}) \text {. }\end{array}$ \\
\hline Side effects & $\begin{array}{l}\text { Side effects included expected, undesired consequences of MWA that did not lead } \\
\text { to unexpected increase of level of care }\end{array}$ \\
\hline Minor complications & $\begin{array}{l}\text { Minor complications included events that did not require intervention or } \\
\text { required only nominal therapy }\end{array}$ \\
\hline Minor complications & $\begin{array}{l}\text { Major complications required intervention, resulted in prolonged hospitalization } \\
\text { ( }>48 \text { hours) or had permanent sequelae }\end{array}$ \\
\hline
\end{tabular}

MWA-microwave ablation, LTP-local tumor progression

\section{Study objectives}

Primary objectives included technical success, primary and secondary technique efficacy rates, LTP rate, LTP-free survival (LTPFS), assisted LTPFS, overall survival (OS) and cancer- specific survival as well as identification of factors, associated with LTPFS and cancer-specific survival.

Secondary study objectives included assessment of side effects and complication according to SIR classification $[27,39]$.

\section{Factors affecting oncologic outcomes}

Tumor-related (tumor size and location) and procedure-related factors (minimal ablation margin) were analyzed as potential predictors of LTPFS.

Patient-related factors (lungs as the first metastatic disease site) and prior therapies (prior lung or liver surgery, prior chemotherapy \pm target therapy lines) were analyzed as potential predictors of cancer-specific survival.
Statistical analysis

Statistical analysis methodology is described in Table 3

Table 3 Statistical analysis methods used in the study

Kaplan Meier metho

Compete risk model, adjusted for

lustering (tumor-based)

\section{Used to evaluate LTPFS, overall and cancer-specific survivals}

Analysed LTPFS predictors (tumor size, minimal margin size and location) ther for multiple patients that underwent ablation for more thon one

Fisher exact test Dert was considered a competing event in this analysis

Othe Used to analyse categorical variables

Software used ample proportion confidence intervals were calculated using Wilson method

Significance level $\mathrm{P}<0.05$ was considered significant

\section{RESULTS}

Patient Population

Fifty patients with go colorectal lung metastases were treated in 6o MWA sessions. Twenty-eight (56\%) of patients were women and 22 (44\%) were men with a mean age of $58.5 \pm 13.2$ years. Median follow-up for survivors was 25.6 months (range: 12.8-80.6 months), 29/50 (58\%) of patients were followed for more than two years (Figures 2-3). At the time of initial diagnosis $21 / 50(42 \%)$ of the patients had AJCC stage IV disease. Thirteen out of fifty (26\%) of patients underwent MWA for disease progression after lung metastasectomy. Patient and tumor characteristics, biopsy timing as well as pre-MWA therapies are described in Tables 4-5.

Technical Parameters and Technical Success Rate

Six out of fifty (12\%) of patients received MWA in two separate sessions to treat bilateral lung metastases, with one patient requiring contralateral ablation delay for a week to allow persistent asymptomatic pneumothorax resolution. Ablation technical parameters are depicted in Table 6.

Technical success rate was 89/90 (99\%): 1/90 (1\%) of ablated tumors (3.2 cm in size and pleural-based) maintained focal metabolic uptake on first post-MWA ${ }^{18} \mathrm{~F}-\mathrm{FDG}$ PET/CT scan, consistent with residual tumor. 


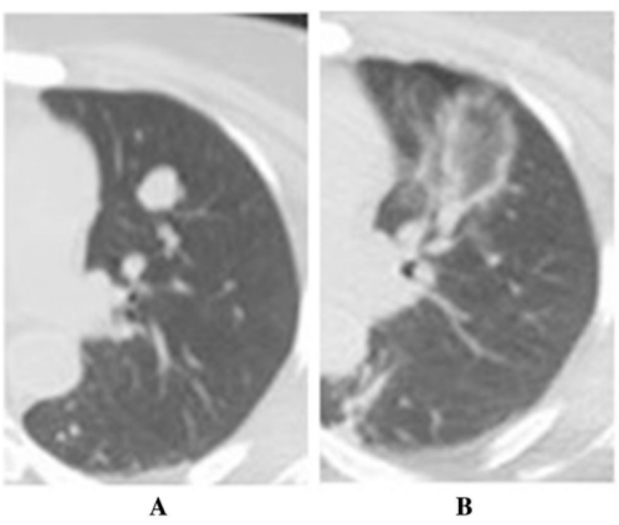

B
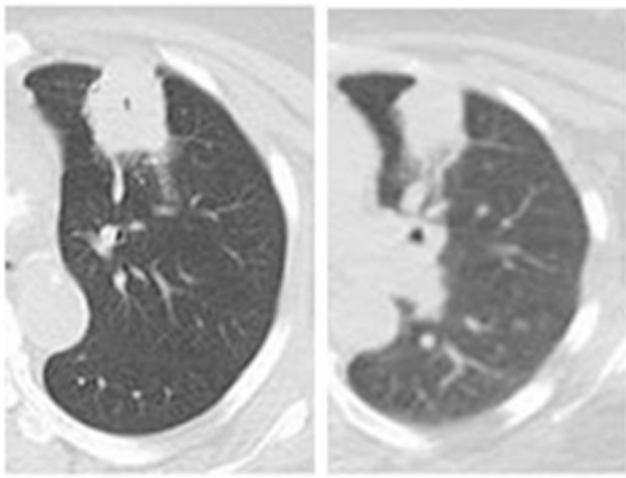

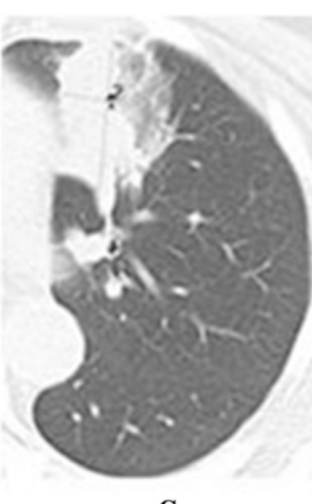

C

$\mathbf{E}$

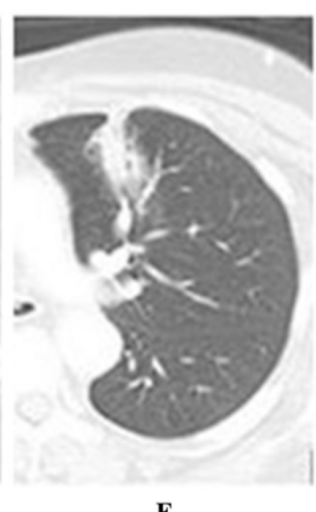

Figure 2 Lung MWA ablation zone changes in 72-year old woman with metastatic colorectal cancer over 28 months follow - up period on cross-sectional CT, showing gradual constriction of ablation zone and no evidence of local tumor progression.

After initial diagnosis, the patient underwent right hemicolectomy, adjuvant chemotherapy with 5 -FU and leucovorin and was off-treatment with no evidence of disease for 18 months.

A, Pre-ablation enlarging lung nodule (from $0.9 \mathrm{~cm}$ to $1.6 \mathrm{~cm}$ ) with rising CEA (from $5.8 \mathrm{ng} / \mathrm{ml}$ to $11.6 \mathrm{ng}$ / $\mathrm{ml}$ ): lesion was considered metastatic, not biopsy-proven. Due to significant comorbidities (end-stage kidney insufficiency, coronary artery disease, diabetes, arterial hypertension and history of stroke) lung ablation was preferred to surgery.

B, Immediate post-MWA CT with ablation zone as ground-glass opacity measuring $3.2 \times 3.0 \mathrm{~cm}$

C, Follow - up scan at 5 weeks, which served as a new baseline for future comparisons.

D, Follow - up scan at 17 weeks.

E, Follow - up scan at 15.5 months;

F, Follow-up scan at 28 months with constricted ablation zone and no evidence of local tumor progression. The patient was off-treatment after MWA with no evidence of disease elsewhere for 2 years, when the patient developed solitary biopsy proven liver metastasis, treated with liver segmentectomy. The patient then was off-treatment with no evidence of disease elsewhere throughout the last follow- up.
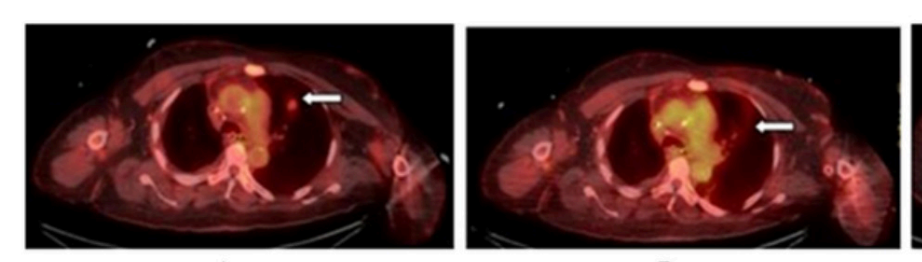

B
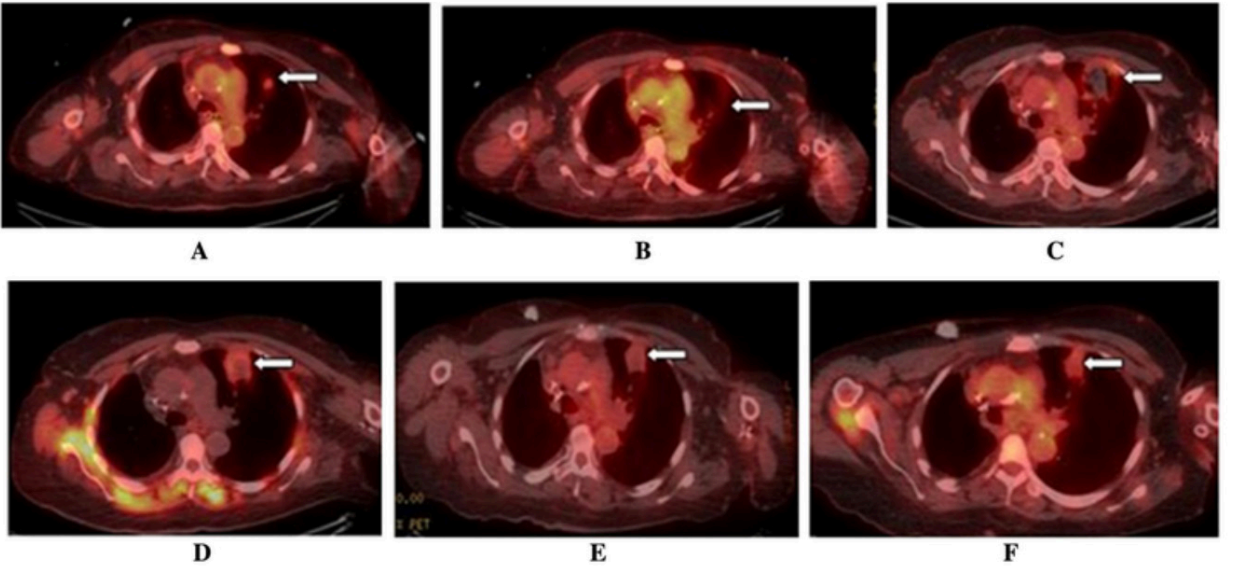

Figure 3 Real time ${ }^{18} \mathrm{~F}-\mathrm{FDG}$ PET/CT - guided lung MWA with ablation zone constriction and decreased metabolic uptake over 28 months follow-up period (imaging of the same patient as in the Figure 1). A, Pre-ablation split-dose ${ }^{8} \mathrm{~F}$-FDG PET/CT scan* with FDG - avid left upper lobe lung metastasis. B, Immediate post-ablation ${ }^{18} \mathrm{~F}$-FDG PET/CT scan with no metabolic uptake in the ablation zone. C, ${ }^{18}$-FDG PET/CT scan 4 weeks after ablation.

D, ${ }^{18}$ F-FDG PET/CT scan 17 weeks post-ablation.

E, ${ }^{8}$ F-FDG PET/CT scan 15.5 months after ablation.

F, ${ }^{18}$ F-FDG PET/CT scan 23 months after ablation with constricted ablation zone, no metabolic uptake and no evidence of local tumor progression.

${ }^{*}$ Split-dose ${ }^{8} \mathrm{~F}$-FDG PET/CT is a technique for PET/CT-guided ablation that permits both target localization and evaluation of treatment effectiveness. During the procedure, the standard administered diagnostic FDG activity dose of approximately $12 \mathrm{mCi}$ ( $444 \mathrm{MBq}$ ) is administered in two aliquots: a 4-mCi (148-MBq) target/imaging dose administered 30-6o minutes before ablation and an 8-mCi (296-MBq) treatment efficacy dose administered immediately after the ablation with images obtained 30 minutes later [43]. 
Table 4 Patient Characteristics

\begin{tabular}{|c|c|}
\hline Parameter & Incidence \\
\hline Number of patients & 50 \\
\hline Number of treated lesions & 90 \\
\hline Number of ablations & 60 \\
\hline Lesion size (median, cm) & $1 \mathrm{~cm}$ (range, $0.3-3.3 \mathrm{~cm}$ ) \\
\hline Time from primary diagnosis to lung metastases (median, in months) & 27.9 (range, 12.1-1411.2) \\
\hline Time from lung metastases diagnosis to MWA (median, in months) & 9.4 (range, 0.4-106.9) \\
\hline Time from lung resection to MWA (median, in months) & $25.5($ range, 1.9-153.2) \\
\hline \multicolumn{2}{|l|}{ Target tumor biopsy } \\
\hline Performed within 2-6 weeks pre-MW ablation & $5 / 90(6 \%)$ \\
\hline Performed in the same setting with MW ablation & $5 / 90(6 \%)$ \\
\hline Not performed & $80 / 90(78 \%)$ \\
\hline \multicolumn{2}{|l|}{ Number of tumours ablated per procedure } \\
\hline Median & 1 (range, 1-6) \\
\hline 1 lesion & $40(67 \%)$ \\
\hline 2 lesions & $16(27 \%)$ \\
\hline 3 lesions & $6(10 \%)$ \\
\hline$>_{3}$ lesions & $2(3 \%)$ \\
\hline \multicolumn{2}{|l|}{ AJCC stage at initial diagnosis } \\
\hline I & $2(4 \%)$ \\
\hline II & $9(18 \%)$ \\
\hline III & $18(36 \%)$ \\
\hline IV & $21(42 \%)$ \\
\hline \multicolumn{2}{|l|}{ Liver metastases at initial diagnosis } \\
\hline Yes & $19(38 \%)$ \\
\hline No & $31(62 \%)$ \\
\hline \multicolumn{2}{|l|}{$\begin{array}{l}\text { Pulmonary progression outside ablation zone post-MWA } \\
\text { (in same and/or contralateral lung) }\end{array}$} \\
\hline Yes & $9(18 \%)$ \\
\hline No & $41(82 \%)$ \\
\hline \multicolumn{2}{|l|}{ Extrapulmonary progression of disease after MWA } \\
\hline Yes & $32(64 \%)$ \\
\hline No & $18(36 \%)$ \\
\hline
\end{tabular}

Table 5 Pre-MWA therapies received

\begin{tabular}{lr}
\hline Pre-MWA therapy & Incidence \\
\hline Prior lung surgery & \\
Yes & $13(26 \%)$ \\
No & $37(74 \%)$ \\
Prior lung ablation & $16(32 \%)$ \\
Yes & $34(68 \%)$ \\
No & $3(6 \%)$ \\
Multiple prior ablations (range, $2-8)$ & \\
Prior chemotherapy / target therapy received & 2 \\
$\quad$ Median chemotherapy|target therapy lines received & $16(32 \%)$ \\
1 line & $21(42 \%)$ \\
2 lines & $13(26 \%)$ \\
Z3 lines & $28(56 \%)$ \\
Prior hepatic artery infusion pump therapy & $22(44 \%)$ \\
Yes &
\end{tabular}

\begin{tabular}{lr} 
Table 5 continued. & Incidence \\
\hline Pre-MWA therapy & $47(94 \%)$ \\
\hline Prior primary tumor resection & $3(6 \%)$ \\
Yes & \\
No & $31(62 \%)$ \\
Prior liver resection & $19(38 \%)$ \\
Yes & \\
No & $16(32 \%)$ \\
Prior liver ablation & $34(68 \%)$ \\
$\quad$ Yes & \\
No & \\
Other prior therapies for metastatic disease (radiotherapy, resection or ablation of other tumor sites, & \\
radioembolization, intraperitoneal chemotherapy) & $16(32 \%)$ \\
$\quad$ Yes & $34(68 \%)$ \\
No & \\
Received only line of systemic chemotherapy + primary tumor resection \pm chemoradiotherapy and no \\
other treatments before MWA \\
$\quad$ Yes \\
No & $7(14 \%)$ \\
$\quad$ & $43(86 \%)$ \\
\hline
\end{tabular}

Primary Technique Efficacy Rate, LTP Rate and LTPFS

Median tumor size was $1 \mathrm{~cm}$ (range $0.3-3.2 \mathrm{~cm}$ ). Primary technique efficacy rate was 81/90 (90\%). Nine tumors progressed after MWA resulting in LTP rate of 9/90 (10\%) during the entire study follow-up. LTP occurred 3.9-21.8 months after the initial MWA. One-, 2- and 3-year LTPFS were: 93, 86 and 86\%, respectively. Median time to LTP was not reached (Table 7).

LTP occurred at ablation margin or directly adjacent to the ablation zone in 6/9 (66\%) LTPs. LTP within the ablation zone occurred in 3/9 (33\%) of ablations, all detected on CT imaging: one stable in size cavitary ablation zone became filled in, forming spiculated mass with pleural tethering, micronodularities and peribronchial thickening; another ablation zone showed considerable increase in solid component centrally; third ablation zone demonstrated gradual increase in size.

New focal FDG-avidity within the ablation zone consistent with LTP occurred in 4/9

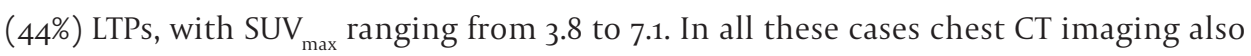
demonstrated suspicious increased ablation zone or focal nodularity adjacent to it.

Minimal Ablation Margin

Median minimal ablation margin size was $5 \mathrm{~mm}$ (range o-19 mm). Measurement of the minimal margin was feasible in 86/90 (96\%) ablations. In 4/90 (4\%) ablations it could not be calculated due to bleeding obscuring the ablation zone (in $2 \%$ ) or confluent ablation zones (in $2 \%$ ). Minimal margin of at least $5 \mathrm{~mm}$ was achieved in $48 / 86$ (56\%) of measurable ablation zones. Fourteen out of eighty-six (16\%) of metastases were pleural-based and minimal margin was considered o mm. Minimal margin size is described in Table 6. 
Table 6 Ablation technical parameters

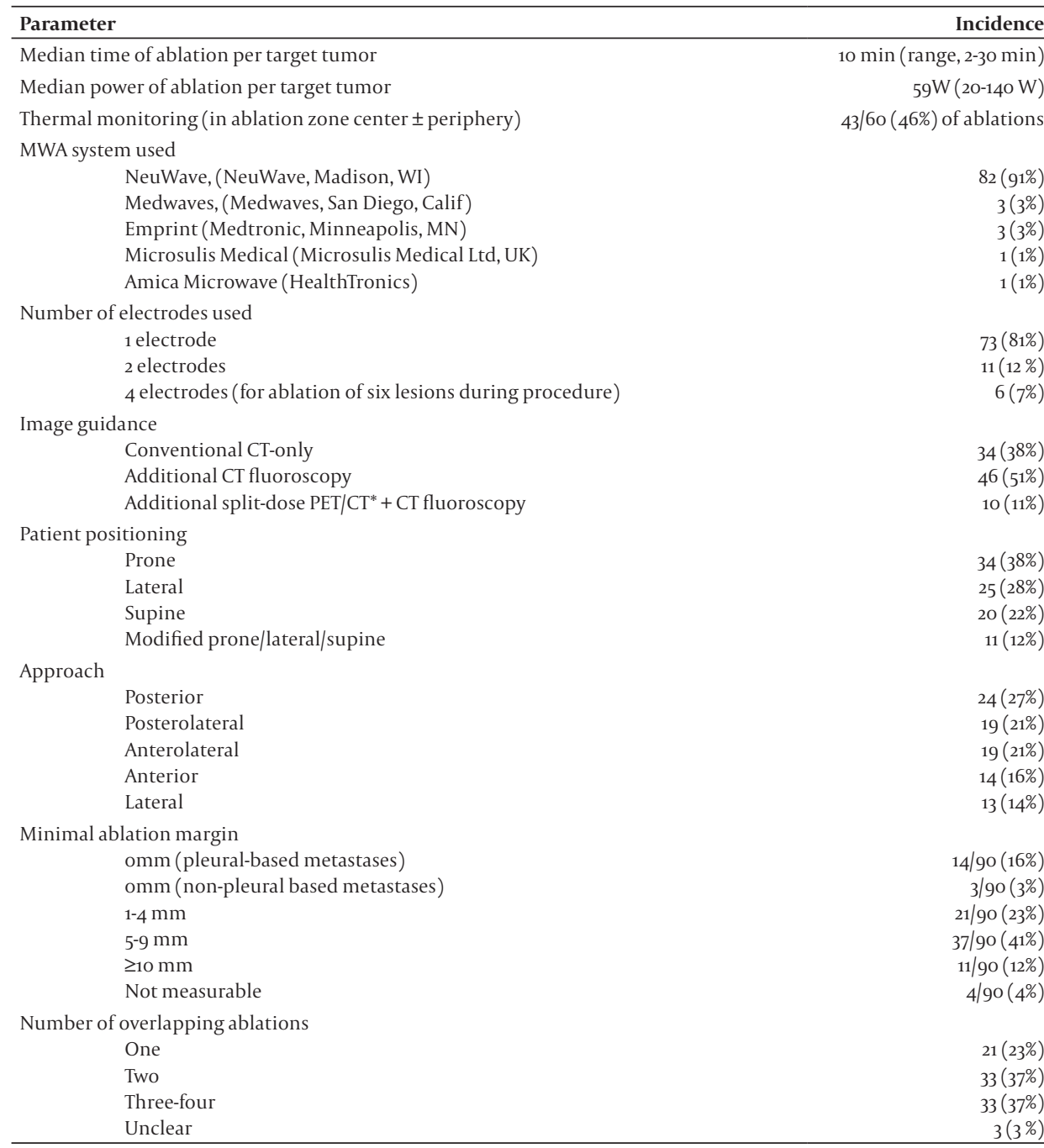

*Split - dose ${ }^{8}$ F-FDG PET/CT is a technique for PET/CT-guided ablation that permits target tumor localization and (1) $\mathrm{mCi}(444 \mathrm{MBq})$ is administered in two aliquots: a $4-\mathrm{mCi}(148-\mathrm{MBq})$ target/imaging dose is administered $30-60$ minutes before the ablation followed by an 8-mCi (296-MBq) administered immediately after the ablation with images obtained 30 minutes later. The higher dose overcomes background signal of the first lower dose and allows assessment of metabolic activity of the ablation zone and detection of residual hypermetabolic signal representing untreated residual
Table 7 Local tumor control, prognosticators of local tumor progression-free survival (LTPFS) and description of lung progression-free survival (PFS)

\begin{tabular}{|c|c|c|}
\hline \multirow{2}{*}{\multicolumn{3}{|c|}{ Lung PFS (in same and/or contralateral lung) }} \\
\hline & & \\
\hline & Median (inside and outside ablation zone) & 8.8 months $(95 \% \mathrm{CI}, 7-12.7)$ \\
\hline & Median (outside ablation zone-only) & 10 months \\
\hline & 1-year lung PFS & $40 \%(95 \% \mathrm{CI}, 27-53 \%)$ \\
\hline & 2-and 3 -year lung PFS & $15 \%(95 \% \mathrm{CI}, 7-27 \%)$ \\
\hline \multicolumn{3}{|c|}{ Local tumor control } \\
\hline & Local tumor progression (LTP) rate & $9 / 90$ (10\%), $95 \% \mathrm{CI}, 5-19 \%$ \\
\hline & Median LTPFS & Not reached \\
\hline & 1-year LTPFS & $93 \%(95 \% \mathrm{CI}, 85-97 \%)$ \\
\hline & 2-and 3 -year LTPFS & $86 \%(95 \% \mathrm{CI}, 75-93 \%)$ \\
\hline \multicolumn{3}{|c|}{ Prognosticators of LTPFS } \\
\hline Minimal a & lation margin size & $\mathrm{p}<0.001, \mathrm{SHR}=0.18,95 \% \mathrm{CI}, 0.08-0.42$ \\
\hline & LTP rate for ablations with o mm margin & $6 / 17(35 \%, 95 \% \mathrm{CI}, 12-58 \%)$ \\
\hline & LTP rate for ablations with 1-4 $\mathrm{mm}$ margin & $3 / 21(14 \%, 95 \% \mathrm{CI}, 0-29 \%)$ \\
\hline & LTP rate for ablations with $\geq 5 \mathrm{~mm}$ margin & o/48(0\%) \\
\hline & Cumulative 1-year LTP hazard for non-PB with margin $<5 \mathrm{~mm}$ & $14 \%(95 \% \mathrm{CI}, 4-43 \%)$ \\
\hline & Cumulative 2-year LTP hazard for non-PB with margin $<5 \mathrm{~mm}$ & $23 \%(95 \% \mathrm{CI}, 8-64 \%)$ \\
\hline & Cumulative 2 -year LTP hazard for non-PB with margin $\geq 5 \mathrm{~mm}$ & o \\
\hline Tumor loc & tion (pleural-based (PB) vs. non-PB) & $\mathrm{p}=0.002, \mathrm{SHR}=7.7$ \\
\hline & Cumulative 1-year LTP hazard for PB metastases & $25 \%(95 \% \mathrm{CI}, 8-77 \%)$ \\
\hline & Cumulative 2-year LTP hazard for PB metastases & $47 \%(95 \% \mathrm{CI}, 19-100 \%)$ \\
\hline & Cumulative 1-year LTP hazard of non-PB metastases & $4 \%(95 \% \mathrm{CI}, 1-13 \%)$ \\
\hline & Cumulative 2-year LTP hazard of non-PB metastases & $7 \%(95 \% \mathrm{CI}, 3-22 \%)$ \\
\hline Tumor siz & & $\mathrm{p}=0.001, \mathrm{SHR}=5.38,95 \% \mathrm{Cl}=1.9-15$ \\
\hline & LTP rate for tumours $\geq 1 \mathrm{~cm}$ in size & $19 \%(95 \% \mathrm{CI}, 8 \%-30 \%)$ \\
\hline & LTP rate for tumours $<1 \mathrm{~cm}$ in size & $0 / 43(0 \%)$ \\
\hline & LTP rate for tumours $\geq 1 \mathrm{~cm}$ with $\mathrm{MM}<5 \mathrm{~mm}$ & $41 \%(95 \% \mathrm{CI}, 20 \%-62 \%)$ \\
\hline & LTP rate for tumours $\geq 1 \mathrm{~cm}$ with $M M \geq 5 \mathrm{~mm}$ & $0 / 25(0 \%)$ \\
\hline
\end{tabular}

SHR-sub-hazard ratio

Prognosticators of LTPFS in Univariate Analysi

Minimal ablation margin size. LTPFS was statistically significantly associated with minimal margin size ( $p<0.001$ ) (Table 7 ). LTP was observed only for tumors ablated with minimal margin $<5 \mathrm{~mm}$, with LTP rate of $24 \%$ (Table 7 ).

Tumor location (pleural vs. non-pleural-based). Pleural-based metastases had 7.7 times higher LTP risk compared to non-pleural-based metastases $(\mathrm{p}=0.002)$. One and twoyear cumulative LTP hazard for non-pleural vs. pleural-based metastases was $4 \%$ and $7 \%$ versus $25 \%$ and $47 \%$, respectively (Table 7 , Figure 4 ). When analyzing non-pleural based metastases alone, the minimal ablation margin retained significance as a predictor of LTP on univariate analysis ( $\mathrm{p}<0.001$ ) (Table 7, Figure 5).

Tumor size. LTPFS was statistically significantly associated with tumor size $(\mathrm{p}=0.001)$ : LTP was only observed after MWA of tumors $\geq 1 \mathrm{~cm}$, with LTP rate of $19 \%$ (Table 7). LTP rate of 
tumors $>1 \mathrm{~cm}$ in size was $41 \%$ when minimal margin was $<5 \mathrm{~mm}$ compared to $0 \%$ when minimal margin was $\geq_{5} \mathrm{~mm}$ ( $\mathrm{p}=0.0004$, Figure 6 ).

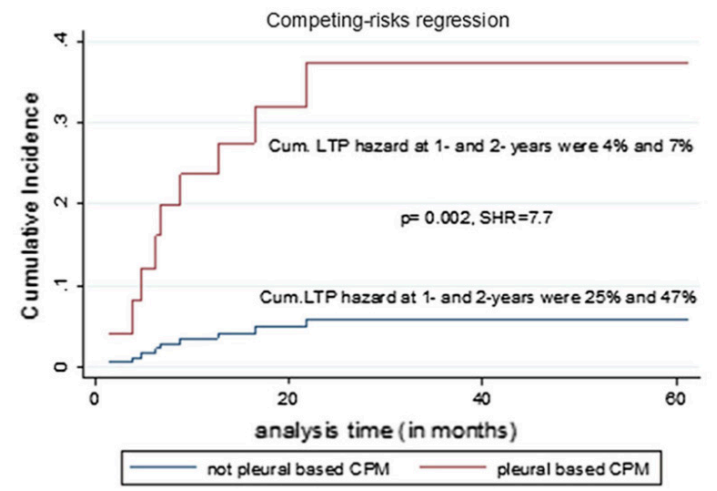

Figure 4 Cumulative (Cum.) local tumor progression (LTP) hazard of non-pleural-based and pleuralbased colorectal cancer pulmonary metastases (CPM). The figure demonstrated more than 7 times increased LTP hazard for pleural-based metastases, compared to non-pleural-based.

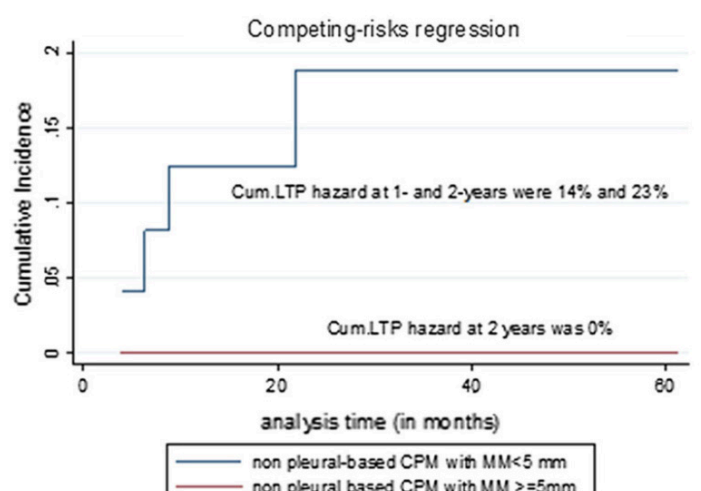

Figure ${ }_{5}$ Cumulative (Cum.) local tumor progression (LTP) hazard of non-pleural-based colorectal cancer pulmonary metastases (CPM) treated with $<5 \mathrm{~mm}$ and $\geq_{5} \mathrm{~mm}$ minimal ablation margin (MM). There was no LTP in non-pleural metastases, treated with $\geq 5 \mathrm{~mm} \mathrm{MM}$, compared to 23\% 2-year cumulative LTP hazard for metastases treated with $<5 \mathrm{~mm} \mathrm{MM}$.

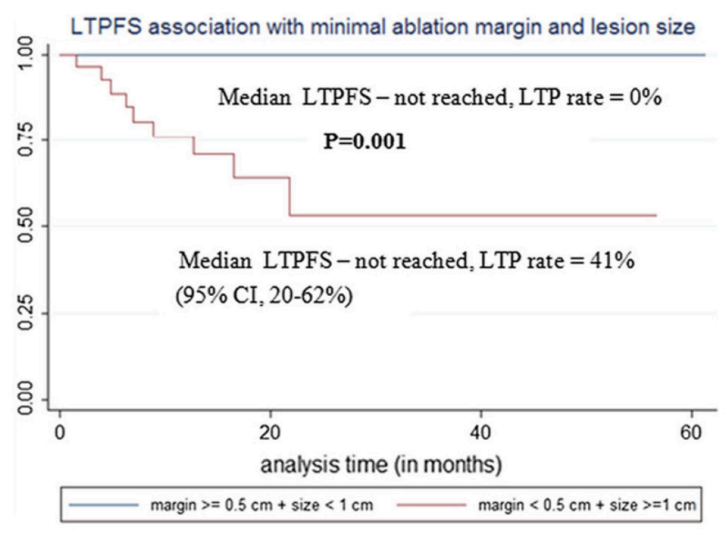

Figure 6 Local tumor progression-free survival (LTPFS) association with metastasis size and minimal ablation margin

Overall and Cancer-Specific survival

Median OS was 58.6 months, and median cancer-specific survival was not reached (Table 7). Median OS for the patients without LTP was 45.2 months, and not reached for patients with $\operatorname{LTP}(\mathrm{HR}=0.63, \mathrm{p}=0.54)$.

Three out of sixteen (19\%) of deaths was not cancer-related. They included sepsis and multi-organ failure in a patient with retroperitoneal abscess (4.7 months post-MWA), respiratory failure due to pneumonia (18 months post-MWA) and death of other cause 11 months post-MWA, with no evidence of oncologic disease in one patient (negative whole body ${ }^{18} \mathrm{~F}-\mathrm{FDG}$ PET/CT 2 months before death). One out of sixteen (6\%) of patients was lost to follow-up 13 months before death.

Management of LTP and Assisted LTPFS

Repeat MWA was offered in 2/9 (22\%) LTPs and one residual tumor. Assisted LTPFS was not reached. Repeat MWA for residual tumor increased local tumor control with MWA from 1.5 to 23.7 months in one patient (after one repeat MWA; however, patient had subsequent LTP which was aggressively re-treated with RFA using 3 electrodes and 4 overlapping ablations), from 8.83 months to 75.5 months in second patient (two additional MWAs, no further LTP) and from 3.9 to 19.37 months in third patient (single additional MWA, no further LTP). Resulting secondary technique efficacy rate, accounting for all repeat MWAs was $83 / 90$ (92\%). LTPs in the rest of patients were managed with: systemic chemotherapy in $4 / 9(44 \%)$, lung resection in $1 / 9(11 \%)$ and lung radiotherapy in $1 / 9(11 \%)$. 
Post-ablation Patient Management, Lung and Extrapulmonary Disease Progression Six out of fifty (12\%) of patients had no lung progression (inside and/or outside the ablation zone). Lung progression outside ablation zone was ablated in 14/41 (34\%) of patients. Median overall lung progression-free survival was 8.8 months (Table 7, Figure 7).

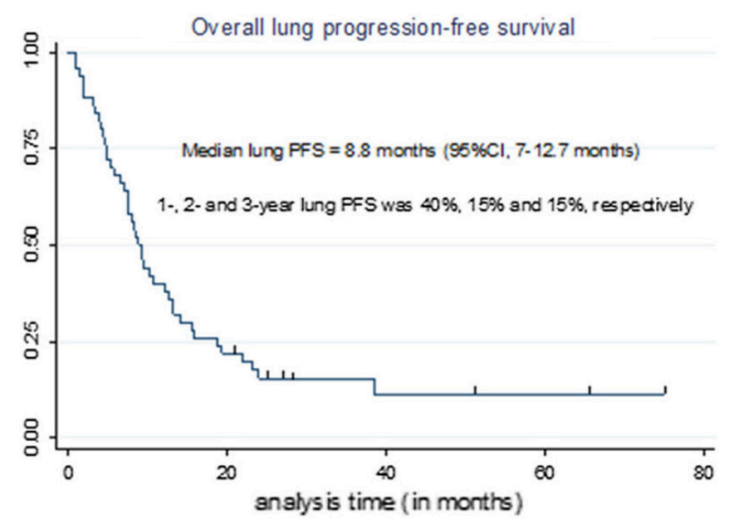

Figure 7 Overall lung progression free-survival (PFS, inside and/or outside the ablation zone)

Thirty-two out of fifty (64\%) patients had extra-pulmonary disease progression postMWA (with pulmonary progression in 29/32 (91\%), and without in 3/32 (9\%) patients).

Three out of fifty $(6 \%)$ of patients were disease-free post-lung ablation and were offtreatment for 25 months, 65.4 months and 74.9 months. Post-MWA therapies are presented in Table 8.

Cancer-Specific Survival Prognosticators

Pre-MWA CEA level $>10 \mathrm{ng} / \mathrm{ml}(\mathrm{p}=0.046$, Table 9 , Figure 8 ) and $\geq 3$ pre-MWA chemotherapy \pm target therapy lines were associated with cancer-specific survival on univariate analysis $(\mathrm{p}=0.02$, Table 9 , Figure 9$)$.

Prior lung or liver resection and lung as the first site of metastatic disease were not associated with cancer-specific survival (Table 9).

Small number of deaths and intermediate follow-up time precluded multivariate analysis of any predictors.
Table 8 Post-microwave ablation (MWA) therapies received

\begin{tabular}{|c|c|}
\hline Post-MWA therapy & Incidence \\
\hline \multicolumn{2}{|l|}{ Systemic chemotherapy } \\
\hline Yes. Number of regimens received: & $37(74 \%)$ \\
\hline 1 regimen & $12 / 37(32 \%)$ \\
\hline 2 regimens & $12 / 37(32 \%)$ \\
\hline$\geq 3$ regimes & $13 / 37(35 \%)$ \\
\hline No & $13(26 \%)$ \\
\hline \multicolumn{2}{|c|}{ Target therapy (EGFR inhibitors/antibody, anti-VEGF, tyrosine kinase inhibitors) } \\
\hline Yes & $29(58 \%)$ \\
\hline No & $21(42 \%)$ \\
\hline \multicolumn{2}{|l|}{ Lung ablation } \\
\hline Yes & $19(38 \%)$ \\
\hline No & $31(62 \%)$ \\
\hline \multicolumn{2}{|l|}{ Lung resection } \\
\hline Yes & $5(10 \%)$ \\
\hline No & $45(90 \%)$ \\
\hline \multicolumn{2}{|c|}{$\begin{array}{l}\text { Other therapies (radiotherapy, resection or ablation of the other tumor sites, } \\
\text { radioembolization, hepatic artery infusion pump therapy, immunotherapy) }\end{array}$} \\
\hline Yes & \\
\hline No & $22(44 \%)$ \\
\hline \multicolumn{2}{|c|}{$\begin{array}{l}\text { Patients off-treatment after MWA due to no evidence of disease, low volume of disease or due } \\
\text { side effects/comorbidities }\end{array}$} \\
\hline Yes & $5(10 \%)$ \\
\hline No & $45(90 \%)$ \\
\hline
\end{tabular}

Table 9 Overall survival, cancer-specific survival and prognosticators of them

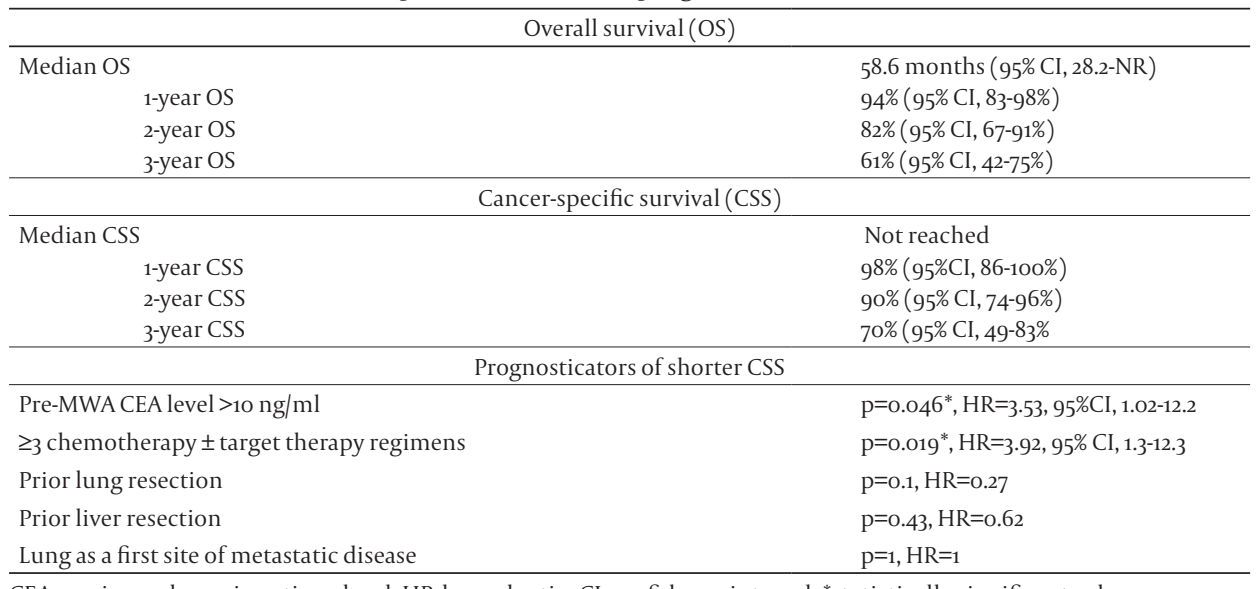

CEA-carcinoembryonic antigen level, HR-hazard ratio, CI-confidence interval, "statistically significant values 


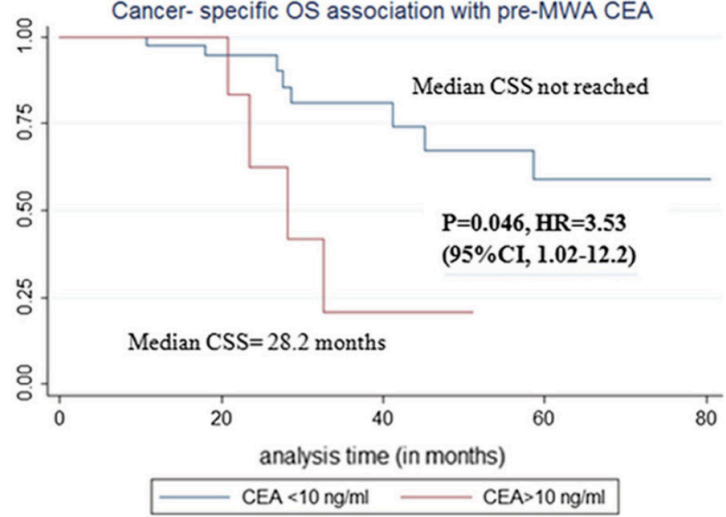

Figure 8 Cancer-specific survival (CSS) association with pre-MWA carcinoembryonic antigen (CEA) level

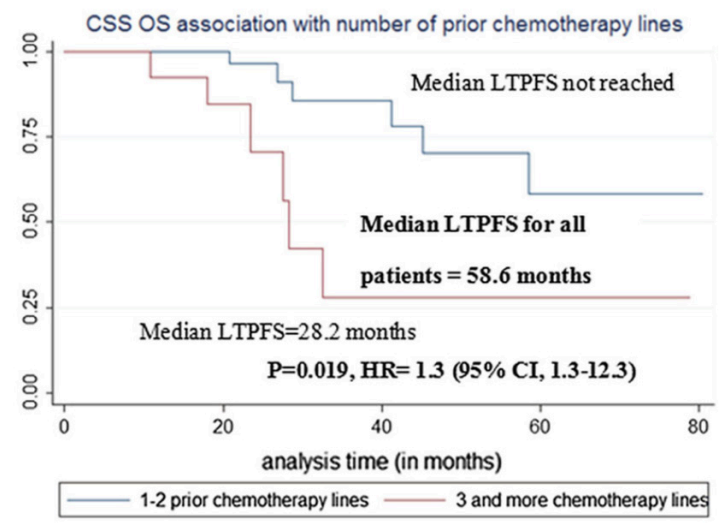

Figure 9 Cancer -specific survival (CSS) association with number of pre-MWA chemotherapy \pm target therapy lines

\section{Side Effects and Complications}

Incidence of side effects was $12 / 60$ (20\%). Minor complications rate was $23 / 60(38 \%)$ and included pneumothorax, requiring thoracostomy. Major complications rate was $8 / 60$ (13\%); $75 \%$ of major complications included pneumothoraces requiring prolonged hospitalization (Table 10).
Table 10 Side effects, minor and major complications following lung MW ablation.

\begin{tabular}{|c|c|}
\hline Adverse event & Incidence \\
\hline Side effects (asymptomatic pneumothoraces, no treatment) & $12 / 60(20 \%)$ \\
\hline Minor complications (pneumothoraces, requiring thoracostomy), overal incidence & $23 / 60(38 \%)$ \\
\hline Moderate pneumothoraces & $12 / 23(52 \%)$ \\
\hline Enlarging (hemo)pneumothoraces & $4 / 23(17 \%)$ \\
\hline Large pneumothoraces & $1 / 23(4 \%)$ \\
\hline $\begin{array}{l}\text { Small recurrent/circumferential/symptomatic pneumothorax or small pneumothorax with } \\
\text { persistent air leak/ small pleural effusion/pneumomediastinum }\end{array}$ & $5 / 23(22 \%)$ \\
\hline Small asymptomatic non-circumferential pneumothorax, no admission & $1 / 23(4 \%)$ \\
\hline Median length of stay of patients with pneumothorax & 1 day (range, $0-7)$ \\
\hline Major complications, overal incidence & $8 / 60(13 \%)$ \\
\hline $\begin{array}{l}\text { Thoracostomy, requiring prolonged hospitalization (3-7 days) for persistent air leak, } \\
\text { desaturation, severe pain or intra-parenchymal bleeding with large hemopneumothorax; } \\
\text { Aspergilloma formation in the ablation zone cavity } 2 \text { months after MWA. This became chronic }\end{array}$ & $6 / 60(10 \%)$ \\
\hline $\begin{array}{l}\text { pulmonary aspergillosis requiring hospitalization for } 2 \text { days with prolonged treatment with } \\
\text { voriconazole ( } 6 \text { weeks } 200 \mathrm{mg} \mathrm{p} / \mathrm{os} \text { every } 12 \text { hours and subsequent } 6 \text { weeks of } 100-400 \mathrm{mg} \text { IVPB } \\
\text { every } 12 \text { hours); }\end{array}$ & $1 / 60(2 \%)$ \\
\hline $\begin{array}{l}\text { Pleuritic pain } 12 \text { days after MWA with underlying pneumonia, empyema, bronchopleural } \\
\text { fistula and dehydration requiring hospitalization for 1o days with intravenous antibiotics }\end{array}$ & $1 / 60(2 \%)$ \\
\hline $\begin{array}{l}\text { (combination of piperacillin and tazzobactam), infusion therapy and analgesics } \\
\text { (fentanyl and porphine) After discussion with thoracic surgery service the underlying }\end{array}$ & \\
\hline $\begin{array}{l}\text { (fentanyl and morphine). After discussion with thoracic surgery service, the underlying } \\
\text { bronchopleural fistula was successfully managed with prolonged thoracostomy for } 5\end{array}$ & \\
\hline $\begin{array}{l}\text { months in the outpatient setting without further sequelae. } \\
\text { mong }\end{array}$ & \\
\hline Deaths within go days post-MWA & $\mathrm{o} / 60(0 \%)$ \\
\hline
\end{tabular}

MWA-microwave ablation: IVPB-piggy bag (short-term or secondary infusion)

\section{DISCUSSION}

The LTP rate of $10 \%$ in this cohort compared favorably to reported 12-26\% LTP-rates of prior pulmonary tumors MWA series [33-35, 37]. This may be attributed to the relatively small tumor size and the creation of minimal margin $\geq_{5} \mathrm{~mm}$ in large number of patients $(56 \%)$. We found strong association between tumor size $(\mathrm{p}=0.001)$, minimal ablation margin $(\mathrm{p}<0.001)$, lesion location $(\mathrm{p}=0.002)$ and LTP. Small LTP number precluded multivariate analysis.

All LTPs occurred in tumors $\geq 1 \mathrm{~cm}$, ablated with minimal margin $<5 \mathrm{~mm}$, with $41 \%$ LTP rate for tumors with both these factors $(\mathrm{p}=0.0004)$. This observation supports the value of the minimal margin as independent predictor of LTP [7, 22, 31, 38, 39]. Tumor size remains a limiting factor for the widespread use of ablation regardless of energy used $[23,40-42]$.

Pleural-based tumors had more than seven times higher LTP risk. Minimal ablation margin remained significant predictor of LTP for non-pleural-based metastases.

One-, 2- and 3-year survival rates of 94, 84 and 60\% compared favorably to reported ranges 
of $84-95,56-72$ and $35-65$, respectively [43].

LTP did not affect overall survival $(\mathrm{p}=0.54$ ). The majority of patients received systemic chemotherapy and/or targeted therapy post-MWA; more than a third of patients had subsequent lung ablation or lung resection for progression. Therefore, overall survival could not be solely attributed to lung ablation, but rather to the overall therapeutic management of oligometastatic disease. Local therapy for pulmonary metastases significantly prolonged 3-year survival in patients receiving adjuvant radiotherapy or ablation, compared to those treated with chemotherapy alone (88 vs. $33 \%$, respectively) [44].

Pre-MWA CEA level and number of prior chemotherapy and target therapy regimens predicted cancer-specific survival. CEA association with survival can be explained by CEA circulating cancer cell death inhibition and activation of cell adhesion-related molecules [45] that has been reported after metastasectomy of CRC lung metastases $[46,47]$.

Thoracostomy rate of $38 \%$ is higher than reported after RFA (13-33\%) [43]. This may be related to our clinical protocol of thoracostomy for any circumferential pneumothorax even in asymptomatic patients. The rate of moderate/large or enlarging pneumothorax was $28 \%$, requiring prolonged hospitalization in $10 \%$ of procedures. The overall major complication rate of $13 \%$ is comparable to the $20 \%$ rate in prior lung MWA series $[48,49]$. Approximately a third of patients underwent MWA for progression of disease after lung metastatectomy and only $11 \%$ of the tumors were biopsy-proven, since radiological diagnosis of lung metastases is generally reliable [50].

There currently is an ongoing debate on the impact of local therapy and pulmonary metastasectomy on survival in this population. Most of the evidence supporting complete removal of pulmonary metastases originated from surgical series of wellselected patients with favorable characteristics (a few or small size metastases) without any comparison to patients that were not resected [47,51]. The lack of randomized data and comparative studies make conclusions about the impact of resection and locoregional therapies difficult [51]. Lung metastatectomy can improve survival; however, recurrence rates are high with repeat thoracotomy being associated with higher morbidity, whereas lobectomy offers no survival benefit when compared to limited sub-lobar or wedge resections [51]. Therefore, ablation could be advocated to be a preferable local therapy for small tumors, which can be ablated with sufficient margins.
The study had several limitations including its retrospective nature, the small number of patients and the intermediate length of follow-up, precluding long-term estimations. Another limitation is the lack of pathologic complete ablation confirmation, a common limitation of interventional oncology therapies. The impact of MWA device choice on local tumor control was limited since $91 \%$ of all tumors were ablated using NeuWave device. Known genetic signatures and predictive biomarkers were not available for the entire cohort of patients and could not be assessed as potential factors impacting outcomes [39, 52-55].

To summarize, this work indicated the efficacy of MWA that compared favorably to prior results of thermal ablation in terms of local tumor control, local progression-free survival and overall survival. Factors associated with LTPFS and cancer-specific survival were identified and described. Further validation is certainly needed in larger studies with longer follow-up to better define the role of MWA in the management of CRC metastatic disease. 


\section{REFERENCES}

1. Siegel RL, Miller KD, Fedewa SA, Ahnen DJ, Meester RGS, Barzi A, et al. Colorectal cancer statistics, 2017. CA Cancer J Clin. 2017; 67(3):177-93.

2. Cook AD, Single R, McCahill LE. Surgical resection of primary tumors in patients who presen with stage IV colorectal cancer: an analysis of surveillance, epidemiology, and end results data, 1988-2000. Ann Surg Oncol. 2005; 12(8):637-45.

3. Kobayashi H, Mochizuki H, Sugihara K, Morita T, Kotake K, Teramoto T, et al. Characteristics of recurrence and surveillance tools after curative resection for colorectal cancer: a multicenter study. Surgery. 2007;141(1):67-75

4. Mitry E, Guiu B, Cosconea S, Jooste V, Faivre J, Bouvie AM. Epidemiology, management and prognosis of colorectal cancer with lung metastases: a 30-year population-based study. Gut. 2010; 59(10):1383-8.

5. Siegel R, Desantis C, Jemal A. Colorectal cancer statistics, 2014. CA Cancer J Clin. 2014; 64(2):104-17

6. Fiorentino F, Hunt I, Teoh K, Treasure T, Utley M. Pulmonary metastasectomy in colorectal cancer a systematic review and quantitative synthesis. J R Soc Med. 2010; 103(2):60-6.

7. Shady W, Petre EN, Gonen M, Erinjeri JP, Brown KT, Covey AM, et al. Percutaneous radiofrequency ablation of colorectal cancer liver metastases: factors affecting outcomes-a 10-year experience at a single center. Radiology. 2016;278(2):601-11.

8. Ruers T, Van Coevorden F, Punt CJ, Pierie JE, BorelRinkes I, Ledermann JA, et al. Local treatment of unresectable colorectal liver metastases: results of a randomized phase II trial. J Natl Cancer Inst. 2017:109(9):djxo15.

9. Solbiati L, Ahmed M, Cova L, Ierace T, Brioschi M, Goldberg SN. Small liver colorectal metastase treated with percutaneous radiofrequency ablation: local response rate and long-term survival with up to 10-year follow-up. Radiology. 2012;265(3):958-68.

10. Embun R, Fiorentino F, Treasure T, Rivas JJ, Molins L. Pulmonary metastasectomy in colorectal cancer: a prospective study of demography and clinical characteristics of 543 patients in the Spanish colorectal metastasectomy registry (GECMP-CCR). BMJ Open. 2013;3(5):eoo2787.

11. Gonzalez M, Poncet A, Combescure C, Robert J, Ris HB, Gervaz P. Risk factors for survival after lung metastasectomy in colorectal cancer patients: a systematic review and meta-analysis. Ann Surg Oncol. 2013;20(2):572-9.

12. Rios A, Galindo PJ, Torres J, Roca MJ, Robles R, Lujan JA, et al. Factors causing early relapse after lung metastasis surgery. Eur J Cancer Care (Engl). 2007:16(1):26-32.

13. Pfannschmidt J, Hoffmann $\mathrm{H}$, Dienemann $\mathrm{H}$. Reported outcome factors for pulmonary resection in metastatic colorectal cancer. J Thorac Oncol. 2010;5(6 Suppl 2):S172-8

14. Welter S, Jacobs J, Krbek T, Krebs B, Stamatis G. Long-term survival after repeated resection of pulmonary metastases from colorectal cancer. Ann Thorac Surg. 2007;84(1):203-10.

15. Simon CJ, Dupuy DE, DiPetrillo TA, Safran HP, Grieco CA, Ng T, et al. Pulmonary radiofrequency ablation: long-term safety and efficacy in 153 patients. Radiology. 2007;243(1):268-75.

16. Sofocleous CT, May B, Petre EN, Gonen M, Thornton RH, Alago W, et al. Pulmonary thermal ablation in patients with prior pneumonectomy. AJR Am ] Roentgenol. 2011;196(5):W6o6-12.

17. Hess A, Palussiere J, Goyers JF, Guth A, Auperin A, de Baere T. Pulmonary radiofrequency ablation in patients with a single lung: feasibility, efficacy, and tolerance. Radiology. 2011;258(2):635-42.

18. Livraghi T, Solbiati L, Meloni F, Ierace T, Goldberg SN, Gazelle GS. Percutaneous radiofrequency ablation of liver metastases in potential candidates for resection: the "test-of-time approach". Cancer. 2003;97(12):3027-35.

19. Hiraki T, Gobara H, lishi T, Sano Y, Iguchi T, Fujiwara $H$, et al. Percutaneous radiofrequency ablation for pulmonary metastases from colorectal cancer: midterm results in 27 patients. J Vasc Interv Radiol (JVIR). 2007;18(10):1264-9.

20. Yamakado K, Hase S, Matsuoka T, Tanigawa N, Nakatsuka A, Takaki H, et al. Radiofrequency ablation for the treatment of unresectable lung metastases in patients with colorectal cancer: a multicenter study in Japan. J Vasc Interv Radio (JVIR). 2007;18(3):393-8.

21. De Baere T, Auperin A, Deschamps F, Chevallier $F$ Gaubert Y, Boige V, et al. Radiofrequency ablation is a valid treatment option for lung metastases: experience in 566 patients with 1037 metastases. Ann Oncol. 2015;26(5):987-91.

22. Yan TD, King J, Sjarif A, Glenn D, Steinke K, Morris DL. Percutaneous radiofrequency ablation of pulmonary metastases from colorectal carcinoma: prognostic determinants for survival. Ann Surg Oncol. 2006; ;3(11):1529-37.

23. Vogl T], Naguib NN, Gruber-Rouh T, Koitka K Lehnert T, Nour-Eldin NE. Microwave ablation therapy: clinical utility in treatment of pulmonary metastases. Radiology. 2011;261(2):643-51.

24. Egashira Y,Singh S, Bandula S, Illing R. Percutaneous high energy microwave ablation for the treatmen of pulmonary tumors: a retrospective singlecenter experience. J Vasc Interv Radiol (JVIR). 2016;27(4):474-9.

25. Petre EN, Jia X, Thornton RH, Sofocleous CT, Alago
W, Kemeny NE, et al. Treatment of pulmonary colorectal metastases by radiofrequency ablation. Clin Colorectal Cancer. 2013;12(1):37-44.

26. Belfiore G, Moggio G, Tedeschi E, Greco M, Cioffi $R$, Cincotti F, et al. CT-guided radiofrequency ablation: a potential complementary therapy for patients with unresectable primary lung cancer-a preliminary report of 33 patients. AJR Am I Roentgenol. 2004;183(4):1003-11.

27. Sacks D, McClenny TE, Cardella JF, Lewis CA. Society of Interventional Radiology clinical practice guidelines. J Vasc Interv Radiol (JVIR). 2003;14(9 Pt 2):S199-202.

28. King J, Glenn D, Clark W, Zhao J, Steinke K, Clingan P, et al. Percutaneous radiofrequency ablation of pulmonary metastases in patients with colorectal cancer. Br J Surg. 2004;91(2):217-23.

29. Steinke K, King J, Glenn DW, Morris DL Percutaneous radiofrequency ablation of lung tumors with expandable needle electrodes: tips from preliminary experience. AJR Am J Roentgenol. 2004;183(3):605-11.

30. Sofocleous CT, Sideras P, Petre EN, Solomon SB Ablation for the management of pulmonary malignancies. AJR Am J Roentgenol. 2011;197(4):W581-9.

31. Mouli SK, Kurilova I, Sofocleous CT, Lewandowski RJ. The role of percutaneous image-guided thermal ablation for the treatment of pulmonary malignancies. AJR Am J Roentgenol. 2017;209(4):740-51.

32. Lubner MG, Brace CL, Hinshaw JL, Lee FT Ir. Microwave tumor ablation: mechanism of action, clinical results, and devices. J Vasc Interv Radiol (JVIR). 2010;21(8 Suppl):S192-203.

33. Vogl TJ, Eckert R, Naguib NN, Beeres M, GruberRouh T, Nour - Eldin NA. Thermal ablation of colorectal lung metastases: retrospective 
comparison among laser-induced thermotherapy, radiofrequency ablation, and microwave ablation. AJR Am J Roentgenol. 2016;207(6):1340-9.

34. Wolf FJ, Grand DJ, Machan JT, Dipetrillo TA, MayoSmith WW, Dupuy DE. Microwave ablation of lung malignancies: effectiveness, CT findings, and safety in 50 patients. Radiology. 2008;247(3):871-9.

35. Lu Q, Cao W, Huang L, Wan Y, Liu T, Cheng Q, et al. CT guided percutaneous microwave ablation of pulmonary malignancies: results in 69 cases. World J Surg Oncol. 2012;10:80.

36. Ahmed M, Solbiati L, Brace CL, Breen DJ, Callstrom MR, Charboneau JW, et al. Image-guided tumor ablation: standardization of terminology and reporting criteria-a 10-year update. Radiology. 2014;273(1):241-60.

37. Wolf FJ, Aswad B, Ng T, Dupuy DE. Intraoperative microwave ablation of pulmonary malignancies with tumor permittivity feedback control: ablation and resection study in 10 consecutive patients. Radiology. 2012;262(1):353-60.

38. Giraud P, Antoine M, Larrouy A, Milleron B, Callard P, De Rycke Y, et al. Evaluation of microscopic tumor extension in non-small-cell lung cancer for threedimensional conformal radiotherapy planning. Int J Radiat Oncol Biol Phys. 2000;48(4):1015-24.

39. Sotirchos VS, Petrovic LM, Gonen M, Klimstra DS, Do RK, Petre EN, et al. Colorectal cancer liver metastases: biopsy of the ablation zone and margins can be used to predict oncologic outcome. Radiology. 2016;280(3):949-59.

40. Steinke K, Glenn D, King J, Clark W, Zhao J, Clingan $\mathrm{P}$, et al. Percutaneous imaging-guided radiofrequency ablation in patients with colorectal pulmonary metastases: 1-year follow-up. Ann Surg Oncol. 2004;11(2):207-12.

41. Akeboshi M, Yamakado K, Nakatsuka A, Hataj

O, Taguchi O, Takao M, et al. Percutaneous radiofrequency ablation of lung neoplasms: initial therapeutic response. J Vasc Interv Radiol (JVIR). 2004; $15(5): 463-70$.

42. Simon TG, Beland MD, Machan JT, Dipetrillo T, Dupuy DE. Charlson Comorbidity Index predicts patient outcome, in cases of inoperable non-small cell lung cancer treated with radiofrequency ablation. Eur J Radiol. 2012;81(12):4167-72.

43. Lyons NJ, Pathak S, Daniels IR, Spiers A, Smart NJ. Percutaneous management of pulmonary metastases arising from colorectal cancer; a systematic review. Eur J Surg Oncol. 2015;41(11):1447-

44. Inoue Y, Miki C, Hiro J, Ojima E, Yamakado K, Takeda $\mathrm{K}$, et al. Improved survival using multi-modality therapy in patients with lung metastases from colorectal cancer: a preliminary study. Oncol Rep. 2005; ;4(6):1571-6.

45. Lee JH, Lee SW. The roles of carcinoembryonic antigen in liver metastasis and therapeutic approaches. Gastroenterol Res Pract. 2017;2017:7521987

46. McAfee MK, Allen MS, Trastek VF, Ilstrup DM, Deschamps C, Pairolero PC. Colorectal lung metastases: results of surgical excision. Ann Thorac Surg. 1992;53(5):780-5 (discussion 5-6).

47. Higashiyama M, Kodama K, Higaki N, Takami K, Murata K, Kameyama M, et al. Surgery for pulmonary metastases from colorectal cancer: the importance of pre-thoracotomy serum carcinoembryonic antigen as an indicator of prognosis. Jpn J Thorac Cardiovasc Surg. 2003;51(7):289-96.

48. Splatt AM, Steinke K. Major complications of highenergy microwave ablation for percutaneous CTguided treatment of lung malignancies: singlecentre experience after 4 years. J Med Imaging Radiat Oncol. 2015;59(5):609-16.

49. Zheng A, Wang X, Yang X, Wang W, Huang G, Gai
$\mathrm{Y}$, et al. Major complications after lung microwave ablation: a single center experience on 204 sessions. Ann Thorac Surg. 2014;98(1):243-8.

50. Nordholm-Carstensen A, Krarup PM, Jorgensen LN, Wille-Jorgensen PA, Harling H. Occurrence and survival of synchronous pulmonary metastases in colorectal cancer: a nationwide cohort study. Eur ] Cancer. 2014;50(2):447-56.

51. Chua TC, Al-Alem I, Zhao J, Glenn D, Liauw W, Morri DL. Radiofrequency ablation of concomitant and recurrent pulmonary metastases after surgery for colorectal liver metastases. Ann Surg Oncol. 2012;19(1):75-81.

52. Sofocleous CT, Garg SK, Cohen P, Petre EN, Gonen M Erinjeri JP, et al. Ki 67 is an independent predictive biomarker of cancer specific and local recurrencefree survival after lung tumor ablation. Ann Surg Oncol. 2013;20(Suppl 3):S676-83.

53. Ziv E, Erinjeri JP, Yarmohammadi H, Boas FE, Petre EN, Gao S, et al. Lung adenocarcinoma: predictive value of KRAS mutation status in assessing local recurrence in patients undergoing image guided ablation. Radiology. 2017;282(1):251-8.

54. Shady W, Petre EN, Vakiani E, Ziv E, Gonen M, Brown $\mathrm{KT}$,etal. Kras mutation is a marker of worse oncologic outcomes after percutaneous radiofrequency ablation of colorectal liver metastases. Oncotarget. 2017;8(39):6611-27.

55. Odisio BC, Yamashita S, Huang SY, Harmoush S, Kopetz SE, Ahrar K, et al. Local tumour progression after percutaneous ablation of colorectal liver metastases according to RAS mutation status. $\mathrm{Br}$ ] Surg. 2017;104(6):760-8. 


\section{PART 3}

General discussion, valorization and summary 
General Discussion,

Future Perspectives and

Conclusion 


\section{GENERAL DISCUSSION}

In this thesis, we identified factors that can improve patient selection and modify treatment approach in order to increase local tumor control and reduce morbidity following lung and liver thermal ablation as well as ${ }^{\circ} \mathrm{Y}$ radioembolization for patients with metastatic CRC.

In our literature review on liver-directed therapies for the patients with metastatic CRC, we discussed the role of interventional oncology (IO) procedures among other local and systemic therapies. Hepatectomy, which is considered the gold standard treatment for CRC liver and lung metastases, is no longer the only potentially curative treatment: thermal ablation can result in local cure in well selected patients that can undergo ablation with sufficient minimal ablation margins $[1,2]$. Interventional oncologists/ radiologists strive to achieve oncological outcomes similar to those achieved with resection, establishing the terms "resectable" and "ablatable" as synonyms [3-5] for selected patients with small CRC metastases.

Minimal ablation margin is a key modifiable procedure factor of liver $[6,7]$ and lung [8] thermal ablations. As CRC liver metastases were found to have distinct histological patterns, which impact prognosis $[9,10]$, it is important to ablate the area around the tumor to eradicate all viable tumor cells. In case insufficient minimal ablation margin is detected on immediate post-ablation scan, it may still be corrected with an immediate on-site additional ablation creating a larger ablation zone. In addition, a patient with an insufficient minimal ablation margin $(<5 \mathrm{~mm})$ may be handled by the medical oncologist and benefit from adjuvant chemotherapy after ablation.

Despite significant progress achieved in creating methods to assess the minimal ablation margin size, these still have limitations. Common limitations include an inadequate description of the methodology, lack of accountancy for non-ellipsoid tumor geometry or eccentric ablation zone, as well as lack of availability of imaging registration software [11-14]. In this thesis we utilized a manual minimal ablation margin assessment method using anatomical landmarks on pre- and post-ablation CT [6] that can be universally reproducible and do not require dedicated software. We also validated the correlation of minimal margin with local tumor progression $[6,7]$ and showed that a minimal ablation margin of $>10 \mathrm{~mm}$ was associated with no LTP within the median study follow-up of 31 months.

Incidence of biliary complications following liver thermal ablation has been reported to be within a range of $0.1-12 \%$ [15-18]. In our study, biliary complications were the most common type of major complication following liver thermal ablation. To the best of our knowledge, factors contributing to biliary complications have not been fully investigated. Although prior biliary interventions, proximity to major biliary structures and history of bilioenteric anastomoses are known risk factors for biliary complications a detailed analysis that can predispose to biliary and other major complications has been lacking [19].

We showed that several easy-to-obtain pre-treatment parameters could help to detect those patients with an increased risk of post-ablation biliary complications. The main biliary complication risk factor in our cohort was a history of prior hepatic artery infusion (HAI) chemotherapy carrying $100 \%$ sensitivity in predicting biliary complications. Additional risk factors included pre-existing biliary dilatation and bevacizumab administration within 1-month prior to ablation. Notably, our study showed that even though a minimal ablation margin of $>10 \mathrm{~mm}$ is associated with the best local tumor control, it might also contribute to the risk for biliary complications in specific high-risk patient cohorts. Thus by modifying the size of minimal ablation margin, we can actually decrease the number of biliary complications in these selected groups.

The trade-off between local tumor control and safety is arbitrary. The question of what is the acceptable local tumor control and complications rate is a subject for discussion and may end up being customized for each patient based on the long-term management goals (that greatly depend on extent of extrahepatic disease) as well as on the molecular profile of the specific disease.

There is no increased concern for biliary complications in HAI-naïve patients. These patients were safely ablated with $>10 \mathrm{~mm}$ margins without added morbidity in our study. An important challenge is to define the optimal ablation margin for the high-risk patients with prior HAI therapy. A reasonable option for safe and effective treatment of the high-risk patients with prior HAI therapy may be the creation of a 6-10 $\mathbf{~ m m ~ m i n i m a l ~}$ ablation margin. Compared to the patients, ablated with $>10 \mathrm{~mm}$ minimal margin, those ablated with 6-10 mm minimal margins had significantly lower major biliary complications rate ( $4 \%$ vs. $31 \%$ ) and a rather "acceptable" than "optimal" local tumor control rate (76\% vs. 100\%). A $24 \%$ local tumor progression rate was deemed acceptable for these high-risk patients as they can be salvaged with re-ablation and other local therapies together with adjuvant systemic therapies or additional HAI chemotherapy. Further improvement of local tumor control in these patients can be achieved with 
tissue confirmation of complete tumor necrosis through core biopsy of ablation zone center and a margin. A tumor-negative biopsy of the ablation zone combined with $5 \mathrm{~mm}$ minimal ablation margins offers $97 \%$ local tumor progression-free survival at a median 30 month follow-up [20]. Identification of viable tumor in biopsy could prompt re-ablation, adjuvant therapies and/or closer imaging follow-up. Such precaution is especially important for the high-risk group of patients with history of HAI, prior bevacizumab exposure, pre-existing biliary dilatation or biloma (on CT imaging) where the ideal minimal ablation margin would further add to the risks for biliary complication.

Although many studies have addressed the role of ${ }^{\circ} \mathbf{Y}$ radiation segmentectomy for hepatocellular carcinoma these data are scarce for liver metastatic disease. ${ }^{9} \mathrm{Y}$ radiation segmentectomy is an important new treatment option for the patients without curative treatment alternatives and not eligible for liver resection or ablation. Our preliminary findings suggest that for this patient population radiation segmentectomy of $\leq 3$ liver segments is safe and enables to achieve high tumor response rates and local tumor control. However, optimal dosimetry methodology for this procedure requires further investigation.

We were able to design a nomogram prognostic of overall survival, which can aid in improving patient selection for ${ }^{90} \mathrm{Y}$ radioembolization in the salvage setting to avoid unnecessary morbidity [21]. Our nomogram includes six easy-to-obtain parameters, widely available in an electronic patient record system. These factors include parameters related to tumor burden (metastatic liver tumor size, presence of extrahepatic disease and CEA level), tumor differentiation level as well as biochemical parameters reflecting liver damage and synthetic function. It provides good discrimination of the patients' 1-year survival. For the patients with a $\leq 10 \%$ predicted chance of 1-year survival, it is important to discuss the treatment risks and benefits with the patient to have realistic survival expectations and potentially consider other treatment options. Internal validation showed that the nomogram had good discrimination and calibration.

Realistic, unbiased outcome scores can be useful for both the patients and healthcare providers. Such a nomogram can be employed during an initial clinic visit prior to a formal pre-procedural evaluation. This nomogram can be readily converted to an easily accessible computer-based algorithm, which quickly provides information about estimated individual patient risk. However, additional interpretative safeguards should be included in such computer-based algorithm, stating that the nomogram still requires external validation. This would prevent discouraging complex patients and their providers from considering radioembolization.
We evaluated the value of a modified infusion technique for ${ }^{90} Y$ radioembolization [22]. ${ }^{9} \mathrm{Y}$ resin microspheres are the most commonly used radioembolization microspheres type for CRC liver metastases. Due to the high number of resin microspheres infused during the procedure, more uniform tumor coverage can be achieved compared to glass-based ${ }^{\circ} \mathrm{Y}$ microspheres. The main disadvantage of infusing high number of microspheres is the occurrence of stasis. Stasis has been reported in $>30 \%$ of infusions for the treatment of CRC liver metastases [21, 22]. It can lead to non-target radiation delivery, with consequent development of gastrointestinal ulceration as well as inadequate radiation exposure of the target tumor.

We proposed a modified ${ }^{\circ} \mathrm{Y}$ resin microspheres infusion technique, which includes administering undiluted contrast medium in both " $\mathrm{B}$ " and "D" infusion lines. It enables better real-time monitoring of administration of resin microspheres (compared to the standard sandwich technique) and better detection of flow reduction to minimize the risk of stasis. Our research demonstrated that the modified infusion technique is safe, with no evidence of radiation leaching or increased microspheres clotting. Even though the incidence of stasis was not significantly reduced, the modified infusion technique enabled shortening of resin microspheres infusion time, resulting in significant reduction of fluoroscopy radiation dose.

In CRC lung metastases over the last two decades, numerous studies had reported safety, good local disease control and lung parenchyma preservation following thermal ablation for unresectable oligometastatic patients [23-25]. Ablation of lung metastases has an important role for patients with limited disease, which can be ablated with sufficient minimal ablation margins $(\geq \mathbf{5 m m}$ ) as well as for those who present with residual or recurrent disease after having undergone other local treatments. Despite the relative lack of comparative studies of thermal lung ablation with other local treatments such as radiation treatment for metastatic disease, existing evidence shows that thermal ablation compares favorably with surgical resection. Unlike surgical resection or stereotactic beam radiotherapy, thermal ablation can be repeated with minimal if any impact on pulmonary function. This is of particular benefit in the setting of recurrent or residual disease in a population where future metastases and need for repeated local therapy is rather the rule than the exception.

Our literature review showed that local disease progression remains a limitation of ablative therapy. Across ablation modalities, tumor size is the primary determinant of treatment success as it dictates the ability for sufficient minimal ablation margin creation. Development of new tissue and corresponding surrogate image biomarkers 
for patients risk stratification and earlier detection of local tumor progression is needed. These developments along with evolution of technology may allow the application of local thermal ablation in a larger patient population.

There is limited clinical data on lung MW ablation especially for metastatic disease of CRC origin. MW ablation has at least theoretical potential to overcome known limitations of RF ablation. These include diminished thermal conductivity of aerated lung and "heat sink phenomenon" that can impact the ability to create large and uniform ablation zones. Thus higher rates of complete ablation and sustained tumor control could be expected when using MW ablation.

Currently there is a lack of homogeneous patient population research to see what MW ablation has to offer for the patients with CRC lung metastases. Our study is one of the most robust studies wherein. We showed that MW ablation for CRC lung metastases is safe and efficient. We identified factors related to local tumor control and showed that minimal ablation margin of $\geq 5 \mathrm{~mm}$ is critical for local tumor control following lung ablation, similarly to liver ablation. Pleural-based metastases and larger tumor size were associated with higher risk of local tumor progression. We also identified that known factors such as increased CEA levels and an increased prior number of chemotherapy regimens (likely reflecting more advanced disease stage) negatively impacted cancerspecific survival.

\section{FUTURE PERSPECTIVES}

To date the evidence regarding whether increased local tumor control after interventional treatment of metastatic disease translates into increased overall survival is lacking. As IO techniques strive to take additional roles in the management of metastatic CRC, substantial prospective trial-based evidence is warranted. Although prospective clinical trials comparing the efficacy of thermal ablation, image guided beam radiotherapy and surgery are challenging to set up, they could define the role of these therapies and improve patient selection for each treatment. It may be as important to design trials to assess the potential synergistic role of local therapies' in combination with systemic options in an effort to prolong patients' survival while maintaining the best possible quality of life.

With immunotherapy increasingly applied for stage IV disease, understanding the abscopal effect of IO treatments in patients treated with immunotherapy is of high importance. This includes investigation of its role in tumor priming, immunomodulation and selective immunotherapy delivery. Such research will expand the role of IO and local therapies in general in the patient population with diffuse metastatic disease that in the end have no local options.

\section{CONCLUSION}

Interventional oncology has an expanding role in the local treatment of the patients with oligometastatic CRC. Understanding the role of IO therapies in the multidisciplinary spectrum of local therapies is crucial. In this thesis, we identified factors that can help to improve local tumor control and reduce the incidence of complications following lung and liver thermal ablations as well as ${ }^{\circ} \mathrm{Y}$ radioembolization for patients with CRC liver metastases. We showed the importance of minimal ablation margin of at least $5 \mathrm{~mm}$ for good local tumor control following thermal ablation. We identified relevant factors associated with biliary complications following liver thermal ablation. Obtaining a $>10 \mathrm{~mm}$ minimal ablation margin enabled durable liver tumor eradication in patients without biliary complications risk. However for the high-risk patients with a history of HAI therapy a 6-10 mm minimal margin is ideal to balance between an acceptable risk of morbidity and good local control. We developed a prognostic nomogram aimed to improve the selection of patients undergoing ${ }^{9} \mathrm{Y}$ radiomebolization in the advanced, chemorefractory salvage setting. This nomogram may be useful during pre-treatment patient assessment to obtain a realistic estimation of patients' survival, but further external validation is warranted before it can be implemented in routine clinical practice. We evaluated a modified resin microspheres infusion technique during ${ }^{90} \mathrm{Y}$ radioembolization which was demonstrated to be safe and to reduce infusion time and fluoroscopy radiation dose. Radiation segmentectomy was demonstrated to be safe, enabled to achieve high tumor response and local tumor control rate for the patients with liver metastatic disease and limited treatment options. Larger prospective studies defining the role of radiation segmentectomy for metastatic CRC patients are required. 


\section{REFERENCES}

1. Nielsen, K. et al. Incidence and treatment of local site recurrences following RFA of colorectal liver metastases. World journal of surgery 37, 1340-1347, doi:10.1007/soo268-0131997-6 (2013).

2. Abitabile, P., Hartl, U., Lange, J. \& Maurer, C. A. Radiofrequency ablation permits an effective treatment for colorectal liver metastasis. European journal of surgical oncology : the journal of the European Society of Surgical Oncology and the British Association of Surgical Oncology 33, 67-71, doi:10.1016/j. ejso.2006.10.040 (2007).

3. Hammill, C. W. et al. Outcome after laparoscopic radiofrequency ablation of technically resectable colorectal liver metastases. Annals of surgical oncology $\mathbf{1 8}$ 1947-1954, doi:10.1245/s10434-010-1535-9 (2011).

4. Otto, G. et al. Radiofrequency ablation as first-line treatment in patients with early colorectal liver metastases amenable to surgery. Annals of surgery 251, 796-803, doi:10.1097/SLA.obo13e3181bcgfae (2010).

5. Puijk, R. S. et al. Colorectal liver metastases: surgery versus thermal ablation (COLLISION) - a phase III single-blind prospective randomized controlled trial. BMC cancer $\mathbf{1 8}$, 821, doi:10.1186/s12885-018-4716-8 (2018).

6. Wang, X. et al. Margin size is an independent predictor of local tumor progression after ablation of colon cancer liver metastases. Cardiovascular and interventional radiology $\mathbf{3} 6$ 166-175, doi:10.1007/soo270-012-0377-1 (2013).

7. Shady, W.et al. Percutaneous Radiofrequency Ablation of Colorectal Cancer Liver Metastases: Factors Affecting Outcomes-A 10- year Experience at a Single Center. Radiology 278, 601-611, doi:10.1148/radiol.2015142489 (2016)

8. Kurilova, I. et al. Microwave Ablation in the Management of Colorectal Cancer Pulmonary Metastases. Cardiovascular and interventional radiology 41, 1530-1544, doi:10.1007/soo270-018-2000-6 (2018).

9. Fernandez Moro, C., Bozoky, B. \& Gerling, M. Growth patterns of colorectal cancer liver metastases and their impact on prognosis: a systematic review. BMJ open gastroenterology 5, eooo217, doi:10.1136/bmjgast-2018-000217 (2018).

10. Oliveira, R. C., Alexandrino, H., Cipriano, M. A. \& Tralhao, J. G. Liver Metastases and Histological Growth Patterns: Biological Behavior and Potential Clinical Implications-Another Path to Individualized Medicine? Journal of oncology 2019, 6280347, doi:10.1155/2019/6280347 (2019).

11. Liu, C. H. et al. Radiofrequency ablation of hepatic tumours: effect of post-ablation margin on local tumour progression. European radiology 20, 877-885, doi:10.1007/ soo330-009-1610-4 (2010).

12. Kim, Y. S., Rhim, H., Cho, O. K., Koh, B. H. \& Kim, Y. Intrahepatic recurrence after percutaneous radiofrequency ablation of hepatocellular carcinoma: analysis of the pattern and risk factors. European journal of radiology 59, 432-441, doi:10.1016/j. ejrad.2006.03.007 (2006).

13. Kim, Y. S. et al. The minimal ablative margin of radiofrequency ablation of hepatocellular carcinoma $(>2$ and $<5 \mathrm{~cm}$ ) needed to prevent local tumor progression: 3D quantitative assessment using CT image fusion. AJR American journal of roentgenology 195, 758-765, doi:10.2214/ajr.09.2954 (2010)

14. Nakazawa, T. et al. Radiofrequency ablation of hepatocellular carcinoma: correlation between local tumor progression after ablation and ablative margin. AJR. American journal of roentgenology $\mathbf{1 8 8}, \quad 480-488$, doi:10.2214/ajr.05.2079 (2007)

15. Kim, S. H. et al. Changes in bile ducts after radiofrequency ablation of hepatocellular carcinoma: frequency and clinical significance. AJR. American journal of roentgenology 183, 1611-1617, doi:10.2214 ajr.183.6.01831611 (2004)

16. Ding, J. et al. Complications of thermal ablation of hepatic tumours: comparison of radiofrequency and microwave ablative techniques. Clinical radiology 68, 608-615, doi:10.1016/j.crad.2012.12.008 (2013)

17. Kim, K. R. \& Thomas, S. Complications of image-guided thermal ablation of liver and kidney neoplasms. Seminars in interventional radiology 31, 138-148, doi:10.1055/s-0034-1373789 (2014).

18. Livraghi, T. et al. Treatment of focal liver tumors with percutaneous radio-frequency ablation: complications encountered in a multicenter study. Radiology 226, 441-451, doi:10.1148/radiol.2262012198 (2003).

19. Fonseca, A. Z., Santin, S., Gomes, L. G., Waisberg,J.\& Ribeiro, M.A., Jr. Complications of radiofrequency ablation of hepatic tumors: Frequency and risk factors. World journal of hepatology 6, 107-113, doi:10.4254 wjh.v6.i3.107 (2014)

20. Sotirchos, V. S. et al. Colorectal Cancer Liver
Metastases: Biopsy of the Ablation Zone and Margins Can Be Used to Predict Oncologic Outcome. Radiology 280, 949-959, doi:10.1148| radiol.2016151005 (2016).

21. Kurilova, I. et al. Factors Affecting Oncologic Outcomes of goY Radioembolization of Heavily Pre-Treated Patients With Colon Cancer Liver Metastases. Clinical colorectal cancer 18, 8-18, doi:10.1016/j.clcc.2018.08.004 (2019)

22. Kurilova, I. et al. (90)Y Resin Microspheres Radioembolization for Colon Cancer Liver Metastases Using Full-Strength Contrast Material. Cardiovascular and interventional radiology 41, 1419-1427, doi:10.1007/s00270-0181985-1 (2018).

23. Mouli, S. K., Kurilova, I., Sofocleous, C. T. \& Lewandowski, R. J. The Role of Percutaneous Image-Guided Thermal Ablation for the Treatment of Pulmonary Malignancies. AJR. American journal of roentgenology 209, 740-751, doi:10.2214/ajr.17.18368 (2017)

24. de Baere, T. et al. Radiofrequency ablation is a valid treatment option for lung metastases: experience in 566 patients with 1037 metastases. Annals of oncology: official journal of the European Society for Medical Oncology 26, 987-991, doi:10.1093/annonc/mdv037 (2015).

25. Qi, H. \& Fan, W. Value of ablation therapy in the treatment of lung metastases. Thoracic cancer 9, 199-207, doi:10.1111/1759-7714.12567 (2018) 


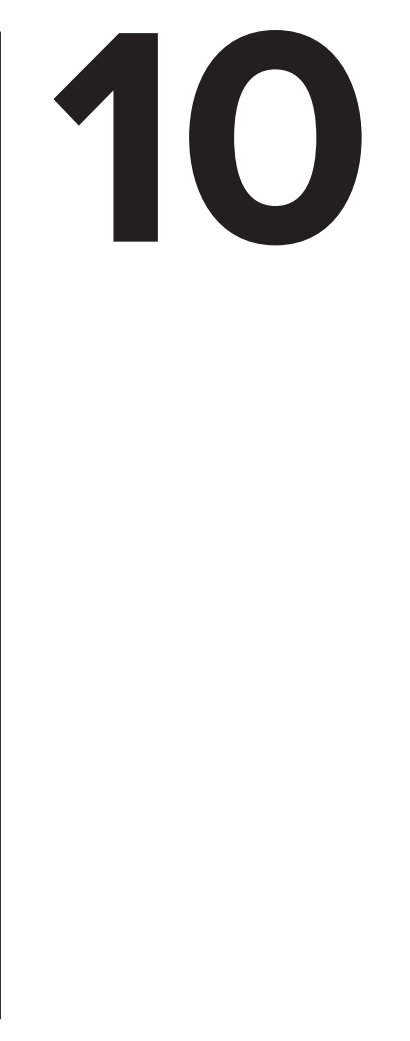


Chapter 2. Liver-directed and systemic therapies for colorectal cancer liver metastases

This literature review provided an overview on epidemiology, predisposing factors, pathology, diagnosis, approved/ recommended liver-directed and systemic therapies for metastatic CRC as well as it defined the role of interventional oncology in treatment of CRC liver metastases. The review also discussed standard of care as well as emerging interventional oncology procedures to treat metastatic CRC, new treatment modalities on the pipeline and key driving mutations which are important for better patient selection.

Chapter 3. Factors associated with local tumor control and complications after thermal ablation for colorectal cancer liver metastases: a 15-years retrospective cohort study

In our research we identified the factors associated with biliary complications - the most common type of major complications following liver thermal ablation in our study. We noted the strong association between post-ablation biliary complications with prior HAI therapy as well as with additional biliary complication predictors, such as pre-existing biliary dilatation, bevacizumab administration and minimum ablation margin size, which to the best of our knowledge, have not been described before. Our study provided recommendation to withhold bevacizumab within one month to thermal ablation, similarly to the precautions to the surgery [1]

Also, our study was one of the few studies documenting the importance of minimum ablation margin for a long-term tumor control by thermal ablation [2-9]. This study also supported the findings of our prior research group study that was limited on RFA, identifying minimal ablation margin size, increased tumor size and no history of prior hepatectomy as independent predictors of better LTPFS on multivariate analysis. We noted, that the best oncological outcomes were achieved for the patients treated with $>10 \mathrm{~mm}$ minimal ablation margins with no case of LTP recorded. In our study we analyzed the risks of creating a $>10 \mathrm{~mm}$ minimal ablation margin for two patient populations: patients with HAI history and HAI-naïve.

Chapter 4. Factors Affecting Oncologic Outcomes of ${ }^{90} Y$ Radioembolization of Heavily PreTreated Patients with Colon Cancer Liver Metastases

The developed nomogram included six easy-to-obtain pre-treatment parameters (sum of largest diameters of two largest liver lesions in intended to-treat-regio, number of extrahepatic disease sites, CEA level, alanine aminotransferase, tumor differentiation and albumin levels) and provided good prediction of patient survival postradioembolization. Baseline metabolic FDG-uptake parameter (SUVmax) was the single significant predictor of liver progression-free survival on multivariate analysis.
Chapter 5. ${ }^{9} Y$ Resin Microspheres Radioembolization for Colon Cancer Liver Metastases Using Full-Strength Contrast Material

Administration of ${ }^{\circ} \mathrm{Y}$ resin microspheres using undiluted contrast medium in both "B" and "D" infusion lines was demonstrated to be safe and effective, resulting in lower fluoroscopy radiation dose and shorter infusion time, without evidence of myelosuppression or increased stasis incidence.

Chapter 6. Radiation Segmentectomy for Hepatic Metastases with ${ }^{\circ} Y$ Glass-Based Microspheres: Technical Considerations, Correlations to Dosimetry and Preliminary Results

In this small retrospective study we described our initial experience with radiation segmentectomy in selected patients with limited metastatic liver disease that was not amenable to resection or percutaneous ablation. The study assessed the feasibility of treating up to three liver metastases using ${ }^{90} \mathrm{Y}$ radiation segmentectomy with local curative intent similar to ablative external radiation therapy or curative percutaneous ablation $[10,11]$. We demonstrated, that radiation segmentectomy of $\leq 3$ hepatic segments can safely provide good local tumor control in selected patients with limited metastatic liver disease and treatment options.

Chapter 7. Image-Guided Thermal Ablation for the Treatment of Pulmonary Malignancies This literature review provided an overview on a variety of image-guided thermal ablation techniques for patients with oligometastatic lung disease and non-small cell lung carcinoma.

Chapter 8. Microwave Ablation in the Management of Colorectal Cancer Pulmonary Metastases In this study we indicated, that the efficacy of MW ablation compared favorably to prior results of thermal ablation in terms of local tumor control, local progression-free and overall survivals [12-15]

Factors associated with local tumor progression-free survival and cancer-specific survival were identified and described. Firstly, we found strong association between tumor size and local tumor progression (LTP) and strong inverse association of LTP with minimal ablation margin, with all LTPs occurring in tumors $\geq 1 \mathrm{~cm}$, ablated with minimal margin of $<5 \mathrm{~mm}$. This observation supports the value of the minimal ablation margin as independent predictor of LTP as reported previously [2, 4, 16-18]. Also, this finding supports the fact, that tumor size remains a limiting factor for the widespread use of ablation regardless of energy used [19-22]. Pleural-based tumors had more than seven times the risk for LTP when compared to non-pleural-based metastases. Also, we found that increased CEA level and increased number of pre-MW ablation chemotherapy 
lines negatively impacted cancer-specific survival. Further validation of study results is certainly needed in larger studies with longer follow-up to better define the role of MW ablation in the management of CRC metastatic disease.

\section{REFERENCES}

1. Genetech, I. U.S. BL $125085 / 169$ Amendment: Bevacizumab-Genentech. San Francisco, CA, USA (2009)

2. Sotirchos, V. S. et al. Colorectal Cancer Liver Metastases: Biopsy of the Ablation Zone and Margins Can Be Used to Predict Oncologic Outcome. Radiology 280, 949-959, doi:10.1148 radiol.2016151005 (2016).

3. Wang, X. et al. Margin size is an independent predictor of local tumor progression after ablation of colon cancer liver metastases. Cardiovascular and interventional radiology 36, 166-175, doi:10.1007| soo270-012-0377-1 (2013).

4. Shady, W. et al. Percutaneous Radiofrequency Ablation of Colorectal Cancer Liver Metastases: Factors Affecting Outcomes-A 10-year Experience at a Single Center. Radiology 278, 601-611, doi:10.1148 radiol.2015142489 (2016).

5. Kaye, E. A. et al. Volumetric 3D assessment of ablation zones after thermal ablation of colorectal liver metastases to improve prediction of local tumor progression. European radiology, doi:10.1007| soo330-018-5809-o (2018).

6. Shady, W. et al. Percutaneous Microwave versus Radiofrequency Ablation of Colorectal Liver Metastases: Ablation with Clear Margins (Ao) Provides the Best Local Tumor Control. Journal of vascular and interventional radiology: JVIR 29, 268-275. e261, doi:10.1016|j.jvir.2017.08.021 (2018)

7. Cornelis, F. H. et al. Immediate Postablation (18) F-FDG Injection and Corresponding SUV Are Surrogate Biomarkers of Local Tumor Progression After Thermal Ablation of Colorectal Carcinoma Liver Metastases. Journal of nuclear medicine : official publication, Society of Nuclear Medicine 59, 1360-1365, doi:10.2967/jnumed.117.194506 (2018).
8. Odisio, B. C. et al. Local tumour progression after percutaneous ablation of colorectal liver metastases according to RAS mutation status. The British journal of surgery 104, 760-768, doi:10.1002/ bjs.1049o (2017).

9. Calandri, M. et al. Ablation of colorectal liver metastasis: Interaction of ablation margins and RAS mutation profiling on local tumour progression-free survival. European radiology 28, 2727-2734, doi:10.1007/s00330-017-5273-2 (2018).

10. Nair, V. J. \& Pantarotto, J. R. Treatment of metastatic liver tumors using stereotactic ablative radiotherapy. World journal of radiology 6, 18-25, doi:10.4329/wjr.v6.i2.18 (2014)

11. Van Tilborg, A. A. et al. Long-term results of radiofrequency ablation for unresectable colorectal liver metastases: a potentially curative intervention. The British journal of radiology 84, 556565, doi:10.1259/bjr/78268814 (2011).

12. Vogl, T. J. et al. Thermal Ablation of Colorectal Lung Metastases: Retrospective Comparison Among Laser-Induced Thermotherapy, Radiofrequency Ablation, and Microwave Ablation. AJR. American journal of roentgenology 207, 1340-1349, doi:10.2214 ajr.15.14401 (2016).

13. Wolf, F. J. et al. Microwave ablation of lung malignancies: effectiveness, $\mathrm{CT}$ findings, and safety in 50 patients. Radiology 247, 871-879, doi:10.1148| radiol.2473070996 (2008).

14. Lu, Q. et al. CT-guided percutaneous microwave ablation of pulmonary malignancies: Results in 69 cases. World J Surg Oncol 10, 80, doi:10.1186/1477-781910-80 (2012).

15. Wolf, F. J., Aswad, B., Ng, T. \& Dupuy, D. E. Intraoperative microwave ablation of pulmonary malignancies with tumor permittivity feedback 
control: ablation and resection study in 10 consecutive patients. Radiology 262, 355-360, doi:10.1148/radiol.11110015 (2012).

16. Yan, T. D. et al. Percutaneous radiofrequency ablation of pulmonary metastases from colorectal carcinoma: prognostic determinants for survival. Annals of surgical oncology 13, 1529-1537, doi:10.1245/ s10434-0o6-9101-1 (2006).

17. Mouli, S. K., Kurilova, I., Sofocleous, C. T. \& Lewandowski, R. J. The Role of Percutaneous ImageGuided Thermal Ablation for the Treatment of Pulmonary Malignancies. AJR. American journal of roentgenology 209, 740-751, doi:10.2214/ajr.17.18368 (2017).

18. Giraud, P. et al. Evaluation of microscopic tumor extension in non-small-cell lung cancer for threedimensional conformal radiotherapy planning. Int J Radiat Oncol Biol Phys 48, 1015-1024 (2000).

19. Vogl, T. J et al. Microwave ablation therapy: clinical utility in treatment of pulmonary metastases. Radiology 261, 643-651, doi:10.1148/radiol.11101643 (2011).

20. Steinke, K. et al. Percutaneous imaging-guided radiofrequency ablation in patients with colorectal pulmonary metastases: 1-year follow-up. Annals of surgical oncology 11, 207-212 (2004).

21. Akeboshi, M. et al. Percutaneous radiofrequency ablation of lung neoplasms: initial therapeutic response. Journal of vascular and interventional radiology: JVIR 15, 463-470 (2004)

22. Simon, T. G., Beland, M. D., Machan, J. T., Dipetrillo, T. \& Dupuy, D. E. Charlson Comorbidity Index predicts patient outcome, in cases of inoperable non-small cell lung cancer treated with radiofrequency ablation. European journal of radiology 81, 4167-4172, doi:10.1016/j.ejrad.2012.06.007 (2012). 
Colorectal cancer (CRC) is one of the deadliest cancers, with metastatic disease being the most common cause of death in these patients [1]. Netherlands is among top ten countries in the world with the highest CRC prevalence [2]. On a daily basis around 37 patients receive CRC diagnosis and 14 patients die from CRC in the Netherlands [3]. The incidence of CRC is expected to rise even further because of the implementation of nationwide screening program [4] growing incidence in rectal cancer among young patients [5-7] and ageing population.

To date, the awareness of general population, patients and referring physicians about the field of interventional oncology and what it has to offer for the patients with CRC is low [8]. Thus active participation of those in the field is crucial to help to educate the public about many potential benefits interventional radiology may offer.

\section{RELEVANCE}

With this thesis, we aimed to provide an overview about the expanding role of interventional oncology in the management of the patients with metastatic CRC. We provided the data on oncological outcomes following the most common as well as emerging interventional oncology procedures. These procedures included thermal ablation (in the liver and lungs) and ${ }^{90} \mathrm{Y}$ radioembolization approaches. Notably, we analyzed predictive and prognostic factors for local tumor control, safety and survival following these therapies.

The research presented in this thesis was performed within the collaborative research project between the Netherlands Cancer Institute and Memorial Sloan Kettering Cancer Center (New York, USA). Memorial Sloan Kettering Cancer Center performs one of the largest numbers of interventional oncology procedures in the world, which enables to conduct large scale laboratory and clinical research. This collaboration was formed in order to profit from the expertise which is present there in the area of most modern minimally invasive approaches for the treatment of metastatic colorectal cancer and transfer this knowledge to the Netherlands.

\section{TARGET POPULATION}

This thesis has a wide target population. It includes interventional radiologists who are utilizing these procedures in their daily practice and who could benefit from applying the techniques described here to achieve better tumor control, increase procedure safety and improve patient selection. It is also very important to increase awareness about interventional radiology procedures among medical oncologists and other medical specialists involved in the management of the patients with metastatic colorectal cancer so that the patients could be more often referred to interventional radiology. Minimally invasive procedures have many advantages over traditional treatments, including lower morbidity and mortality, also sometimes representing the patients an only viable treatment option.

Colorectal cancer patients with metastatic disease could benefit from this thesis in multiple ways. Currently there is a lack of awareness among the patients about interventional radiology [8]. This may result in patients favoring traditional therapies over unfamiliar minimally invasive procedures despite lower complication rates, faster recovery time and a better quality of life. There is evidence that the patients referred for an interventional radiology procedure largely do not know very much if anything about the field, even if they had undergone an interventional radiology procedure in the past [8]. Efforts to increase public awareness about this field could improve patient access to these procedures and empower patients with the information they need to make the best healthcare decisions themselves.

The patients with other types of oligometastatic cancer also could benefit from our research as the interventional radiology procedures we described are used for multiple types of oligometastatic cancer.

Researchers, conducting clinical and fundamental research in interventional radiology, colorectal cancer as well as in other local therapies could also find this thesis useful. Multidisciplinarity and combinational treatments are very important to maximize treatment outcomes in the patients with metastatic CRC.

\section{INNOVATION AND FUTURE}

The results of this thesis demonstrated that it is possible to achieve better local tumor control with thermal ablation while creating a sufficient minimal ablation margin [9]. We also identified complications risk factors following thermal ablation and provided recommendation on how to approach high-risk patients [10]. In terms of ${ }^{\circ} \mathrm{Y}$ radioembolization approaches, we developed a prognostic tool aimed at improving patient selection [11] as well as proposed a modified ${ }^{\circ} \mathrm{Y}$ resin microspheres infusion 
technique to improve the procedure safety [12]. We also filled in the knowledge gap on radiation segmentectomy for the patients with liver metastatic disease and limited treatment options, demonstrating that it has a potential to safely achieve high tumor response and local tumor control rate.

Currently minimal ablation margin assessment is not performed universally and uniformly among interventional radiologists immediately post-procedure. Ablative dose radioembolization approaches, such as radiation segmentectomy are emerging, but are also not yet utilized in multiple centers due to the limited evidence available to date. There is a lack of prognostic tools for physicians to use during pre-procedure patient visit to help decision-making. Our research tried to address all these limitations; however larger prospective trials, aiming at externally validating our retrospective study results are warranted.

In addition, despite expanding evidence regarding the value of interventional oncology procedures to provide local disease control, data on the effect of these procedures on patients' overall survival is lacking. Prospective studies comparing different locoregional modalities to surgery are also limited. Future research should also focus on the identification of the patients with curable oligometastatic CRC disease, systemic effects of interventional radiology procedures and immunomodulation. As interventional oncology techniques strive to take additional roles in the management of the patients with metastatic CRC, progress will ultimately depend on the ability to produce substantial prospective trial-based evidence.

\section{REFERENCES}

1. Araghi, M. et al. Global trends in colorectal cancer mortality: projections to the year 2035. International journal of cancer 144, 2992-3000, doi:10.1002/ijc.32055 (2019).

2. Bray, F. et al. Global cancer statistics 2018: GLOBOCAN estimates of incidence and mortality worldwide for 36 cancers in 185 countries. CA: a cancer journal for clinicians 68, 394-424, doi:10.3322 caac.21492 (2018).

3. KWF Kanker Bestrijding. Darmkanker, <https://www. kwf.nl/kanker/darmkanker> (2019)

4 Cress, R. D., Morris, C., Ellison, G. L. \& Goodman, M. T. Secular changes in colorectal cancer incidence by subsite, stage at diagnosis, and race/ethnicity, 1992-2001. Cancer 107, 1142-1152, doi:10.1002/cncr.22011 (2006)

5. Tricoli, J.V.et al. Biologic and clinical characteristics of adolescent and young adult cancers: Acute lymphoblastic leukemia, colorectal cancer, breast cancer, melanoma, and sarcoma. Cancer 122, 10171028, doi:10.1002/cncr.29871 (2016)

6. Siegel, R. L., Jemal, A. \& Ward, E. M. Increase in incidence of colorectal cancer among young men and women in the United States. Cancer epidemiology, biomarkers \& prevention: a publication of the American Association for Cancer Research, cosponsored by the American Society of Preventive Oncology 18, 1695-1698, doi:10.1158/1055-9965.Epi-og0186 (2009).

7. O'Connell, J. B. et al. Rates of colon and rectal cancers are increasing in young adults. The American surgeon 69, 866-872 (2003).

8. Baerlocher, M. O. et al. Awareness of interventional adiology among patients referred to the interventional radiology department: a survey of patients in a large Canadian community hospital.
Journal of vascular and interventional radiology : JVIR 18, 633-637, doi:10.1016/j.jvir.2007.02.034 (2007)

9. Kurilova, I. et al Microwave Ablation in the Management of Colorectal Cancer Pulmonary Metastases. Cardiovascular and interventional radiology 41, 1530-1544, doi:10.1007/s0o270-018-20oo$6(2018)$.

10. I. Kurilova, E. K., F. Boas, H. Yarmohammadi, M. Gonen, E. Petre, N. Kemeny, S. Solomon, R. BeetsTan, C. Sofocleous. Review of complications following thermal ablation of colorectal cancer liver metastases. JVIR, https:|/doi.org/10.1016/j. jvir.2018.01.377 (2018).

11. Kurilova, I. et al. Factors Affecting Oncologic Outcomes of goY Radioembolization of Heavily Pre-Treated Patients With Colon Cancer Liver Metastases. Clinical colorectal cancer 18, 8-18, doi:10.1016/j.clcc.2018.08.004 (2019).

12. Kurilova, I. et al. (90)Y Resin Microspheres Radioembolization for Colon Cancer Liver Metastases Using Full-Strength Contrast Material. Cardiovascular and interventional radiology 41, 14191427, doi:10.1007/soo270-018-1985-1 (2018) 


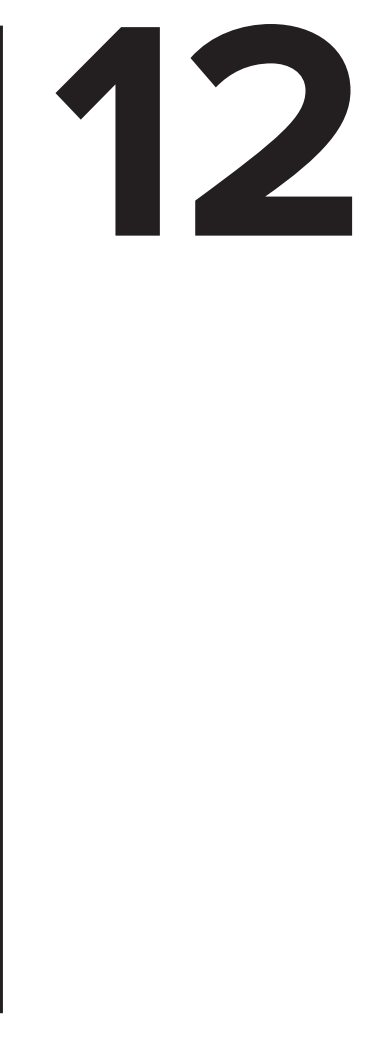


1. Kurilova, I., Beets-Tan, R. G. H., Flynn, J., Gonen, M., Ulaner, G., Petre, E. N., Boas F.E., Ziv E.,Yarmohammadi H., Klompenhouwer E.G., Cercek A., Kemeny N.A., Sofocleous, C. T. (2018). Factors Affecting Oncologic Outcomes of ${ }^{90} \mathrm{Y}$ Radioembolization of Heavily Pre-Treated Patients With Colon Cancer Liver Metastases. Clin Colorectal Cancer. doi:10.1016/j.clcc.2018.08.004

2. Kurilova, I.*, Gonzalez-Aguirre A.*, Beets-Tan R. G.H., Erinjeri J., Petre E. N., Gonen M., Bains M., Kemeny N. E., Solomon S. B., Sofocleous C. T. (2018). Microwave Ablation in the Management of Colorectal Cancer Pulmonary Metastases. Cardiovasc Intervent Radiol. http://dx.doi.org/10.1007/soo270-018-200o-6.

3. Kurilova, I., Beets-Tan R. G. H., Ulaner G. A., Boas F. E., Petre E. N., Yarmohammadi H., Ziv E., Deipolyi A. R., Brody L. A., Gonen M., Sofocleous C. T. (2018). (90)Y Resin Microspheres Radioembolization for Colon Cancer Liver Metastases Using FullStrength Contrast Material. Cardiovasc Intervent Radiol. http://dx.doi.org/10.1007/ soo270-018-1985-1.

4. Kemeny, N.*, Kurilova, I.*, Li, J.*, Camacho, J. C. \& Sofocleous, C. T. Liver-Directed and Systemic Therapies for Colorectal Cancer Liver Metastases. Cardiovascular and interventional radiology, doi:10.1007/soo270-019-02284-9 (2019).

5. Mouli S.K., Kurilova I., Sofocleous C.T., Lewandowski R.J. (2017). The Role of Percutaneous Image-Guided Thermal Ablation for the Treatment of Pulmonary Malignancies. AJR Am J Roentgenol. 209(4):740-751. doi: 10.2214/AJR.17.18368. Epub 2017 Jun 28. Review. PMID: 28657845

6. Kurilova I., Bendet A., Petre N.E., Boas F.E., Kaye E., Gonen M., Covey A., Brody L.A., Brown K.T., Kemeny N.E., Yarmohammadi H., Ziv E., MD, D’ Angelica M.I, Kingham P.T., Solomon S.B., Beets-Tan R.G.H., Sofocleous C.T. Factors associated with local tumor control and complications after thermal ablation for colorectal cancer liver metastases: a 15-years retrospective cohort study. Submitted to Clinical Colorectal Cancer.

7. Kurilova I., Bendet A., Fung E.K., Humm J.L., Do R.K.G., Boas F.E., Yarmohammadi H., Ziv E., Deipolyi A., Petre E.N., D’ Angelica M.I., Crane C.H., Kingham P.T, Cercek A., Beets-Tan R.G.H., Sofocleous C.T. Radiation Segmentectomy for Hepatic Metastases with ${ }^{\circ} \mathrm{Y}$ Glass-Based Microspheres: Technical Considerations, Correlations to Dosimetry and Preliminary Results. Submitted to American Journal of Roentgenology ${ }^{*}$ Shared first authorship 
I would like to extend my sincerest thanks to my promotor, prof. dr. Regina Beets-Tan for letting me join her research team and giving me the unique research fellowship opportunity at Memorial Sloan Kettering Cancer Center (MSKCC). This fellowship was an extremely valuable chance to learn from the experts in the area of interventional radiology (IR) from the very beginning of my research path. Thank you very much for your kindness, support and encouragement during my fellowship overseas as well as back in the Netherlands Cancer Institute (NKI-AVL).

I would also like to sincerely thank my co-promotor, prof. Dr. Constantinos T. Sofocleous for directly supervising me. Thank you very much for your attention and quick response to all my questions on a daily basis during the entire PhD study time, even from abroad. Thank you for putting the best effort to support me and give important tips for the presentations at different IR congresses, which I attended for the first time in my life. Working with Dr. Sofocleous helped me to considerably improve my scientific writing style, which became more structured and concise. I also learned not to relax after achievement and always work further. It is always a pleasure to meet Dr. Sofocleous at conferences since my fellowship in MSKCC.

Very special thanks for the senior research scientist of Department of Radiology at MSKCC, Dr. Elena Nadia Petre. Nadia is a person without whom I cannot imagine succeeding in this research. Nadia was the first person I would ask for help or advice, both from the scientific as well as the personal perspective. I am very happy that I could work right next to Nadia for 1.5 years and ask questions regarding statistics and the finer points of IR research on a daily basis. It was very nice of Nadia to always invite me over for nice lunch, help fix the laptop or share the heater when it would be very cold in the office. Thank you very much.

I would like to thank the interventional radiology team of MSKCC and all manuscript coauthors for their advice and contribution, including prof. Mithat Gonen for his friendly consultations for every paper. Also, special thanks to Dr. Govindarajan Srimathveeravalli, for his very valuable research insights, important advice and for the humor.

I would like to thank Dr. Elisabeth Klompenhouwer for being my NKI-AVL research supervisor and for giving valuable advice during my fellowship. Also, I would like to thank Dr. Fernando Gomez-Munoz for giving me the opportunity and support to work on multiple interesting IR research projects at the NKI-AVL. It was very exciting and has helped me acquire valuable knowledge in this area.
I would like to thank my fellow colleagues PhD students from the Radiology Department of the NKI-AVL, who surrounded me in the last year of my research. Thank you for your nice company, advice, help, and support and for making every day in the office a happy day. I couldn't ask for better colleagues.

I would like to thank my family for their constant support and encouragement. I would like to thank my friends who provided me with a great support and became my second family while I was in New York and in the Netherlands. I highly appreciate it and I could not accomplish this without you 
Ieva Kurilova was born on $6^{\text {th }}$ December 1991 in Vilnius, Lithuania. In 2010-2016 she studied Medicine in the Vilnius University, where besides medical studies she was conducting the clinical research in the areas of lung cancer and gastric neuroendocrine tumors. After graduating medical school Ieva became research fellow in the Radiology department of Netherlands Cancer Institute-Antoni van Leeuwenhoek Hospital, working on imaging in immunotherapy.

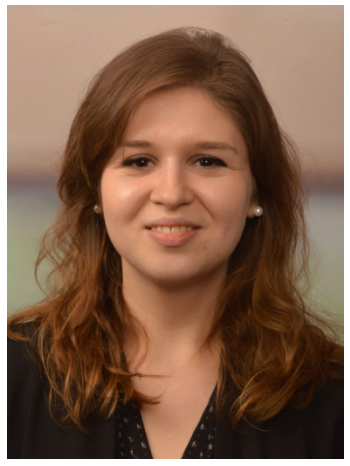

In December 2016 Ieva became a PhD student in the Radiology Department of NKIAVL, working under the supervision of Prof. Regina G.H. Beets-Tan. Majority of the PhD research she conducted within the collaborative research project in Memorial Sloan Kettering Cancer Center, New York, USA, where she worked under supervision of Prof. Dr. Constantinos T. Sofocleous.

Ieva has presented her PhD research results in the largest Radiology/ Interventional Radiology congresses, including Radiology Society of Northern America (RSNA), World Congress of Interventional Oncology (WCIO), Society of Interventional Radiology (SIR) and Cardiovascular and Interventional Radiology Society of Europe (CIRSE). Ieva has also been a European Research Seed Grant winner (2020), WCIO Fellow and Residentin-training Scholarship winner (2018) as well as Rolf W. Günter Foundation Young Radiologist's Travel Grant winner (2018). Ieva is also a member of good standing of ESR, CIRSE, ESOI and ARRS radiological societies. She is also a reviewer for the journals in the area of interventional radiology, such as Cardiovascular and Interventional Radiology journal (CVIR) and Journal of Vascular and Interventional Radiology (JVIR).

Currently, besides conducting clinical research, Ieva is actively assisting in setting up prospective clinical trials in the area of metastatic colorectal cancer treatment in interventional radiology setting, development of novel imaging biomarkers as well as student supervision in the Netherlands Cancer Institute. 
